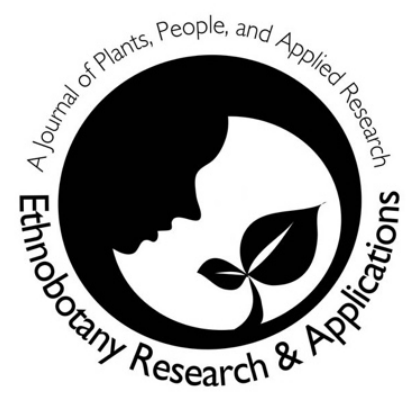

\title{
"How I became an ethnobotanist" an interview with ERA Associate Editor Dr. Narel Paniagua Zambrana
}

Maroof Ali Turi

\author{
Correspondence \\ Maroof Ali Turi \\ College of Life Science, Anhui Normal University, Wuhu 241000, China \\ *Corresponding Author: marufturi059@gmail.com; maturi@bs.qau.edu.pk \\ Ethnobotany Research and Applications 23:1 (2022)
}

\section{Notes on Ethnobotany}

\section{Abstract}

An interview with Narel Paniagua-Zambrana, Senior Scientist of the Department of Ethnobotany at the Institute of Botany, Ilia State University, Georgia, Associated Researcher of Herbario Nacional de Bolivia and co-director of Saving Knowledge. Her work focuses on ethnobotanical research and the preservation of traditional knowledge, in the Andes, the Caucasus, and the Himalayas.

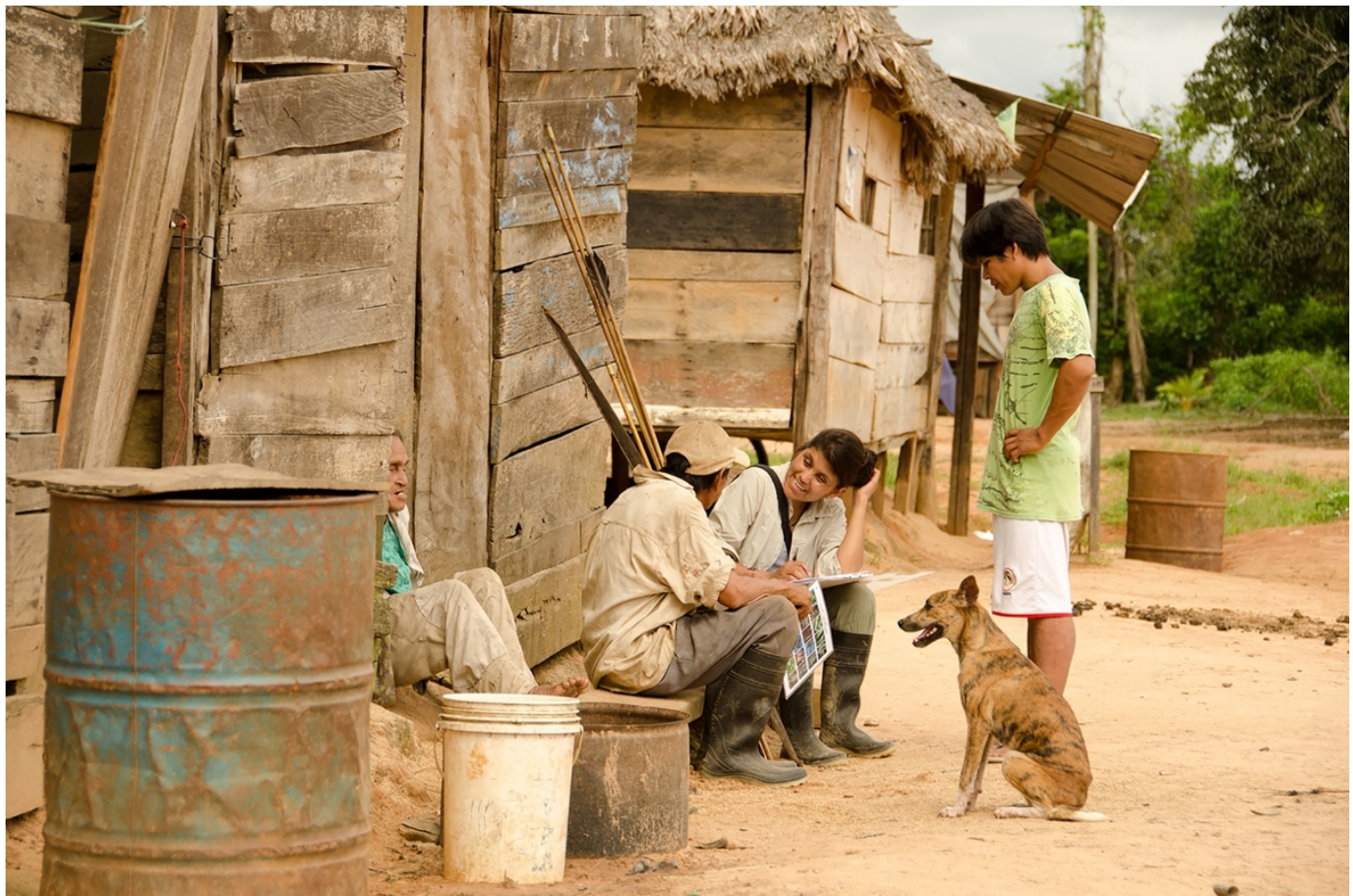

Narel Paniagua-Zambrana interviewing Ese Eja indigenous people in the community of Palma Real, Tambopata, Peru (Photo RW Bussmann). 


\section{Do you consider yourself primarily an ethnobotanist or do you have another way of seeing yourself?}

I am an ethnobotanist, and I study the complex relationship that people establish with plants through their use. This implies aspects related to their culture, their history, their socioeconomic context, and the environment in which they develop. I started studying ecology, seeking to understand the effect of the use of plants on ecosystems, and very quickly I understood that without incorporating people, their vision, knowledge, and perceptions, we could never conserve the ecosystems and plants that we wanted to protect. We now collaborate with local communities to protect and preserve their knowledge as the most important tool in conservation.

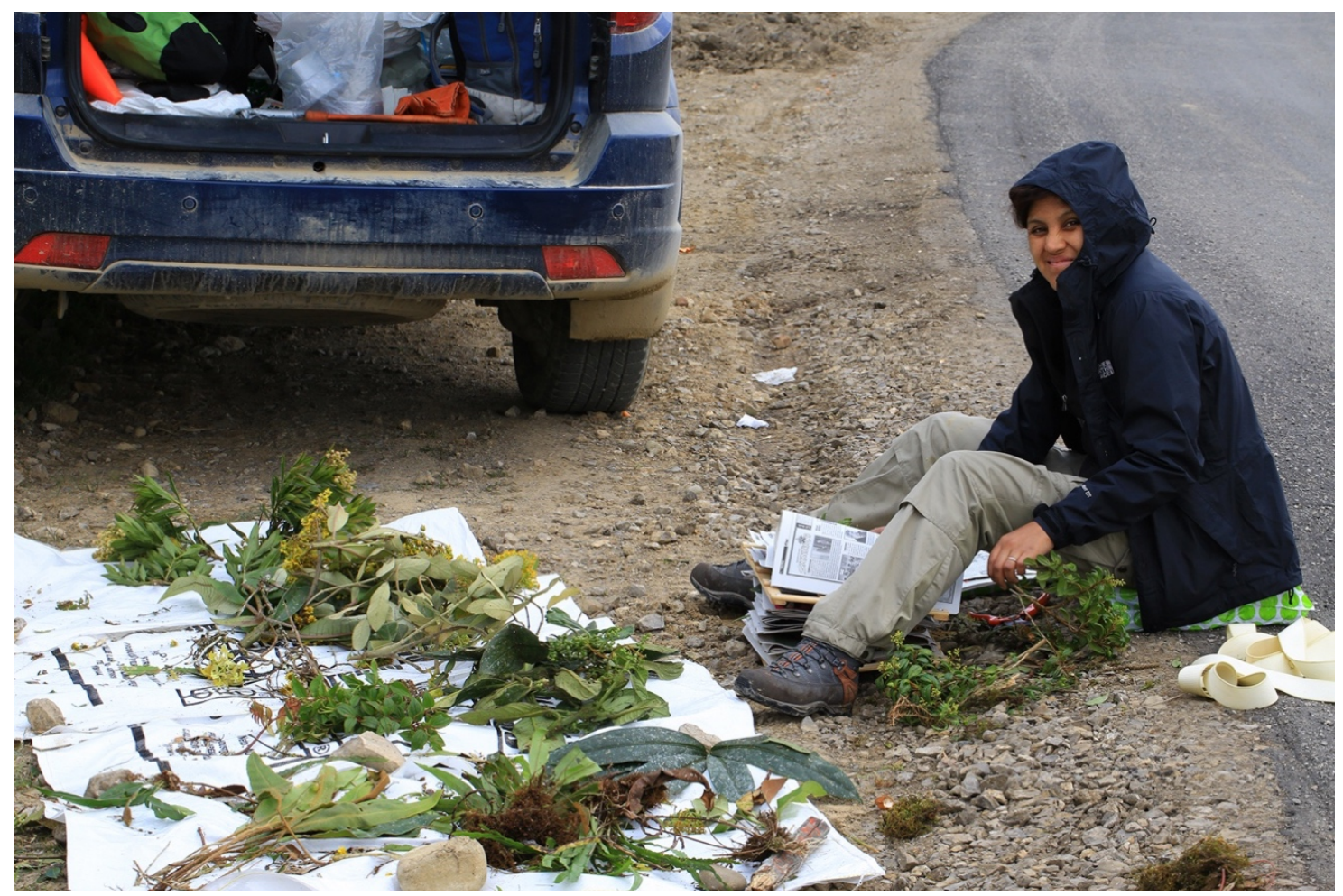

Pressing the collection of the day, one of the most common activities in botany and ethnobotany. Rio Marañon, Peru 2013 (Photo RW Bussmann).

\section{Describe your work/what you see as your mission}

My motivation / mission has changed a lot over the years that I have been collaborating with indigenous and local communities. It started with a fascination for the way people use plants, and now it has morphed into a deep respect for the way they "relate" to plants. After more than 15 years working on this issue, I must say that the word "use" does not fit at all for the "relationship" that people establish with plants that allows them to take advantage of their benefits. It is a relationship that involves social, cultural, historical, economic, and environmental aspects, and it is as complex as it is fascinating.

And precisely to contribute to understanding this complex relationship, our research consists of documenting this knowledge and correctly identifying the plants to which people attribute a use. In this way we have the information on use, local names and local classification provided by members of the community, and we also have a scientific collection of plants that allows us to know exactly what species we are talking about. These collections are deposited in local (national) herbaria, and in this way, they also serve to protect the Natural Heritage in their own countries.

In addition, the results of all our research have been returned to the local populations in formats and languages chosen by them (Balslev et al., 2012; Paniagua-Zambrana et al., 2011, 2012a - e, 2017c; Paniagua-Zambrana \& Bussmann 2018; Vega et al., 2014), and in many cases this material has been incorporated into educational materials in local schools or as support material for declarations of national heritage. In this way we support the processes of transmission of knowledge between generations, which in many communities is at risk of being lost due to so many social, environmental, and cultural changes to which they have been subjected. Seeing the reaction of people, old and young, when they hold the books is a huge motivation. 
An important aspect has been to recognize local participants as the main authors in all our publications, including scientific articles published in International Journals (Paniagua-Zambrana et al., 2017a, 2018a - d). This form of publication guarantees the intellectual property of this knowledge for the owners of the knowledge because this information is in the public domain and cannot be patented. And it also puts the decision of how to use it in your hands.

All our investigations are carried out with a Prior Informed Consent, which guarantees that they have the approval and permission of the communities where we work. The methods we apply are discussed and modified according to their requirements (Paniagua-Zambrana \& Bussmann, 2021). In this way we ensure that the information we generate responds to a local need and that in the future it can be used as a tool that helps them make better decisions, use, management and / or conservation of their resources or the knowledge associated with them. Certainly, you cannot protect or conserve what you do not know.

Another important aspect in our investigations has been the repatriation of ethnobotanical information to the communities (Paniagua-Zambrana et al., 2014a, 2018e), in order to allow them to have all the information that was part of previous investigations and that previously was never returned to them

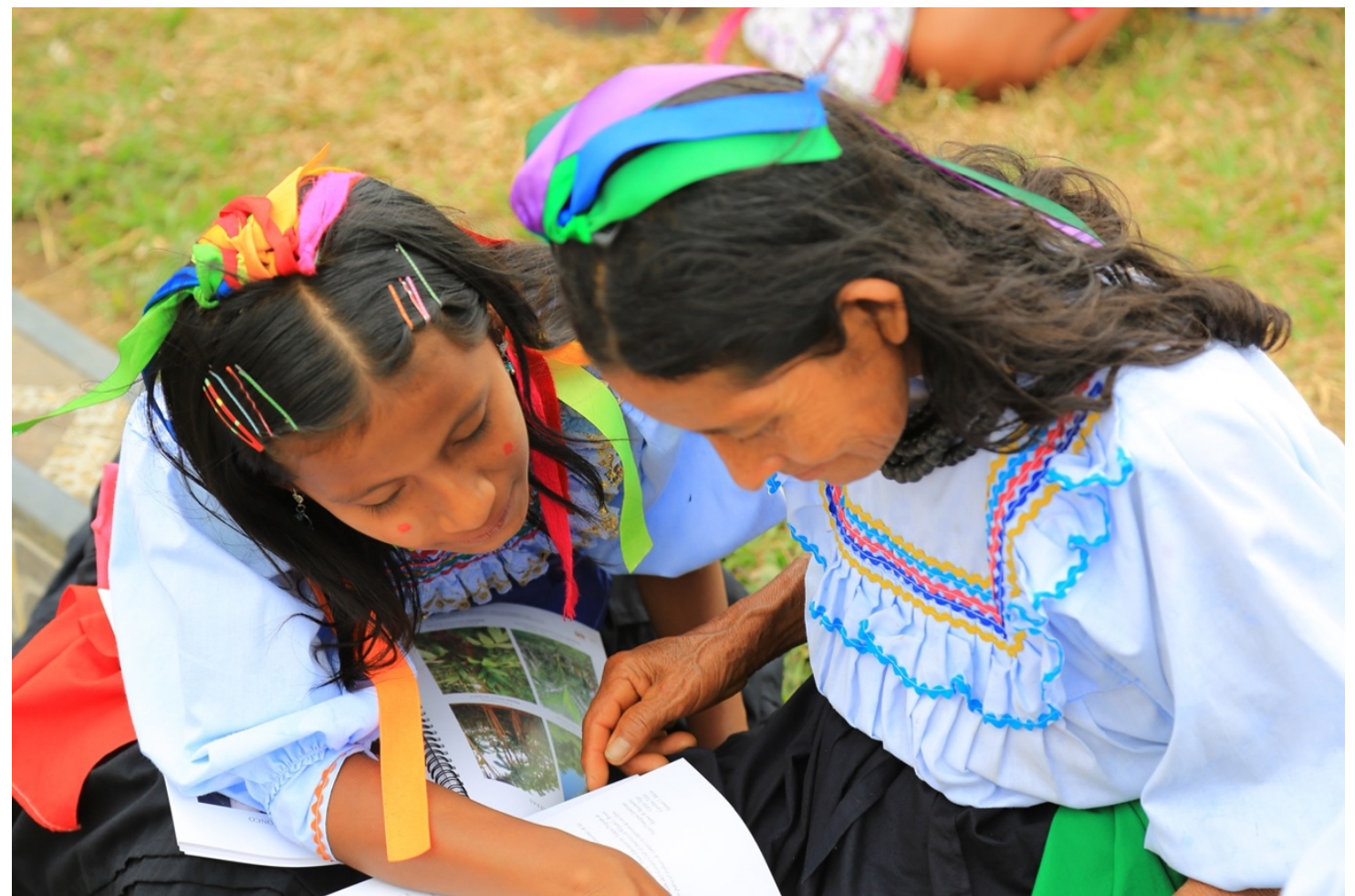

Grandmother and granddaughter of the Lamas indigenous group in Peru reading the books that were produced as result of our research, generating a processes of knowledge transmission (Photo RW Bussmann).

\section{Did you ever think you would be an ethno/botanist growing up? [Or work with plants?]. What did you want to be when you were a child?}

I grew up and studied in the mining camps in Bolivia, in small towns nestled in the mountain ecosystems above $4000 \mathrm{~m}$ of altitude. As a child, I would never have imagined being an ethnobotanist, not even a botanist. For me, plants were just another "toy", or part of an environment that we enjoyed.

My oldest and closest memory to people and the use of plants are the weekend "peasant fairs". The peasants came from the towns or farms near the camps to exchange or sell their agricultural products. These were days that I was looking forward to a lot during the week, because there was always the possibility of trying or seeing something new. These experiences were the ones that initially made me to decide studying agronomy. After a quick passage (one class only) through agronomy, I discovered biology and with-it ethnobotany. 


\section{What/where did you study? Do you think this influences you in any particular way? If you were not an ethnobotanist, what would you be?}

I studied Biology at the Universidad Mayor de San Andrés in La Paz, Bolivia. After spending the first years as a volunteer in various departments, I decided to study Botany because in 1993 was accepted into a Rapid Biology Assessments course organized by Conservation International, as member of the botanist team. There, under the guidance of one of the world's leading specialists in Tropical Flora, Dr. Robin Foster of the Field Museum in Chicago, I began my passion for plants. Until then I had never seen a tropical forest. Learning to know a forest as Dr. Foster showed us, was the best gift I could have in my first steps as a botanist. His incredible knowledge, his humility, and his patience were a very important example for me as a person. As a result of this trip, I decided to choose a species of palm tree widely used in the Amazon, Attalea princeps Mart, fort my undergraduate thesis, for which I studied the impact of the use of fruits and leaves on the natural populations of this species, with a primarily ecological and management approach (Paniagua-Zambrana \& Moraes, 2009). It was my first approach to ethnobotany.

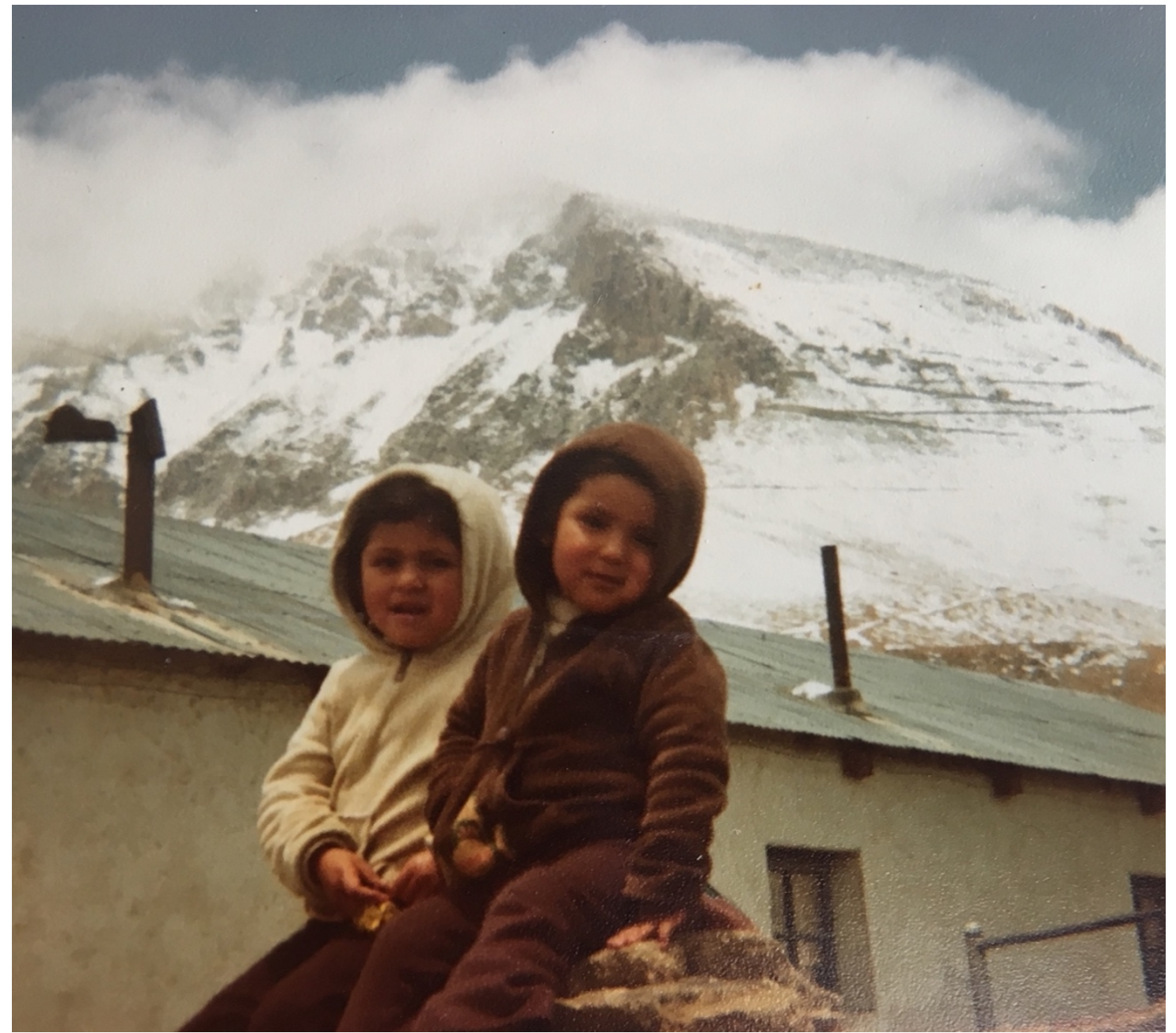

Narel Paniagua-Zambrana with her brother in the Chorolque mining camp at an altitude of 4850 m, 1976. (Family Photo)

My passion for ethnobiology was consolidated when I again chose palm trees as my study group in my post graduate studies. In 2005 I obtained my Masters' degree at the University of Aarhus in Denmark, and I had the opportunity to develop my thesis comparing the factors that influence the knowledge and use of palm trees in two regions, one in Peru and the other in Bolivia (Paniagua-Zambrana et al., 2007). This opportunity allowed me to meet and learn from different specialists in palm ecology and taxonomy and it was a very important opportunity for the development of my career. However, my most important experience and the one that defined me as ethnobotanical, was the development of my doctoral thesis. In 2010 I began my doctoral studies in Biology at the Autonomous University of Madrid, Spain, as part of a regional project that sought to understand the impact of the use of palm trees in tropical forests. My research was part of the component that studied the use of palm trees, specifically seeking to understand what the socioeconomic factors that determined the knowledge of people in the north-west region of the Amazon 
were (Bussmann \& Paniagua-Zambrana, 2013; Paniagua-Zambrana et al., 2014b, 2015, 2016, 2017b). The research had a fairly ambitious methodological approach (Paniagua-Zambrana et al., 2010). We had to cover all forests below $1000 \mathrm{~m}$ in the Amazon, the Andes, and the Chocó, conducting interviews in four countries (Ecuador, Colombia, Peru, and Bolivia), including information on the distribution and impact of the socioeconomic conditions that we were interested in evaluating. On over 18 months in the field, we conducted approximately 2300 interviews in 68 communities and 38 different indigenous groups, I believe that I will never in my life speak with so many different people in a single year.

But, beyond the "impressive" data we obtained, this experience allowed me to have a much more realistic perception of the way in which people in the communities understand and define words like "traditional knowledge" and "use". It helped me to better understand the close relationship that they establish with the resources they use, and without a doubt this was the most important contribution to the future approach of our new research projects.

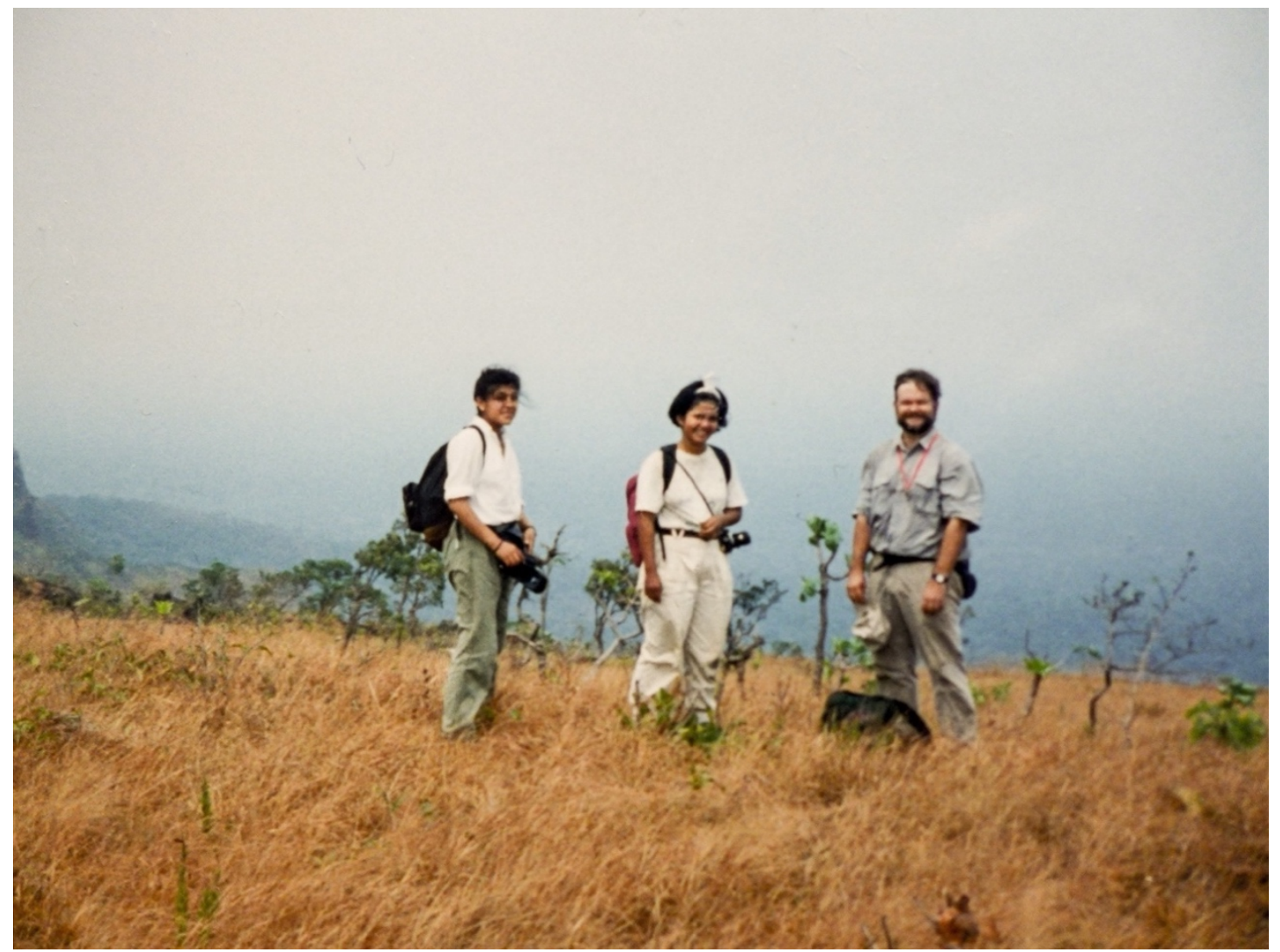

Together with Dr. Robin Foster and Dra. Marielos Peña in the Caparuch Mountains (Noel Kempff Mercado National Park, Bolivia), during the Rapid Biological Assessments course, 1993 (Photo NY Paniagua Zambrana)

\section{What got you interested in ethnobotany?}

My interest in studying the use of plants began with my undergraduate thesis where I studied the impact of the use of fruits and leaves on the natural populations of Attalea princeps Mart, A species of palm tree widely used in the Amazon, with a focus mainly ecological and management (Paniagua-Zambrana \& Moraes, 2009). Initially I underestimated the importance of people's knowledge in managing the species. It was a somewhat empirical ethnobotanical work, guided by the needs that appeared with the population data, but very important for the formulation of a management plan for the species. This was my first approach to ethnobotany.

But my interest was reinforced with an expedition organized by Conservation International in 1997 where I was invited as part of the team of botanists, to inventory the biodiversity of the Madidi National Park and Natural Area of Integrated Management, when it still did not have this category and its incredible biodiversity was hardly known. During that expedition we met an indigenous couple of the Tacana ethnic group, who for a whole day showed us plants and talked about the use that their people made of plants. A few years later I received my first research grant to develop my first ethnobotanical study in San José de Uchupiamonas, the Tacana community where this couple lived. Together with them and all the community members as authors, I wrote my first book, with which I returned 
traditional knowledge to each of the families in this indigenous community (Paniagua-Zambrana \& Comunidad San José de Uchupiamonas, 2001). The limitations of financial resources did not allow us to produce a book with the quality that we would have liked, they were simply ringed photocopies, but after more than 20 years a colleague who visited San José told me, that several members of the community still kept the book and used it in their ecotourism activities. And now, after many years researching under an academic system that only recognizes the Impact Factor of a journal as the impact of your publications, I can say that for me this is the true impact of our research, and it is what we try to show.

One reason to emphasize the devolution of information to the original knowledge holders is related to the importance of transmitting this knowledge to the younger generations. In most communities, knowledge transmission is oral and through direct practice. However, for different reasons, including economic, social, and even political, these processes have been changing and have even been lost. For example, the loss of the language is one reason why a lot of knowledge can no longer be transmitted, the new generations no longer speak the local language, so it is important to find a way to transmit this knowledge considering all these changes. Here enters the importance of documenting this knowledge and putting it in their hands in a language they understand and use. And many times, seeing how rich their knowledge is, the next step for young people is to learn their local language. Our research seeks to strengthen these transmission processes in a globalized and constantly changing world, understanding this important aspect we will be able to better focus our efforts so that these important processes still exist.

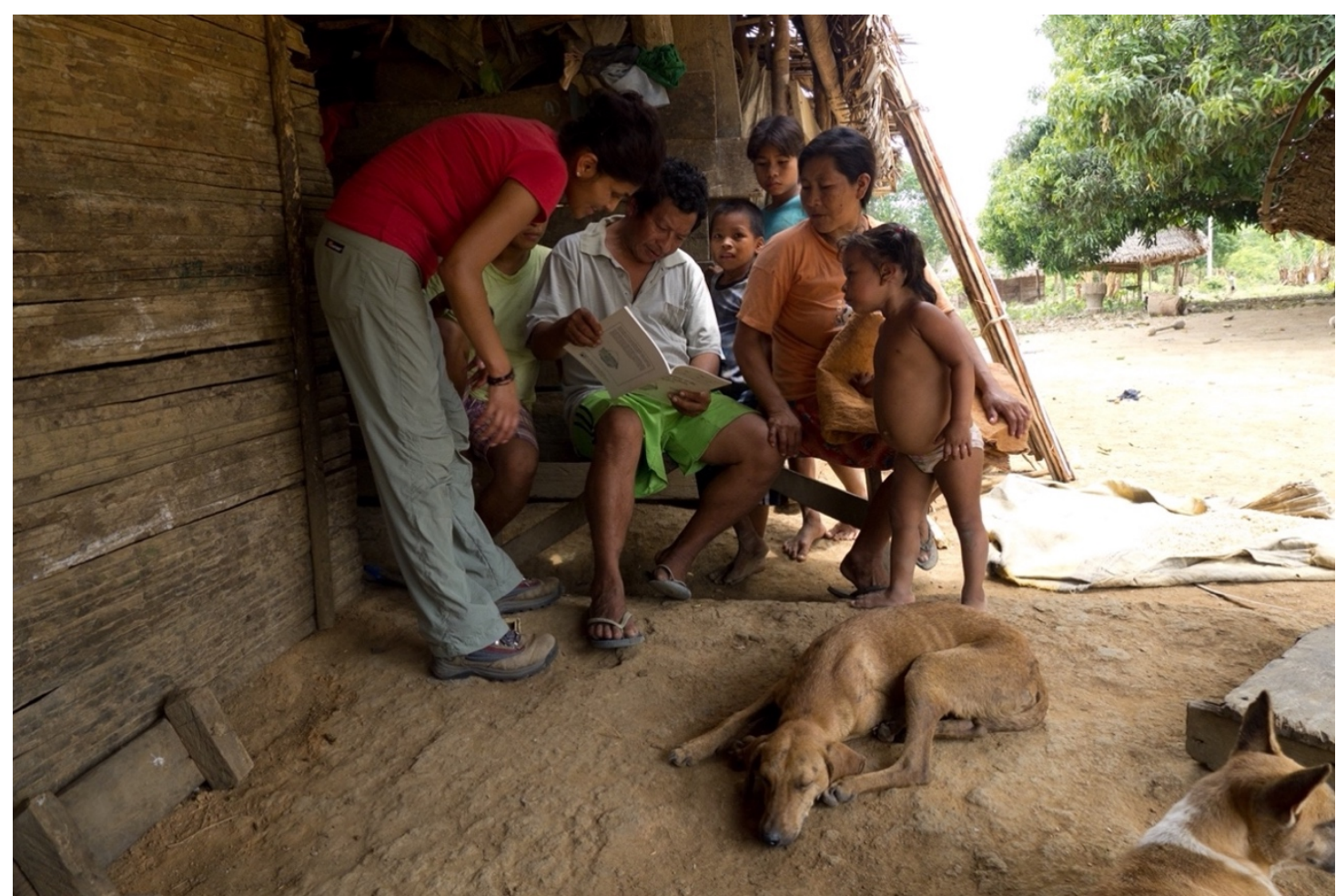

With the family of Pae Chavez, in the Chacobo indigenous community of Alto Ivon (Bolivia), reviewing the books that were returned with their traditional knowledge, 2011 (Photo RW Bussmann).

\section{What made you choose your research themes in ethnobotany}

In a specialty such as ethnobotany, the diversity of topics that can be addressed by our research is so wide that choosing is always a challenge. However, in most cases the research interests are often set by the funders, and where so much information needs to be documented, I believe that many researchers cannot afford the luxury to choose.

As in the case of many ethnobotanists, my research began as a support to ecological and taxonomic investigations (Beck et al. ,2001; Fairbain et al., 2001; Moraes \& Paniagua-Zambrana, 2006; Paniagua-Zambrana, 2005), seeking to understand the ecosystems that we studied We quickly began to direct our research towards documenting ethnobotanical knowledge in general (Bussmann et al., 2016a - d, 2017a, 2018a, 2020a,b, 2021a, b; Kupradze et al., 2015; Paniagua-Zambrana et al., 2017a) as a way to have information that communities and researchers could use as conservation tools. Some of this research was aimed at providing relevant information in relation to resource 
management and the impact of its use (Moraes \& Paniagua-Zambrana, 2007; Moraes et al.., 2008; PaniaguaZambrana \& Moraes, 2009; Preston et al. ,2003).

Much of my ethnobotanical research focused on documenting the use of palm trees (Arecaceae) mainly in Latin America (Balslev et al., 2008; Bussmann \& Paniagua-Zambrana, 2014; Macía et al., 2011, 2014; Moraes et al., 2014; Paniagua-Zambrana et al., 2007, 2015, 2017b) and based on these experiences we were also able to study palm uses in other diverse regions such as Madagascar (Bussmann et al., 2015a). I applied our research in relation to palm use to identify patterns that would allow us to understand and explain the traditional knowledge associated with them both from an ecological point of view and from a socioeconomic point of view (Bussmann \& Paniagua-Zambrana, 2013; Cámara-Leret et al., 2014; Paniagua-Zambrana et al., 2014b, c, 2015, 2016).

Like many other researchers, I focused part of my research on documenting the use of medicinal plants (Bussmann et al., 2015b, 2016a,c, 2017b; Vidaurre et al., 2006). However, many of the medicinal plants are currently sold in rural and urban markets, so I also focused my studies on documenting the knowledge related to the plants that are sold in such markets (Bussmann et al., 2016e, 2018b, c; Castañeda et al., 2021; da Costa Ferreira et al., 2021), which allowed to identify problems in the identification of commercialized plant species (Bussmann et al., 2013, 2015c). Recently, during the pandemic, we tried to provide information mainly related to the taxonomy of medicinal plants used as part of the treatments against coronavirus (COVID-19) (Maldonado et al., 2020).

The study of plants used in food has been another focus of my research, especially in the Caucasus region (Bussmann et al., 2014, 2016a, b, c, 2021b), where many of our publications were also distributed in local language.

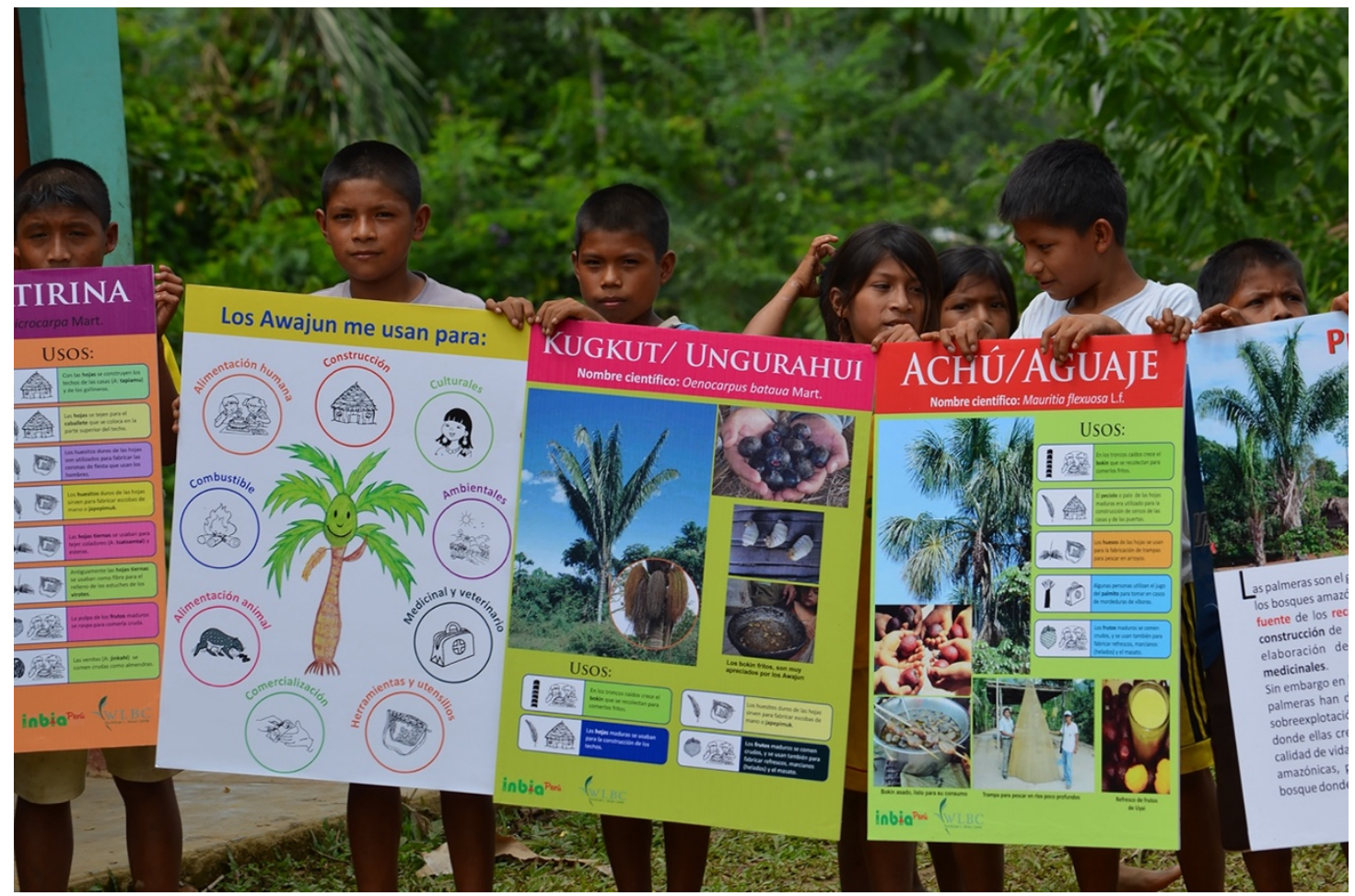

Educational material with the information documented in ethnobotanical research on the use of palm trees delivered in the schools of the Awajun community of Yamayakat in Peru, 2012 (Photo RW Bussmann).

Our investigations addressed controversial issues in our science. The loss of knowledge for example has been one of the objectives that has guided many investigations, starting with our participatory research (Bussmann et al., 2014, 2018d; Paniagua-Zambrana et al., 2018b) and we were able to address it through a different approach that allowed us to demonstrate the importance of the participation of local counterparts in our investigations and their final results (Paniagua-Zambrana et al. ,2018a, b, c). The publication of our standardized research protocols (CámaraLeret et al., 2012; Paniagua-Zambrana et al. 2010), and our experience with quantitative ethnobotany methods (Paniagua-Zambrana et al., 2018c, d; Zenderland et al., 2019) allowed us to contribute to the methodological development of ethnobotany. 
The need to have up to date information regarding the most important useful plants in different countries and regions has always been a necessity in our science. Over the last few years, we have thus started to report on important useful plants in all mountains of the world, acting as editors in. chief and volume editors for the Springer Series "Ethnobotany of Mountain Regions" (Bussmann \& Paniagua-Zambrana, 2020; Paniagua-Zambrana \& Bussmann, 2020).

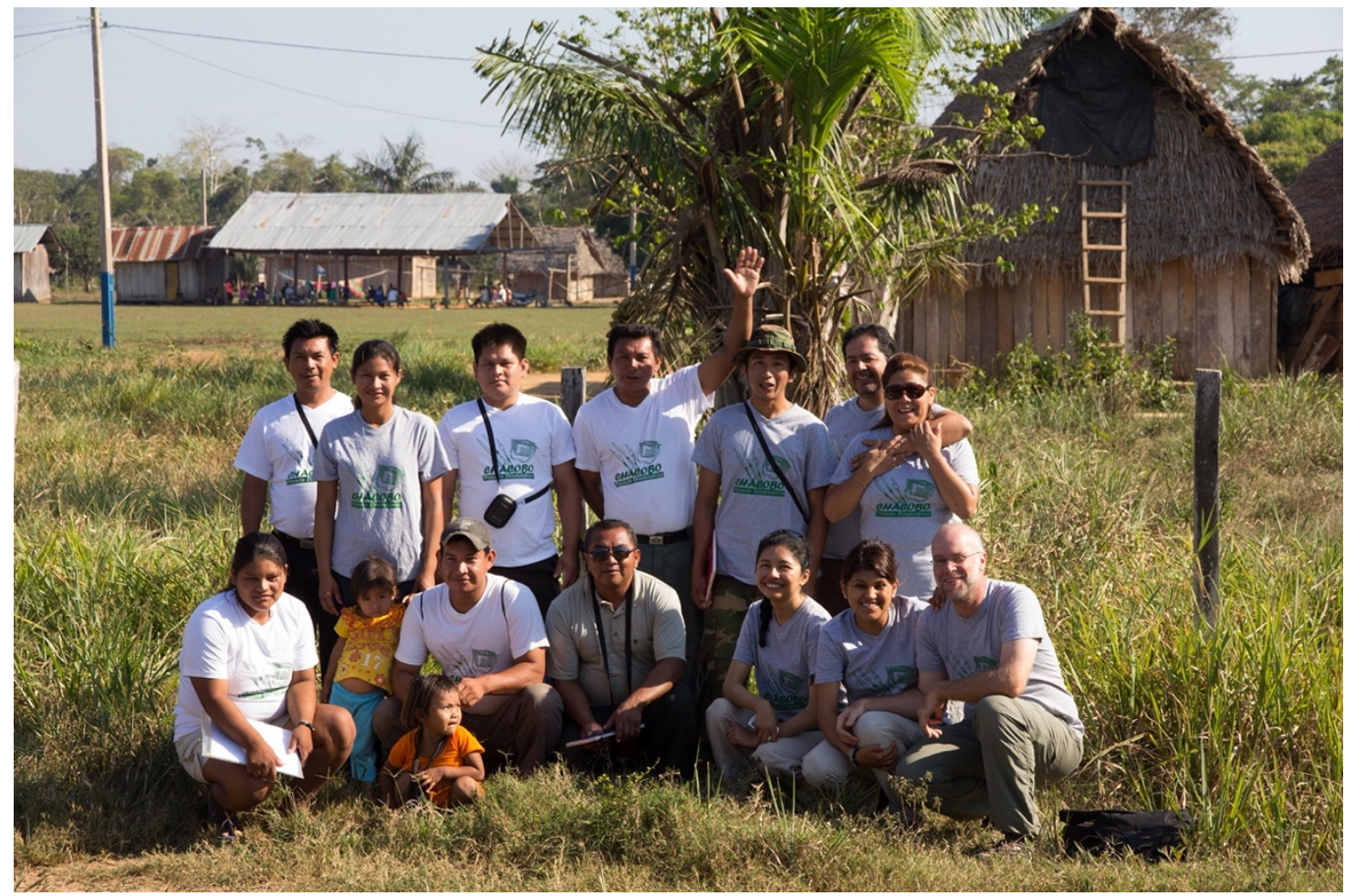

In the Chacobo community of Alto Ivon, together with the members of the Chacobo Ethnobotany Project. A participatory project that documented traditional knowledge of plant use across the indigenous group, 2013 (Photo RW Bussmann)

\section{Who was the most influential person(s) on your career? Was there ever an act of (kindness/fate) that changed your life course?}

I think that the first person who has influenced my career as an ethnobotanist has been Dr. Robin Foster, who opened the doors to the fabulous world of botany, not only in my first real field-expedition as a botanist, but by also allowing me to know other scientific institutions outside of Bolivia, and that exchange experience was very important in my training. Knowing plants from a taxonomic and ecological perspective, which has been my basic training, has been my main tool to understand the relationship that local populations establish for the use of plants, and to be able to make this information a tool in conservation.

As an ethnobotanist, without a doubt each of the people with whom we have collaborated in our research has been and are an inspiration to me. With great certainty I can say that they have not only taught me to "walk through the forest", but they have also taught me to "walk through life". The lessons that I have learned with them have allowed me to relate and learn differently both personally and professionally. The humility and detachment with which they share their knowledge, their house, their food ... their home, are lessons that I try to apply in daily life and transmit to the students with whom we work. Without their selfless collaboration, our research would not exist.

And finally, as a female scientist, without a doubt the person that I today consider my greatest inspiration, is my husband, Dr. Rainer Bussmann, with whom, for already 11 years, I have shared my life and my research as an ethnobotanist. Working together with my husband has allowed us to show that women and men can do the same job with mutual respect. And I am convinced that this can help to give value to the position of women in environments where gender differences still create conflict. 


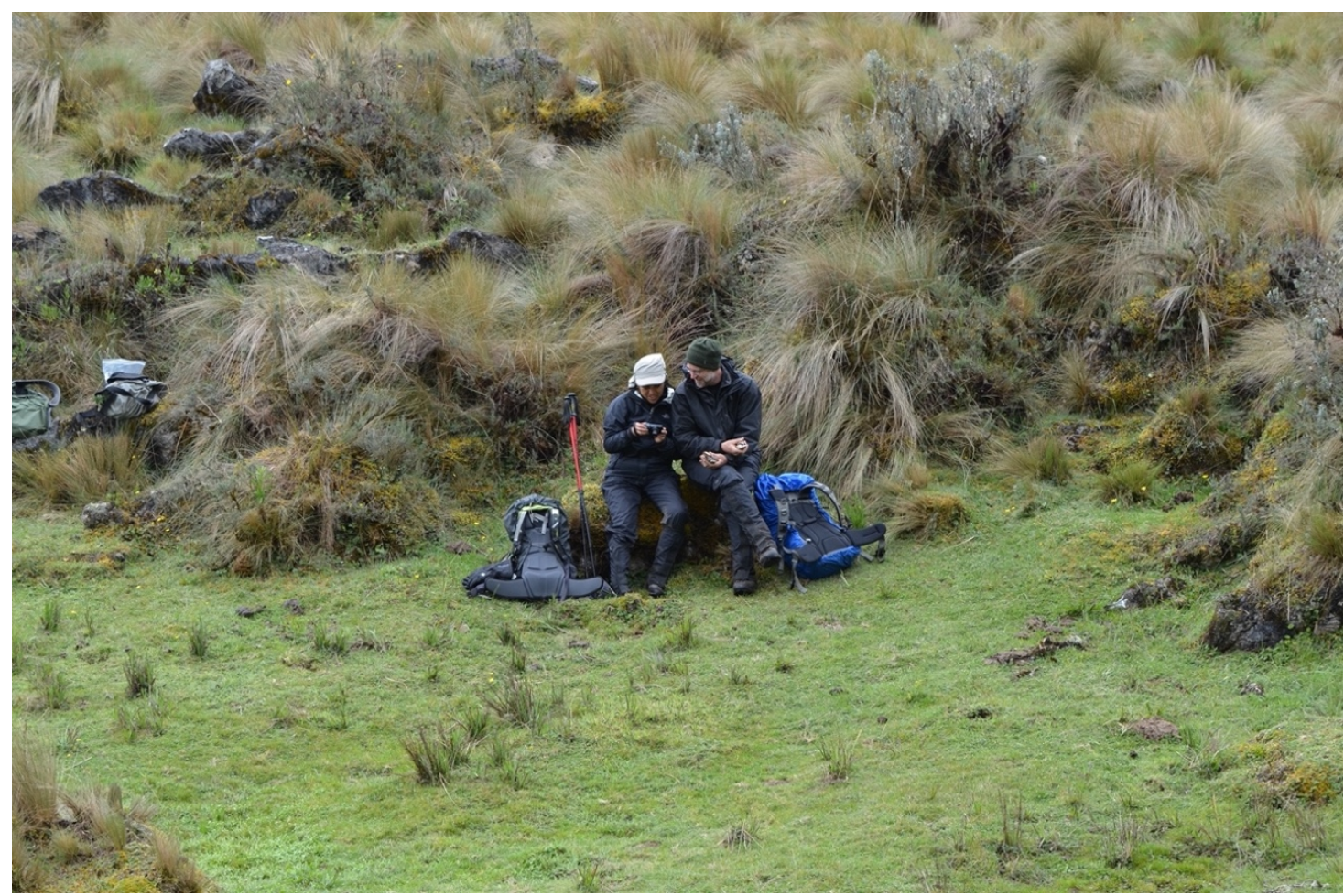

With Dr. Rainer Bussmann, my husband, in one of our botanical expeditions in the montane forests of northern Peru, 2010 (Photo Carlos Vega)

\section{What was the highlight of your career? A moment you were proud of?}

Each book delivered to a family in the communities to return their knowledge to them, with all the limitations that we still have in research in Latin America, I consider as a special achievement in my career. Seeing their surprise because we returned with "those books", reading their names as authors and finding themselves in one of the photographs that we include, are some of the reasons why we developed our research.

There have been other achievements that have also been important, such as the use of one of our publications with the Ese Eja (an indigenous group from the Peruvian-Bolivian Amazon) (Paniagua-Zambrana et al., 2012d) to declare the use of palm fibers in basket weaving as Cultural Heritage in Peru.

However, one of the achievements that has been very important came in February 2019, when I received the award for early career researchers in the world from the Organization for Women in Science for the Developing World (OWSD) and the Elsevier Foundation for my work in Ethnobotany. Only five scientists in the world receive this award each year, and I received it on behalf of Latin America and the Caribbean.

Personally, it turned out a very shocking experience for me, because from one day to the next my entire country was talking about the award, our research and ethnobotany. But the most important thing was that the award highlighted the indigenous and local communities with which we carry out our research, and their traditional knowledge as an important tool in the conservation of our cultural and natural heritage. The message was so strong that we were invited by the president of Bolivia to show him our work, many national media gave us space to talk about ethnobotany and its importance, and institutions such as the World Food Program Bolivia chose me as an image to paint a mural with my story in a school in the city of La Paz celebrating the International Day of Women. Additionally, I like to highlight an aspect that perhaps not seems important but coming from a Latin country it is very important. Receive the award highlighted the importance of family support. The family must support and believe in a scientific woman, and this often means relieving her of certain activities that our society considers exclusive responsibility of women. 


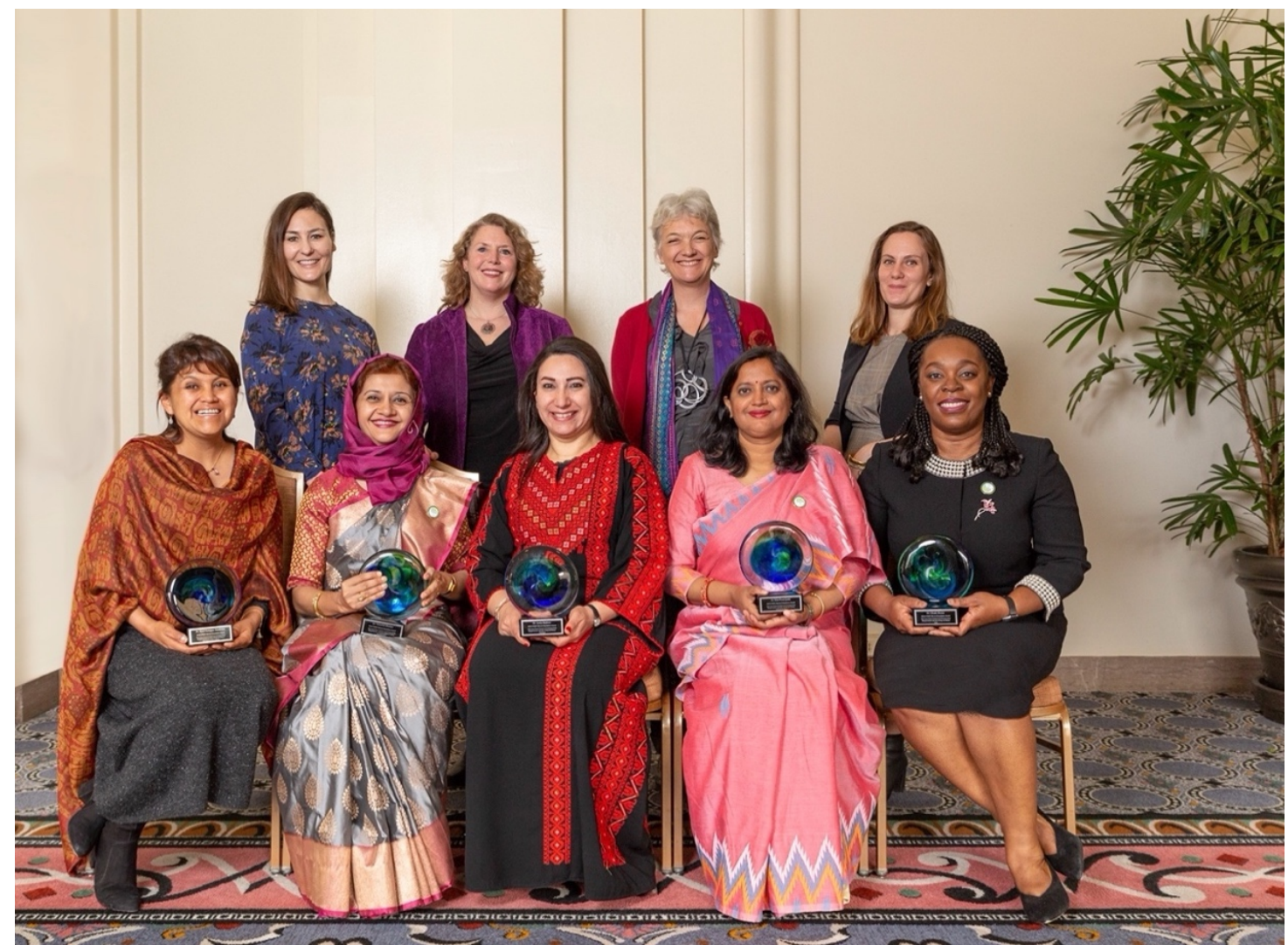

At the 2019 Organization for Women in Science for the Developing World (OWSD) and Elsevier Foundation (Washington, USA) award ceremony (Photo Alison Bert, Elservier)

- WFP Bolivia liked

WFP Bolivia @WFP_Bolivia.Mar 8

Hoy \#IWD2019 rendimos homenaje a las mujeres que nos motivan a romper barreras y soñar sin límites. Este año, reconocemos a la científica boliviana \#NarelPaniagua en un mural pintado por una artista local que permitirá a transeúntes conocer a Narel y su labor 3 \#MujeresEnCiencia

(3) Translate Tweet

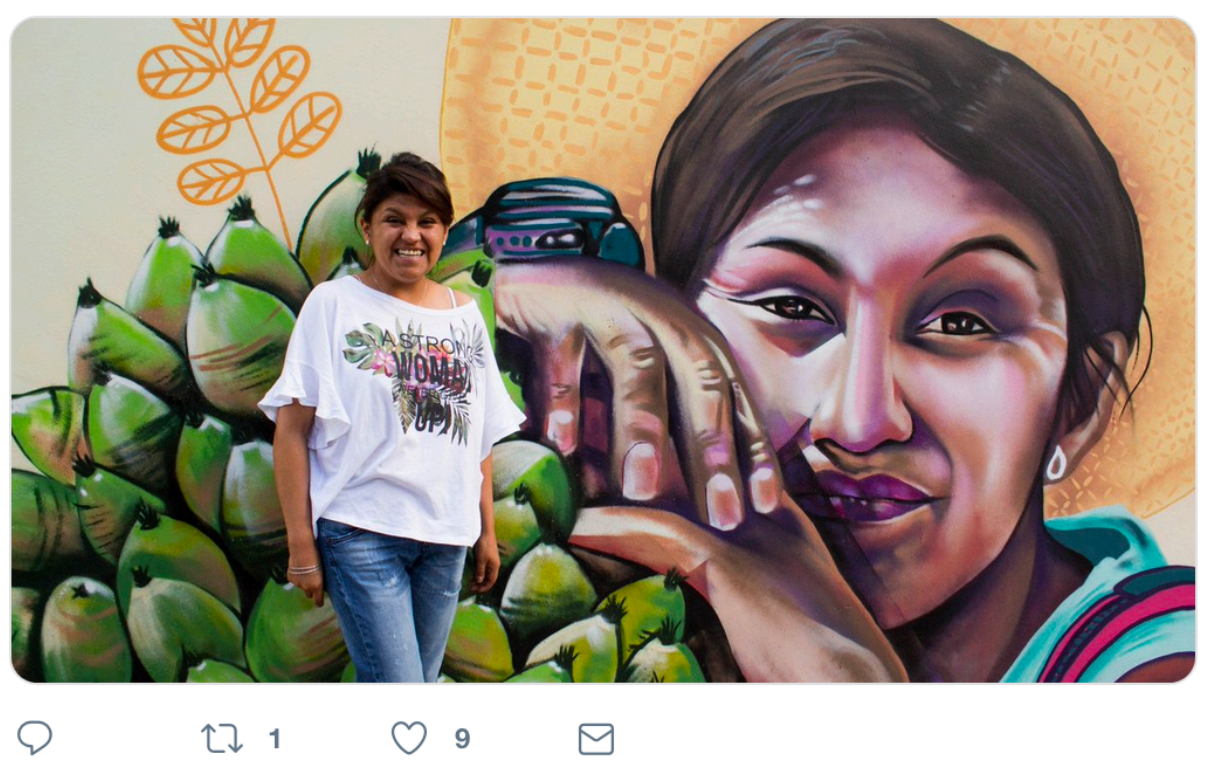

Twitter of the World Food Program Bolivia announcing the completion of the mural celebrating International Women's Day 2019 (La Paz, Bolivia). 


\section{And a lowlight? Was there ever a point you looked at yourself and thought what am I doing/how the hell did I get here?}

You can say that there were frequent low points for a long time. It is difficult to reach a community and talk to them about conserving their traditional knowledge and their resources when they themselves have many other more important needs (e.g., health, education). Thus, transmitting the importance that research had for me was frustrating. That frustration was constant for a long time, until I understood that it was me who should listen and not them. When I began to understand that we both had common interests and we needed just to identify them, when I stopped "applying protocol recipes," and recognized each community as unique, I finally began to develop research with a more participatory approach. The frustration still exists, but it is becoming easier to accept when a community gives "no" for an answer when we propose research or certain aspects of it, and to respect it.

However, although the way of conceiving ethnobotanical research is changing, certain aspects are not. Recently we were victims of a type of racism that, as a scientist, is very difficult to understand: the resistance of scientific journals to accept our collaborators in the communities as co-authors in the publications generated as products of our participatory research. Many journals that publish articles related to ethnobotany and traditional knowledge, and many reviewers and editors, are of the opinion that all authors in a publication must have some academic training. In some instances, editors and reviewers asked us if our local collaborators knew how to read and write and what their academic degree was, totally unaware that they in fact were the original owners of the knowledge that was included in these publications! As scientists we must learn to value all kinds of knowledge. Such attitudes seem predatory to me, as much or even more than the article processing charges that we as researchers are often asked to pay to publish our manuscripts, and that researchers in developing countries often cannot afford. This point of view has been highlighted in some regional publications (https://ojo-publico.com/2931/como-cientificos-debemosvalorar-todos-los-tipos-de-conocimiento) Publication practice simply must change to do what is ethically correct.

Finally, and speaking of ethics, an attitude that I see often among colleagues is the "indiscriminate" use of data obtained in the field. It is true that in many institutions our success is measured by the Impact Factor of our publications, and in the quest to have more "impact" many researchers simply "re-use" the information they have e.g. obtained for their theses, without consulting the original knowledge holders whether this use beyond the original purpose (a thesis publication) was permitted, and often not even recognizing the contribution of our indigenous and local colleagues and research participants (which are often only mentioned in the acknowledgments, if at all). Very few Journals are clear about these aspects and use them as criteria to accept or reject this type of manuscript. Others simply ignore them and contribute to the perception that ethnobotanists "steal" knowledge. We should clearly denounce this type of action, and this type of colleagues that harm the development of future research.

\section{What would be your top advice for a student of ethnobotany? (1 or 2 tips, or things you had wish you had known as a student)}

One aspect that is essential for us as ethnobotanists is remembering that we are all the same. That your position as a "researcher" does not make you better, more important, or more intelligent than the people with whom you collaborate in your research. Without THEIR PARTICIPATION and without THEIR KNOWLEDGE our research would not exist. It is important to involve local participants in all aspects of the research, from design, field work (collections and interviews) to publications, without forgetting to return the documented knowledge to its original owners. These actions empower them and generate a vision of value, which originally, they sometimes did not perceive in their traditional knowledge and in your research. Sometimes you don't realize the value of knowledge and research until you see it, and the same thing happens to them. If you go and explain your work to them, you give them the chance to participate, take their comments and suggestions and include them in the project, you make them more confident that the research we are doing can really serve them, and not just us (the researchers).

Never forget that you are working with people, and that any difference that we might have with them (perceptions, ideas, actions) must be respected. Sometimes this requires developing a lot of patience and empathy, which are very important. We must consider that we ourselves can be the main "artifact" in our research. It is important to understand that conducting ethnobotanical research is like a bread recipe, it must be adjusted according to where it is baked (due to the possible effects of altitude, pressure, toppings, etc.) and you will likely only make the best bread when you have probably burned a lot. 
And finally, the success of a good relationship is trust, and this is what we want to develop in our research. So, it is important to develop actions that lead us to it, and one of the first is NOT TO PROMISE something that you will not be able to give, just to achieve your goals.

\section{How do you see the future of ethnobotany? i.e., new trends, areas not yet researched, areas that are over researched? What do you see as the most important mission of ethnobotany for the future?}

The future of ethnobotany, in my opinion, lies in applied research. During the year of the pandemic, the importance of using medicinal plants in response to SARS-CoV-2 has resurfaced, but an aspect that has always been highlighted in ethnobotanical research has been neglected, and that is food. Local communities consider that good health is in a good diet, which involves taking care of their resources in their own ecosystems, what is known today as Food Security, these are aspects that will undoubtedly be important to know and understand better (Pieroni et al., 2020).

Knowledge is the way in which people relate to the environment and what allows us to adapt to it, which means that knowledge is not static. In this last aspect lies the importance of studying it continuously. Local communities and indigenous peoples have traditional knowledge that allows them to "relate" to nature and this knowledge goes far beyond just using it for their benefit. Understanding this relationship can allow us to understand what "quality of life" is for them. Their relationship involves many other aspects that we must consider when we speak of traditional knowledge. Understanding these and applying them as a tool to improve their quality of life is essential considering that they are an integral part of the natural and cultural ecosystems that we want to conserve.

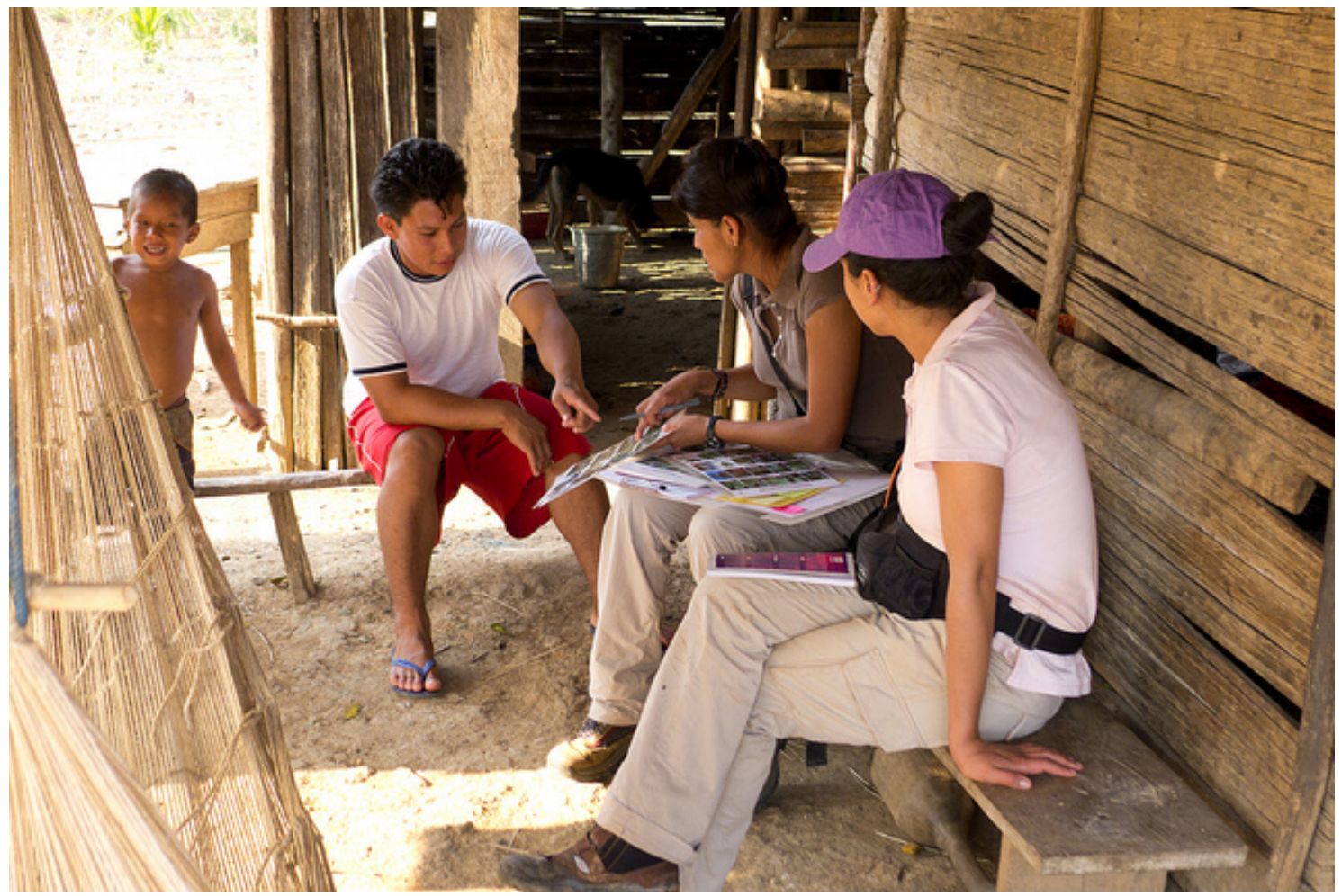

Interviewing a young Chacobo indigenous, accompanied by a student who learns to conduct interviews. Bolivia, 2010 (Photo RW Bussmann).

\section{Favorite ethnobotanical researcher?}

A colleague and friend that I admire for her dedication and commitment to ethnobotany, but also to the people she collaborates with is Dra. Ina Vandebroek at The University of the West Indies, Mona in Jamaica.

Almost at the end of the world, in Bariloche, there is Dra. Ana Ladio at INBIOMA who develops fabulous research that involves indigenous groups in her region and has trained many of the future ethnobotanists in Argentina.

And in Bolivia, my country, I deeply admire and respect Dr. Stephan Beck, founder of the Herbario Nacional de Bolivia, who is not an ethnobotanist, nor is he Bolivian (he is German) but who has a passion for botany and for 
Bolivia that is inspiring. Without all that he taught me about botany and ecology as my professor at the university and later as my colleague in our research, I could not be the ethnobotanist that I am.

\section{Have you any 'must read' ethnobotany books?}

Very difficult question to answer. I don't think I have any, because all or most of these books have been written from the perspective / experience / knowledge / needs of the author (s). So "all" provide valuable and important information and should be read with that vision. The important thing, from my point of view, are the "complementary books" to your ethnobotanical research, and a good book about the history and culture of the place where you are going to work, can be essential.

\section{What do you do in your downtime? Any secret hobbies?}

I don't think I have activities that can be identified with "my free time." All the activities I do at home or outside have some connection with ethnobotany. In some way it is always a way of learning or applying what has been learned.

Cooking is one of the passions that I share with my husband, it is a ritual that allows us to connect with the product, its origin, and through the recipe with different cultures. It's a great way to experiment, eat delicious, and practice applied ethnobotany at home.

I really like to do things with my hands. Once I used dry leaves to make postcards that I gave to my friends on special occasions, I also do origami as a way to relax and test my patience. During the year of the pandemic, I really enjoyed knitting, for now with two needles and with wool, but hopefully one day I can learn to weave baskets using natural fibers and thus I can replicate the baskets that I have been collecting in all the communities and countries that I have been able to know.

And together with my husband I learned to enjoy dogs, and now I can't imagine a life without them.

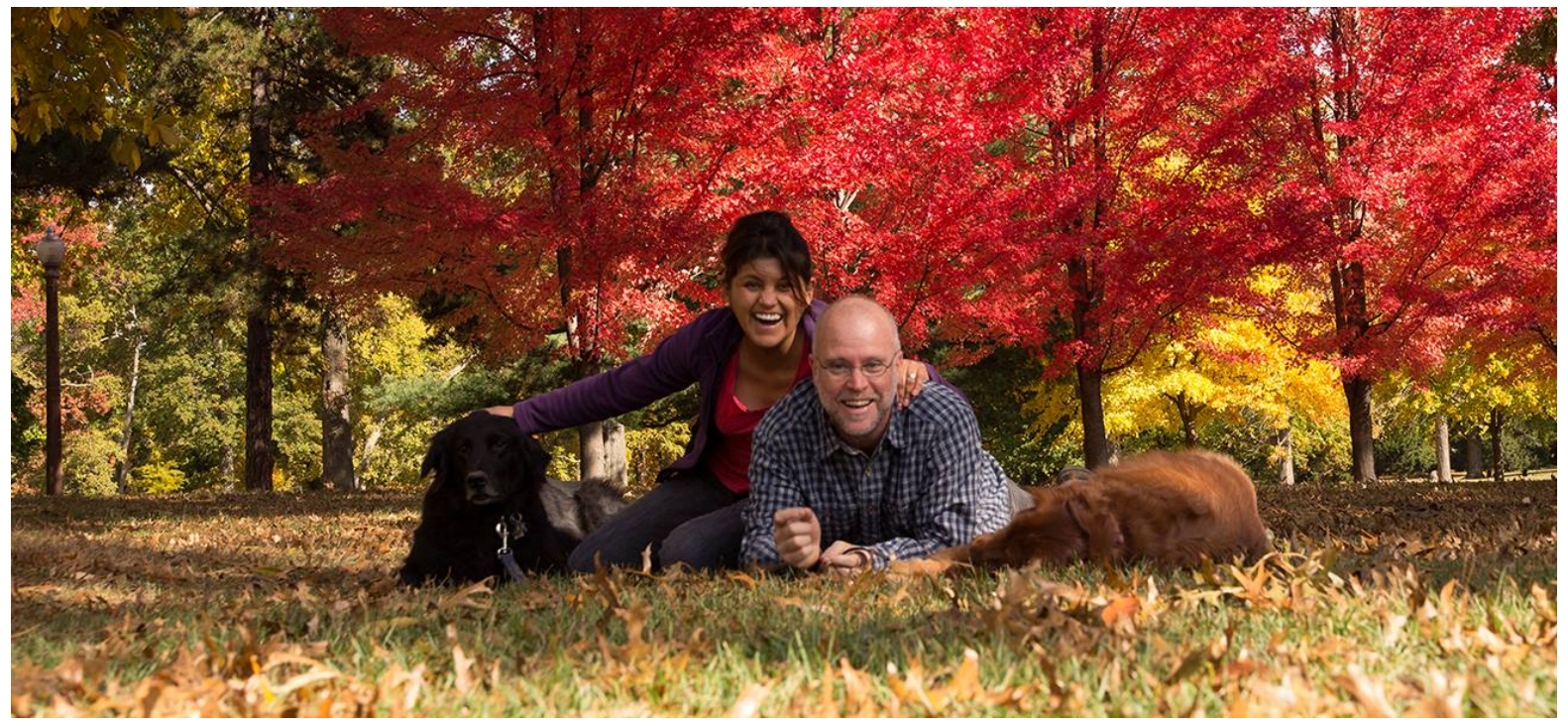

Rainer W. Bussmann and Narel Paniagua-Zambrana with Ravi and Leo, St. Louis, 2014 (Photo RW Bussmann)

\section{What is your favorite plant and why?}

My favorite plant is Attalea princeps Mart, The palm that made me have my first approach to ethnobotany and that has been identified as one of the most used in tropical forests. I was so passionate about studying it that for a long time many colleagues only identified me by its local name in Bolivia, Motacu. It is the palm tree that I chose to show with me in the mural made by the World Food Program in Bolivia. 


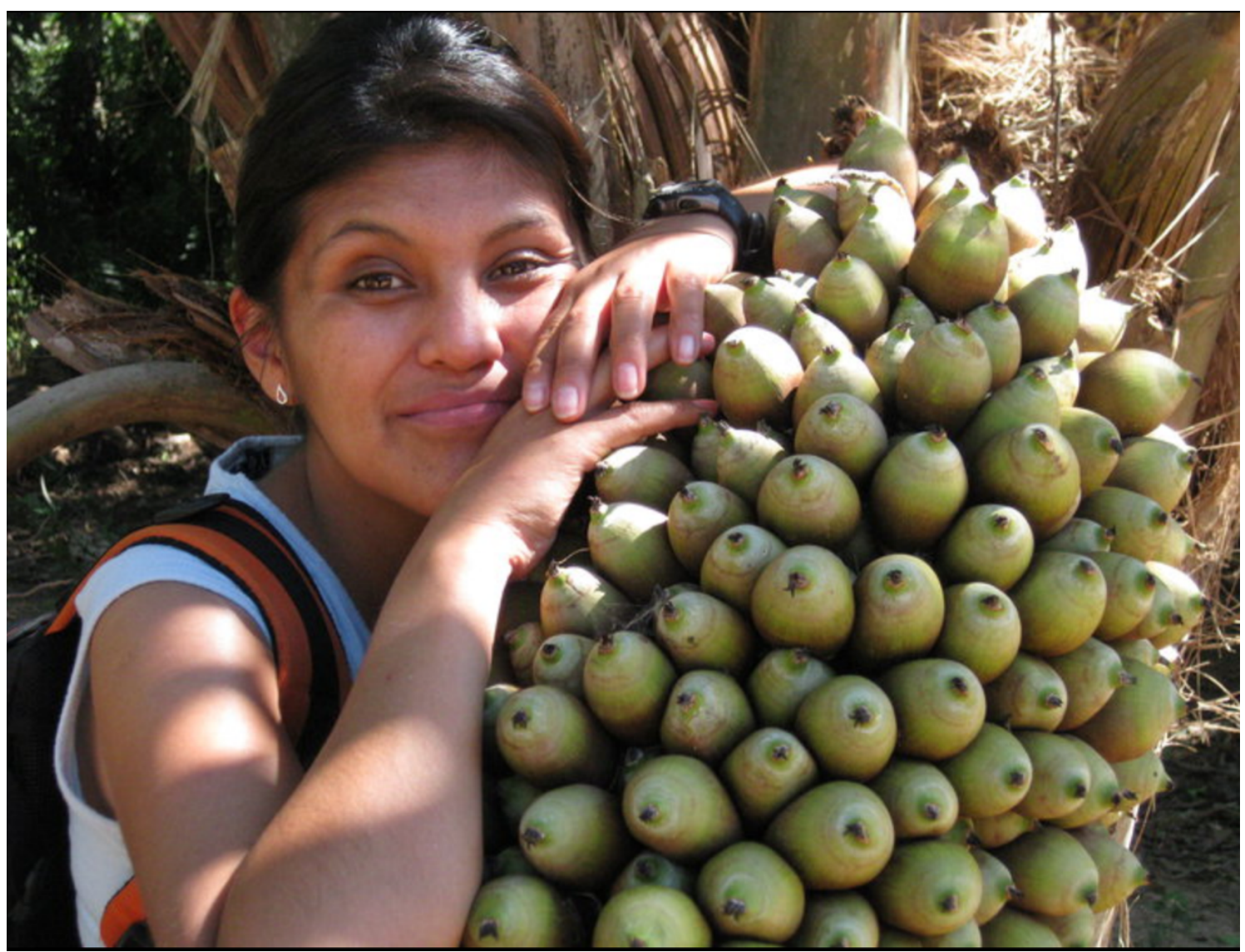

During my PhD work I "hugged" a bunch of fruits from Attalea princes Mart. in Riberalta, Bolivia, 2010. (Photo E. Blacutt).

And a second plant has been a "gift" that my husband gave me. Blakea nareliana (Melastomataceae) is a small tree, endemic to the cloud forest area in the Huallaga area in the Andes of northern Peru, collected in the place where we carried out our first field expedition together in 2010.

\section{Declarations}

List of abbreviations: N/A

Ethics approval and consent to participate: Narel Paniagua-Zambrana authorized the interview and read and approved the text.

Consent for publication: All persons shown in any image gave their permission to have the images published. All image authors agreed to have the images published.

Availability of data and materials: All the data are in manuscript and supporting documents.

Author's contributions: The author conducted the interview and wrote the article.

Funding: This study did not receive any specific grant from funding agencies in the public, commercial, or not-forprofit sectors.

Conflict of interest: The authors declares that they have no conflict of interest. 


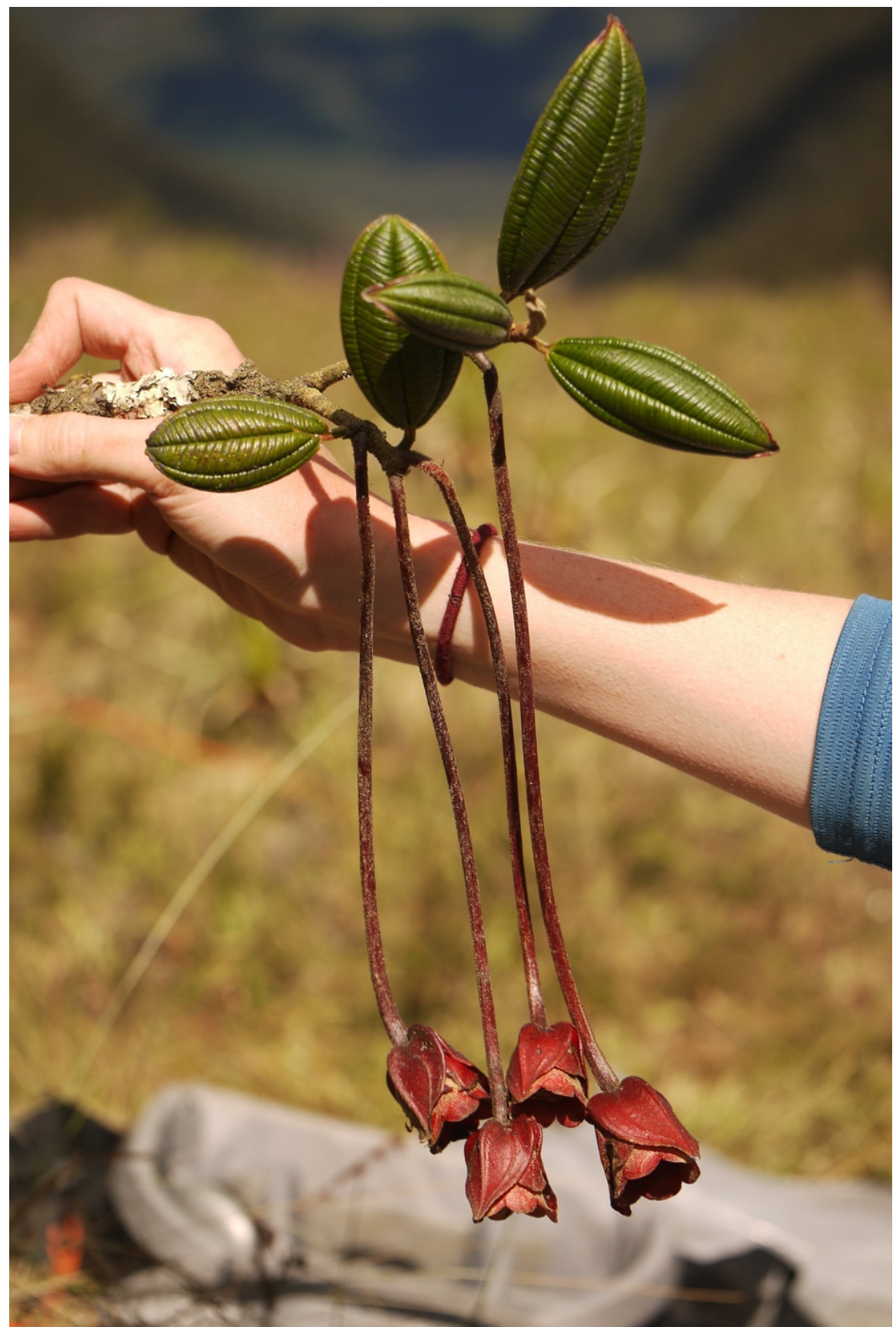

Blakea nareliana (Melastomataceae) collected in the cloud forest zone in the Huallaga River region in the Andes of northern Peru, 2010. (Photo RW Bussmann).

\section{Literature cited}

Balslev H, Grandez C, Paniagua-Zambrana NY, AL, Hansen SL. 2008. Palmas (Arecaceae) útiles en los alrededores de Iquitos, Amazonía Peruana. Revista Peruana de Biología 15 (supl. 1): 121- 132

Balslev H, Paniagua-Zambrana NY, Blacutt E, Moraes RM, Macía J, Parada, A, Inturias Y, Perez Z, Teran J, Aliaga .M, Grandez C, Serralta A. 2012. Palmeras de los Leco y sus usos. Herbario Nacional de Bolivia, Universidad Mayor de San Andrés, La Paz.

Beck S, Paniagua-Zambrana NY, Yevara M, Liberman M. 2001. La vegetación y uso de la tierra en el altiplano y los valles en el oeste del departamento de Tarija, Bolivia. In: Historia, Ambiente y Sociedad en Tarija, Bolivia. Beck, S, N. Paniagua \& D. Preston (eds.). Instituto de Ecología, UMSA/Escuela de Geografía/Universidad de Leeds. Ed. Grafica Latina. La Paz, Bolivia.

Bussmann RW, Paniagua-Zambrana NY. 2013. Facing global markets - usage changes in Western Amazonian plants: 
the example of Euterpe precatoria Mart. and E. oleracea Mart. Acta Societatis Botanicorum Poloniae 1(4): $257-261$

Bussmann RW, Paniagua-Zambrana NF, Rivas Chamorro M, Molina Moreira N, Cuadros Negri ML, Olivera J. 2013. Peril in the market - classification and dosage of species used as anti-diabetics in Lima, Peru. Journal of Ethnobiology and Ethnomedicine 9:37

Bussmann RW, Paniagua-Zambrana NY. 2014. Special Issue: Palms and Communities in the Andes and Amazon of Bolivia and Peru. Ethnobotany Research and Applications 13(1).

Bussmann RW, Paniagua-Zambrana NY, Sikharulidze S, Kikvidze Z, Kikodze D, Jinjikhadze T, Shanshiashvili T, Chelidze D, Batsatsashvili K, Bakanidze N. 2014. Wine, Beer, Snuff, Medicine, and Loss of Diversity - Ethnobotanical travels in the Georgian Caucasus. Ethnobotany Research and Applications 12: 237-312

Bussmann RW, Paniagua-Zambrana NY, Kuhlman A, Fakotoarivony F, Razanatsima A, Rasoaviety LG, Rakotoarivelo N, Razafitsalama JL, Randrianasolo A. 2015a. Palms and Palm Use in Ambalabe, a Community in Eastern Madagascar. Ethnobotany Research and Applications 14:017-026

Bussmann RW, Paniagua-Zambrana NY, Castañeda Sifuentes RY, Prado Velazco IA, Mandujano J. 2015ab Health in a Pot-The Ethnobotany of Emolientes and Emolienteros in Peru. Economic Botany 69(1): 83-88

Bussmann RW, Paniagua-Zambrana NY, Moya Huanca AL. 2015c. Dangerous Confusion "Cola de Caballo" Horsetail, in the Markets of La Paz, Bolivia. Economic Botany 69(1): 89-93

Bussmann RW, Paniagua-Zambrana NY, Sikharulidze S, Kikvidze Z, Kikodze D, Tchelidze D, Batsatsashvili K, Hart RE. 2016a. Medicinal and food plants of Svaneti and Lechkhumi, Sakartvelo (Republic of Georgia), Caucasus. Medicinal and Aromatic Plants 5:5

Bussmann RW, Paniagua-Zambrana NY, Sikharulidze S, Kikvidze Z, Kikodze D, Tchelidze D, Khutsishvili M, Batsatsashvili K, Hart RE, Pieroni A. 2016b. Your poison in my pie - the use of Potato (Solanum tuberosum L.) leaves in Sakartvelo, Georgia (Caucasus) and Gollobordo, Eastern Albania. Economic Botany 70(4): 431-437

Bussmann RW, Paniagua-Zambrana NY, Sikharulidze S, Kikvidze Z, Kikodze D, Tchelidze D, Khutsishvili M, Batsatsashvili K, Hart RE. 2016c. Medicinal and food plants of Svaneti and Lechkhumi, Sakartvelo (Republic of Georgia), Caucasus. Medicinal and Aromatic Plants 5:266, doi: 10.4172/2167-0412.1000266

Bussmann RW, Paniagua-Zambrana NY, Sikharulidze S, Kikvidze Z, Kikodze D, Tchelidze D, Khutsishvili M, Batsatsashvili K, Hart RE. 2016d. A comparative ethnobotany of Khevsureti, Samtskhe-Javakheti, Tusheti, Svaneti, and Racha-Lechkhumi, Republic of Georgia (Sakartvelo), Caucasus. Journal of Ehnobiology and Ethnomedicine 12:43. doi: 10.1186/s13002-016-0110-2

Bussmann RW, Paniagua-Zambrana NY, Moya Huanca, LA, Hart RE. 2016e. Changing markets - medicinal plants in the markets of La Paz and El Alto, Bolivia. Journal of Ethnopharmacology 193: 76-95. doi: 10.1016/j.jep.2016.07.074

Bussmann RW, Paniagua-Zambrana NY, Sikharulidze S, Kikvidze Z, Kikodze D, Tchelidze D, Batsatsashvili K, Hart RE. 2017a. Ethnobotany of Samtskhe-Javakheti, Sakartvelo (Republic of Georgia), Caucasus. Indian Journal of Traditional Knowledge 16(1): 7-24

Bussmann RW, Paniagua-Zambrana NY, Sikharulidze S, Kikvidze Z, Kikodze D, Tchelidze D, Batsatsashvili K, Hart RE. 2017b. Plants in the spa - the medicinal plant market of Borjomi, Sakartvelo (Republic of Georgia), Caucasus. Indian Journal of Traditional Knowledge 16(1): 25-34

Bussmann RW, Paniagua-Zambrana NY, Sikharulidze S, Kikvidze Z, Kikodze D, Tchelidze D, Batsatsashvili K, Hart RE. 2018a. Unequal brothers - Plant and fungal use in Guria and Racha, Sakartvelo (Republic of Georgia), Caucasus. Indian Journal of Traditional Knowledge 17(1): 7-33

Bussmann RW, Paniagua-Zambrana NY, Romero C, Hart RE. 2018b. Astonishing diversity - the medicinal plant markets of Bogotá, Colombia. Journal of Ethnobiology and Ethnomedicine 14(1):43. doi: 10.1186/s13002-018-02418

Bussmann RW, Paniagua-Zambrana NY, Romero C, Hart RE. 2018c. No consensus in "traditional" medicine Medicinal plants and their uses in the markets of Bogotá (Colombia), La Paz/El Alto (Bolivia) and Trujillo/Chiclayo (Perú). Indian Journal of Traditional Knowledge 17(3): 494-498 
Bussmann RW, Paniagua-Zambrana NY, Wood N, Ole Njapit S, Ole Njapit JN, Ene Osoi GE, Kasoe SP. $2018 \mathrm{~d}$. Knowledge loss and change between 2002 and 2017 - a revisit of plant-use of the Maasai of Sekenani Valley, Maasai Mara, Kenya. Economic Botany 72(2): 207-216

Bussmann RW, Paniagua-Zambrana NY (Eds.) 2020. Ethnobotany of Mountain Regions. Springer International Publishing: Cham.

Bussmann, R.W, Paniagua-Zambrana NY, Sikharulidze S, Kikvidze Z, Kikodze, D, Tchelidze, D, Khutsishvili M, Batsatsashvili K, Hart, R.E. 2020a. An ethbobotany iof Kahkheti and Kvemo Kartli, Sakartvelo (Republic of Georgia), Caucasus. Ethnobotany Research and Applications 19(35) doi: 10.32859/era.19.47.1-28

Bussmann RW, Paniagua-Zambrana NY, Sikharulidze S, Kikvidze Z, Darchidze M, Manvelidze Z, Ekhvaia J, Kikodze D, Tchelidze D, Khutsishvili M, Batsatsashvili K, Hart RE. 2020b. Form the sea to the mountains - plant use in Ajara, Samegrelo and Kvemo Svaneti, Sakartvelo (Republic of Georgia), Caucasus. Ethnobotany Research and Applications 20(9) doi: 10.32859/era.20.9.1-34

Bussmann RW, Paniagua-Zambrana NY, Sikharulidze S, Kikvidze Z, Kikodze D, Tchelidze D, Batsatsashvili K, Hart RE. 2021a. An ethnobotany of Upper Imereti, Ukana Pshavi, Meshketi and Pankisi gorge, Sakartvelo (Republic of Georgia), Caucasus. Bocconea 29:33-54. doi: 10.732010.7320/Bocc29.001

Bussmann RW, Paniagua-Zambrana NY, Ur Rahman I, Kikvidze Z, Sikharulidze S, Kikodze D, Tchelidze D, Khutsishvili M, Batsatsashvili K 2021b. Unity in diversity - Food plants and fungi of Sakartvelo (Republic of Georgia), Caucasus. Journal of Ethnobiology and Ethnomedicine 17(72). doi: 10.1186/s13002-021-00490-9

Cámara-Leret R, Paniagua-Zambrana NY, Macía MJ. 2012. Standard Protocol for Gathering Palm Ethnobotanical Data and Socioeconomic Variables across the Tropics. pp. 41-72. In: Ponman B, Bussmann RW (eds.): Medicinal plants and the legacy of Richard E. Schultes. Proceedings of the Botany 2011 Richard E Schultes Symposium. Graficart, Trujillo.

Cámara-Leret, R, Paniagua-Zambrana NY, Balslev H, Barfod A, Copete JC, Macía MJ. 2014. Ecological community traits and traditional knowledge shape palm ecosystem services in northwestern South America. Forest Ecology and Management 334:28-42

Castañeda R, Gutiérrez H, Aponte H, Ocampo IZ, Bussmann RW, Paniagua-Zambrana NY. 2021. The trade of wild medicinal plants in the andean district of Lircay, Huancavelica, Perú: A comparative study. Ethnobotany Research and Applications 21(22):1-32. doi: 10.32859/era.21.22.1-32

da Costa Ferreira E, Paiva de Lucena RF, Bussmann RW, Paniagua-Zambrana NY, Dias da Cruz D. 2021. Temporal assessment of the medicinal plant trade in public markets of the state of Paraíba, northeastern Brazil. Journal of Ethnobiology and Ethnomedicine 17:70. doi: 10.1186/s13002-021-00496-3

Fairbarin J, Preston D, Paniagua-Zambrana NY, Mass G, Yevara M, Beck S. 2001. Pastoreo y cambios ambientales en el altiplano de Tarija, Bolivia. En Historia, Ambiente y Sociedad en Tarija, Bolivia. Beck, S, N. Paniagua \& D. Preston (eds.). Instituto de Ecología, UMSA/Escuela de Geografía/Universidad de Leeds. Ed. Grafica Latina. La Paz, Bolivia.

Kupradze I, Jorjadze A, Arabidze A, Beltadze T, Batsatsashvlli K, Paniagua-Zambrana NY, Bussmann RW. 2015. Ethnobiological study of Svaneti Fungi and Lichens: History of research, diversity, local names and traditional use. American Journal of Environmental Protection 4(3-1): 101-110

Macía MJ, Armesilla J, Cámara-Leret R, Paniagua-Zambrana NY, Villalba S, Balslev H, Manuel Pardo-de-Santayana M. 2011. Palm Uses in North-western South America: a Quantitative Review. The Botanical Review 77(4): $462-570$

Macía MJ, Cámara Leret R, Paniagua-Zambrana NY. 2014. Uso de palmas por poblaciones locales. pp.57-86. In: Balslev H, Macía MJ, Navarrete H (Eds.). Cosecha de palmas en el noroeste de Sudamérica - las bases científicas para su manejo y conservación. Pontificia Universidad Católica de Ecuador, Quito.

Maldonado C, Paniagua-Zambrana NY, Bussmann RW, Zenteno-Ruiz FS, Fuentes AF. 2020. La importancia de las plantas medicinales, su taxonomía y la búsqueda de la cura a la enfermedad que causa el coronavirus (COVID-19). The importance of medicinal plants, their taxonomy and the search for a cure for the disease caused by the coronavirus (COVID-19). Ecología en Bolivia 55(1):1-5

Moraes M, Paniagua-Zambrana NY. 2006. Biología y Ecología de la Jatata (Geonoma deversa). In: Ergueta et al. (Eds.) Jatata: Un recurso valioso para los habitantes del bosque tropical. Proyecto TROPICOS. pp. 57-72 
Moraes-R M, Paniagua-Zambrana NY. 2007. Tasas productivas del motacú (Attalea phalerata, Arecaceae) hacia el manejo sostenible en Bolivia. Pp. 1637. In: Feyen J, Aguirre LF, Moraes-R M (eds.) Memorias Congreso Internacional sobre Desarrollo, Medio Ambiente y Recursos Naturales: Sostenibilidad a Múltiples Niveles y Escalas, 11-13 julio 2007, Universidad Mayor de San Simón, Cochabamba.

Moraes-R M, Paniagua-Zambrana NY Velarde-V MJ. 2008. Bases biológicas para el manejo forestal de productos derivados de palmeras de Bolivia. Pp. 172-175. In: Memorias IV Reunión Nacional sobre Investigación Forestal, PROMAB, Cobija.

Moraes, M, Paniagua-Zambrana NY, Cámara Leret R, Balslev B, Macía MJ. 2014. Palmas útiles de Bolivia, Colombia, Ecuador y Perú. pp. 87-102. In: H. Balslev, M.J. Macía y H. Navarrete, editores. Cosecha de palmas en el noroeste de Sudamérica - las bases científicas para su manejo y conservación. Pontificia Universidad Católica de Ecuador, Quito.

Paniagua-Zambrana NY, Comunidad San José de Uchupiamonas. 2001. Guía Ilustrada de plantas leñosas útiles de la comunidad San José de Uchupiamonas (Provincia Abel Iturralde, Departamento de La Paz, Bolivia). Liga de Defensa del Medio Ambiente, Herbario Nacional de Bolivi., La Paz, Bolivia.

Paniagua-Zambrana NY. 2005. Riqueza y uso de las palmas en la región del Madidi, noroeste del departamento de La Paz (Bolivia). Ecología en Bolivia 40(3): 265-280

Paniagua-Zambrana NY, Byg A, Svenning JC, Moraes, M, Grandez C, Balslev H. 2007. Diversity of palm uses in the western Amazon. Biodiversity and Conservation (16): 2771-2787

Paniagua-Zambrana NY, Moraes M. 2009. Hacia el manejo del motacú (Attalea phalerata, Arecaceae) bajo diferente tipo de cosecha (Riberalta, depto. Beni, NE Bolivia): Estructura y densidad poblacional. Revista GAB 4:17-23

Paniagua-Zambrana NY, Macía MJ, Cámara-Leret R. 2010. Protocolo para la toma de datos etnobotánicos de palmeras y variables socioeconómicas en comunidades rurales. Ecología en Bolivia 45(3): 44-68

Paniagua-Zambrana NY, Bussmann RW, Blacutt E, Macia MJ. (eds.). 2011. Los Chácobo y las Palmeras. Herbario Nacional de Bolivia, William L. Brown Center, Missouri Botanical Garden. Graficart, Trujillo, Perú.

Paniagua-Zambrana NY, Bussmann RW, Vega C, Téllez C, Macía MJ. 2012a. Nuestro conocimiento y uso de las palmeras - una herencia para nuestros hijos. Comunidades Llaquash, San Martín, Perú. Herbario Nacional de Bolivia, William L. Brown Center, Missouri Botanical Garden. Graficart, Trujillo, Perú.

Paniagua-Zambrana NY, Bussmann RW, Macía MJ. 2012b. El bosque SI tiene valor - el uso de palmeras en las comunidades campesinas e indígenas de la región de Inambari, Madre de Dios, Perú. Graficart, Trujillo, Perú.

Paniagua-Zambrana NY, Bussmann RW, Vega C, Téllez C, Macía M.J. 2012c. Kampanak se usa para el techo pero ya no hay - Uso y conservación de palmeras entre los Awajún, Amazonas, Perú. Herbario Nacional de Bolivia, William L. Brown Center, Missouri Botanical Garden. Graficart, Trujillo, Perú.

Paniagua-Zambrana NY, Bussmann RW, Macía MJ. 2012d. El conocimiento de nuestros ancestros - los Ese Eja y su uso de palmeras, Madre de Dios, Perú. Herbario Nacional de Bolivia, William L. Brown Center, Missouri Botanical Garden. Graficart, Trujillo, Perú.

Paniagua-Zambrana NY, Bussmann RW, Blacutt, E, Macía MJ. 2012e. Conservando nuestros bosques: conocimiento y uso de las palmeras en las comunidades campesinas del norte de Bolivia. Herbario Nacional de Bolivia, William L. Brown Center, Missouri Botanical Garden. Plural Editores, La Paz, Bolivia.

Paniagua-Zambrana NY, Bussmann RW, Vega C, Tellez C. 2014a. Los Chacobo y su historia en el siglo XX. Herbario Nacional de Bolivia, William L. Brown Center, Missouri Botanical Garden. Graficart, Trujillo, Perú.

Paniagua-Zambrana NY, Cámara Leret R, Bussmann RW, Macía MJ. 2014b. The influence of socioeconomic factors on traditional knowledge: a cross scale comparison of palm use in northwestern South America. Ecology and Society 19(4): 9

Paniagua Zambrana, N, Cámara-Leret R, Macía, MJ. 2014c. Patrones de uso de las palmeras en bosques amazónicos y montanos de Bolivia: Género, edad, grado de educación e idioma. pp. 127-132. In: M. Moraes, editores. Palmeras útiles de Bolivia - Las especies mayormente aprovechadas para diferentes fines y aplicaciones. Herbario Nacional de Bolivia, Universidad Mayor de San Andrés, Plural editores, La Paz. 
Paniagua-Zambrana NY, Cámara-Leret R, Macía MJ. 2015 Patters of Medicinal use of palms across Northwestern South America. 2015. The Botanical Review 81(4): 317-415

Paniagua-Zambrana NY, Cámara-Leret R, Macía MJ. 2016. Understanding transmission of traditional knowledge across north-western South America: a cross-cultural study in palms (Arecaceae). Botanical Journal of Linnean Society 182(2):480-504. doi: 10.1111/boj.12418

Paniagua-Zambrana NY, Bussmann RW, Hart RE, Moya Huanca AL, Ortiz Soria G, Ortiz Vaca M, Ortiz Álvarez D, Soria Morán J, Soria Morán M, Chávez S, Chávez Moreno B, Chávez Moreno G, Roca O, Siripi E. 2017a. Traditional knowledge hiding in plain sight - twenty-first century ethnobotany of the Chacobo in Beni, Bolivia. Journal of Ethnobiology and Ethnomedicine 13(1). doi: 10.1186/s13002-017-0179-2

Paniagua-Zambrana NY, Bussmann RW, Macía MJ. 2017b. The socioeconomic context of the use of Euterpe precatoria Mart. and E. oleracea Mart. in Bolivia and Peru. Journal of Ethnobiology and Ethnomedicine 13(32). doi: :10.1186/s13002-017-0160-0

Paniagua-Zambrana NY, Bussmann RW (Eds.) 2017c. La etnobotánica de los Chácobo en el siglo XXI. Herbario Nacional de Bolivia, Instituto de Ecología, Universidad Mayor de San Andrés, William L. Brown Center. Missouri Botanical Garden. Graficart, Trujillo, Perú. (ISBN 978-0-9060231-5-3)

Paniagua-Zambrana NY, Bussmann RW. 2018. La Etnobotánica de los Chácobo en el Siglo XXI. Ethnobotany Research and Applications 16(2): 1-149. doi: 10.32859/era.16.2.001-149

Paniagua-Zambrana NY, Bussmann RW, Hart RE, Moya Huanca AL, Ortiz Soria G, Ortiz Vaca M, Ortiz Álvarez D, Soria Morán J, Soria Morán M, Chávez S, Chávez Moreno B, Chávez Moreno G, Roca O, Siripi E. 2018a. Who should conduct ethnobotanical studies? Effects of different interviewers in the case of the Chacobo Ethnobotany project, Beni, Bolivia. Journal of Ethnobiology and Ethnomedicine, 14(1):9. doi: 10.1186/s13002-018-0210-2

Paniagua-Zambrana NY, Bussmann RW, Hart RE, Moya Huanca AL, Ortiz Soria G, Ortiz Vaca M, Ortiz Álvarez D, Soria Morán J, Soria Morán M, Chávez S, Chávez Moreno B, Chávez Moreno G, Roca O, Siripi E. 2018b. Research methods leading to a perception of knowledge loss - one century of plant use documentation among the Chacobo in Bolivia. Economic Botany 72(1):81-93

Paniagua-Zambrana NY, Bussmann RW, Hart RE, Moya Huanca AL, Ortiz Soria G, Ortiz Vaca M, Ortiz Álvarez D, Soria Morán J, Soria Morán M, Chávez S, Chávez Moreno B, Chávez Moreno G, Roca O, Siripi E. 2018c. Research methods leading to a perception of knowledge loss - one century of plant use documentation among the Chacobo in Bolivia. Economic Botany 72(1):81-93

Paniagua-Zambrana NY, Bussmann RW, Hart RE, Moya Huanca LA, Ortiz Soria G, Ortiz Vaca M, Ortiz Álvarez D, Soria Morán J, Soria Morán M, Chávez S, Chávez Moreno B, Chávez Moreno G, Roca O, Siripi E. 2018d. To list or not to list? The value and detriment of freelisting in ethnobotanical studies. Nature Plants 4(4):201-204

Paniagua-Zambrana NY, Bussmann RW., Tellez, C, Vega, C. 2018e. La Etnobotánica de los Chácobo en el Siglo XX. Ethnobotany Research and Applications 16(1):1-393. doi: 10.32859/era.16.1.001-393

Paniagua-Zambrana NY, Bussmann RW. (Eds.) 2020. Ethnobotany of the Andes. Springer International Publishing, Cham. pp. XXXI + 1955, ISBN: 978-3-030-28932-4

Paniagua-Zambrana NY, Bussmann RW. 2021. Casos se implementación del Protocolo de_Nagoya en Bolivia: la ethnobotánica de los Chácobo Pacahuara en el siglo XXI. pp57-91. In: Delgado Burgoa, JMF, Silvestre Rojas, CA (eds). Alcances teórico metodológicos y experiencias de diálogo intercientífico en países Andino Amazónicos. Ministerio de Educación, Viceministerio de Ciencia y Tecnología, Dirección General de Ciencia y Tecnología. La Paz, Bolivia.

Pieroni A, Vandebroek I, Prakofjewa J, Bussmann RW, Paniagua-Zambrana NY, Maroyi A, Torri L, Zocchi DM, Dam ATK, Khan SM, Ahmad H, Yeşil Y, Huish R, Pardo-de-Santayana M, Mocan A, Hu X, Boscolo O, Sõukand R. 2020. Taming the pandemic? The importance of homemade plant-based foods and beverages as community responses to COVID-19. Journal of Ethnobiology and Ethnomedicine 16:75. doi: 10.1186/s13002-020-00426-9

Preston D, Fairbain J, Paniagua-Zambrana NY, Mass G. 2003. Grazing and environmental change on the Tarija Altiplano, Bolivia. Mountain Research and development 23(2): 141-148 
Vega C, Tellez C, Bussmann RW, Paniagua-Zambrana NY. 2014. Yo conozco y cuido las palmeras. William L. Brown Center, Missouri Botanical Garden. Graficart, Trujillo, Perú.

Vidaurre PJ, Paniagua-Zambrana NY, Moraes-R M. 2006. Etnobotánica en los Andes de Bolivia. pp. 224-238. In: Botánica Económica de los Andes Centrales. Moraes et al. (Eds.). Universidad Mayor de San Andrés. La Paz, Bolivia.

Zenderland J, Hart R, Bussmann RW, Paniagua-Zambrana NY, Sikharulidze S, Kikvidze Z, Kikodze, D, Tchelidze, D, Khutsishvili M, Batsatsashvili K 2019. The Use of "Use Value": Quantifying Importance in Ethnobotany. Economic Botany 73:293-303

\section{Bibliography Dr. Narel Y. Paniagua Zambrana}

\section{ARTICLES IN PEER REVIEWED JOURNALS}

Albuquerque UP, Ferreira Júnior WS, Vandebroek I, Bussmann RW, Paniagua-Zambrana NY, Ladio AH, Voeks R, Melo F, Jacob MCM, Gonçalves-Souza T, Lopez AV, Soldati GT- 2021. A Reply to Pierotti's (2018) Review of "Evolutionary Ethnobiology": Decolonizing Latin American Science. Ethnobiology Letters 12(1):79-81. doi: 10.14237/ebl.12.1.2021.1763

Araujo-M A, Bascopé F, Cardona-Peña V, De la Quintana D, Fuentes A, Jørgensen PM, Maldonado C, Miranda T, Paniagua-Zambrana NY, Seidel R. 2005. Composición florística y estructura del bosque amazónico preandino en el sector del Arroyo Negro, Parque Nacional Madidi, Bolivia. Ecología en Bolivia 40(3): 281-303.

Araujo-M A, Cardona-P V, De la Quintana D, Fuentes A, Jørgensen PM, Maldonado C, Miranda T, PaniaguaZambrana NY, Seidel R. 2005. Estructura y diversidad de leñosas del bosque amazónico preandino en el sector del Río Quendeque, Parque Nacional Madidi, Bolivia. Ecología en Bolivia 40(3): 304-324.

Araujo-M A, Jørgensen PM, Maldonado C, Paniagua-Zambrana NY. 2005. Composición florística y estructura del bosque de ceja de monte en yungas en el sector de tambo Quemado, Pelechuco. Ecología en Bolivia 40(3): 325-338.

Araujo-Murakami A, Paniagua-Zambrana NY, Cayola L, Jørgensen PM, Valdes O, Macuapa E, CalzadillaTomianovich M. 2009. Diversidad y estructura florística del bosque de llanura y palmar de pantano amazónico preandino en el sector de Chalalan, Parque Nacional Madidi, Bolivia. Kempffiana 5(1):3-27.

Balslev H, Grandez C, Paniagua-Zambrana NY, AL, Hansen SL. 2008. Palmas (Arecaceae) útiles en los alrededores de Iquitos, Amazonía Peruana. Revista Peruana de Biología 15 (supl. 1): 121- 132.

Balslev H, Pérez Durán Z, Pedersen D, Eiserhardt WL, Sanjinés Asturizaga A, Paniagua-Zambrana NY. 2012. Subandean and adjacent lowland palm communities in Bolivia. Ecología en Bolivia 47(1): 7-36

Bussmann RW, Applequist W, Paniagua-Zambrana NY. 2014. Traditional Medicine in a Global Environment. Evidence-based Complementary and Alternative Medicine 2014: 1-6.

Bussmann RW, Paniagua Zambran, NY, Vega C. 2013. Axinaea carolinae-tellezii (Melastomataceae) - another new species form Northern Peru. Arnaldoa 20 (1): 19-24.

Bussmann RW, Paniagua-Zambrana NF, Rivas Chamorro M, Molina Moreira N, Cuadros Negri ML, Olivera J. 2013. Peril in the market - classification and dosage of species used as anti-diabetics in Lima, Peru. Journal of Ethnobiology and Ethnomedicine 9:37.

Bussmann RW, Paniagua-Zambrana NY, Castañeda Sifuentes RY, Prado Velazco IA, Mandujano J. 2015. Health in a Pot-The Ethnobotany of Emolientes and Emolienteros in Peru. Economic Botany 69(1): 83-88.

Bussmann RW, Paniagua-Zambrana NY, Kuhlman A, Fakotoarivony F, Razanatsima A, Rasoaviety LG, Rakotoarivelo N, Razafitsalama JL, Randrianasolo A. 2015. Palms and Palm Use in Ambalabe, a Community in Eastern Madagascar. Ethnobotany Research and Applications 14:017-026.

Bussmann RW, Paniagua-Zambrana NY, Moya Huanca AL. 2015. Dangerous Confusion "Cola de Caballo" Horsetail, in the Markets of La Paz, Bolivia. Economic Botany 69(1): 89-93.

Bussmann RW, Paniagua-Zambrana NY, Moya Huanca, LA, Hart RE. 2016. Changing markets - medicinal plants in the markets of La Paz and El Alto, Bolivia. Journal of Ethnopharmacology. (IF 2015 - 3.055). 
Bussmann RW, Paniagua-Zambrana NY, Randrianarivony T, Andriamihajarivo TH, Rakotoarivony F, Rakotoarivelo $\mathrm{NH}$, Randrianasolo A. 2018. What really is "Harávola" - solving the mystery of the most common basket material in Madagascar. Economic Botany, 72(3): 357-359.

Bussmann RW, Paniagua-Zambrana NY, Romero C, Hart RE. 2018. Astonishing diversity - the medicinal plant markets of Bogotá, Colombia. Journal of Ethnobiology and Ethnomedicine 14(1):43. doi: 10.1186/s13002-018-02418

Bussmann RW, Paniagua-Zambrana NY, Romero C, Hart RE. 2018. No consensus in "traditional" medicine Medicinal plants and their uses in the markets of Bogotá (Colombia), La Paz/El Alto (Bolivia) and Trujillo/Chiclayo (Perú). Indian Journal of Traditional Knowledge 17(3): 494-498.

Bussmann RW, Paniagua-Zambrana NY, Sikharulidze S, Kikvidze Z, Darchidze M, Manvelidze Z, Ekhvaia J, Kikodze D, Tchelidze D, Khutsishvili M, Batsatsashvili K, Hart RE. 2020. Form the sea to the mountains - plant use in Ajara, Samegrelo and Kvemo Svaneti, Sakartvelo (Republic of Georgia), Caucasus. Ethnobotany Research and Applications 20(9). doi: 10.32859/era.20.9.1-34

Bussmann RW, Paniagua-Zambrana NY, Sikharulidze S, Kikvidze Z, Kikodze D, Tchelidze D, Batsatsashvili K, Hart RE. 2021. An ethnobotany of Upper Imereti, Ukana Pshavi, Meshketi and Pankisi gorge, Sakartvelo (Republic of Georgia), Caucasus. Bocconea 29:33-54. doi: 10.732010.7320/Bocc29.001

Bussmann RW, Paniagua-Zambrana NY, Sikharulidze S, Kikvidze Z, Kikodze D, Tchelidze D, Batsatsashvili K, Hart RE. 2018. Unequal brothers - Plant and fungal use in Guria and Racha, Sakartvelo (Republic of Georgia), Caucasus. Indian Journal of Traditional Knowledge 17(1): 7-33.

Bussmann RW, Paniagua-Zambrana NY, Sikharulidze S, Kikvidze Z, Kikodze D, Tchelidze D, Batsatsashvili K, Hart RE. 2017. Plants in the spa - the medicinal plant market of Borjomi, Sakartvelo (Republic of Georgia), Caucasus. Indian Journal of Traditional Knowledge 16(1): 25-34.

Bussmann RW, Paniagua-Zambrana NY, Sikharulidze S, Kikvidze Z, Kikodze D, Tchelidze D, Batsatsashvili K, Hart RE. 2017. Ethnobotany of Samtskhe-Javakheti, Sakartvelo (Republic of Georgia), Caucasus. Indian Journal of Traditional Knowledge 16(1): 7-24.

Bussmann RW, Paniagua-Zambrana NY, Sikharulidze S, Kikvidze Z, Kikodze D, Tchelidze D, Khutsishvili M, Batsatsashvili K, Hart RE, Pieroni A. 2016. Your poison in my pie - the use of Potato (Solanum tuberosum L.) leaves in Sakartvelo, Georgia (Caucasus) and Gollobordo, Eastern Albania. Economic Botany 70(4): 431-437.

Bussmann RW, Paniagua-Zambrana NY, Sikharulidze S, Kikvidze Z, Kikodze D, Tchelidze D, Khutsishvili M, Batsatsashvili K, Hart RE. 2016. Medicinal and food plants of Svaneti and Lechkhumi, Sakartvelo (Republic of Georgia), Caucasus. Medicinal and Aromatic Plants 5:266. doi: 10.4172/2167-0412.1000266.

Bussmann RW, Paniagua-Zambrana NY, Sikharulidze S, Kikvidze Z, Kikodze D, Tchelidze D, Khutsishvili M, Batsatsashvili K, Hart RE. 2016. A comparative ethnobotany of Khevsureti, Samtskhe-Javakheti, Tusheti, Svaneti, and Racha-Lechkhumi, Republic of Georgia (Sakartvelo), Caucasus. Journal of Ehnobiology and Ethnomedicine 12:43. doi: 10.1186/s13002-016-0110-2.

Bussmann RW, Paniagua-Zambrana NY, Sikharulidze S, Kikvidze Z, Kikodze D, Tchelidze D, Batsatsashvili K, Hart RE. 2016. Medicinal and food plants of Svaneti and Lechkhumi, Sakartvelo (Republic of Georgia), Caucasus. Medicinal and Aromatic Plants 5:5.

Bussmann RW, Paniagua-Zambrana NY, Sikharulidze S, Kikvidze Z, Kikodze D, Jinjikhadze T, Shanshiashvili T, Chelidze D, Batsatsashvili K, Bakanidze N. 2014. Wine, Beer, Snuff, Medicine, and Loss of Diversity - Ethnobotanical travels in the Georgian Caucasus. Ethnobotany Research and Applications 12: 237-312.

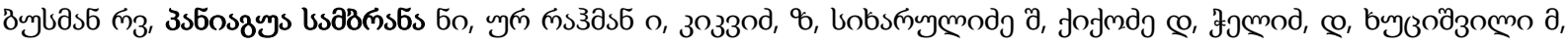

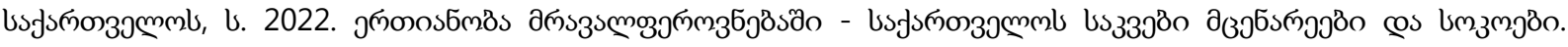
Ethnobotany Research and Applications 23:3. doi: 10.32859/era.23.3.01-69

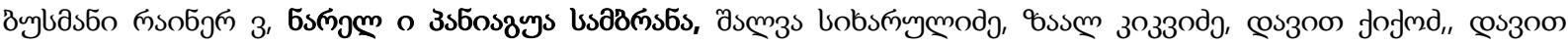

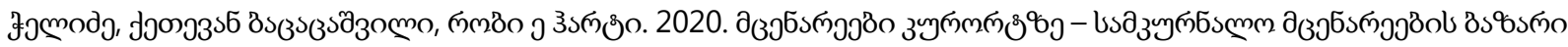
омпхмдðо. Ethnobotany Research and Applications 19(6):1-11. 


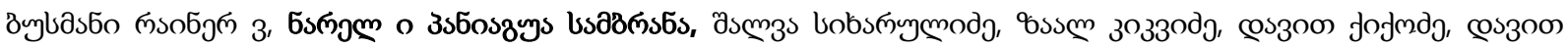

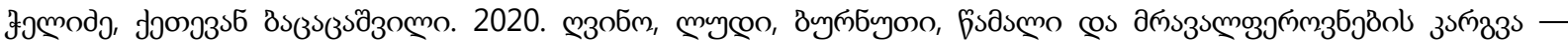

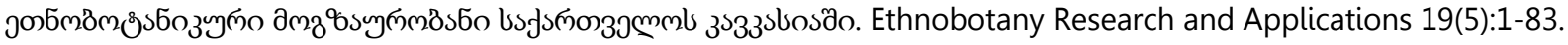

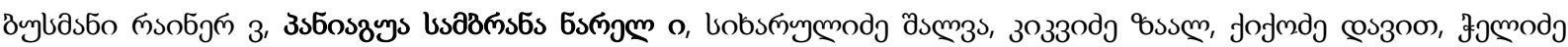

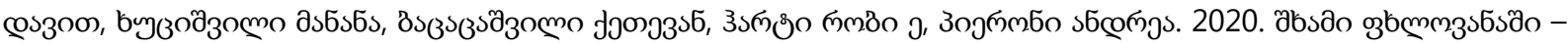

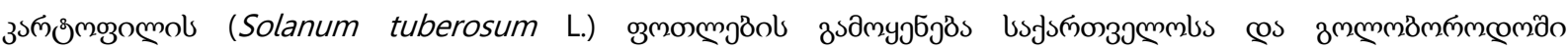

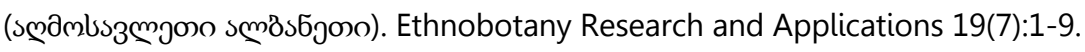

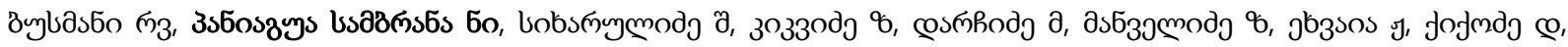

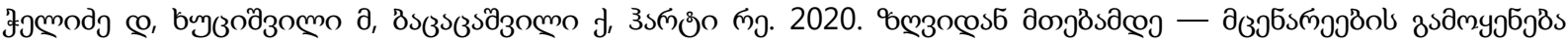

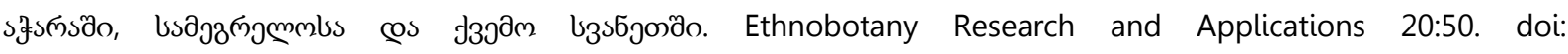
10.32859/era.20.50.1-24

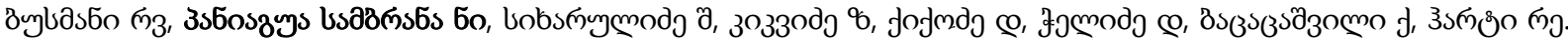

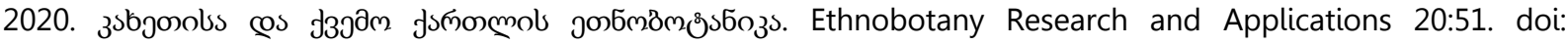
10.32859/era.20.51.1-23

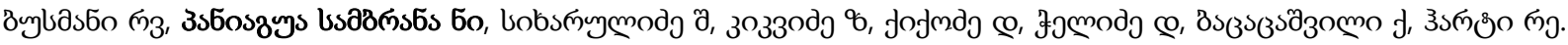

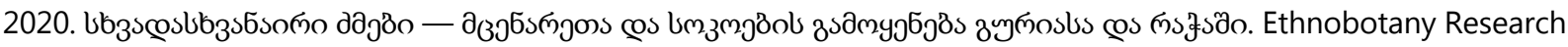
and Applications 20:52. doi: 10.32859/era.20.55.1-34

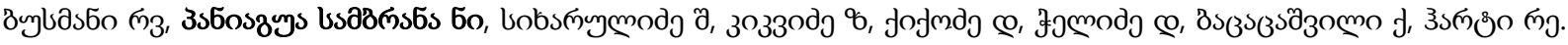

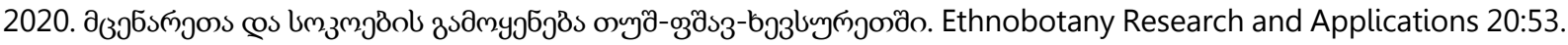
doi: 10.32859/era.20.53.1-30

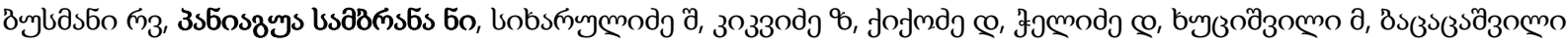

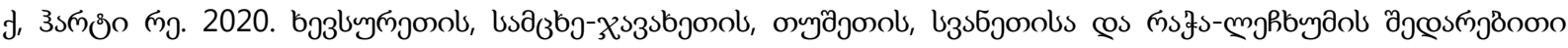

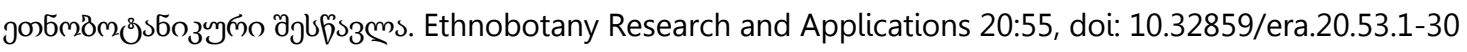

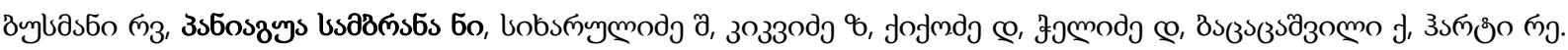

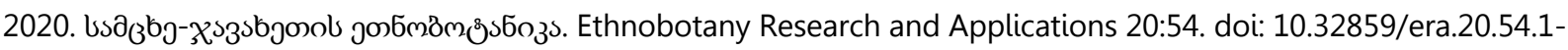
22

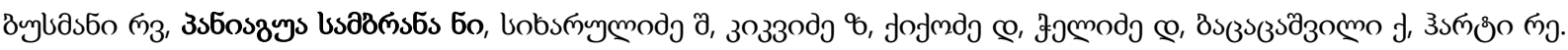

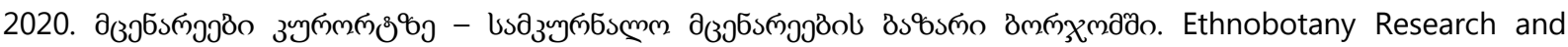
Applications 20:56. doi: 10.32859/era.20.56.1-23

Bussmann RW, Paniagua-Zambrana NY, Tellez C. 2013. Plukenetia carolis-vegae (Euphorbiaceae) - a new useful species from Northern Peru. Economic Botany 67(4): 387-392.

Bussmann RW, Paniagua-Zambrana NY, Ur Rahman I, Kikvidze Z, Sikharulidze S, Kikodze D, Tchelidze D, Khutsishvili M, Batsatsashvili K 2021. Unity in diversity - Food plants and fungi of Sakartvelo (Republic of Georgia), Caucasus. Journal of Ethnobiology and Ethnomedicine 17(72). doi: 10.1186/s13002-021-00490-9

Bussmann RW, Paniagua-Zambrana NY, Wood N, Ole Njapit S, Ole Njapit JN, Ene Osoi GE, Kasoe SP. 2018. Knowledge loss and change between 2002 and 2017 - a revisit of plant-use of the Maasai of Sekenani Valley, Maasai Mara, Kenya. Economic Botany 72(2): 207-216.

Bussmann RW, Paniagua-Zambrana NY. 2012. Axinaea ninakurorum Bussmann, Paniagua - a new species from the Northern Peruvian Merianeae hotspot. Arnaldoa 19(1).

Bussmann RW, Paniagua-Zambrana NY. 2013. Facing global markets - usage changes in Western Amazonian plants: the example of Euterpe precatoria Mart. and E. oleracea Mart. Acta Societatis Botanicorum Poloniae 1(4): 257261.

Bussmann RW, Paniagua-Zambrana NY. 2014. Special Issue: Palms and Communities in the Andes and Amazon of Bolivia and Peru. Ethnobotany Research and Applications 13(1).

Bussmann RW, Paniagua-Zambrana NY. 2014. Traditional knowledge of biodiversity - plant use of the Chácobo in Bolivia. Gaia Scientia. Volume Especial Populações Tradicionais 12: 93-109. 
Bussmann, R.W, Paniagua-Zambrana NY, Sikharulidze S, Kikvidze Z, Kikodze, D, Tchelidze, D, Khutsishvili M, Batsatsashvili K, Hart, R.E. 2020. An ethbobotany iof Kahkheti and Kvemo Kartli, Sakartvelo (Republic of Georgia), Caucasus. Ethnobotany Research and Applications 19(35). doi: 10.32859/era.19.47.1-28.

Cámara Leret R, Paniagua-Zambrana NY, Svenning JC, Balslev H, Macía MJ. 2014. Geospatial patterns in traditional knowledge serve in assessing intellectual property rights and benefit-sharing in northwest South America. Journal of Ethnopharmacology 158 (A): 58-65.

Cámara Leret, R, N. Paniagua Zambrana, H. Balslev and M. J. Macía. 2014. Ethnobotanical knowledge is vastly underdocumented in northwestern South America. PLOS ONE 9(1): e85794.

Cámara-Leret, R, Paniagua-Zambrana NY, Balslev H, Barfod A, Copete JC, Macía MJ. 2014. Ecological community traits and traditional knowledge shape palm ecosystem services in northwestern South America. Forest Ecology and Management 334:28-42.

Castañeda R, Gutiérrez H, Aponte H, Ocampo IZ, Bussmann RW, Paniagua-Zambrana NY. 2021. The trade of wild medicinal plants in the andean district of Lircay, Huancavelica, Perú: A comparative study. Ethnobotany Research and Applications 21(22):1-32. doi: 10.32859/era.21.22.1-32

da Costa Ferreira E, Paiva de Lucena RF, Bussmann RW, Paniagua-Zambrana NY, Dias da Cruz D. 2021. Temporal assessment of the medicinal plant trade in public markets of the state of Paraíba, northeastern Brazil. Journal of Ethnobiology and Ethnomedicine 17:70.. doi: 10.1186/s13002-021-00496-3

Draper FC, Costa FRC, Arellano G, et al. 2021. Amazon tree dominance across forest strata. Nature Ecology and Evolution 5: 757-767. doi: 10.1038/s41559-021-01418-y

Fuentes A, Araujo-M A, Cabrera H, Canqui F, Cayola L, Maldonado C, Paniagua-Zambrana NY. 2005. Estructura, composición y variabilidad de los bosques secos en un sector del valle del río Tuichi, ANMI Madidi, La Paz (Bolivia). Ecología y Conservación Ambiental 15.

Girardin CA, Farfan-Rios W, Garcia K, Feeley KJ, Jørgensen PM, Murakami AA, Cayola Pérez L, Seidel R, PaniaguaZambrana NY, Fuentes Claros AF. 2013. Spatial patterns of above-ground structure, biomass and composition in a network of six Andean elevation transects. Plant Ecology \& Diversity 7:1-11.

Jørgensen PM, Macía MC, Fuentes A, Kessler M, Paniagua-Zambrana NY, Seidel R, Maldonado C, Araujo-M A, Cayola L, Consiglio T, Killeen T, Cabrera WH, Bascopé F, De la Quintana D, Miranda T, Cardona-Peña V, Canqui F. 2005. Lista anotada de las especies vasculares registradas en la región de Madidi. Ecología en Bolivia 40(3): 70-169.

Kupradze I, Jorjadze A, Arabidze A, Beltadze T, Batsatsashvlli K, Paniagua-Zambrana NY, Bussmann RW. 2015. Ethnobiological study of Svaneti Fungi and Lichens: History of research, diversity, local names and traditional use. American Journal of environmental Protection 4(3-1): 101-110.

Macía' MJ, Armesilla J, Cámara-Leret R, Paniagua-Zambrana NY, Villalba S, Balslev H, Manuel Pardo-de-Santayana M. 2011. Palm Uses in North-western South America: a Quantitative Review. The Botanical Review 77(4): 462-570.

Maldonado C, Paniagua-Zambrana NY, Bussmann RW, Zenteno-Ruiz FS, Fuentes AF. 2020. La importancia de las plantas medicinales, su taxonomía y la búsqueda de la cura a la enfermedad que causa el coronavirus (COVID-19). The importance of medicinal plants, their taxonomy and the search for a cure for the disease caused by the coronavirus (COVID-19). Ecología en Bolivia 55(1):1-5.

Myers JA, Chase JM, Jimenez I, Jørgensen PM, Araujo- Murakami A, Paniagua-Zambrana NY, Seidel R. 2013. Betadiversity in temperate and tropical forests reflects dissimilar mechanisms of community assembly. Ecology Letters 16(2): 151-157.

Paniagua-Zambrana NY, Bussmann RW, Hart RE, Moya Huanca AL, Ortiz Soria G, Ortiz Vaca M, Ortiz Álvarez D, Soria Morán J, Soria Morán M, Chávez S, Chávez Moreno B, Chávez Moreno G, Roca O, Siripi E. 2018. Who should conduct ethnobotanical studies? Effects of different interviewers in the case of the Chacobo Ethnobotany project, Beni, Bolivia. Journal of Ethnobiology and Ethnomedicine, 14(1):9. doi: 10.1186/s13002-018-0210-2

Paniagua-Zambrana NY, Bussmann RW, Hart RE, Moya Huanca AL, Ortiz Soria G, Ortiz Vaca M, Ortiz Álvarez D, Soria Morán J, Soria Morán M, Chávez S, Chávez Moreno B, Chávez Moreno G, Roca O, Siripi E. 2018. Research methods leading to a perception of knowledge loss - one century of plant use documentation among the Chacobo in Bolivia. Economic Botany 72(1):81-93. 
Paniagua-Zambrana NY, Bussmann RW, Hart RE, Moya Huanca AL, Ortiz Soria G, Ortiz Vaca M, Ortiz Álvarez D, Soria Morán J, Soria Morán M, Chávez S, Chávez Moreno B, Chávez Moreno G, Roca O, Siripi E. 2017. Traditional knowledge hiding in plain sight - twenty-first century ethnobotany of the Chacobo in Beni, Bolivia. Journal of Ethnobiology and Ethnomedicine 13(1). doi: 10.1186/s13002-017-0179-2

Paniagua-Zambrana NY, Bussmann RW, Hart RE, Moya Huanca LA, Ortiz Soria G, Ortiz Vaca M, Ortiz Álvarez D, Soria Morán J, Soria Morán M, Chávez S, Chávez Moreno B, Chávez Moreno G, Roca O, Siripi E. 2018. To list or not to list? The value and detriment of freelisting in ethnobotanical studies. Nature Plants 4(4):201-204.

Paniagua-Zambrana NY, Bussmann RW, Macía MJ. 2017. The socioeconomic context of the use of Euterpe precatoria Mart. and E. oleracea Mart. in Bolivia and Peru. Journal of Ethnobiology and Ethnomedicine 13(32). doi: 10.1186/s13002-017-0160-0

Paniagua-Zambrana NY, Bussmann RW. 2018. La Etnobotánica de los Chácobo en el Siglo XXI. Ethnobotany Research and Applications 16(2): 1-149. doi: 10.32859/era.16.2.001-149

Paniagua-Zambrana NY, Bussmann RW., Tellez, C, Vega, C. 2018. La Etnobotánica de los Chácobo en el Siglo XX. Ethnobotany Research and Applications 16(1):1-393. doi: 10.32859/era.16.1.001-393

Paniagua-Zambrana NY, Byg A, Svenning JC, Moraes, M, Grandez C, Balslev H. 2007. Diversity of palm uses in the western Amazon. Biodiversity and Conservation (16): 2771-2787.

Paniagua-Zambrana NY, Cámara Leret R, Bussmann RW, Macía MJ. 2014. The influence of socioeconomic factors on traditional knowledge: a cross scale comparison of palm use in northwestern South America. Ecology and Society 19(4): 9.

Paniagua-Zambrana NY, Cámara-Leret R, Macía MJ. 2015 Patters of Medicinal use f palms across Northwestern South America. 2015. The Botanical Review 81(4): 317-415.

Paniagua-Zambrana NY, Cámara-Leret R, Macía MJ. 2016. Understanding transmission of traditional knowledge across north-western South America: a cross-cultural study in palms (Arecaceae). Botanical Journal of Linnean Society 182(2):480-504. doi: 10.1111/boj.12418

Paniagua-Zambrana NY, Macía MJ, Cámara-Leret R. 2010. Protocolo para la toma de datos etnobotánicos de palmeras y variables socioeconómicas en comunidades rurales. Ecología en Bolivia 45(3): 44-68.

Paniagua-Zambrana NY, Maldonado C, Chumacero C. 2003. Mapa de Vegetación y uso de la tierra de la Estación Biológica Tunquini. Ecología en Bolivia 38 (1): 15.26 pp.

Paniagua-Zambrana NY. 2005. Riqueza y uso de las palmas en la región del Madidi, noroeste del departamento de La Paz (Bolivia). Ecología en Bolivia 40(3).

Paniagua-Zambrana NY., Moraes M. 2009. Hacia el manejo del motacú (Attalea phalerata, Arecaceae) bajo diferente tipo de cosecha (Riberalta, depto. Beni, NE Bolivia): Estructura y densidad poblacional. Revista GAB 4:17-23.

Pieroni A, Vandebroek I, Prakofjewa J, Bussmann RW, Paniagua-Zambrana NY, Maroyi A, Torri L, Zocchi DM, Dam ATK, Khan SM, Ahmad H, Yeşil Y, Huish R, Pardo-de-Santayana M, Mocan A, Hu X, Boscolo O, Sõukand R 2020. Taming the pandemic? The importance of homemade plant-based foods and beverages as community responses to COVID-19. Journal of Ethnobiology and Ethnomedicine 16:75. doi: 10.1186/s13002-020-00426-9

Preston D, Fairbain J, Paniagua-Zambrana NY, Mass G. 2003. Grazing and environmental change on the Tarija Altiplano, Bolivia. Mountain Research and development 23 (2): 141-148.

Vandebroek I, Pieroni A, Stepp J, Hanazaki N, Ladio A, Alves RR, Picking D, Delgoda R, Maroyi A, van Andel T, Quave C, Paniagua-Zambrana NY, Bussmann RW, Odonne G, Abbasi A, Albuquerque U, Baker J, Kutz S, Timsina S, Shigeta M, Oliveira T, Hurrell J, Arenas P, Puentes J, Hugé J, Yeşil Y, Jean Pierre L, Olango TM, Dahdouh-Guebas 2020. Reshaping the future of ethnobiology research after the Covid-19 pandemic. Nature Plants 6: 723-730.. doi: 10.1038/s41477-020-0691-6

Zenderland J, Hart R, Bussmann RW, Paniagua-Zambrana NY, Sikharulidze S, Kikvidze Z, Kikodze, D, Tchelidze, D, Khutsishvili M, Batsatsashvili K 2019. The Use of "Use Value": Quantifying Importance in Ethnobotany. Economic Botany 73: 293-303. 


\section{BOOK CHAPTERS and PROCEEDINGS}

Abbasi AM, Bussmann RW, Paniagua-Zambrana NY. 2021. Pyrus pashia Ham. ex D. Don In: Kunwar RW, Sher H, Bussmann RW. Ethnobotany of the Himalayas. Springer, Cham. doi: 10.1007/978-3-030-57408-6_200, pp. 1619-1632.

Abbasi AM, Bussmann, RTW, Paniagua-Zambrana NY. 2021. Astragalus candolleanus Royle ex Benth., Astragalus chlorostachys Lindl., Astragalus hendersonii Baker, Astragalus peduncularis Royle ex Benth. In: Kunwar RW, Sher H, Bussmann RW. Ethnobotany of the Himalayas. Springer, Cham. doi: 10.1007/978-3-030-57408-6_31, pp. 311-316.

Abbasi AM, Kunwar RM, Bussmann RW, Paniagua-Zambrana NY. 2021. Celtis australis L. In: Kunwar RW, Sher H, Bussmann RW. Ethnobotany of the Himalayas. Springer, Cham. doi: 10.1007/978-3-030-57408-6_53, pp. 509-514.

Acharya RP, Sresta R, Kunwar RM, Jan HA, Hussain W, Bussmann RW, Paniagua-Zambrana NY. 2021. Asparagus racemosus Willd. In: Kunwar RW, Sher H, Bussmann RW. Ethnobotany of the Himalayas. Springer, Cham. doi: 10.1007/978-3-030-57408-6_29, pp. 289-304.

Adhikari K, Kunwar RM, Jan HA, Bussmann RW, Paniagua-Zambrana NY. 2021. Brucea javanica (L.) Merr. (Rhus javanica L). In: Kunwar RW, Sher H, Bussmann RW. Ethnobotany of the Himalayas. Springer, Cham. doi: 10.1007/9783-030-57408-6_205, pp. 415-422.

Adhikari YP, Adhikari DM, Kunwar RM, Bussmann RW, Paniagua-Zambrana NY. 2021. Stephania elegans Hook. f. \& Thomson In: Kunwar RW, Sher H, Bussmann RW. Ethnobotany of the Himalayas. Springer, Cham. doi: 10.1007/9783-030-57408-6_235, pp. 1933-1940.

Adhikari YP, Bhattarai P, Acharya KP, Kunwar RM, Bussmann RW. 2021. Dendrobium nobile Lindl. In: Kunwar RW, Sher H, Bussmann RW. Ethnobotany of the Himalayas. Springer, Cham. doi: 10.1007/978-3-030-57408-6_80, pp. 747752 .

Amatya G, Kunwar RM, Bussmann RW, Paniagua-Zambrana NY. 2021. Debregeasia longifolia (Burm. f.) Wedd. In: Kunwar RW, Sher H, Bussmann RW. Ethnobotany of the Himalayas. Springer, Cham. doi: 10.1007/978-3-030-574086_78, pp. 735-738.

Ansari AS, Thakuri U, Kunwar RM, Bussmann RW, Paniagua-Zambrana NY. 2021. Syzygium cumini (L.) Skeels In: Kunwar RW, Sher H, Bussmann RW. Ethnobotany of the Himalayas. Springer, Cham. doi: 10.1007/978-3-030-574086_239, pp. 1955-1966.

Aryal S, Poudyal BH, Kunwar RM, Bussmann RW, Paniagua-Zambrana NY. 2021. Jurinea dolomitica/ Galushko In: Kunwar RW, Sher H, Bussmann RW. Ethnobotany of the Himalayas. Springer, Cham. doi: 10.1007/978-3-030-574086_135, pp. 1157-1162.

Awasthi B, Kunwar RM, Bussmann RW, Paniagua-Zambrana NY. 2021. Angelica glauca Edgew. In: Kunwar RW, Sher H, Bussmann RW. Ethnobotany of the Himalayas. Springer, Cham. doi: 10.1007/978-3-030-57408-6_23, pp. 239-248.

Balayar R, Kunwar RM, Bussmann RW. 2021. Schleichera oleosa (Lour.) Oken. In: Kunwar RW, Sher H, Bussmann RW. Ethnobotany of the Himalayas. Springer, Cham. doi: 10.1007/978-3-030-57408-6_220, pp. 1813-1818.

Balslev H, Paniagua-Zambrana NY, Moraes M, Grandez C, Byg A. 2007. Local knowledge of tropical rainforest palms in the Pastaza fan in the western Amazon basin. Pp. 41. In: Resúmenes - Simposio Internacional Las Palmeras en el Marco de la Investigación para el Desarrollo en América del Sur, 7-9 Noviembre, Universidad Nacional Mayor de San Marcos-IRD, Lima.

Baral B, Kunwar RM, Bussmann RW, Paniagua-Zambrana NY. 2021. Lobelia pyramidalis Wall. In: Kunwar RW, Sher H, Bussmann RW. Ethnobotany of the Himalayas. Springer, Cham. doi: 10.1007/978-3-030-57408-6_143, pp. 12051210.

Baral K, Kunwar RM, Bussmann RW, Paniagua-Zambrana NY. 2021. Crateva unilocularis Buch.-Ham. In: Kunwar RW, Sher H, Bussmann RW. Ethnobotany of the Himalayas. Springer, Cham. doi: 10.1007/978-3-030-57408-6_67, pp. 629634.

Baral S, Gauli K, Kunwar RM, Ali M, Bussmann RW, Paniagua-Zambrana NY. 2021. Prinsepia utilis Royle In: Kunwar RW, Sher H, Bussmann RW. Ethnobotany of the Himalayas. Springer, Cham. doi: 10.1007/978-3-030-57408-6_197, pp. 1597-1606. 
Basnet R, Kunwar RM, Bussmann RW, Paniagua-Zambrana NY. 2021. Duhaldea cappa (Buch.-Ham. ex D.Don) Pruski \& Anderb., Inula nervosa Wall. ex DC. In: Kunwar RW, Sher H, Bussmann RW. Ethnobotany of the Himalayas. Springer, Cham. doi: 10.1007/978-3-030-57408-6_125, pp. 825-834.

Batsatsashvili K, Kikvidze Z, Khutsishvili M, Maisaia I, Sikharulidze S, Tchelidze D, Paniagua-Zambrana NY, Bussmann RW. 2017. Abies nordmanniana (Steven) Spach. In: Bussmann RW (ed.) Ethnobotany of the Caucasus, Springer International Publishing, Cham. doi: 10.1007/978-3-319-49412-8_20

Batsatsashvili K, Kikvidze Z, Khutsishvili M, Maisaia I, Sikharulidze S, Tchelidze D, Paniagua-Zambrana NY, Bussmann RW. 2017. Agasyllis latifolia (M. Bieb.) Boiss. In: Bussmann RW (ed.) Ethnobotany of the Caucasus, Springer International Publishing, Cham. doi: 10.1007/978-3-319-49412-8_138

Batsatsashvili K, Kikvidze Z, Khutsishvili M, Maisaia I, Sikharulidze S, Tchelidze D, Paniagua-Zambrana NY, Bussmann RW. 2017. Campanula lactiflora M. Bieb. In: Bussmann RW (ed.) Ethnobotany of the Caucasus, Springer International Publishing, Cham. doi: 10.1007/978-3-319-49412-8_33

Batsatsashvili K, Kikvidze Z, Khutsishvili M, Maisaia I, Sikharulidze S, Tchelidze D, Paniagua-Zambrana NY, Bussmann RW. 2017. Chenopodium album L., Chenopodium foliosum L. In: Bussmann RW (ed.) Ethnobotany of the Caucasus, Springer International Publishing, Cham. doi: 10.1007/978-3-319-49412-8_113

Batsatsashvili K, Kikvidze Z, Khutsishvili M, Maisaia I, Sikharulidze S, Tchelidze D, Paniagua-Zambrana NY, Bussmann RW. 2017. Fritillaria collina Adams. In: Bussmann RW (ed.) Ethnobotany of the Caucasus, Springer International Publishing, Cham. doi: 10.1007/978-3-319-49412-8_37

Batsatsashvili K, Kikvidze Z, Khutsishvili M, Maisaia I, Sikharulidze S, Tchelidze D, Paniagua-Zambrana NY, Bussmann RW. 2017. Heracleum asperum M. B. Fl., Heracleum leskovii A. Grossh. In: Bussmann RW (ed.) Ethnobotany of the Caucasus, Springer International Publishing, Cham. doi: 10.1007/978-3-319-49412-8_55

Batsatsashvili K, Kikvidze Z, Khutsishvili M, Maisaia I, Sikharulidze S, Tchelidze D, Paniagua-Zambrana NY, Bussmann RW. 2017. Lathyrus roseus StevenIn: Bussmann RW (ed.) Ethnobotany of the Caucasus, Springer International Publishing, Cham. doi: 10.1007/978-3-319-49412-8_53

Batsatsashvili K, Kikvidze Z, Khutsishvili M, Maisaia I, Sikharulidze S, Tchelidze D, Paniagua-Zambrana NY, Bussmann RW. 2017. Petasites albus (L.) Gaertn, Petasites. fominii Bordz, Petasites hybridus (L.) G. Gaertn., B. Mey. \& Scherb. In: Bussmann RW (ed.) Ethnobotany of the Caucasus, Springer International Publishing, Cham. doi: 10.1007/978-3319-49412-8_57

Batsatsashvili K, Kikvidze Z, Khutsishvili M, Maisaia I, Sikharulidze S, Tchelidze D, Paniagua-Zambrana NY, Bussmann RW. 2017. Picea orientalis (L.) Peterm. In: Bussmann RW (ed.) Ethnobotany of the Caucasus, Springer International Publishing, Cham. doi: 10.1007/978-3-319-49412-8_69

Batsatsashvili K, Kikvidze Z, Khutsishvili M, Maisaia I, Sikharulidze S, Tchelidze D, Paniagua-Zambrana NY, Bussmann RW. 2017. Rapistrum rugosum (L.) All. In: Bussmann RW (ed.) Ethnobotany of the Caucasus, Springer International Publishing, Cham. doi: 10.1007/978-3-319-49412-8_35

Batsatsashvili K, Kikvidze Z, Khutsishvili M, Maisaia I, Sikharulidze S, Tchelidze D, Paniagua-Zambrana NY, Bussmann RW. 2017. Sambucus ebulus L. In: Bussmann RW (ed.) Ethnobotany of the Caucasus, Springer International Publishing, Cham. doi: 10.1007/978-3-319-49412-8_46

Batsatsashvili K, Kikvidze Z, Khutsishvili M, Maisaia I, Sikharulidze S, Tchelidze D, Paniagua-Zambrana NY, Bussmann RW. 2017. Satureja laxiflora C. Koch, Satureja spicigera (K. Koch) Boiss. In: Bussmann RW (ed.) Ethnobotany of the Caucasus, Springer International Publishing, Cham. doi: 10.1007/978-3-319-49412-8_65

Batsatsashvili K, Kikvidze Z, Khutsishvili M, Maisaia I, Sikharulidze S, Tchelidze D, Paniagua-Zambrana NY, Bussmann RW. 2017. Smilax excelsa L. In: Bussmann RW (ed.) Ethnobotany of the Caucasus, Springer International Publishing, Cham. doi: 10.1007/978-3-319-49412-8_98

Batsatsashvili K, Kikvidze Z, Khutsishvili M, Maisaia I, Sikharulidze S, Tchelidze D, Paniagua-Zambrana NY, Bussmann RW. 2017. Staphylea colchica Steven., Staphylea pinnata L. In: Bussmann RW (ed.) Ethnobotany of the Caucasus, Springer International Publishing, Cham. doi: 10.1007/978-3-319-49412-8_121 
Batsatsashvili K, Kikvidze Z, Khutsishvili M, Maisaia I, Sikharulidze S, Tchelidze D, Paniagua-Zambrana NY, Bussmann RW. 2017. Vaccinium arctostaphylos L. In: Bussmann RW (ed.) Ethnobotany of the Caucasus, Springer International Publishing, Cham. doi: 10.1007/978-3-319-49412-8_78

Batsatsashvili K, Mehdiyeva N, Fayvush G, Kikvidze Z, Khutsishvili M, Maisaia I, Sikharulidze S, Tchelidze D, Alizade V, Aleksanyan A, Paniagua Zambrana, N.Y Bussmann RW. 2017. Artemisia annua L., Artemisia fragrans Willd. In: Bussmann RW (ed.) Ethnobotany of the Caucasus, Springer International Publishing, Cham. doi: 10.1007/978-3-31949412-8_127

Batsatsashvili K, Mehdiyeva N, Fayvush G, Kikvidze Z, Khutsishvili M, Maisaia I, Sikharulidze S, Tchelidze D, Aleksanyan A, Alizade V, Paniagua-Zambrana NY, Bussmann RW. 2017. Berberis vulgaris L. In: Bussmann RW (ed.) Ethnobotany of the Caucasus, Springer International Publishing, Cham. doi: 10.1007/978-3-319-49412-8_144

Batsatsashvili K, Mehdiyeva N, Fayvush G, Kikvidze Z, Khutsishvili M, Maisaia I, Sikharulidze S, Tchelidze D, Aleksanyan A, Alizade V, Paniagua-Zambrana NY, Bussmann RW. 2017. Cornus mas L. In: Bussmann RW (ed.) Ethnobotany of the Caucasus, Springer International Publishing, Cham. doi: 10.1007/978-3-319-49412-8_108

Batsatsashvili K, Mehdiyeva N, Fayvush G, Kikvidze Z, Khutsishvili M, Maisaia I, Sikharulidze S, Tchelidze D, Aleksanyan A, Alizade V, Paniagua-Zambrana NY, Bussmann RW. 2017. Corylus avellana L., Corylus colurna L. In: Bussmann RW (ed.) Ethnobotany of the Caucasus, Springer International Publishing, Cham. doi: 10.1007/978-3-319-49412-8_25

Batsatsashvili K, Mehdiyeva N, Fayvush G, Kikvidze Z, Khutsishvili M, Maisaia I, Sikharulidze S, Tchelidze D, Aleksanyan A, Alizade V, Paniagua-Zambrana NY, Bussmann RW. 2017. Crataegus curvisepala Lindm., Crataegus pentagyna Waldst. In: Bussmann RW (ed.) Ethnobotany of the Caucasus, Springer International Publishing, Cham. doi: 10.1007/978-3-319-49412-8_123

Batsatsashvili K, Mehdiyeva N, Fayvush G, Kikvidze Z, Khutsishvili M, Maisaia I, Sikharulidze S, Tchelidze D, Aleksanyan A, Alizade V, Paniagua-Zambrana NY, Bussmann RW. 2017. Echium maculatum L. In: Bussmann RW (ed.) Ethnobotany of the Caucasus, Springer International Publishing, Cham. doi: 10.1007/978-3-319-49412-8_29

Batsatsashvili K, Mehdiyeva N, Fayvush G, Kikvidze Z, Khutsishvili M, Maisaia I, Sikharulidze S, Tchelidze D, Aleksanyan A, Alizade V, Paniagua-Zambrana NY, Bussmann RW. 2017. Fagus orientalis Lipsky. In: Bussmann RW (ed.) Ethnobotany of the Caucasus, Springer International Publishing, Cham. doi: 10.1007/978-3-319-49412-8_70

Batsatsashvili K, Mehdiyeva N, Fayvush G, Kikvidze Z, Khutsishvili M, Maisaia I, Sikharulidze S, Tchelidze D, Aleksanyan A, Alizade V, Paniagua-Zambrana NY, Bussmann RW. 2017. Falcaria vulgaris Bernh. In: Bussmann RW (ed.) Ethnobotany of the Caucasus, Springer International Publishing, Cham. doi: 10.1007/978-3-319-49412-8_36

Batsatsashvili K, Mehdiyeva N, Fayvush G, Kikvidze Z, Khutsishvili M, Maisaia I, Sikharulidze S, Tchelidze D, Alizade V, Aleksanyan A, Paniagua-Zambrana NY, Bussmann RW. 2017. Juglans regia L. In: Bussmann RW (ed.) Ethnobotany of the Caucasus, Springer International Publishing, Cham. doi: 10.1007/978-3-319-49412-8_22

Batsatsashvili K, Mehdiyeva N, Fayvush G, Kikvidze Z, Khutsishvili M, Maisaia I, Sikharulidze S, Tchelidze D, Alizade V, Aleksanyan A, Paniagua-Zambrana NY, Bussmann RW. 2017. Malva neglectaWallr., Malva sylvestris L. In: Bussmann RW (ed.) Ethnobotany of the Caucasus, Springer International Publishing, Cham. doi: 10.1007/978-3-319-494128_115

Batsatsashvili K, Mehdiyeva N, Fayvush G, Kikvidze Z, Khutsishvili M, Maisaia I, Sikharulidze S, Tchelidze D, Alizade V, Aleksanyan A, Paniagua-Zambrana NY, Bussmann RW. 2017. Mentha aquatica L., Mentha longifolia L., Mentha pulegium L. In: Bussmann RW (ed.) Ethnobotany of the Caucasus, Springer International Publishing, Cham. doi: 10.1007/978-3-319-49412-8_141

Batsatsashvili K, Mehdiyeva N, Fayvush G, Kikvidze Z, Khutsishvili M, Maisaia I, Sikharulidze S, Tchelidze D, Alizade V, Aleksanyan A, Paniagua-Zambrana NY, Bussmann RW. 2017. Mespilus germanica L.In: Bussmann RW (ed.) Ethnobotany of the Caucasus, Springer International Publishing, Cham. doi: 10.1007/978-3-319-49412-8_93

Batsatsashvili K, Mehdiyeva N, Fayvush G, Kikvidze Z, Khutsishvili M, Maisaia I, Sikharulidze S, Tchelidze D, Aleksanyan A, Alizade V, Paniagua-Zambrana NY, Bussmann RW. 2017. Pinus kochiana Klotzsch ex K. Koch. In: Bussmann RW (ed.) Ethnobotany of the Caucasus, Springer International Publishing, Cham. doi: 10.1007/978-3-319-49412-8_51

Batsatsashvili K, Mehdiyeva N, Fayvush G, Kikvidze Z, Khutsishvili M, Maisaia I, Sikharulidze S, Tchelidze D, Aleksanyan A, Alizade V, Paniagua-Zambrana NY, Bussmann RW. 2017. Polygonum alpinum All., Polygonum aviculare L., 
Polygonum carneum C. Koch, Polygonum hydropiper L. In: Bussmann RW (ed.) Ethnobotany of the Caucasus, Springer International Publishing, Cham. doi: 10.1007/978-3-319-49412-8_80

Batsatsashvili K, Mehdiyeva N, Fayvush G, Kikvidze Z, Khutsishvili M, Maisaia I, Sikharulidze S, Tchelidze D, Aleksanyan A, Alizade V, Paniagua-Zambrana NY, Bussmann RW. 2017. Portulaca oleracea L. In: Bussmann RW (ed.) Ethnobotany of the Caucasus, Springer International Publishing, Cham. doi: 10.1007/978-3-319-49412-8_119

Batsatsashvili K, Mehdiyeva N, Fayvush G, Kikvidze Z, Khutsishvili M, Maisaia I, Sikharulidze S, Tchelidze D, Aleksanyan A, Alizade V, Paniagua Zambrana, N.Y Bussmann RW. 2017. Prunus divaricata Ledeb. In: Bussmann RW (ed.) Ethnobotany of the Caucasus, Springer International Publishing, Cham. doi: 10.1007/978-3-319-49412-8_75

Batsatsashvili K, Mehdiyeva N, Fayvush G, Kikvidze Z, Khutsishvili M, Maisaia I, Sikharulidze S, Tchelidze D, Aleksanyan A, Alizade V, Paniagua-Zambrana NY, Bussmann RW. 2017. Prunus spinosa L. In: Bussmann RW (ed.) Ethnobotany of the Caucasus, Springer International Publishing, Cham. doi: 10.1007/978-3-319-49412-8_73

Batsatsashvili K, Mehdiyeva N, Fayvush G, Kikvidze Z, Khutsishvili M, Maisaia I, Sikharulidze S, Tchelidze D, Aleksanyan A, Alizade V, Paniagua-Zambrana NY, Bussmann RW. 2017. Pyrus caucasica Fed. In: Bussmann RW (ed.) Ethnobotany of the Caucasus, Springer International Publishing, Cham. doi: 10.1007/978-3-319-49412-8_63

Batsatsashvili K, Mehdiyeva N, Fayvush G, Kikvidze Z, Khutsishvili M, Maisaia I, Sikharulidze S, Tchelidze D, Aleksanyan A, Alizade V, Paniagua-Zambrana NY, Bussmann RW. 2017. Quercus iberica Steven ex M. Bieb. In: Bussmann RW (ed.) Ethnobotany of the Caucasus, Springer International Publishing, Cham. doi: 10.1007/978-3-319-49412-8_54

Batsatsashvili K, Mehdiyeva N, Fayvush G, Kikvidze Z, Khutsishvili M, Maisaia I, Sikharulidze S, Tchelidze D, Aleksanyan A, Alizade V, Paniagua-Zambrana NY, Bussmann RW. 2017. Ribes alpinum L., Ribes orientale Desf., Ribes petraeum Wulfen, Ribes uva-crispa L. In: Bussmann RW (ed.) Ethnobotany of the Caucasus, Springer International Publishing, Cham. doi: 10.1007/978-3-319-49412-8_59

Batsatsashvili K, Mehdiyeva N, Fayvush G, Kikvidze Z, Khutsishvili M, Maisaia I, Sikharulidze S, Tchelidze D, Aleksanyan A, Alizade V, Paniagua-Zambrana NY, Bussmann RW. 2017. Rumex acetosa L., Rumex acetosella L., Rumex confertus Willd., Rumex conglomeratus Murray, Rumex crispus L., Rumex tuberosus L. In: Bussmann RW (ed.) Ethnobotany of the Caucasus, Springer International Publishing, Cham. doi: 10.1007/978-3-319-49412-8_131

Batsatsashvili K, Mehdiyeva N, Fayvush G, Kikvidze Z, Khutsishvili M, Maisaia I, Sikharulidze S, Tchelidze D, Aleksanyan A, Alizade V, Paniagua-Zambrana NY, Bussmann RW. 2017. Sempervivum caucasicum Rupr., Sempervivum annae Gurgenidze. In: Bussmann RW (ed.) Ethnobotany of the Caucasus, Springer International Publishing, Cham. doi: 10.1007/978-3-319-49412-8_97

Batsatsashvili K, Mehdiyeva N, Fayvush G, Kikvidze Z, Khutsishvili M, Maisaia I, Sikharulidze S, Tchelidze D, Aleksanyan A, Alizade V, Paniagua-Zambrana NY, Bussmann RW. 2017. Sorbus aucuparia L., Sorbus torminalis (L.) Crantz. In: Bussmann RW (ed.) Ethnobotany of the Caucasus, Springer International Publishing, Cham. doi: 10.1007/978-3-31949412-8_133

Batsatsashvili K, Mehdiyeva N, Fayvush G, Kikvidze Z, Khutsishvili M, Maisaia I, Sikharulidze S, Tchelidze D, Aleksanyan A, Alizade V, Paniagua-Zambrana NY, Bussmann RW. 2017. Stellaria media (L.) Vill. In: Bussmann RW (ed.) Ethnobotany of the Caucasus, Springer International Publishing, Cham. doi: 10.1007/978-3-319-49412-8_45

Batsatsashvili K, Mehdiyeva N, Fayvush G, Kikvidze Z, Khutsishvili M, Maisaia I, Sikharulidze S, Tchelidze D, Aleksanyan A, Alizade V, Paniagua-Zambrana NY, Bussmann RW. 2017. Symphytum caucasicum M. Bieb. In: Bussmann RW (ed.) Ethnobotany of the Caucasus, Springer International Publishing, Cham. doi: 10.1007/978-3-319-49412-8_61

Batsatsashvili K, Mehdiyeva N, Fayvush G, Kikvidze Z, Khutsishvili M, Maisaia I, Sikharulidze S, Tchelidze D, Aleksanyan A, Alizade V, Paniagua-Zambrana NY, Bussmann RW. 2017. Thymus caucasicus Willd. ex Benth., Thymus collinus M. Bieb., Thymus kotschyanus Boiss. \& Hohen. In: Bussmann RW (ed.) Ethnobotany of the Caucasus, Springer International Publishing, Cham. doi: 10.1007/978-3-319-49412-8_101

Batsatsashvili K, Mehdiyeva N, Fayvush G, Kikvidze Z, Khutsishvili M, Maisaia I, Sikharulidze S, Tchelidze D, Aleksanyan A, Alizade V, Paniagua-Zambrana NY, Bussmann RW. 2017. Urtica dioica L. In: Bussmann RW (ed.) Ethnobotany of the Caucasus, Springer International Publishing, Cham. doi: 10.1007/978-3-319-49412-8_140

Batsatsashvili K, Mehdiyeva N, Kikvidze Z, Khutsishvili M, Maisaia I, Sikharulidze S, Tchelidze D, Alizade V, PaniaguaZambrana NY, Bussmann RW. 2017. Amaranthus retroflexus L. In: Bussmann RW (ed.) Ethnobotany of the Caucasus, Springer International Publishing, Cham. doi: 10.1007/978-3-319-49412-8_137 
Batsatsashvili K, Mehdiyeva N, Kikvidze Z, Khutsishvili M, Maisaia I, Sikharulidze S, Tchelidze D, Alizade V, PaniaguaZambrana NY, Bussmann RW. 2017. Betula pubescens var. litwinowii (Doluch.) Ashburner \& Mc. All. In: Bussmann RW (ed.) Ethnobotany of the Caucasus, Springer International Publishing, Cham. doi: 10.1007/978-3-319-494128_147

Batsatsashvili K, Mehdiyeva N, Kikvidze Z, Khutsishvili M, Maisaia I, Sikharulidze S, Tchelidze D, Alizade V, PaniaguaZambrana NY, Bussmann RW. 2017. Carpinus caucasica Grossh. In: Bussmann RW (ed.) Ethnobotany of the Caucasus, Springer International Publishing, Cham. doi: 10.1007/978-3-319-49412-8_71

Batsatsashvili K, Mehdiyeva N, Kikvidze Z, Khutsishvili M, Maisaia I, Sikharulidze S, Tchelidze D, Alizade V, PaniaguaZambrana NY, Bussmann RW. 2017. Celtis caucasica Willd. In: Bussmann RW (ed.) Ethnobotany of the Caucasus, Springer International Publishing, Cham. doi: 10.1007/978-3-319-49412-8_148

Batsatsashvili K, Mehdiyeva N, Kikvidze Z, Khutsishvili M, Maisaia I, Sikharulidze S, Tchelidze D, Alizade V, PaniaguaZambrana NY, Bussmann RW. 2017. Chelidonium majus L. In: Bussmann RW (ed.) Ethnobotany of the Caucasus, Springer International Publishing, Cham. doi: 10.1007/978-3-319-49412-8_88

Batsatsashvili K, Mehdiyeva N, Kikvidze Z, Khutsishvili M, Maisaia I, Sikharulidze S, Tchelidze D, Alizade V, PaniaguaZambrana NY, Bussmann RW. 2017. Cirsium arvense (L.) Scop. In: Bussmann RW (ed.) Ethnobotany of the Caucasus, Springer International Publishing, Cham. doi: 10.1007/978-3-319-49412-8_62

Batsatsashvili K, Mehdiyeva N, Kikvidze Z, Khutsishvili M, Maisaia I, Sikharulidze S, Tchelidze D, Alizade V, PaniaguaZambrana NY, Bussmann RW. 2017. Dryopteris filix-mas (L.) Schott. In: Bussmann RW (ed.) Ethnobotany of the Caucasus, Springer International Publishing, Cham. doi: 10.1007/978-3-319-49412-8_87

Batsatsashvili K, Mehdiyeva N, Kikvidze Z, Khutsishvili M, Maisaia I, Sikharulidze S, Tchelidze D, Alizade V, PaniaguaZambrana NY, Bussmann RW. 2017. Gentiana septemfida Pall. In: Bussmann RW (ed.) Ethnobotany of the Caucasus, Springer International Publishing, Cham. doi: 10.1007/978-3-319-49412-8_84

Batsatsashvili K, Mehdiyeva N, Kikvidze Z, Khutsishvili M, Maisaia I, Sikharulidze S, Tchelidze D, Alizade V, PaniaguaZambrana NY, Bussmann RW. 2017. Inula helenium L. In: Bussmann RW (ed.) Ethnobotany of the Caucasus, Springer International Publishing, Cham. doi: 10.1007/978-3-319-49412-8_89

Batsatsashvili K, Mehdiyeva N, Kikvidze Z, Khutsishvili M, Maisaia I, Sikharulidze S, Tchelidze D, Alizade V, PaniaguaZambrana NY, Bussmann RW. 2017. Juniperus communis L., Juniperus sabina L. In: Bussmann RW (ed.) Ethnobotany of the Caucasus, Springer International Publishing, Cham. doi: 10.1007/978-3-319-49412-8_129

Batsatsashvili K, Mehdiyeva N, Kikvidze Z, Khutsishvili M, Maisaia I, Sikharulidze S, Tchelidze D, Alizade V, PaniaguaZambrana NY, Bussmann RW. 2017. Lamium album L. In: Bussmann RW (ed.) Ethnobotany of the Caucasus, Springer International Publishing, Cham. doi: 10.1007/978-3-319-49412-8_120

Batsatsashvili K, Mehdiyeva N, Kikvidze Z, Khutsishvili M, Maisaia I, Sikharulidze S, Tchelidze D, Alizade V, PaniaguaZambrana NY, Bussmann RW. 2017. Matteuccia struthiopteris (L.) Todd. In: Bussmann RW (ed.) Ethnobotany of the Caucasus, Springer International Publishing, Cham. doi: 10.1007/978-3-319-49412-8_60

Batsatsashvili K, Mehdiyeva N, Kikvidze Z, Khutsishvili M, Maisaia I, Sikharulidze S, Tchelidze D, Alizade V, PaniaguaZambrana NY, Bussmann RW. 2017. Oberna lacera (Steven) Ikonn., Oberna wallichiana (Klotzsch) Ikonn. In: Bussmann RW (ed.) Ethnobotany of the Caucasus, Springer International Publishing, Cham. doi: 10.1007/978-3-31949412-8_39

Batsatsashvili K, Mehdiyeva N, Kikvidze Z, Khutsishvili M, Maisaia I, Sikharulidze S, Tchelidze D, Alizade V, PaniaguaZambrana NY, Bussmann RW. 2017. Rhododendron caucasicum Pall. In: Bussmann RW (ed.) Ethnobotany of the Caucasus, Springer International Publishing, Cham. doi: 10.1007/978-3-319-49412-8_91

Batsatsashvili K, Mehdiyeva N, Kikvidze Z, Khutsishvili M, Maisaia I, Sikharulidze S, Tchelidze D, Alizade V, PaniaguaZambrana NY, Bussmann RW. 2017. Rosa canina L., Rosa iberica Stev., Rosa villosa L. In: Bussmann RW (ed.) Ethnobotany of the Caucasus, Springer International Publishing, Cham. doi: 10.1007/978-3-319-49412-8_64

Batsatsashvili K, Mehdiyeva N, Kikvidze Z, Khutsishvili M, Maisaia I, Sikharulidze S, Tchelidze D, Alizade V, PaniaguaZambrana NY, Bussmann RW. 2017. Rubia tinctorium L. In: Bussmann RW (ed.) Ethnobotany of the Caucasus, Springer International Publishing, Cham. doi: 10.1007/978-3-319-49412-8_47 
Batsatsashvili K, Mehdiyeva N, Kikvidze Z, Khutsishvili M, Maisaia I, Sikharulidze S, Tchelidze D, Alizade V, PaniaguaZambrana NY, Bussmann RW. 2017. Sambucus nigra L. In: Bussmann RW (ed.) Ethnobotany of the Caucasus, Springer International Publishing, Cham. doi: 10.1007/978-3-319-49412-8_27

Batsatsashvili K, Mehdiyeva N, Kikvidze Z, Khutsishvili M, Maisaia I, Sikharulidze S, Tchelidze D, Alizade V, PaniaguaZambrana NY, Bussmann RW. 2017. Sedum caucasicum (Grossh.) Boriss., Sedum spurium M. Bieb. In: Bussmann RW (ed.) Ethnobotany of the Caucasus, Springer International Publishing, Cham. doi: 10.1007/978-3-319-494128_128

Batsatsashvili K, Mehdiyeva N, Kikvidze Z, Khutsishvili M, Maisaia I, Sikharulidze S, Tchelidze D, Alizade V, PaniaguaZambrana NY, Bussmann, R.W.2017). Seseli transcaucasicum Pimenov \& Sdobnina. In: Bussmann RW (ed.) Ethnobotany of the Caucasus, Springer International Publishing, Cham. doi: 10.1007/978-3-319-49412-8_116

Batsatsashvili K, Mehdiyeva N, Kikvidze Z, Khutsishvili M, Maisaia I, Sikharulidze S, Tchelidze D, Alizade V, PaniaguaZambrana NY, Bussmann RW. 2017. Solanum nigrum L. In: Bussmann RW (ed.) Ethnobotany of the Caucasus, Springer International Publishing, Cham. doi: 10.1007/978-3-319-49412-8_117

Batsatsashvili K, Mehdiyeva N, Kikvidze Z, Khutsishvili M, Maisaia I, Sikharulidze S, Tchelidze D, Alizade V, PaniaguaZambrana NY, Bussmann RW. 2017. Tilia begonïfolia Steven. In: Bussmann RW (ed.) Ethnobotany of the Caucasus, Springer International Publishing, Cham. doi: 10.1007/978-3-319-49412-8_38

Batsatsashvili K, Mehdiyeva N, Kikvidze Z, Khutsishvili M, Maisaia I, Sikharulidze S, Tchelidze D, Alizade V, PaniaguaZambrana NY, Bussmann RW. 2017. Viburnum lantana L. In: Bussmann RW (ed.) Ethnobotany of the Caucasus, Springer International Publishing, Cham. doi: 10.1007/978-3-319-49412-8_43

Batsatsashvili K, Mehdiyeva N, Kikvidze Z, Khutsishvili M, Maisaia I, Sikharulidze S, Tchelidze D, Alizade V, PaniaguaZambrana NY, Bussmann RW. 2017. Ziziphora serpyllacea M. Bieb. In: Bussmann RW (ed.) Ethnobotany of the Caucasus, Springer International Publishing, Cham. doi: 10.1007/978-3-319-49412-8_72

Beck S, Paniagua-Zambrana NY, Paz C. 1999. Potencialidades de los recursos fitogenéticos nativos de Bolivia. In Memorias de la Segunda Reunión Boliviana sobre Recursos Fitogenéticos de Cultivos Nativos Cochabamba, Bolivia.

Beck S, Paniagua-Zambrana NY, Yevara M, Liberman M. 2001. La vegetación y uso de la tierra en el altiplano y los valles en el oeste del departamento de Tarija, Bolivia. In Historia, Ambiente y Sociedad en Tarija, Bolivia. Beck, S, N. Paniagua \& D. Preston (eds.). Instituto de Ecología, UMSA/Escuela de Geografía/Universidad de Leeds. Editorial Grafica Latina. La Paz, Bolivia.

Beck S, Paniagua-Zambrana NY, Yevara M. 1999. El cambio del medio ambiente-Un efecto natural o humano? Estudio multidisciplinario en la región sud-central de los Andes. In: Memorias del IV Congreso Latinoamericano de Ecología. Universidad de San Agustín. Arequipa, Perú.

Beck S, Paniagua-Zambrana NY, Yevara M. 2000. Flora y vegetación en la región central del departamento de Tarija, Bolivia. Apuntes sobre los ecosistemas originales y de reemplazo. Documento publicado dentro el marco del Proyecto Policies for Sustaining Environments and Livelihoods in Peru, Bolivia and Argentina- Ambiente y sociedad en los Andes: políticas y estrategias. Disponible vía internet en www. geog. leeds. ac. uk/ research/andes/fragenv. htm. La Paz, Bolivia.

Beck S, Paniagua-Zambrana NY, Yevara M. 2000. Flora y vegetación en la Quebrada de Humahuaca (Prov. Jujuy, Argentina) Apuntes preliminares para las áreas de Rodero, Tilcara y Volcán. Documento publicado dentro el marco del Proyecto Policies for Sustaining Environments and Livelihoods in Peru, Bolivia and Argentina- Ambiente y sociedad en los Andes: políticas y estrategias. Disponible vía internet en www. geog. leeds. ac. uk/ research/andes/fragenv. htm. La Paz, Bolivia.

Beck S, Paniagua-Zambrana NY, Yevara M. 2003. Flora y Vegetación: Las áreas de Rodero, Tilcara y Volcan. In: La Quebrada, C. Reboratti (ed.) 47-70 pp.

Beck S, Paniagua-Zambrana NY. 2010. Valle de Zongo y zonas aledañas: Paisajes naturales y pisos de vegetación. In: Ergueta Sandoval P. \& K. Aranda Alvarez (eds.). Transitando la Diversidad: Paisajes Naturales y Culturales dl municipio de La Paz. TROPICO, Municipio de La Paz, FUNDESNAP. 192 pp.

Beck S, Sarmiento J, Paniagua-Zambrana NY, Miranda C, Ribera MO. 2000. Humedales de Bolivia, una aproximación a su conocimiento actual. En memorias del Homenaje al Dr. H.C. Troels Myndel Pedersen, Universidad Nacional del Nordeste, Corrientes, Argentina. 
Bhandari J, GC, DB, Sunita D, Thapa N, Kunwar RM, Jan HA, Abbasi AM, Bussmann RW, Paniagua-Zambrana NY. 2021. Bauhinia variegata L., Bauhinia vahlii Wight \& Arn. In: Kunwar RW, Sher H, Bussmann RW. Ethnobotany of the Himalayas. Springer, Cham. doi: 10.1007/978-3-030-57408-6_34, pp. 327-336.

Bhandari KB, Kunwar B, Kunwar RM, Bussmann RW, Paniagua-Zambrana NY. 2021. Rhamnus purpurea Edgew., Rhamnus virgatus Roxb. In: Kunwar RW, Sher H, Bussmann RW. Ethnobotany of the Himalayas. Springer, Cham. doi: 10.1007/978-3-030-57408-6_202, pp. 1655-1664.

Bhandari KB, Kutal D, Kunwar RM, Jan HA, Bussmann RW, Paniagua-Zambrana NY. 2021. Persicaria amphibia (L.) S.F. Gray, Persicaria barbata (L.) H. Hara, Persicaria capitata (Buch.-Ham. ex D. Don) H. Gross., Persicaria hydropiper (L.) Spach, Persicaria nepalensis (Meisn.) H. Gross In: Kunwar RW, Sher H, Bussmann RW. Ethnobotany of the Himalayas. Springer, Cham. doi: 10.1007/978-3-030-57408-6_180, pp. 1477-1490.

Bhandari KB, Subedi S, Kunwar RM, Bussmann RW, Paniagua-Zambrana NY. 2021. Grewia disperma Rottler ex Spreng. In: Kunwar RW, Sher H, Bussmann RW. Ethnobotany of the Himalayas. Springer, Cham. doi: 10.1007/978-3030-57408-6_112, pp. 1001-1014.

Bhandari P, Kumar Shrestha, K, Kunwar RM, Bussmann RW, Paniagua-Zambrana NY. 2021. Lyonia ovalifolia (Wall.) Drude In: Kunwar RW, Sher H, Bussmann RW. Ethnobotany of the Himalayas. Springer, Cham. doi: 10.1007/978-3030-57408-6_145, pp. 1215-1222.

Bhatt MD, Kunwar RM, Abbasi AM, Bussmann RW, Paniagua-Zambrana NY. 2021. Skimmia anquetilia Tayl. \& Airy Shaw, Skimmia arborescens T. Anderson ex Gamble, Skimmia laureola (DC.) Sieb. \& Zucc. ex Walp., Skimmia melanocarpa Rehder \& E. H. Wilson, Skimmia multinervia Huang. In: Kunwar RW, Sher H, Bussmann RW. Ethnobotany of the Himalayas. Springer, Cham. doi: 10.1007/978-3-030-57408-6_228, pp. 1861-1868.

Bhatt MD, Kunwar RM, Bussmann RW, Paniagua-Zambrana NY. 2021. Acmella calva (DC.) R.K. Jansen In: Kunwar RW, Sher H, Bussmann RW. Ethnobotany of the Himalayas. Springer, Cham. doi: 10.1007/978-3-030-57408-6_9, pp. 133-138.

Bhatt MD, Kunwar RM, Bussmann RW, Paniagua-Zambrana NY. 2021. Evolvulus alsinoides (L.) L. In: Kunwar RW, Sher H, Bussmann RW. Ethnobotany of the Himalayas. Springer, Cham. doi: 10.1007/978-3-030-57408-6_99, pp. 917926.

Bhatt, MD, Kunwar RM, Basnyat B, Bussmann RW, Paniagua-Zambrana NY 2021. Alstonia scholaris (L.) R. Br. In: Kunwar RW, Sher H, Bussmann RW. Ethnobotany of the Himalayas. Springer, Cham. doi: 10.1007/978-3-030-574086_17, pp. 203-212.

Bhatta GD, Kunwar RM, Bussmann RW. 2021. Pyracantha crenulata (D. Don) M. Roem. In: Kunwar RW, Sher H, Bussmann RW. Ethnobotany of the Himalayas. Springer, Cham. doi: 10.1007/978-3-030-57408-6_199, pp. 1613-1618.

Bhatta S, Joshi LR, Khakurel D, Kunwar RM, Bussmann RW. 2021. Sapindus mukorossi Gaertn. In: Kunwar RW, Sher H, Bussmann RW. Ethnobotany of the Himalayas. Springer, Cham. doi: 10.1007/978-3-030-57408-6_214, pp. 1775-1784.

Bhatta S, Joshi LR, Kunwar RM, Bussmann RW, Paniagua-Zambrana NY. 2021. Smilax aspera L., Smilax ovalifolia Roxb. In: Kunwar RW, Sher H, Bussmann RW. Ethnobotany of the Himalayas. Springer, Cham. doi: 10.1007/978-3030-57408-6_229, pp. 1869-1880.

Bhattara, B, Chikanbanjar R, Luintel S, Gyawali S, Kunwar RM, Bussmann RW. 2021. Semecarpus anacardium L. f. In: Kunwar RW, Sher H, Bussmann RW. Ethnobotany of the Himalayas. Springer, Cham. doi: 10.1007/978-3-030-574086_224, pp. 1837-1844.

Bhattarai B, Chikanbanjar R, Kunwar RM, Bussmann RW, Paniagua-Zambrana NY. 2021. Diploknema butyracea (Roxb.) H.J. Lam. In: Kunwar RW, Sher H, Bussmann RW. Ethnobotany of the Himalayas. Springer, Cham. doi: 10.1007/978-3-030-57408-6_84, pp. 779-788.

Bhattarai KR, Kunwar RM, Bussmann RW, Paniagua-Zambrana NY. 2021. Erythrina arborescens Roxb. In: Kunwar RW, Sher H, Bussmann RW. Ethnobotany of the Himalayas. Springer, Cham. doi: 10.1007/978-3-030-57408-6_96, pp. 897-902.

Bhattarai P, Adhikari YP, Kunwar RM, Bussmann RW. 2021. Pleione humilis (Sm.) D. Don In: Kunwar RW, Sher H, Bussmann RW. Ethnobotany of the Himalayas. Springer, Cham. doi: 10.1007/978-3-030-57408-6_190, pp. 1555-1558. 
Bhattarai R, Sher H, Ur Rahman I, Kunwar RM, Ali M, Bussmann RW, Paniagua-Zambrana NY. 2021. Polygonatum cirrhifolium (Wall.) Royle, Polygonatum multiflorum (L.) All., Polygonatum verticillatum (L.) All. In: Kunwar RW, Sher H, Bussmann RW. Ethnobotany of the Himalayas. Springer, Cham. doi: 10.1007/978-3-030-57408-6_193, pp. 15691578.

Bhattarai S, Kunwar RM, Bussmann RW, Paniagua-Zambrana NY 2021. Engelhardia spicata Lesch. ex Blume In: Kunwar RW, Sher H, Bussmann RW. Ethnobotany of the Himalayas. Springer, Cham. doi: 10.1007/978-3-030-574086_91, pp. 863-866.

Bhattarai S, Kunwar RM, Bussmann RW, Paniagua-Zambrana NY. 2021. Osmanthus fragrans (Thunb.) Lour. In: Kunwar RW, Sher H, Bussmann RW. Ethnobotany of the Himalayas. Springer, Cham. doi: 10.1007/978-3-030-574086_169, pp. 1409-1414.

Bindu KC, Gyawal S, Luintel, S, Sharma HP, Kunwar RM, Bussmann RW, Paniagua-Zambrana NY. 2021. Sorbus cuspidata (Spach) Hedl. In: Kunwar RW, Sher H, Bussmann RW. Ethnobotany of the Himalayas. Springer, Cham. doi: 10.1007/978-3-030-57408-6_233, pp. 1917-1926

Bridgewater P, Upadhaya S, Poudyal B, Kunwar RM, Bussmann RW, Paniagua-Zambrana NY 2021. Nigella sativa L. In: Kunwar RW, Sher H, Bussmann RW. Ethnobotany of the Himalayas. Springer, Cham. doi: 10.1007/978-3-03057408-6_162, pp. 1359-1368.

Budha-Magar, S, Sher H, Kunwar RM, Bussmann RW, Ur Rahman, I. 2021. Saussurea albescens (DC.) Sch., Saussurea lappa (Decne.) Sch. Bip, Saussurea obvallata (DC.) Sch. Bip. In: Kunwar RW, Sher H, Bussmann RW. Ethnobotany of the Himalayas. Springer, Cham. doi: 10.1007/978-3-030-57408-6_217, pp. 1795-1804.

Bussmann RW, Batsatsashvili K, Kikvidze Z, 2020. Amaranthus hybridus L., Amaranthus palmeri S. Watson, Amaranthus spinosus L., Amaranthus retrofexus L., Atriplex hortensis L. In: Batsatsashvili K, Kikvidze Z, Bussmann RW (Eds.) Ethnobotany of Mountain Regions Central Asia and Altai. Springer International Publishing, Cham. doi: 10.1007/978-3-319-77087-1_18-1

Bussmann RW, Batsatsashvili K, Kikvidze Z, Khutsishvili M, Maisaia I, Sikharulidze S, Tchelidze D, Paniagua-Zambrana NY. 2017. Ethnobotany of the Caucasus - the region. In: Bussmann RW (ed.) Ethnobotany of the Caucasus, Springer International Publishing, Cham. doi: 10.1007/978-3-319-49412-8_17

Bussmann RW, Batsatsashvili K, Kikvidze Z, Khutsishvili M, Maisaia I, Sikharulidze S, Tchelidze D, Paniagua-Zambrana NY. 2017. Ethnobotany of the Caucasus - Georgia. In: Bussmann RW (ed.) Ethnobotany of the Caucasus, Springer International Publishing, Cham. doi: 10.1007/978-3-319-49412-8_19

Bussmann RW, Batsatsashvili K, Kikvidze Z, Paniagua-Zambrana NY, Khutsishvili M, Maisaia I, Sikharulidze S, Tchelidze D. 2020. Amaranthus hybridus L., Amaranthus palmeri S. Watson, Amaranthus spinosus L., Amaranthus retrofexus L., Atriplex hortensis L. In: Batsatsashvili K, Kikvidze Z, Bussmann RW (Eds.) Ethnobotany of Mountain Regions Far Eastern Europe. Springer International Publishing, Cham. doi: 10.1007/978-3-319-77087-1_20-2

Bussmann RW, Batsatsashvili K, Kikvidze Z, Paniagua-Zambrana NY, Khutsishvili M, Maisaia I, Sikharulidze S, Tchelidze D. 2020. Angelica adzharica Pimenov, Angelica tatianae Bordz. In: Batsatsashvili K, Kikvidze Z, Bussmann RW (Eds.) Ethnobotany of Mountain Regions Far Eastern Europe. Springer International Publishing, Cham. doi: 10.1007/978-3-319-77088-8_11-2

Bussmann RW, Batsatsashvili K, Kikvidze Z, Paniagua-Zambrana NY, Moazzami Farida SH, Ghorbani A, Khutsishvili M, Maisaia I, Sikharulidze S, Tchelidze D. 2020. Apium graveolens L. In: Batsatsashvili K, Kikvidze Z, Bussmann RW (Eds.) Ethnobotany of Mountain Regions Far Eastern Europe. Springer International Publishing, Cham. doi: 10.1007/978-3-319-77088-8_13-2

Bussmann RW, Batsatsashvili K, Kikvidze Z, Paniagua-Zambrana NY, Khutsishvili M, Maisaia I, Sikharulidze S, Tchelidze D. 2020. Arctium lappa L. In: Batsatsashvili K, Kikvidze Z, Bussmann RW (Eds.) Ethnobotany of Mountain Regions Far Eastern Europe. Springer International Publishing, Cham. doi: 10.1007/978-3-319-77088-8_14-2

Bussmann RW, Batsatsashvili K, Kikvidze Z, Paniagua-Zambrana NY, Khutsishvili M, Maisaia I, Sikharulidze S, Tchelidze D. 2020. Beta vulgaris L. In: Batsatsashvili K, Kikvidze Z, Bussmann RW (Eds.) Ethnobotany of Mountain Regions Far Eastern Europe. Springer International Publishing, Cham. doi: 10.1007/978-3-319-77088-8_22-2

Bussmann RW, Batsatsashvili K, Kikvidze Z, Paniagua-Zambrana NY, Khutsishvili M, Maisaia I, Sikharulidze S, Tchelidze D. 2020. Bilacunaria caspia (DC.) Pimenov \& V.N. Tikhom. In: Batsatsashvili K, Kikvidze Z, Bussmann RW 
(Eds.) Ethnobotany of Mountain Regions Far Eastern Europe. Springer International Publishing, Cham. doi: 10.1007/978-3-319-77088-8_23-2

Bussmann RW, Batsatsashvili K, Kikvidze Z, Paniagua-Zambrana NY, Khutsishvili M, Maisaia I, Sikharulidze S, Tchelidze D. 2020. Bistorta officinalis Delarbre, Polygonum carneum C. Koch. In: Batsatsashvili K, Kikvidze Z, Bussmann RW (Eds.) Ethnobotany of Mountain Regions Far Eastern Europe. Springer International Publishing, Cham. doi: 10.1007/978-3-319-77088-8_24-2

Bussmann RW, Batsatsashvili K, Kikvidze Z, Paniagua-Zambrana NY, Khutsishvili M, Maisaia I, Sikharulidze S, Tchelidze D. 2020. Campanula biebersteiniana C.A. Mey., Campanula rapunculoides L. In: Batsatsashvili K, Kikvidze Z, Bussmann RW (Eds.) Ethnobotany of Mountain Regions Far Eastern Europe. Springer International Publishing, Cham. doi: 10.1007/978-3-319-77088-8_32-2

Bussmann RW, Batsatsashvili K, Kikvidze Z, Paniagua-Zambrana NY, Khutsishvili M, Maisaia I, Sikharulidze S, Tchelidze D. 2020. Helianthus annuus L., Helianthus tuberosus L. In: Batsatsashvili K, Kikvidze Z, Bussmann RW (Eds.) Ethnobotany of Mountain Regions Far Eastern Europe. Springer International Publishing, Cham. doi: 10.1007/978-3319-77088-8_67-2

Bussmann RW, Batsatsashvili K, Kikvidze Z, Paniagua-Zambrana NY, Moazzami Farida SH, Ghorbani A, Khutsishvili M, Maisaia I, Sikharulidze S, Tchelidze D. 2020. Heracleum asperum M. B. Fl., Heracleum leskovii A. Grossh., Heracleum mantegazzianum Sommier \& Levier, Heracleum persicum L., Heracleum sibiricum L., Heracleum sosnowskyi Manden., Heracleum spondylium L., Heracleum wilhelmsii Fich. \& Ave-Lall. In: Batsatsashvili K, Kikvidze Z, Bussmann RW (Eds.) Ethnobotany of Mountain Regions Far Eastern Europe. Springer International Publishing, Cham.

Bussmann RW, Batsatsashvili K, Kikvidze Z, Paniagua-Zambrana NY, Khutsishvili M, Maisaia I, Sikharulidze S, Tchelidze D. 2020. Hypericum perforatum L. In: Batsatsashvili K, Kikvidze Z, Bussmann RW (Eds.) Ethnobotany of Mountain Regions Far Eastern Europe. Springer International Publishing, Cham. doi: 10.1007/978-3-319-770888_75-2

Bussmann RW, Batsatsashvili K, Kikvidze Z, Paniagua-Zambrana NY, Khutsishvili M, Maisaia I, Sikharulidze S, Tchelidze D. 2020. Malus orientalis Uglitzk., Malus pumila Mill. In: Batsatsashvili K, Kikvidze Z, Bussmann RW (Eds.) Ethnobotany of Mountain Regions Far Eastern Europe. Springer International Publishing, Cham. doi: 10.1007/978-3319-77088-8_86-2

Bussmann RW, Batsatsashvili K, Kikvidze Z, Paniagua-Zambrana NY, Khutsishvili M, Maisaia I, Sikharulidze S, Tchelidze D. 2020. Morus alba L. In: Batsatsashvili K, Kikvidze Z, Bussmann RW (Eds.) Ethnobotany of Mountain Regions Far Eastern Europe. Springer International Publishing, Cham. doi: 10.1007/978-3-319-77088-8_91-2

Bussmann RW, Batsatsashvili K, Kikvidze Z, Paniagua-Zambrana NY, Khutsishvili M, Maisaia I, Sikharulidze S, Tchelidze D. 2020. Origanum vulgare L. In: Batsatsashvili K, Kikvidze Z, Bussmann RW (Eds.) Ethnobotany of Mountain Regions Far Eastern Europe. Springer International Publishing, Cham. doi: 10.1007/978-3-319-77088-8_97-2

Bussmann RW, Batsatsashvili K, Kikvidze Z, Paniagua-Zambrana NY, Khutsishvili M, Maisaia I, Sikharulidze S, Tchelidze D. 2020. Panicum milanjanum Rendle. In: Batsatsashvili K, Kikvidze Z, Bussmann RW (Eds.) Ethnobotany of Mountain Regions Far Eastern Europe. Springer International Publishing, Cham. doi: 10.1007/978-3-319-770888_98-2

Bussmann RW, Batsatsashvili K, Kikvidze Z, Paniagua-Zambrana NY, Khutsishvili M, Maisaia I, Sikharulidze S, Tchelidze D. 2020. Petasites albus (L.) Gaertn., Petasites fominii Bordz., Petasites vulgaris Desf. In: Batsatsashvili K, Kikvidze Z, Bussmann RW (Eds.) Ethnobotany of Mountain Regions Far Eastern Europe. Springer International Publishing, Cham. doi: 10.1007/978-3-319-77088-8_101-2

Bussmann RW, Batsatsashvili K, Kikvidze Z, Paniagua-Zambrana NY, Khutsishvili M, Maisaia I, Sikharulidze S, Tchelidze D. 2020. Phaseolus sativus Royle, Phaseolus vulgaris L. In: Batsatsashvili K, Kikvidze Z, Bussmann RW (Eds.) Ethnobotany of Mountain Regions Far Eastern Europe. Springer International Publishing, Cham. doi: 10.1007/978-3319-77088-8_103-2

Bussmann RW, Batsatsashvili K, Kikvidze Z, Paniagua-Zambrana NY, Ghorbani A, Nasab Farzaneh K, Khutsishvili M, Maisaia I, Sikharulidze S, Tchelidze D. 2020. Polygonum aviculare L. In: Batsatsashvili K, Kikvidze Z, Bussmann RW (Eds.) Ethnobotany of Mountain Regions Far Eastern Europe. Springer International Publishing, Cham. doi: 10.1007/978-3-319-77088-8_108-2 
Bussmann RW, Batsatsashvili K, Kikvidze Z, Paniagua-Zambrana NY, Khutsishvili M, Maisaia I, Sikharulidze S, Tchelidze D. 2020. Prunus avium (L.) L., Prunus cerasus L., Prunus divaricata Ledeb., Prunus domestica L., Prunus insititia L., Prunus laurocerasus L., Prunus padus L., Prunus vachuschtii Bregadze. In: Batsatsashvili K, Kikvidze Z, Bussmann RW (Eds.) Ethnobotany of Mountain Regions Far Eastern Europe. Springer International Publishing, Cham. doi: 10.1007/978-3-319-77088-8_110-2

Bussmann RW, Batsatsashvili K, Kikvidze Z, Paniagua-Zambrana NY, Khutsishvili M, Maisaia I, Sikharulidze S, Tchelidze D. 2020. Rhododendron caucasicum Pall., Rhododendrum luteum Sweet, Rhododendrum ponticum L. In: Batsatsashvili K, Kikvidze Z, Bussmann RW (Eds.) Ethnobotany of Mountain Regions Far Eastern Europe. Springer International Publishing, Cham. doi: 10.1007/978-3-319-77088-8_115-2

Bussmann RW, Batsatsashvili K, Kikvidze Z, Paniagua-Zambrana NY, Khutsishvili M, Maisaia I, Sikharulidze S, Tchelidze D. 2020. Ribes biebersteinii Berland. ex DC., Ribes nigrum L., Ribes odoratum H.L. Wendl., Ribes orientalis Desf., Ribes rubrum L., Ribes uva-crispa L. In: Batsatsashvili K, Kikvidze Z, Bussmann RW (Eds.) Ethnobotany of Mountain Regions Far Eastern Europe. Springer International Publishing, Cham. doi: 10.1007/978-3-319-770888_116-2

Bussmann RW, Batsatsashvili K, Kikvidze Z, Paniagua-Zambrana NY, Ghorbani A, Nasab Farzaneh K, Khutsishvili M, Maisaia I, Sikharulidze S, Tchelidze D. 2020. Rosa canina L., Rosa pimpinellifolia Boiss. In: Batsatsashvili K, Kikvidze Z, Bussmann RW (Eds.) Ethnobotany of Mountain Regions Far Eastern Europe. Springer International Publishing, Cham. doi: 10.1007/978-3-319-77088-8_118-2

Bussmann RW, Batsatsashvili K, Kikvidze Z, Paniagua-Zambrana NY, Khutsishvili M, Maisaia I, Sikharulidze S, Tchelidze D. 2020. Seseli transcaucasicum Pimenov \& Sdobina. In: Batsatsashvili K, Kikvidze Z, Bussmann RW (Eds.) Ethnobotany of Mountain Regions Far Eastern Europe. Springer International Publishing, Cham. doi: 10.1007/978-3319-77088-8_126-2

Bussmann RW, Batsatsashvili K, Kikvidze Z, Paniagua-Zambrana NY, Khutsishvili M, Maisaia I, Sikharulidze S, Tchelidze D. 2020. Smilax excelsa L. In: Batsatsashvili K, Kikvidze Z, Bussmann RW (Eds.) Ethnobotany of Mountain Regions Far Eastern Europe. Springer International Publishing, Cham. doi: 10.1007/978-3-319-77088-8_150-2

Bussmann RW, Batsatsashvili K, Kikvidze Z, Paniagua-Zambrana NY, Khutsishvili M, Maisaia I, Sikharulidze S, Tchelidze D. 2020. Solanum melongena L., Solanum nigrum L., Solanum tuberosum L. In: Batsatsashvili K, Kikvidze Z, Bussmann RW (Eds.) Ethnobotany of Mountain Regions Far Eastern Europe. Springer International Publishing, Cham. doi: 10.1007/978-3-319-77088-8_128-2

Bussmann RW, Batsatsashvili K, Kikvidze Z, Paniagua-Zambrana NY, Khutsishvili M, Maisaia I, Sikharulidze S, Tchelidze D. 2020. Ulmus glabra Huds. In: Batsatsashvili K, Kikvidze Z, Bussmann RW (Eds.) Ethnobotany of Mountain Regions Far Eastern Europe. Springer International Publishing, Cham. doi: 10.1007/978-3-319-77088-8_137-2

Bussmann RW, Batsatsashvili K, Kikvidze Z, Paniagua-Zambrana NY, Khutsishvili M, Maisaia I, Sikharulidze S, Tchelidze D. 2020. Vaccinium arctostaphylos L., Vaccinium myrtillus L., Vaccinium oxycoccos L., Vaccinium vitis-idaea L. In: Batsatsashvili K, Kikvidze Z, Bussmann RW (Eds.) Ethnobotany of Mountain Regions Far Eastern Europe. Springer International Publishing, Cham.

Bussmann RW, Batsatsashvili K, Kikvidze Z, Paniagua-Zambrana NY, Khutsishvili M, Maisaia I, Sikharulidze S, Tchelidze D. 2020. Verbascum sp., Verbascum thapsus L. In: Batsatsashvili K, Kikvidze Z, Bussmann RW (Eds.) Ethnobotany of Mountain Regions Far Eastern Europe. Springer International Publishing, Cham. doi: 10.1007/978-3319-77088-8_141-2

Bussmann RW, Batsatsashvili K, Kikvidze Z, Paniagua-Zambrana NY, Khutsishvili M, Maisaia I, Sikharulidze S, Tchelidze D. 2020. Viburnum lantana L., Viburnum opulus L. In: Batsatsashvili K, Kikvidze Z, Bussmann RW (Eds.) Ethnobotany of Mountain Regions Far Eastern Europe. Springer International Publishing, Cham. doi: 10.1007/978-3319-77088-8_142-2

Bussmann RW, Batsatsashvili K, Kikvidze Z, Paniagua-Zambrana NY, Khutsishvili M, Maisaia I, Sikharulidze S, Tchelidze D. 2020. Vicia faba L., Vicia sativa L. n: Batsatsashvili K, Kikvidze Z, Bussmann RW (Eds.) Ethnobotany of Mountain Regions Far Eastern Europe. Springer International Publishing, Cham. doi: 10.1007/978-3-319-770888_143-2

Bussmann RW, Batsatsashvili K, Kikvidze Z, Paniagua-Zambrana NY, Khutsishvili M, Maisaia I, Sikharulidze S, Tchelidze D. 2020. Chaerophyllum aureum L., Chaerophyllum bulbosum L., Chaerophyllum hirsutum L. In: 
Batsatsashvili K, Kikvidze Z, Bussmann RW (Eds.) Ethnobotany of Mountain Regions Far Eastern Europe. Springer International Publishing, Cham.

Bussmann RW, Batsatsashvili K, Kikvidze Z, Paniagua-Zambrana NY, Khutsishvili M, Maisaia I, Sikharulidze S, Tchelidze D. 2020. Aruncus dioicus (Walter) Fernald. In: Batsatsashvili K, Kikvidze Z, Bussmann RW (Eds.) Ethnobotany of Mountain Regions Far Eastern Europe. Springer International Publishing, Cham. doi: 10.1007/978-3-319-770888_18-2

Bussmann RW, Batsatsashvili K, Kikvidze Z, Paniagua-Zambrana NY, Khutsishvili M, Maisaia I, Sikharulidze S, Tchelidze D. 2020. Rubus fruticosus L., Rubus idaeus L., Rubus saxatilis L. In: Batsatsashvili K, Kikvidze Z, Bussmann RW (Eds.) Ethnobotany of Mountain Regions Far Eastern Europe. Springer International Publishing, Cham. doi: 10.1007/978-3-319-77088-8_119-2

Bussmann RW, Batsatsashvili K, Kikvidze Z, Paniagua-Zambrana NY, Ghorbani A, Nasab Farzaneh K, Khutsishvili M, Maisaia I, Sikharulidze S, Tchelidze D. 2020. Glycyrrhiza glabra L. In: Batsatsashvili K, Kikvidze Z, Bussmann RW (Eds.) Ethnobotany of Mountain Regions Far Eastern Europe. Springer International Publishing, Cham. doi: 10.1007/978-3319-77088-8_65-1

Bussmann RW, Batsatsashvili K, Kikvidze Z, Paniagua-Zambrana NY, Khutsishvili M, Maisaia I, Sikharulidze S, Tchelidze D. 2020. Gentiana cruciata L., Gentiana septemfida Pall. In: Batsatsashvili K, Kikvidze Z, Bussmann RW (Eds.) Ethnobotany of Mountain Regions Far Eastern Europe. Springer International Publishing, Cham. doi: 10.1007/978-3319-77088-8_77-2

Bussmann RW, Batsatsashvili K, Kikvidze Z, Paniagua-Zambrana NY, Khutsishvili M, Maisaia I, Sikharulidze S, Tchelidze D. 2020. Helichrysum arenarium (L.) Moench, Helichrysum rubicumdum (K. Koch) Bornm. In: Batsatsashvili K, Kikvidze Z, Bussmann RW (Eds.) Ethnobotany of Mountain Regions Far Eastern Europe. Springer International Publishing, Cham. doi: 10.1007/978-3-319-77088-8_68-2

Bussmann RW, Batsatsashvili K, Kikvidze Z, Paniagua-Zambrana NY, Khutsishvili M, Maisaia I, Sikharulidze S, Tchelidze D. 2020. Juniperus communis L., Juniperus depressa Raf., Juniperus hemispherica J. Presl \& C. Presl, Juniperus oblonga M. Bieb., Juniperus sabina L. In: Batsatsashvili K, Kikvidze Z, Bussmann RW (Eds.) Ethnobotany of Mountain Regions Far Eastern Europe. Springer International Publishing, Cham. doi: 10.1007/978-3-319-770888_77-2

Bussmann RW, Batsatsashvili K, Kikvidze Z, Paniagua-Zambrana NY, Khutsishvili M, Maisaia I, Sikharulidze S, Tchelidze D. 2020. Aethusa cynapium L. In: Batsatsashvili K, Kikvidze Z, Bussmann RW (Eds.) Ethnobotany of Mountain Regions Far Eastern Europe. Springer International Publishing, Cham. doi: 10.1007/978-3-319-77088-8_6-2

Bussmann RW, Batsatsashvili K, Kikvidze Z, Paniagua-Zambrana NY, Khutsishvili M, Maisaia I, Sikharulidze S, Tchelidze D. 2020. Lycopersicon esculentum Mill. In: Batsatsashvili K, Kikvidze Z, Bussmann RW (Eds.) Ethnobotany of Mountain Regions Far Eastern Europe. Springer International Publishing, Cham. doi: 10.1007/978-3-319-770888_85-2

Bussmann RW, Batsatsashvili K, Kikvidze Z, Paniagua-Zambrana NY, Ghorbani A, Nasab Farzaneh K, Khutsishvili M, Maisaia I, Sikharulidze S, Tchelidze D. 2020. Achillea grandiflora M. Bieb., Achillea micrantha Willd., Achillea millefolium L., Achillea nobilis L., Achillea ptarmicifolia (Willd.) Rupr. ex Heimerl In: Batsatsashvili K, Kikvidze Z, Bussmann RW (Eds.) Ethnobotany of Mountain Regions Far Eastern Europe. Springer International Publishing, Cham. doi: 10.1007/978-3-319-77088-8_5-2

Bussmann RW, Batsatsashvili K, Kikvidze Z, Paniagua-Zambrana NY, Ghorbani A, Nasab Farzaneh K, Khutsishvili M, Maisaia I, Sikharulidze S, Tchelidze D. 2020. Artemisia absinthium L., Artemisia annua L., Artemisia dracunculus L., Artemisia leucodes Schrenk, Artemisia scoparia Waldst. \& Kit., Artemisia vulgaris L., Eclipta prostrata (L.) L. In: Batsatsashvili K, Kikvidze Z, Bussmann RW (Eds.) Ethnobotany of Mountain Regions Far Eastern Europe. Springer International Publishing, Cham. doi: 10.1007/978-3-319-77088-8_16-2

Bussmann RW, Batsatsashvili K, Kikvidze Z, Paniagua-Zambrana NY, Khutsishvili M, Maisaia I, Sikharulidze S, Tchelidze D. 2020. Brassica rapa L. In: Batsatsashvili K, Kikvidze Z, Bussmann RW (Eds.) Ethnobotany of Mountain Regions Far Eastern Europe. Springer International Publishing, Cham. doi: 10.1007/978-3-319-77088-8_28-2

Bussmann RW, Batsatsashvili K, Kikvidze Z, Paniagua-Zambrana NY, Khutsishvili M, Maisaia I, Sikharulidze S, Tchelidze D. 2020. Calendula officinalis L. In: Batsatsashvili K, Kikvidze Z, Bussmann RW (Eds.) Ethnobotany of 
Mountain Regions Far Eastern Europe. Springer International Publishing, Cham. doi: 10.1007/978-3-319-770888_31-2

Bussmann RW, Batsatsashvili K, Kikvidze Z, Paniagua-Zambrana NY, Ghorbani A, Nasab Farzaneh K, Khutsishvili M, Maisaia I, Sikharulidze S, Tchelidze D. 2020. Capsella bursa-pastoris (L.) Medik. In: Batsatsashvili K, Kikvidze Z, Bussmann RW (Eds.) Ethnobotany of Mountain Regions Far Eastern Europe. Springer International Publishing, Cham. doi: 10.1007/978-3-319-77088-8_34-2

Bussmann RW, Batsatsashvili K, Kikvidze Z, Paniagua-Zambrana NY, Khutsishvili M, Maisaia I, Sikharulidze S, Tchelidze D. 2020. Cardamine hirsuta L. In: Batsatsashvili K, Kikvidze Z, Bussmann RW (Eds.) Ethnobotany of Mountain Regions Far Eastern Europe. Springer International Publishing, Cham. doi: 10.1007/978-3-319-77088-8_36-2

Bussmann RW, Batsatsashvili K, Kikvidze Z, Paniagua-Zambrana NY, Ghorbani A, Khutsishvili M, Maisaia I, Sikharulidze S, Tchelidze D. 2020. Cichorium intybus L. In: Batsatsashvili K, Kikvidze Z, Bussmann RW (Eds.) Ethnobotany of Mountain Regions Far Eastern Europe. Springer International Publishing, Cham. doi: 10.1007/978-3319-77088-8_40-2

Bussmann RW, Batsatsashvili K, Kikvidze Z, Paniagua-Zambrana NY, Moazzami Farida SH, Ghorbani A, Khutsishvili M, Maisaia I, Sikharulidze S, Tchelidze D. 2020. Coriandrum sativum L. In: Batsatsashvili K, Kikvidze Z, Bussmann RW (Eds.) Ethnobotany of Mountain Regions Far Eastern Europe. Springer International Publishing, Cham. doi: 10.1007/978-3-319-77088-8_43-2

Bussmann RW, Batsatsashvili K, Kikvidze Z, Paniagua-Zambrana NY, Khutsishvili M, Maisaia I, Sikharulidze S, Tchelidze D. 2020. Cornus australis C.A. Mey., Cornus mas L. In: Batsatsashvili K, Kikvidze Z, Bussmann RW (Eds.) Ethnobotany of Mountain Regions Far Eastern Europe. Springer International Publishing, Cham. doi: 10.1007/978-3319-77088-8_44-2

Bussmann RW, Batsatsashvili K, Kikvidze Z, Paniagua-Zambrana NY, Khutsishvili M, Maisaia I, Sikharulidze S, Tchelidze D. 2020. Equisetum arvense L., Equisetum telmateja Ehrh. In: Batsatsashvili K, Kikvidze Z, Bussmann RW (Eds.) Ethnobotany of Mountain Regions Far Eastern Europe. Springer International Publishing, Cham. doi: 10.1007/978-3-319-77088-8_55-3

Bussmann RW, Batsatsashvili K, Kikvidze Z, Paniagua-Zambrana NY, Khutsishvili M, Maisaia I, Sikharulidze S, Tchelidze D. 2020. Eruca sativa Mill. In: Batsatsashvili K, Kikvidze Z, Bussmann RW (Eds.) Ethnobotany of Mountain Regions Far Eastern Europe. Springer International Publishing, Cham. doi: 10.1007/978-3-319-77088-8_56-2

Bussmann RW, Batsatsashvili K, Kikvidze Z, Paniagua-Zambrana NY, Khutsishvili M, Maisaia I, Sikharulidze S, Tchelidze D. 2020. Erysimum cheiri (L.) Crantz. In: Batsatsashvili K, Kikvidze Z, Bussmann RW (Eds.) Ethnobotany of Mountain Regions Far Eastern Europe. Springer International Publishing, Cham. doi: 10.1007/978-3-319-770888_57-1

Bussmann RW, Batsatsashvili K, Kikvidze Z, Paniagua-Zambrana NY, Moazzami Farida SH, Ghorbani A, Khutsishvili M, Maisaia I, Sikharulidze S, Tchelidze D. 2020. Ferula assa-foetida L. In: Batsatsashvili K, Kikvidze Z, Bussmann RW (Eds.) Ethnobotany of Mountain Regions Far Eastern Europe. Springer International Publishing, Cham. doi: 10.1007/978-3-319-77088-8_59-1

Bussmann RW, Batsatsashvili K, Kikvidze Z, Paniagua-Zambrana NY, Khutsishvili M, Maisaia I, Sikharulidze S, Tchelidze D. 2020. Galega orientalis Lam. In: Batsatsashvili K, Kikvidze Z, Bussmann RW (Eds.) Ethnobotany of Mountain Regions Far Eastern Europe. Springer International Publishing, Cham. doi: 10.1007/978-3-319-770888_63-2

Bussmann RW, Batsatsashvili K, Kikvidze Z, Paniagua-Zambrana NY, Khutsishvili M, Maisaia I, Sikharulidze S, Tchelidze D. 2020. Humulus lupulus L. In: Batsatsashvili K, Kikvidze Z, Bussmann RW (Eds.) Ethnobotany of Mountain Regions Far Eastern Europe. Springer International Publishing, Cham. doi: 10.1007/978-3-319-77088-8_73-2

Bussmann RW, Batsatsashvili K, Kikvidze Z, Paniagua-Zambrana NY, Khutsishvili M, Maisaia I, Sikharulidze S, Tchelidze D. 2020. Lactuca sativa L., Lactuca serriola L. In: Batsatsashvili K, Kikvidze Z, Bussmann RW (Eds.) Ethnobotany of Mountain Regions Far Eastern Europe. Springer International Publishing, Cham. doi: 10.1007/978-3319-77088-8_78-2

Bussmann RW, Batsatsashvili K, Kikvidze Z, Paniagua-Zambrana NY, Khutsishvili M, Maisaia I, Sikharulidze S, Tchelidze D. 2020. Leonurus quinquelobatus Gilib. In: Batsatsashvili K, Kikvidze Z, Bussmann RW (Eds.) Ethnobotany 
of Mountain Regions Far Eastern Europe. Springer International Publishing, Cham. doi: 10.1007/978-3-319-770888_80-2

Bussmann RW, Batsatsashvili K, Kikvidze Z, Paniagua-Zambrana NY, Khutsishvili M, Maisaia I, Sikharulidze S, Tchelidze D. 2020. Ligusticum alatum Spreng. In: Batsatsashvili K, Kikvidze Z, Bussmann RW (Eds.) Ethnobotany of Mountain Regions Far Eastern Europe. Springer International Publishing, Cham. doi: 10.1007/978-3-319-770888_83-1

Bussmann RW, Batsatsashvili K, Kikvidze Z, Paniagua-Zambrana NY, Khutsishvili M, Maisaia I, Sikharulidze S, Tchelidze D. 2020. Melandrium divaricatum Fenzl. In: Batsatsashvili K, Kikvidze Z, Bussmann RW (Eds.) Ethnobotany of Mountain Regions Far Eastern Europe. Springer International Publishing, Cham. doi: 10.1007/978-3-319-770888_88-2

Bussmann RW, Batsatsashvili K, Kikvidze Z, Paniagua-Zambrana NY, Khutsishvili M, Maisaia I, Sikharulidze S, Tchelidze D. 2020. Nepeta mussinii Spreng. ex Henckel. In: Batsatsashvili K, Kikvidze Z, Bussmann RW (Eds.) Ethnobotany of Mountain Regions Far Eastern Europe. Springer International Publishing, Cham. doi: 10.1007/978-3319-77088-8_92-2

Bussmann RW, Batsatsashvili K, Kikvidze Z, Paniagua-Zambrana NY, Ghorbani A, Nasab Farzaneh K, Khutsishvili M, Maisaia I, Sikharulidze S, Tchelidze D. 2020. Peganum harmala L. In: Batsatsashvili K, Kikvidze Z, Bussmann RW (Eds.) Ethnobotany of Mountain Regions Far Eastern Europe. Springer International Publishing, Cham. doi: 10.1007/978-3319-77088-8_99-2

Bussmann RW, Batsatsashvili K, Kikvidze Z, Paniagua-Zambrana NY, Ghorbani A, Nasab Farzaneh K, Khutsishvili M, Maisaia I, Sikharulidze S, Tchelidze D. 2020. Plantago lanceolata L., Plantago major L. In: Batsatsashvili K, Kikvidze Z, Bussmann RW (Eds.) Ethnobotany of Mountain Regions Far Eastern Europe. Springer International Publishing, Cham. doi: 10.1007/978-3-319-77088-8_107-2

Bussmann RW, Batsatsashvili K, Kikvidze Z, Paniagua-Zambrana NY, Khutsishvili M, Maisaia I, Sikharulidze S, Tchelidze D. 2020. Prunus armeniaca L. In: Batsatsashvili K, Kikvidze Z, Bussmann RW (Eds.) Ethnobotany of Mountain Regions Far Eastern Europe. Springer International Publishing, Cham. doi: 10.1007/978-3-319-77088-8_149-2

Bussmann RW, Batsatsashvili K, Kikvidze Z, Paniagua-Zambrana NY, Khutsishvili M, Maisaia I, Sikharulidze S, Tchelidze D. 2020. Punica granatum L. In: Batsatsashvili K, Kikvidze Z, Bussmann RW (Eds.) Ethnobotany of Mountain Regions Far Eastern Europe. Springer International Publishing, Cham. doi: 10.1007/978-3-319-77088-8_112-2

Bussmann RW, Batsatsashvili K, Kikvidze Z, Paniagua-Zambrana NY, Khutsishvili M, Maisaia I, Sikharulidze S, Tchelidze D. 2020. Pyrus caucasica Fed., Pyrus communis L. In: Batsatsashvili K, Kikvidze Z, Bussmann RW (Eds.) Ethnobotany of Mountain Regions Far Eastern Europe. Springer International Publishing, Cham. doi: 10.1007/978-3319-77088-8_113-2

Bussmann RW, Batsatsashvili K, Kikvidze Z, Paniagua-Zambrana NY, Khutsishvili M, Maisaia I, Sikharulidze S, Tchelidze D. 2020. Raphanus sativus L. In: Batsatsashvili K, Kikvidze Z, Bussmann RW (Eds.) Ethnobotany of Mountain Regions Far Eastern Europe. Springer International Publishing, Cham. doi: 10.1007/978-3-319-77088-8_114-2

Bussmann RW, Batsatsashvili K, Kikvidze Z, Paniagua-Zambrana NY, Khutsishvili M, Maisaia I, Sikharulidze S, Tchelidze D. 2020. Robinia pseudoacacia L. In: Batsatsashvili K, Kikvidze Z, Bussmann RW (Eds.) Ethnobotany of Mountain Regions Far Eastern Europe. Springer International Publishing, Cham. doi: 10.1007/978-3-319-770888_117-2

Bussmann RW, Batsatsashvili K, Kikvidze Z, Paniagua-Zambrana NY, Khutsishvili M, Maisaia I, Sikharulidze S, Tchelidze D. 2020. Danae racemosa (L.) Moench, Ruscus hyrcanus Woron., Ruscus hypophyllum L. In: Batsatsashvili K, Kikvidze Z, Bussmann RW (Eds.) Ethnobotany of Mountain Regions Far Eastern Europe. Springer International Publishing, Cham. dopi: 10.1007/978-3-319-77088-8_120-2

Bussmann RW, Batsatsashvili K, Kikvidze Z, Paniagua-Zambrana NY, Khutsishvili M, Maisaia I, Sikharulidze S, Tchelidze D. 2020. Salix alba L., Salix caprea L. In: Batsatsashvili K, Kikvidze Z, Bussmann RW (Eds.) Ethnobotany of Mountain Regions Far Eastern Europe. Springer International Publishing, Cham. doi: 10.1007/978-3-319-770888_121-2

Bussmann RW, Batsatsashvili K, Kikvidze Z, Paniagua-Zambrana NY, Khutsishvili M, Maisaia I, Sikharulidze S, Tchelidze D. 2020. Salvia nemorosa L., Salvia verticillata L. In: Batsatsashvili K, Kikvidze Z, Bussmann RW (Eds.) 
Ethnobotany of Mountain Regions Far Eastern Europe. Springer International Publishing, Cham. doi: 10.1007/978-3319-77088-8_122-2

Bussmann RW, Batsatsashvili K, Kikvidze Z, Paniagua-Zambrana NY, Khutsishvili M, Maisaia I, Sikharulidze S, Tchelidze D. 2020. Satureja hortensis L., Satureja spicigera (C. Koch.) Boiss. In: Batsatsashvili K, Kikvidze Z, Bussmann RW (Eds.) Ethnobotany of Mountain Regions Far Eastern Europe. Springer International Publishing, Cham. doi: 10.1007/978-3-319-77088-8_123-2

Bussmann RW, Batsatsashvili K, Kikvidze Z, Paniagua-Zambrana NY, Khutsishvili M, Maisaia I, Sikharulidze S, Tchelidze D. 2020. Secale cereale L. In: Batsatsashvili K, Kikvidze Z, Bussmann RW (Eds.) Ethnobotany of Mountain Regions Far Eastern Europe. Springer International Publishing, Cham. doi: 10.1007/978-3-319-77088-8_124-2

Bussmann RW, Batsatsashvili K, Kikvidze Z, Paniagua-Zambrana NY, Khutsishvili M, Maisaia I, Sikharulidze S, Tchelidze D. 2020. Serratula quinquefolia Bieb. ex Willd. In: Batsatsashvili K, Kikvidze Z, Bussmann RW (Eds.) Ethnobotany of Mountain Regions Far Eastern Europe. Springer International Publishing, Cham. doi: 10.1007/978-3319-77088-8_125-1

Bussmann RW, Batsatsashvili K, Kikvidze Z, Paniagua-Zambrana NY, Khutsishvili M, Maisaia I, Sikharulidze S, Tchelidze D. 2020. Sinapis arvensis L. In: Batsatsashvili K, Kikvidze Z, Bussmann RW (Eds.) Ethnobotany of Mountain Regions Far Eastern Europe. Springer International Publishing, Cham. doi: 10.1007/978-3-319-77088-8_127-2

Bussmann RW, Batsatsashvili K, Kikvidze Z, Paniagua-Zambrana NY, Khutsishvili M, Maisaia I, Sikharulidze S, Tchelidze D. 2020. Sonchus asper (L.) Hill, Sonchus oleraceus L. In: Batsatsashvili K, Kikvidze Z, Bussmann RW (Eds.) Ethnobotany of Mountain Regions Far Eastern Europe. Springer International Publishing, Cham. doi: 10.1007/978-3319-77088-8_129-2

Bussmann RW, Batsatsashvili K, Kikvidze Z, Paniagua-Zambrana NY, Khutsishvili M, Maisaia I, Sikharulidze S, Tchelidze D. 2020. Sorbus aucuparia L. In: Batsatsashvili K, Kikvidze Z, Bussmann RW (Eds.) Ethnobotany of Mountain Regions Far Eastern Europe. Springer International Publishing, Cham. doi: 10.1007/978-3-319-77088-8_130-2

Bussmann RW, Batsatsashvili K, Kikvidze Z, Paniagua-Zambrana NY, Khutsishvili M, Maisaia I, Sikharulidze S, Tchelidze D. 2020. Swertia iberica Fisch. \& C.A. Mey. In: Batsatsashvili K, Kikvidze Z, Bussmann RW (Eds.) Ethnobotany of Mountain Regions Far Eastern Europe. Springer International Publishing, Cham. doi: 10.1007/978-3-319-770888_131-2

Bussmann RW, Batsatsashvili K, Kikvidze Z, Paniagua-Zambrana NY, Khutsishvili M, Maisaia I, Sikharulidze S, Tchelidze D. 2020. Tagetes erecta L. In: Batsatsashvili K, Kikvidze Z, Bussmann RW (Eds.) Ethnobotany of Mountain Regions Far Eastern Europe. Springer International Publishing, Cham. doi: 10.1007/978-3-319-77088-8_132-2

Bussmann RW, Batsatsashvili K, Kikvidze Z, Paniagua-Zambrana NY, Khutsishvili M, Maisaia I, Sikharulidze S, Tchelidze D. 2020. Tilia begonïfolia Steven, Tilia caucasica Rupr., Tilia cordata Mill. In: Batsatsashvili K, Kikvidze Z, Bussmann RW (Eds.) Ethnobotany of Mountain Regions Far Eastern Europe. Springer International Publishing, Cham. doi: 10.1007/978-3-319-77088-8_134-2

Bussmann RW, Batsatsashvili K, Kikvidze Z, Paniagua-Zambrana NY, Ghorbani A, Nasab Farzaneh K, Khutsishvili M, Maisaia I, Sikharulidze S, Tchelidze D. 2020. Tribulus terrestris L. In: Batsatsashvili K, Kikvidze Z, Bussmann RW (Eds.) Ethnobotany of Mountain Regions Far Eastern Europe. Springer International Publishing, Cham. doi: 10.1007/978-3319-77088-8_135-2

Bussmann RW, Batsatsashvili K, Kikvidze Z, Paniagua-Zambrana NY, Khutsishvili M, Maisaia I, Sikharulidze S, Tchelidze D. 2020. Triticum aestivum L., Triticum carthlicum Nevski. In: Batsatsashvili K, Kikvidze Z, Bussmann RW (Eds.) Ethnobotany of Mountain Regions Far Eastern Europe. Springer International Publishing, Cham. doi: 10.1007/978-3-319-77088-8_136-2

Bussmann RW, Batsatsashvili K, Kikvidze Z, Paniagua-Zambrana NY, Khutsishvili M, Maisaia I, Sikharulidze S, Tchelidze D. 2020. Valeriana officinalis L. In: Batsatsashvili K, Kikvidze Z, Bussmann RW (Eds.) Ethnobotany of Mountain Regions Far Eastern Europe. Springer International Publishing, Cham. doi: 10.1007/978-3-319-770888_139-2

Bussmann RW, Batsatsashvili K, Kikvidze Z, Paniagua-Zambrana NY, Khutsishvili M, Maisaia I, Sikharulidze S, Tchelidze D. 2020. Veratrum album L., Veratrum lobelianum Bernh. In: Batsatsashvili K, Kikvidze Z, Bussmann RW (Eds.) Ethnobotany of Mountain Regions Far Eastern Europe. Springer International Publishing, Cham. doi: 10.1007/978-3-319-77088-8_140-2 
Bussmann RW, Batsatsashvili K, Kikvidze Z, Paniagua-Zambrana NY, Khutsishvili M, Maisaia I, Sikharulidze S, Tchelidze D. 2020. Viscum album L. In: Batsatsashvili K, Kikvidze Z, Bussmann RW (Eds.) Ethnobotany of Mountain Regions Far Eastern Europe. Springer International Publishing, Cham. doi: 10.1007/978-3-319-77088-8_144-2

Bussmann RW, Batsatsashvili K, Kikvidze Z, Paniagua-Zambrana NY, Khutsishvili M, Maisaia I, Sikharulidze S, Tchelidze D. 2020. Vitis labrusca L., Vitis vinifera L. In: Batsatsashvili K, Kikvidze Z, Bussmann RW (Eds.) Ethnobotany of Mountain Regions Far Eastern Europe. Springer International Publishing, Cham. doi: 10.1007/978-3-319-770888_145-2

Bussmann RW, Batsatsashvili K, Kikvidze Z, Paniagua-Zambrana NY, Khutsishvili M, Maisaia I, Sikharulidze S, Tchelidze D. 2020. Zea mays L. In: Batsatsashvili K, Kikvidze Z, Bussmann RW (Eds.) Ethnobotany of Mountain Regions Far Eastern Europe. Springer International Publishing, Cham. doi: 10.1007/978-3-319-77088-8_146-2

Bussmann RW, Batsatsashvili K, Kikvidze Z, Paniagua-Zambrana NY, Ghorbani A, Nasab Farzaneh K, Khutsishvili M, Maisaia I, Sikharulidze S, Tchelidze D. 2020. Ziziphora clinopodioides Lam., Ziziphora serpyllacea Lam. In: Batsatsashvili K, Kikvidze Z, Bussmann RW (Eds.) Ethnobotany of Mountain Regions Far Eastern Europe. Springer International Publishing, Cham. doi: 10.1007/978-3-319-77088-8_147-2

Bussmann RW, Batsatsashvili K, Kikvidze Z, Paniagua-Zambrana NY, Khutsishvili M, Maisaia I, Sikharulidze S, Tchelidze D. 2020. Descurainia sophia (L.) Webb. ex. Prantl. In: Batsatsashvili K, Kikvidze Z, Bussmann RW (Eds.) Ethnobotany of Mountain Regions Far Eastern Europe. Springer International Publishing, Cham. doi: 10.1007/978-3319-77088-8_52-1

Bussmann RW, Batsatsashvili K, Kikvidze Z, Paniagua-Zambrana NY, Khutsishvili M, Maisaia I, Sikharulidze S, Tchelidze, D 2019. Bunias orientalis L. In: Batsatsashvili K, Kikvidze Z, Bussmann RW (Eds.) Ethnobotany of Mountain Regions Far Eastern Europe. Springer International Publishing, Cham. doi: 10.1007/978-3-319-77088-8_29-2

Bussmann RW, Batsatsashvili K, Kikvidze Z, Paniagua-Zambrana NY, Khutsishvili M, Maisaia I, Sikharulidze S, Tchelidze, D 2019. Matricaria chamomilla L. In: Batsatsashvili K, Kikvidze Z, Bussmann RW (Eds.) Ethnobotany of Mountain Regions Far Eastern Europe. Springer International Publishing, Cham.

Bussmann RW, Batsatsashvili K, Kikvidze Z, Paniagua-Zambrana NY, Khutsishvili M, Maisaia I, Sikharulidze S, Tchelidze, D 2019. Helleborus caucasicus A. Braun. In: Batsatsashvili K, Kikvidze Z, Bussmann RW (Eds.) Ethnobotany of Mountain Regions Far Eastern Europe. Springer International Publishing, Cham. doi: 10.1007/978-3-319-770888_70-2

Bussmann RW, Batsatsashvili K, Kikvidze Z, Paniagua-Zambrana NY, Khutsishvili M, Maisaia I, Sikharulidze S, Tchelidze, D 2019. Hordeum brevisubulatum subsp. violaceum (Boiss. \& Hohen.) Tzvelev, Hordeum vulgare L. In: Batsatsashvili K, Kikvidze Z, Bussmann RW (Eds.) Ethnobotany of Mountain Regions Far Eastern Europe. Springer International Publishing, Cham. doi: 10.1007/978-3-319-77088-8_72-2

Bussmann RW, Batsatsashvili K, Kikvidze Z, Paniagua-Zambrana NY, Khutsishvili M, Maisaia I, Sikharulidze S, Tchelidze, D 2019. Botrychium lunaria (L.) Sw. In: Batsatsashvili K, Kikvidze Z, Bussmann RW (Eds.) Ethnobotany of Mountain Regions Far Eastern Europe. Springer International Publishing, Cham. doi: 10.1007/978-3-319-770888_26-1

Bussmann RW, Batsatsashvili K, Kikvidze Z, Paniagua-Zambrana NY, Khutsishvili M, Maisaia I, Sikharulidze S, Tchelidze, D 2019. Brassica oleracea L. In: Batsatsashvili K, Kikvidze Z, Bussmann RW (Eds.) Ethnobotany of Mountain Regions Far Eastern Europe. Springer International Publishing, Cham. doi: 10.1007/978-3-319-77088-8_27-2

Bussmann RW, Batsatsashvili K, Kikvidze Z, Paniagua-Zambrana NY, Khutsishvili M, Maisaia I, Sikharulidze S, Tchelidze, D 2019. Cannabis sativa L. In: Batsatsashvili K, Kikvidze Z, Bussmann RW (Eds.) Ethnobotany of Mountain Regions Far Eastern Europe. Springer International Publishing, Cham. doi: 10.1007/978-3-319-77088-8_33-2

Bussmann RW, Batsatsashvili K, Kikvidze Z, Paniagua-Zambrana NY, Khutsishvili M, Maisaia I, Sikharulidze S, Tchelidze, D 2019. Cucurbita pepo L. In: Batsatsashvili K, Kikvidze Z, Bussmann RW (Eds.) Ethnobotany of Mountain Regions Far Eastern Europe. Springer International Publishing, Cham. doi: 10.1007/978-3-319-77088-8_48-2

Bussmann RW, Batsatsashvili K, Kikvidze Z, Paniagua-Zambrana NY, Moazzami Farida SH, Ghorbani A, Khutsishvili M, Maisaia I, Sikharulidze S, Tchelidze, D 2019. Dorema ammoniacum D. Don. In: Batsatsashvili K, Kikvidze Z, Bussmann RW (Eds.) Ethnobotany of Mountain Regions Far Eastern Europe. Springer International Publishing, Cham. doi: 10.1007/978-3-319-77088-8_53-1 
Bussmann RW, Batsatsashvili K, Kikvidze Z, Paniagua-Zambrana NY, Khutsishvili M, Maisaia I, Sikharulidze S, Tchelidze, D 2019. Nicotiana rustica L., Nicotiana tabacum L. In: Batsatsashvili K, Kikvidze Z, Bussmann RW (Eds.) Ethnobotany of Mountain Regions Far Eastern Europe. Springer International Publishing, Cham. doi: 10.1007/978-3319-77088-8_93-2

Bussmann RW, Batsatsashvili K, Kikvidze Z, Paniagua-Zambrana NY, Khutsishvili M, Maisaia I, Sikharulidze S, Tchelidze, D 2019. Oberna lacera Ikonn., Oberna wallichiana Ikonn. In: Batsatsashvili K, Kikvidze Z, Bussmann RW (Eds.) Ethnobotany of Mountain Regions Far Eastern Europe. Springer International Publishing, Cham. doi: 10.1007/978-3-319-77088-8_94-2

Bussmann RW, Batsatsashvili K, Kikvidze Z, Paniagua-Zambrana NY, Khutsishvili M, Maisaia I, Sikharulidze S, Tchelidze, D 2019. Ocimum basilicum L. In: Batsatsashvili K, Kikvidze Z, Bussmann RW (Eds.) Ethnobotany of Mountain Regions Far Eastern Europe. Springer International Publishing, Cham. doi: 10.1007/978-3-319-77088-8_95-2

Bussmann RW, Batsatsashvili K, Kikvidze Z, Paniagua-Zambrana NY, Khutsishvili M, Maisaia I, Sikharulidze S, Tchelidze, D 2019. Onobrychis viciifolia Scop. In: Batsatsashvili K, Kikvidze Z, Bussmann RW (Eds.) Ethnobotany of Mountain Regions Far Eastern Europe. Springer International Publishing, Cham. doi: 10.1007/978-3-319-770888_96-2

Bussmann RW, Batsatsashvili K, Kikvidze Z, Paniagua-Zambrana NY, Khutsishvili M, Maisaia I, Sikharulidze S, Tchelidze, D 2019. Persicaria maculosa Gray. In: Batsatsashvili K, Kikvidze Z, Bussmann RW (Eds.) Ethnobotany of Mountain Regions Far Eastern Europe. Springer International Publishing, Cham. doi: 10.1007/978-3-319-770888_100-2

Bussmann RW, Batsatsashvili K, Kikvidze Z, Paniagua-Zambrana NY, Moazzami Farida SH, Ghorbani A, Khutsishvili M, Maisaia I, Sikharulidze S, Tchelidze, D 2019. Pimpinella anisum L. In: Batsatsashvili K, Kikvidze Z, Bussmann RW (Eds.) Ethnobotany of Mountain Regions Far Eastern Europe. Springer International Publishing, Cham. doi: 10.1007/978-3-319-77088-8_105-2

Bussmann RW, Batsatsashvili K, Kikvidze Z, Paniagua-Zambrana NY, Khutsishvili M, Maisaia I, Sikharulidze S, Tchelidze, D 2019. Pisum sativum L. In: Batsatsashvili K, Kikvidze Z, Bussmann RW (Eds.) Ethnobotany of Mountain Regions Far Eastern Europe. Springer International Publishing, Cham. doi: 10.1007/978-3-319-77088-8_106-2

Bussmann RW, Batsatsashvili K, Kikvidze Z, Paniagua-Zambrana NY, Khutsishvili M, Maisaia I, Sikharulidze S, Tchelidze, D 2019. Pterocarya pterocarpa (Michx.) Kunth ex Iljinsk. In: Batsatsashvili K, Kikvidze Z, Bussmann RW (Eds.) Ethnobotany of Mountain Regions Far Eastern Europe. Springer International Publishing, Cham. doi: 10.1007/978-3319-77088-8_111-2

Bussmann RW, Batsatsashvili K, Kikvidze Z, Paniagua-Zambrana NY, Khutsishvili M, Maisaia I, Sikharulidze S, Tchelidze, D 2019. Cucumis sativus L. In: Batsatsashvili K, Kikvidze Z, Bussmann RW (Eds.) Ethnobotany of Mountain Regions Far Eastern Europe. Springer International Publishing, Cham. doi: 10.1007/978-3-319-77088-8_47-2

Bussmann RW, Batsatsashvili K, Kikvidze Z, Paniagua-Zambrana NY, Khutsishvili M, Maisaia I, Sikharulidze S, Tchelidze, D 2019. Cucumis melo L. In: Batsatsashvili K, Kikvidze Z, Bussmann RW (Eds.) Ethnobotany of Mountain Regions Far Eastern Europe. Springer International Publishing, Cham. doi: 10.1007/978-3-319-77088-8_46-1

Bussmann RW, Batsatsashvili K, Kikvidze Z, Paniagua-Zambrana NY, Khutsishvili M, Maisaia I, Sikharulidze S, Tchelidze, D 2019. Coronilla varia L. In: Batsatsashvili K, Kikvidze Z, Bussmann RW (Eds.) Ethnobotany of Mountain Regions Far Eastern Europe. Springer International Publishing, Cham. doi: 10.1007/978-3-319-77088-8_45-2

Bussmann RW, Batsatsashvili K, Kikvidze Z, Paniagua-Zambrana NY, Khutsishvili M, Maisaia I, Sikharulidze S, Tchelidze, D 2019. In: Batsatsashvili K, Kikvidze Z, Bussmann RW (Eds.) Ethnobotany of Mountain Regions Far Eastern Europe. Springer International Publishing, Cham.

Bussmann RW, Batsatsashvili K, Kikvidze Z, Paniagua-Zambrana NY, Khutsishvili M, Maisaia I, Sikharulidze S, Tchelidze, D 2019. Ethnobotany of Mountain Regions - Far Eastern Europe. In: Batsatsashvili K, Kikvidze Z, Bussmann RW (Eds.) Ethnobotany of Mountain Regions Far Eastern Europe. Springer International Publishing, Cham. doi: 10.1007/978-3-319-77088-8_1-1

Bussmann RW, Batsatsashvili K, Kikvidze Z, Paniagua-Zambrana NY, Khutsishvili M, Maisaia I, Sikharulidze S, Tchelidze, D 2019. Alnus barbata C.A. Mey., Alnus incana (L.) Moench. In: Batsatsashvili K, Kikvidze Z, Bussmann RW (Eds.) Ethnobotany of Mountain Regions Far Eastern Europe. Springer International Publishing, Cham. doi: 10.1007/978-3-319-77088-8_7-2 
Bussmann RW, Batsatsashvili K, Kikvidze Z, Paniagua-Zambrana NY, Moazzami Farida SH, Ghorbani A, Khutsishvili M, Maisaia I, Sikharulidze S, Tchelidze, D 2019. Anethum graveolens L. In: Batsatsashvili K, Kikvidze Z, Bussmann RW (Eds.) Ethnobotany of Mountain Regions Far Eastern Europe. Springer International Publishing, Cham. doi: 10.1007/978-3-319-77088-8_10-2

Bussmann RW, Batsatsashvili K, Kikvidze Z, Paniagua-Zambrana NY, Khutsishvili M, Maisaia I, Sikharulidze S, Tchelidze, D 2019. Anthriscus cerefolium (L.) Hoffm., Anthriscus sylvestris (L.) Hoffm. In: Batsatsashvili K, Kikvidze Z, Bussmann RW (Eds.) Ethnobotany of Mountain Regions Far Eastern Europe. Springer International Publishing, Cham. doi: 10.1007/978-3-319-77088-8_12-2

Bussmann RW, Batsatsashvili K, Kikvidze Z, Paniagua-Zambrana NY, Khutsishvili M, Maisaia I, Sikharulidze S, Tchelidze, D 2019. Armoracia rustica Schur. In: Batsatsashvili K, Kikvidze Z, Bussmann RW (Eds.) Ethnobotany of Mountain Regions Far Eastern Europe. Springer International Publishing, Cham. doi: 10.1007/978-3-319-770888_15-2

Bussmann RW, Batsatsashvili K, Kikvidze Z, Paniagua-Zambrana NY, Khutsishvili M, Maisaia I, Sikharulidze S, Tchelidze, D 2019. Arum italicum subsp. albispathum (Steven ex Ledeb.) Prime, Arum maculatum L. In: Batsatsashvili K, Kikvidze Z, Bussmann RW (Eds.) Ethnobotany of Mountain Regions Far Eastern Europe. Springer International Publishing, Cham. doi: 10.1007/978-3-319-77088-8_17-2

Bussmann RW, Batsatsashvili K, Kikvidze Z, Paniagua-Zambrana NY, Khutsishvili M, Maisaia I, Sikharulidze S, Tchelidze, D 2019. Astragalus caucasicus Pall. In: Batsatsashvili K, Kikvidze Z, Bussmann RW (Eds.) Ethnobotany of Mountain Regions Far Eastern Europe. Springer International Publishing, Cham. doi: 10.1007/978-3-319-770888_19-2

Bussmann RW, Batsatsashvili K, Kikvidze Z, Paniagua-Zambrana NY, Khutsishvili M, Maisaia I, Sikharulidze S, Tchelidze, D 2019. Astrantia maxima Pall. In: Batsatsashvili K, Kikvidze Z, Bussmann RW (Eds.) Ethnobotany of Mountain Regions Far Eastern Europe. Springer International Publishing, Cham. doi: 10.1007/978-3-319-770888_20-2

Bussmann RW, Batsatsashvili K, Kikvidze Z, Paniagua-Zambrana NY, Khutsishvili M, Maisaia I, Sikharulidze S, Tchelidze, D 2019. Avena sativa L. In: Batsatsashvili K, Kikvidze Z, Bussmann RW (Eds.) Ethnobotany of Mountain Regions Far Eastern Europe. Springer International Publishing, Cham. doi: 10.1007/978-3-319-77088-8_21-2

Bussmann RW, Batsatsashvili K, Kikvidze Z, Paniagua-Zambrana NY, Moazzami Farida SH, Ghorbani A, Khutsishvili M, Maisaia I, Sikharulidze S, Tchelidze, D 2019. Borago officinalis L. In: Batsatsashvili K, Kikvidze Z, Bussmann RW (Eds.) Ethnobotany of Mountain Regions Far Eastern Europe. Springer International Publishing, Cham. doi: 10.1007/978-3-319-77088-8_25-2

Bussmann RW, Batsatsashvili K, Kikvidze Z, Paniagua-Zambrana NY, Khutsishvili M, Maisaia I, Sikharulidze S, Tchelidze, D 2019. Capsicum annuum L. In: Batsatsashvili K, Kikvidze Z, Bussmann RW (Eds.) Ethnobotany of Mountain Regions Far Eastern Europe. Springer International Publishing, Cham. doi: 10.1007/978-3-319-77088-8_35-2

Bussmann RW, Batsatsashvili K, Kikvidze Z, Paniagua-Zambrana NY, Khutsishvili M, Maisaia I, Sikharulidze S, Tchelidze, D 2019. Cicer arietinum L. In: Batsatsashvili K, Kikvidze Z, Bussmann RW (Eds.) Ethnobotany of Mountain Regions Far Eastern Europe. Springer International Publishing, Cham. doi: 10.1007/978-3-319-77088-8_39-2

Bussmann RW, Batsatsashvili K, Kikvidze Z, Paniagua-Zambrana NY, Khutsishvili M, Maisaia I, Sikharulidze S, Tchelidze, D 2019. Clinopodium vulgare L. In: Batsatsashvili K, Kikvidze Z, Bussmann RW (Eds.) Ethnobotany of Mountain Regions Far Eastern Europe. Springer International Publishing, Cham. doi: 10.1007/978-3-319-770888_41-2

Bussmann RW, Batsatsashvili K, Kikvidze Z, Paniagua-Zambrana NY, Khutsishvili M, Maisaia I, Sikharulidze S, Tchelidze, D 2019. Cydonia oblonga Mill. In: Batsatsashvili K, Kikvidze Z, Bussmann RW (Eds.) Ethnobotany of Mountain Regions Far Eastern Europe. Springer International Publishing, Cham. doi: 10.1007/978-3-319-770888_49-2

Bussmann RW, Batsatsashvili K, Kikvidze Z, Paniagua-Zambrana NY, Khutsishvili M, Maisaia I, Sikharulidze S, Tchelidze, D 2019. Daphne caucasica Pall., Daphne glomerata Lam., Daphne mezereum L., Daphne pontica L. In: Batsatsashvili K, Kikvidze Z, Bussmann RW (Eds.) Ethnobotany of Mountain Regions Far Eastern Europe. Springer International Publishing, Cham. doi: 10.1007/978-3-319-77088-8_50-2 
Bussmann RW, Batsatsashvili K, Kikvidze Z, Paniagua-Zambrana NY, Moazzami Farida SH, Ghorbani A, Khutsishvili M, Maisaia I, Sikharulidze S, Tchelidze, D 2019. Daucus carota L. In: Batsatsashvili K, Kikvidze Z, Bussmann RW (Eds.) Ethnobotany of Mountain Regions Far Eastern Europe. Springer International Publishing, Cham. doi: 10.1007/978-3319-77088-8_51-2

Bussmann RW, Batsatsashvili K, Kikvidze Z, Paniagua-Zambrana NY, Khutsishvili M, Maisaia I, Sikharulidze S, Tchelidze, D 2019. Fagopyrum tataricum (L.) Gaertn. In: Batsatsashvili K, Kikvidze Z, Bussmann RW (Eds.) Ethnobotany of Mountain Regions Far Eastern Europe. Springer International Publishing, Cham. doi: 10.1007/978-3-319-770888_58-2

Bussmann RW, Batsatsashvili K, Kikvidze Z, Paniagua-Zambrana NY, Khutsishvili M, Maisaia I, Sikharulidze S, Tchelidze, D 2019. Ficus carica L. In: Batsatsashvili K, Kikvidze Z, Bussmann RW (Eds.) Ethnobotany of Mountain Regions Far Eastern Europe. Springer International Publishing, Cham. doi: 10.1007/978-3-319-77088-8_60-2

Bussmann RW, Batsatsashvili K, Kikvidze Z, Paniagua-Zambrana NY, Moazzami Farida SH, Ghorbani A, Khutsishvili M, Maisaia I, Sikharulidze S, Tchelidze, D 2019. Foeniculum vulgare Mill. In: Batsatsashvili K, Kikvidze Z, Bussmann RW (Eds.) Ethnobotany of Mountain Regions Far Eastern Europe. Springer International Publishing, Cham. doi: 10.1007/978-3-319-77088-8_61-2

Bussmann RW, Batsatsashvili K, Kikvidze Z, Paniagua-Zambrana NY, Khutsishvili M, Maisaia I, Sikharulidze S, Tchelidze, D 2019. Fraxinus excelsior L. In: Batsatsashvili K, Kikvidze Z, Bussmann RW (Eds.) Ethnobotany of Mountain Regions Far Eastern Europe. Springer International Publishing, Cham. doi: 10.1007/978-3-319-77088-8_62-2

Bussmann RW, Batsatsashvili K, Kikvidze Z, Paniagua-Zambrana NY, Khutsishvili M, Maisaia I, Sikharulidze S, Tchelidze, D 2019. Hyoscyamus nigerL. In: Batsatsashvili K, Kikvidze Z, Bussmann RW (Eds.) Ethnobotany of Mountain Regions Far Eastern Europe. Springer International Publishing, Cham. doi: 10.1007/978-3-319-77088-8_74-2

Bussmann RW, Batsatsashvili K, Kikvidze Z, Paniagua-Zambrana NY, Khutsishvili M, Maisaia I, Sikharulidze S, Tchelidze, D 2019. Isatis tinctoria L. In: Batsatsashvili K, Kikvidze Z, Bussmann RW (Eds.) Ethnobotany of Mountain Regions Far Eastern Europe. Springer International Publishing, Cham. doi: 10.1007/978-3-319-77088-8_76-2

Bussmann RW, Batsatsashvili K, Kikvidze Z, Paniagua-Zambrana NY, Khutsishvili M, Maisaia I, Sikharulidze S, Tchelidze, D 2019. Lapsana communis L., Lapsana grandiflora M. Bieb. In: Batsatsashvili K, Kikvidze Z, Bussmann RW (Eds.) Ethnobotany of Mountain Regions Far Eastern Europe. Springer International Publishing, Cham. doi: 10.1007/978-3-319-77088-8_79-2

Bussmann RW, Batsatsashvili K, Kikvidze Z, Paniagua-Zambrana NY, Khutsishvili M, Maisaia I, Sikharulidze S, Tchelidze, D 2019. Lepidium sativum L. In: Batsatsashvili K, Kikvidze Z, Bussmann RW (Eds.) Ethnobotany of Mountain Regions Far Eastern Europe. Springer International Publishing, Cham. doi: 10.1007/978-3-319-77088-8_81-2

Bussmann RW, Batsatsashvili K, Kikvidze Z, Paniagua-Zambrana NY, Khutsishvili M, Maisaia I, Sikharulidze S, Tchelidze, D 2019. Levisticum officinale W.D.J. Koch In: Batsatsashvili K, Kikvidze Z, Bussmann RW (Eds.) Ethnobotany of Mountain Regions Far Eastern Europe. Springer International Publishing, Cham. doi: 10.1007/978-3-319-770888_82-2

Bussmann RW, Batsatsashvili K, Kikvidze Z, Paniagua-Zambrana NY, Khutsishvili M, Maisaia I, Sikharulidze S, Tchelidze, D 2019. Linum usitatissimum L. In: Batsatsashvili K, Kikvidze Z, Bussmann RW (Eds.) Ethnobotany of Mountain Regions Far Eastern Europe. Springer International Publishing, Cham. doi: 10.1007/978-3-319-770888_84-2

Bussmann RW, Batsatsashvili K, Kikvidze Z, Paniagua-Zambrana NY, Khutsishvili M, Maisaia I, Sikharulidze S, Tchelidze, D 2019. Melissa offcinalis L. In: Batsatsashvili K, Kikvidze Z, Bussmann RW (Eds.) Ethnobotany of Mountain Regions Far Eastern Europe. Springer International Publishing, Cham. doi: 10.1007/978-3-319-77088-8_89-2

Bussmann RW, Batsatsashvili K, Kikvidze Z, Paniagua-Zambrana NY, Khutsishvili M, Maisaia I, Sikharulidze S, Tchelidze, D 2019. Petroselinum crispum (Mill.) Fuss. In: Batsatsashvili K, Kikvidze Z, Bussmann RW (Eds.) Ethnobotany of Mountain Regions Far Eastern Europe. Springer International Publishing, Cham. doi: 10.1007/978-3-319-770888_102-2

Bussmann RW, Batsatsashvili K, Kikvidze Z, Paniagua-Zambrana NY, Khutsishvili M, Maisaia I, Sikharulidze S, Tchelidze, D 2019. Polypodium vulgare L. In: Batsatsashvili K, Kikvidze Z, Bussmann RW (Eds.) Ethnobotany of Mountain Regions Far Eastern Europe. Springer International Publishing, Cham. doi: 10.1007/978-3-319-770888_109-2 
Bussmann RW, Batsatsashvili K, Kikvidze Z, Paniagua-Zambrana NY, Khutsishvili M, Maisaia I, Sikharulidze S, Tchelidze, D 2019. Taraxacum confusum Schischk., Taraxacum officinale F.H. Wigg. In: Batsatsashvili K, Kikvidze Z, Bussmann RW (Eds.) Ethnobotany of Mountain Regions Far Eastern Europe. Springer International Publishing, Cham. doi: 10.1007/978-3-319-77088-8_133-2

Bussmann RW, Batsatsashvili K, Kikvidze Z, Paniagua-Zambrana NY, Khutsishvili M, Maisaia I, Sikharulidze S, Tchelidze, D 2019. Centaurea behen L. In: Batsatsashvili K, Kikvidze Z, Bussmann RW (Eds.) Ethnobotany of Mountain Regions Far Eastern Europe. Springer International Publishing, Cham. doi: 10.1007/978-3-319-77088-8_37-1

Bussmann RW, Paniagua-Zambrana N.Y 2012. Traditional knowledge in a changing world - new insights from the Chacobo in Bolivia. In: Ponman B, Bussmann RW (eds.): Medicinal plants and the legacy of Richard E. Schultes. Proceedings of the Botany 2011 Richard E Schultes Symposium. William L. Brown Center, MBG, St. Louis, pp. 23-34.

Bussmann RW, Paniagua-Zambrana NY, Kunwar, RM. 2021. Fagara oxyphylla (Edgew.) Engl. In: Kunwar RW, Sher H, Bussmann RW. Ethnobotany of the Himalayas. Springer, Cham. doi: 10.1007/978-3-030-57408-6_100, pp. 927928.

Bussmann RW, Paniagua-Zambrana NY, Njoroge G, Grimshaw, J, 2021. Ensete ventricosum (Welw.) Cheesm. In: Bussmann RW, Ed. Ethnobotany of the Mountain Regions of Africa. Springer International Publishing: Cham. doi: 10.1007/978-3-319-77086-4_73-1, pp. 485-488.

Bussmann RW, Paniagua-Zambrana NY, Njoroge G, Grimshaw, J, 2021. Afrocarpus falcatus (Thunb.) C.N. Page, Afrocarpus gracilior (Pilg.) C.N. Page, Podocarpus milanjianus Rendle In: Bussmann RW, Ed. Ethnobotany of the Mountain Regions of Africa. Springer International Publishing: Cham. doi: 10.1007/978-3-319-77086-4_126_1

Bussmann RW, Paniagua-Zambrana NY, Njoroge G, Grimshaw, J, 2021. Rapanea melanophloeos (L.) Mez. In: Bussmann RW, Ed. Ethnobotany of the Mountain Regions of Africa. Springer International Publishing: Cham. doi: 10.1007/978-3-319-77086-4_133-1

Bussmann RW, Paniagua-Zambrana NY, Njoroge G, Grimshaw, J, 2021. Rumex abyssinicus Jacq., Rumex usambarensis (Dammer) Dammer In: Bussmann RW, Ed. Ethnobotany of the Mountain Regions of Africa. Springer International Publishing: Cham. doi: 10.1007/978-3-319-77086-4_138-1 pp. 919-926.

Bussmann RW, Paniagua-Zambrana NY, Njoroge G., Grimshaw, J, 2021. Hypericum revolutum Vahl In: Bussmann RW, Ed. Ethnobotany of the Mountain Regions of Africa. Springer International Publishing: Cham. doi: 10.1007/9783-030-38386-2_91, pp. 593-598.

Bussmann RW, Paniagua-Zambrana NY, Njoroge, G, 2021. Abutilon sp., Abutilon angulatum (Guill. \& Perr-.) Mast., Abutilon grandiflorum (Will.) Sweet., Abutilon longicuspe Hochst. ex A. Rich., Abutilon mauritianum (Jacq.) Medik., Hibiscus sp., Hibiscus calyphyllus Cav., Hibiscus vitifolius L. In: Bussmann RW, Ed. Ethnobotany of the Mountain Regions of Africa. Springer International Publishing: Cham. doi: 10.1007/978-3-319-77086-4_3-1, pp. 69-80.

Bussmann RW, Paniagua-Zambrana NY, Njoroge, G, 2021. Acacia drepanolobium Harmes ex Sjostedt, Acacia nilotica (L.) Willd. ex Delile, Acacia senegal (L.) Willd., Acacia seyal Delille, Acacia tortilis (Forssk.) Hayne In: Bussmann RW, Ed. Ethnobotany of the Mountain Regions of Africa. Springer International Publishing: Cham. doi: 10.1007/9783-319-77086-4_4-1, pp. 81-94.

Bussmann RW, Paniagua-Zambrana NY, Njoroge, G, 2021. Acanthus eminens C.B. Clarke, Acanthus sennii Chiov. In: Bussmann RW, Ed. Ethnobotany of the Mountain Regions of Africa. Springer International Publishing: Cham. doi: 10.1007/978-3-319-77086-4_5-1, pp. 95-100.

Bussmann RW, Paniagua-Zambrana NY, Njoroge, G, 2021. Achyranthes aspera L. In: Bussmann RW, Ed. Ethnobotany of the Mountain Regions of Africa. Springer International Publishing: Cham. doi: 10.1007/978-3-31977086-4_6-1, pp. 101-106.

Bussmann RW, Paniagua-Zambrana NY, Njoroge, G, 2021. Acokanthera schimperi (DC.) Schweinf. In: Bussmann RW, Ed. Ethnobotany of the Mountain Regions of Africa. Springer International Publishing: Cham. doi: 10.1007/9783-319-77086-4_7-1, pp. 107-110.

Bussmann RW, Paniagua-Zambrana NY, Njoroge, G, 2021. Adansonia digitata L. In: Bussmann RW, Ed. Ethnobotany of the Mountain Regions of Africa. Springer International Publishing: Cham. doi: 10.1007/978-3-319-77086-4_8-1, pp. 111-118. 
Bussmann RW, Paniagua-Zambrana NY, Njoroge, G, 2021. Aerva lanata (L.) Juss. ex Schult. In: Bussmann RW, Ed. Ethnobotany of the Mountain Regions of Africa. Springer International Publishing: Cham. doi: 10.1007/978-3-31977086-4_9-1, pp. 119-122.

Bussmann RW, Paniagua-Zambrana NY, Njoroge, G, 2021. Afrocanthium keniense (Bullock) Lantz, Afrocanthium lactescens (Hiern) Lantz, Canthium glaucum Hiern. In: Bussmann RW, Ed. Ethnobotany of the Mountain Regions of Africa. Springer International Publishing: Cham. doi: 10.1007/978-3-319-77086-4_10-1, pp. 123-130.

Bussmann RW, Paniagua-Zambrana NY, Njoroge, G, 2021. Ageratum conyzioides L. In: Bussmann RW, Ed. Ethnobotany of the Mountain Regions of Africa. Springer International Publishing: Cham. doi: 10.1007/978-3-31977086-4_14-1, pp. 131-134.

Bussmann RW, Paniagua-Zambrana NY, Njoroge, G, 2021. Albizia anthelminthica Brogn., Albizia gummifera (J.F. Gmel.) C.A. Sm. In: Bussmann RW, Ed. Ethnobotany of the Mountain Regions of Africa. Springer International Publishing: Cham. doi: 10.1007/978-3-319-77086-4_11-1, pp. 135-140.

Bussmann RW, Paniagua-Zambrana NY, Njoroge, G, 2021. Alchemilla argyrophylla Oliv., Alchemilla johnstonii Oliv. In: Bussmann RW, Ed. Ethnobotany of the Mountain Regions of Africa. Springer International Publishing: Cham. doi: 10.1007/978-3-319-77086-4_12_1, pp. 141-146.

Bussmann RW, Paniagua-Zambrana NY, Njoroge, G, 2021. Aloe sp., Aloe vera (L.) Burm. f. In: Bussmann RW, Ed. Ethnobotany of the Mountain Regions of Africa. Springer International Publishing: Cham. doi: 10.1007/978-3-31977086-4_13_1, pp. 147-152.

Bussmann RW, Paniagua-Zambrana NY, Njoroge, G, 2021. Amaranthus hybridus L., Amaranthus spinosus L. In: Bussmann RW, Ed. Ethnobotany of the Mountain Regions of Africa. Springer International Publishing: Cham. doi: 10.1007/978-3-319-77086-4_15_1, pp. 153-160.

Bussmann RW, Paniagua-Zambrana NY, Njoroge, G, 2021. Anthocleista zambesiaca Baker In: Bussmann RW, Ed. Ethnobotany of the Mountain Regions of Africa. Springer International Publishing: Cham. doi: 10.1007/978-3-31977086-4_16-1, pp. 161-164.

Bussmann RW, Paniagua-Zambrana NY, Njoroge, G, 2021. Artemisia afra Willd. In: Bussmann RW, Ed. Ethnobotany of the Mountain Regions of Africa. Springer International Publishing: Cham. doi: 10.1007/978-3-319-77086-4_17-1, pp. 165-172.

Bussmann RW, Paniagua-Zambrana NY, Njoroge, G, 2021. Asparagus africanus Lam., Asparagus falcatus L. In: Bussmann RW, Ed. Ethnobotany of the Mountain Regions of Africa. Springer International Publishing: Cham. doi: 10.1007/978-3-319-77086-4_18-1, pp. 173-180.

Bussmann RW, Paniagua-Zambrana NY, Njoroge, G, 2021. Asystasia gangetica (L.) T. Anderson In: Bussmann RW, Ed. Ethnobotany of the Mountain Regions of Africa. Springer International Publishing: Cham. doi: 10.1007/978-3319-77086-4_19-1, pp. 181-184.

Bussmann RW, Paniagua-Zambrana NY, Njoroge, G, 2021. Azadirachta indica A. Juss. In: Bussmann RW, Ed. Ethnobotany of the Mountain Regions of Africa. Springer International Publishing: Cham. doi: 10.1007/978-3-31977086-4_20-1, pp. 185-188.

Bussmann RW, Paniagua-Zambrana NY, Njoroge, G, 2021. Balanites aegyptiaca (L.) Delile, Balanites pedicellaris Mildbr. \& Schltr., Balanites rotundifolia (Tiegh.) Blatt. In: Bussmann RW, Ed. Ethnobotany of the Mountain Regions of Africa. Springer International Publishing: Cham. doi: 10.1007/978-3-319-77086-4_21-1, pp. 189-194.

Bussmann RW, Paniagua-Zambrana NY, Njoroge, G, 2021. Basella alba L. In: Bussmann RW, Ed. Ethnobotany of the Mountain Regions of Africa. Springer International Publishing: Cham. doi: 10.1007/978-3-319-77086-4_22-1, pp. 195-200.

Bussmann RW, Paniagua-Zambrana NY, Njoroge, G, 2021. Berberis holstii Engl. In: Bussmann RW, Ed. Ethnobotany of the Mountain Regions of Africa. Springer International Publishing: Cham. doi: 10.1007/978-3-319-77086-4_23_1, pp. 201-210.

Bussmann RW, Paniagua-Zambrana NY, Njoroge, G, 2021. Bersama abyssinica Fres. In: Bussmann RW, Ed. Ethnobotany of the Mountain Regions of Africa. Springer International Publishing: Cham. doi: 10.1007/978-3-31977086-4_24-1, pp. 211-216. 
Bussmann RW, Paniagua-Zambrana NY, Njoroge, G, 2021. Bidens pilosa L. In: Bussmann RW, Ed. Ethnobotany of the Mountain Regions of Africa. Springer International Publishing: Cham. doi: 10.1007/978-3-319-77086-4_25-1, pp. 217-220.

Bussmann RW, Paniagua-Zambrana NY, Njoroge, G, 2021. Blighia unïugata Bak. In: Bussmann RW, Ed. Ethnobotany of the Mountain Regions of Africa. Springer International Publishing: Cham. doi: 10.1007/978-3-31977086-4_36_1, pp. 221-222.

Bussmann RW, Paniagua-Zambrana NY, Njoroge, G, 2021. Bothriocline longipes (Oliv. \& Hiern.) N.E. Br. In: Bussmann RW, Ed. Ethnobotany of the Mountain Regions of Africa. Springer International Publishing: Cham. doi: 10.1007/978-3-319-77086-4_26-1, pp. 223-226.

Bussmann RW, Paniagua-Zambrana NY, Njoroge, G, 2021. Brachylaena huillensis O. Hoffm. In: Bussmann RW, Ed. Ethnobotany of the Mountain Regions of Africa. Springer International Publishing: Cham. doi: 10.1007/978-3-31977086-4_28-1, pp. 227-230.

Bussmann RW, Paniagua-Zambrana NY, Njoroge, G, 2021. Brucea antidysenterica J.F. Mill. In: Bussmann RW, Ed. Ethnobotany of the Mountain Regions of Africa. Springer International Publishing: Cham. doi: 10.1007/978-3-31977086-4_29-1, pp. 231-236.

Bussmann RW, Paniagua-Zambrana NY, Njoroge, G, 2021. Buddleja polystachya Fres. In: Bussmann RW, Ed. Ethnobotany of the Mountain Regions of Africa. Springer International Publishing: Cham. doi: 10.1007/978-3-31977086-4_30-1, pp. 237-242.

Bussmann RW, Paniagua-Zambrana NY, Njoroge, G, 2021. Caesalpinia volkensii Harms In: Bussmann RW, Ed. Ethnobotany of the Mountain Regions of Africa. Springer International Publishing: Cham. doi: 10.1007/978-3-31977086-4_31-1, pp. 243-248.

Bussmann RW, Paniagua-Zambrana NY, Njoroge, G, 2021. Calodendrum capense (L. f.) Thunb. In: Bussmann RW, Ed. Ethnobotany of the Mountain Regions of Africa. Springer International Publishing: Cham. doi: 10.1007/978-3319-77086-4_32-1, pp. 249-250.

Bussmann RW, Paniagua-Zambrana NY, Njoroge, G, 2021. Capparis fascicularis DC., Capparis tomentosa Lam., Cleome usambarica Pax, Gynandropsis gynandra (L.) Briq. In: Bussmann RW, Ed. Ethnobotany of the Mountain Regions of Africa. Springer International Publishing: Cham. doi: 10.1007/978-3-319-77086-4_33_1, pp. 251-260.

Bussmann RW, Paniagua-Zambrana NY, Njoroge, G, 2021. Carissa spinarum L. In: Bussmann RW, Ed. Ethnobotany of the Mountain Regions of Africa. Springer International Publishing: Cham. doi: 10.1007/978-3-319-77086-4_34-1, pp. 261-266.

Bussmann RW, Paniagua-Zambrana NY, Njoroge, G, 2021. Casearia battiscombei R.E. Fr. In: Bussmann RW, Ed. Ethnobotany of the Mountain Regions of Africa. Springer International Publishing: Cham. doi: 10.1007/978-3-31977086-4_35-1, pp. 267-268.

Bussmann RW, Paniagua-Zambrana NY, Njoroge, G, 2021. Cassipourea malosana (Baker) Alston In: Bussmann RW, Ed. Ethnobotany of the Mountain Regions of Africa. Springer International Publishing: Cham. doi: 10.1007/978-3319-77086-4_36-1, pp. 269-274.

Bussmann RW, Paniagua-Zambrana NY, Njoroge, G, 2021. Catha edulis Forssk. In: Bussmann RW, Ed. Ethnobotany of the Mountain Regions of Africa. Springer International Publishing: Cham. doi: 10.1007/978-3-319-77086-4_37-1, pp. 275-278.

Bussmann RW, Paniagua-Zambrana NY, Njoroge, G, 2021. Celtis africana Burm. f. In: Bussmann RW, Ed. Ethnobotany of the Mountain Regions of Africa. Springer International Publishing: Cham. doi: 10.1007/978-3-31977086-4_38-1, pp. 279-284.

Bussmann RW, Paniagua-Zambrana NY, Njoroge, G, 2021. Cissampelos pareira L. In: Bussmann RW, Ed. Ethnobotany of the Mountain Regions of Africa. Springer International Publishing: Cham. doi: 10.1007/978-3-31977086-4_39-1, pp. 285-288.

Bussmann RW, Paniagua-Zambrana NY, Njoroge, G, 2021. Clausena anisata (Willd.) Benth. In: Bussmann RW, Ed. Ethnobotany of the Mountain Regions of Africa. Springer International Publishing: Cham. doi: 10.1007/978-3-31977086-4_40-1, pp. 289-292. 
Bussmann RW, Paniagua-Zambrana NY, Njoroge, G, 2021. Clematis brachiata Thunb. In: Bussmann RW, Ed. Ethnobotany of the Mountain Regions of Africa. Springer International Publishing: Cham. doi: 10.1007/978-3-31977086-4_41-1, pp. 293-300.

Bussmann RW, Paniagua-Zambrana NY, Njoroge, G, 2021. Clerodendrum myricoides (Hochst.) R. Br. ex Vatke In: Bussmann RW, Ed. Ethnobotany of the Mountain Regions of Africa. Springer International Publishing: Cham. doi: 10.1007/978-3-319-77086-4_42-1, pp. 301-304.

Bussmann RW, Paniagua-Zambrana NY, Njoroge, G, 2021. Clutia abyssinica Jaub. \& Spach. In: Bussmann RW, Ed. Ethnobotany of the Mountain Regions of Africa. Springer International Publishing: Cham. doi: 10.1007/978-3-31977086-4_43-1, pp. 305-309.

Bussmann RW, Paniagua-Zambrana NY, Njoroge, G, 2021. Coccinia grandis (L.) Voigt In: Bussmann RW, Ed. Ethnobotany of the Mountain Regions of Africa. Springer International Publishing: Cham. doi: 10.1007/978-3-31977086-4_44-1, pp. 309-312.

Bussmann RW, Paniagua-Zambrana NY, Njoroge, G, 2021. Coffea arabica L. In: Bussmann RW, Ed. Ethnobotany of the Mountain Regions of Africa. Springer International Publishing: Cham. doi: 10.1007/978-3-319-77086-4_45-1, pp. 313-318.

Bussmann RW, Paniagua-Zambrana NY, Njoroge, G, 2021. Cola greenwayi Brenan In: Bussmann RW, Ed. Ethnobotany of the Mountain Regions of Africa. Springer International Publishing: Cham. doi: 10.1007/978-3-31977086-4_46-1, pp. 319-320.

Bussmann RW, Paniagua-Zambrana NY, Njoroge, G, 2021. Commelina africana L., Commelina benghalensis L., Commelina forskaolei Vahl In: Bussmann RW, Ed. Ethnobotany of the Mountain Regions of Africa. Springer International Publishing: Cham. doi: 10.1007/978-3-319-77086-4_47-1, pp. 321-330.

Bussmann RW, Paniagua-Zambrana NY, Njoroge, G, 2021. Commiphora africana (A. Rich.) Engl., Commiphora rostrata Engl., Commiphora schimperi (O. Bern.) Engl. In: Bussmann RW, Ed. Ethnobotany of the Mountain Regions of Africa. Springer International Publishing: Cham. doi: 10.1007/978-3-319-77086-4_48-1, pp. 331-336.

Bussmann RW, Paniagua-Zambrana NY, Njoroge, G, 2021. Conyza volkensii O. Hoffm. In: Bussmann RW, Ed. Ethnobotany of the Mountain Regions of Africa. Springer International Publishing: Cham. doi: 10.1007/978-3-31977086-4_49-1, pp. 337-340.

Bussmann RW, Paniagua-Zambrana NY, Njoroge, G, 2021. Cordia africana Lam., Cordia monoica Roxb., Cordia sinensis Lam. In: Bussmann RW, Ed. Ethnobotany of the Mountain Regions of Africa. Springer International Publishing: Cham. doi: 10.1007/978-3-319-77086-4_50-1, pp. 341-350.

Bussmann RW, Paniagua-Zambrana NY, Njoroge, G, 2021. Crotalaria agatiflora Schweinf., Crotalaria fascicularis Polhill, Crotalaria incana L., Crotalaria natalitia Meisn. In: Bussmann RW, Ed. Ethnobotany of the Mountain Regions of Africa. Springer International Publishing: Cham. doi: 10.1007/978-3-319-77086-4_51-1, pp. 351-356.

Bussmann RW, Paniagua-Zambrana NY, Njoroge, G, 2021. Croton dichogamus Pax, Croton megalocarpus Hutch., Steud. In: Bussmann RW, Ed. Ethnobotany of the Mountain Regions of Africa. Springer International Publishing: Cham. doi: 10.1007/978-3-319-77086-4_52-1, pp. 357-366

Bussmann RW, Paniagua-Zambrana NY, Njoroge, G, 2021. Cucumis dipsaceus Ehrenb. ex Spach In: Bussmann RW, Ed. Ethnobotany of the Mountain Regions of Africa. Springer International Publishing: Cham. doi: 10.1007/978-3319-77086-4_53-1, pp. 367-370.

Bussmann RW, Paniagua-Zambrana NY, Njoroge, G, 2021. Cupressus lusitanica Mill. In: Bussmann RW, Ed. Ethnobotany of the Mountain Regions of Africa. Springer International Publishing: Cham. doi: 10.1007/978-3-31977086-4_54-1, pp. 371-374.

Bussmann RW, Paniagua-Zambrana NY, Njoroge, G, 2021. Cussonia holstii Engl. In: Bussmann RW, Ed. Ethnobotany of the Mountain Regions of Africa. Springer International Publishing: Cham. doi: 10.1007/978-3-319-77086-4_55_1, pp. 375-378.

Bussmann RW, Paniagua-Zambrana NY, Njoroge, G, 2021. Cyathea manniana Hook. In: Bussmann RW, Ed. Ethnobotany of the Mountain Regions of Africa. Springer International Publishing: Cham. doi: 10.1007/978-3-31977086-4_56-1, pp. 379-384. 
Bussmann RW, Paniagua-Zambrana NY, Njoroge, G, 2021. Cyathula cylindrica Moq., Cyathula polycephala Bak. In: Bussmann RW, Ed. Ethnobotany of the Mountain Regions of Africa. Springer International Publishing: Cham. doi: 10.1007/978-3-319-77086-4_57-1, pp. 385-394.

Bussmann RW, Paniagua-Zambrana NY, Njoroge, G, 2021. Cynodon dactylon (L.) Pers. In: Bussmann RW, Ed. Ethnobotany of the Mountain Regions of Africa. Springer International Publishing: Cham. doi: 10.1007/978-3-31977086-4_58-1, pp. 395-398.

Bussmann RW, Paniagua-Zambrana NY, Njoroge, G, 2021. Cyphostemma maranguense (Gilg.) Desc. In: Bussmann RW, Ed. Ethnobotany of the Mountain Regions of Africa. Springer International Publishing: Cham. doi: 10.1007/9783-319-77086-4_59-1, pp. 399-402.

Bussmann RW, Paniagua-Zambrana NY, Njoroge, G, 2021. Dactyloctenium aegyticum (L.) Willd., Dactyloctenium giganteum B.S. Fischer \& Schweick. In: Bussmann RW, Ed. Ethnobotany of the Mountain Regions of Africa. Springer International Publishing: Cham. doi: 10.1007/978-3-319-77086-4_60-1, pp. 403-406.

Bussmann RW, Paniagua-Zambrana NY, Njoroge, G, 2021. Dalbergia lactea Vatke In: Bussmann RW, Ed. Ethnobotany of the Mountain Regions of Africa. Springer International Publishing: Cham. doi: 10.1007/978-3-31977086-4_61-1, pp. 407-410.

Bussmann RW, Paniagua-Zambrana NY, Njoroge, G, 2021. Datura stramonium L. In: Bussmann RW, Ed. Ethnobotany of the Mountain Regions of Africa. Springer International Publishing: Cham. doi: 10.1007/978-3-31977086-4_62-1, pp. 411-420.

Bussmann RW, Paniagua-Zambrana NY, Njoroge, G, 2021. Dioscorea dodecaneura Vell., Dioscorea dumetorum (Kunth) Pax, Dioscorea minutiflora Engl. In: Bussmann RW, Ed. Ethnobotany of the Mountain Regions of Africa. Springer International Publishing: Cham. doi: 10.1007/978-3-319-77086-4_63-1, pp. 421-428.

Bussmann RW, Paniagua-Zambrana NY, Njoroge, G, 2021. Diospyros abyssinica (Hiern.) F. White, Diospyros mespiliformis Hochst. ex A. DC. In: Bussmann RW, Ed. Ethnobotany of the Mountain Regions of Africa. Springer International Publishing: Cham. doi: 10.1007/978-3-319-77086-4_64-1, pp. 429-434.

Bussmann RW, Paniagua-Zambrana NY, Njoroge, G, 2021. Dodonaea viscosa Jacq. In: Bussmann RW, Ed. Ethnobotany of the Mountain Regions of Africa. Springer International Publishing: Cham. doi: 10.1007/978-3-31977086-4_65-1, pp. 435-440.

Bussmann RW, Paniagua-Zambrana NY, Njoroge, G, 2021. Dombeya sp., Dombeya torrida (J.F. Gmel.) Bamps In: Bussmann RW, Ed. Ethnobotany of the Mountain Regions of Africa. Springer International Publishing: Cham. doi: 10.1007/978-3-319-77086-4_66_1, pp. 441-442.

Bussmann RW, Paniagua-Zambrana NY, Njoroge, G, 2021. Dovyalis abyssinica (A. Rich.) Warb., Dovyalis caffra (Hook. f. \& Herv.) Warb., Dovyalis macrocalyx (Oliv.) Warb. In: Bussmann RW, Ed. Ethnobotany of the Mountain Regions of Africa. Springer International Publishing: Cham. doi: 10.1007/978-3-319-77086-4_66-1, pp. 453-460.

Bussmann RW, Paniagua-Zambrana NY, Njoroge, G, 2021. Dracaena afromontana Mildbr., Dracaena steudneri Engl. In: Bussmann RW, Ed. Ethnobotany of the Mountain Regions of Africa. Springer International Publishing: Cham. doi: 10.1007/978-3-319-77086-4_68-1, pp. 461-466.

Bussmann RW, Paniagua-Zambrana NY, Njoroge, G, 2021. Drypetes gerrardii Hutch. In: Bussmann RW, Ed. Ethnobotany of the Mountain Regions of Africa. Springer International Publishing: Cham. doi: 10.1007/978-3-31977086-4_69-1, pp. 467-468.

Bussmann RW, Paniagua-Zambrana NY, Njoroge, G, 2021. Ehretia cymosa Thonn. In: Bussmann RW, Ed. Ethnobotany of the Mountain Regions of Africa. Springer International Publishing: Cham. doi: 10.1007/978-3-31977086-4_70-1, pp. 469-472.

Bussmann RW, Paniagua-Zambrana NY, Njoroge, G, 2021. Ekebergia capensis Sparrm. In: Bussmann RW, Ed. Ethnobotany of the Mountain Regions of Africa. Springer International Publishing: Cham. doi: 10.1007/978-3-31977086-4_71-1, pp. 473-480.

Bussmann RW, Paniagua-Zambrana NY, Njoroge, G, 2021. Englerina woodfordioides (Schweinf.) Balle In: Bussmann RW, Ed. Ethnobotany of the Mountain Regions of Africa. Springer International Publishing: Cham. doi: 10.1007/9783-319-77086-4_72-1, pp. 481-484. 
Bussmann RW, Paniagua-Zambrana NY, Njoroge, G, 2021. Eragrostis tef (Zuccagni) Trotter In: Bussmann RW, Ed. Ethnobotany of the Mountain Regions of Africa. Springer International Publishing: Cham. doi: 10.1007/978-3-31977086-4_74_1, pp. 489-492.

Bussmann RW, Paniagua-Zambrana NY, Njoroge, G, 2021. Erica arborea L. In: Bussmann RW, Ed. Ethnobotany of the Mountain Regions of Africa. Springer International Publishing: Cham. doi: 10.1007/978-3-319-77086-4_75-1, pp. 493-500.

Bussmann RW, Paniagua-Zambrana NY, Njoroge, G, 2021. Erythrina abyssinica DC In: Bussmann RW, Ed. Ethnobotany of the Mountain Regions of Africa. Springer International Publishing: Cham. doi: 10.1007/978-3-31977086-4_76-1, pp. 501-506.

Bussmann RW, Paniagua-Zambrana NY, Njoroge, G, 2021. Erythrococca bongensis Pax In: Bussmann RW, Ed. Ethnobotany of the Mountain Regions of Africa. Springer International Publishing: Cham. doi: 10.1007/978-3-31977086-4_77-1, pp. 507-510.

Bussmann RW, Paniagua-Zambrana NY, Njoroge, G, 2021. Ethnobotany of Mountain Regions - African Mountains In: Bussmann RW, Ed. Ethnobotany of the Mountain Regions of Africa. Springer International Publishing: Cham. doi: 10.1007/978-3-319-77086-4_1-1, pp. 3-42.

Bussmann RW, Paniagua-Zambrana NY, Njoroge, G, 2021. Ethnobotany of mountain regions - Eastern Africa In: Bussmann RW, Ed. Ethnobotany of the Mountain Regions of Africa. Springer International Publishing: Cham. doi: 10.1007/978-3-319-77086-4_175-1, pp. 43-66.

Bussmann RW, Paniagua-Zambrana NY, Njoroge, G, 2021. Eucalyptus globulus Labil. In: Bussmann RW, Ed. Ethnobotany of the Mountain Regions of Africa. Springer International Publishing: Cham. doi: 10.1007/978-3-31977086-4_78-1, pp. 511-518.

Bussmann RW, Paniagua-Zambrana NY, Njoroge, G, 2021. Euclea divinorum Hiern In: Bussmann RW, Ed. Ethnobotany of the Mountain Regions of Africa. Springer International Publishing: Cham. doi: 10.1007/978-3-31977086-4_79-1, pp. 519-524.

Bussmann RW, Paniagua-Zambrana NY, Njoroge, G, 2021. Euphorbia candelabrum Kotsch, Euphorbia tiruncallii L. In: Bussmann RW, Ed. Ethnobotany of the Mountain Regions of Africa. Springer International Publishing: Cham. doi: 10.1007/978-3-319-77086-4_80-1, pp. 525-532.

Bussmann RW, Paniagua-Zambrana NY, Njoroge, G, 2021. Fagaropsis angolensis (Engl.) Dale In: Bussmann RW, Ed. Ethnobotany of the Mountain Regions of Africa. Springer International Publishing: Cham. doi: 10.1007/978-3319-77086-4_81-1, pp. 533-534.

Bussmann RW, Paniagua-Zambrana NY, Njoroge, G, 2021. Faurea saligna Harv. In: Bussmann RW, Ed. Ethnobotany of the Mountain Regions of Africa. Springer International Publishing: Cham. doi: 10.1007/978-3-319-77086-4_82-1, pp. 535-536.

Bussmann RW, Paniagua-Zambrana NY, Njoroge, G, 2021. Ficus sycomorus L., Ficus thonningii Blume In: Bussmann RW, Ed. Ethnobotany of the Mountain Regions of Africa. Springer International Publishing: Cham. doi: 10.1007/9783-319-77086-4_83-1, pp. 537-548.

Bussmann RW, Paniagua-Zambrana NY, Njoroge, G, 2021. Flacourtia indica (Burm. f.) Merr. In: Bussmann RW, Ed. Ethnobotany of the Mountain Regions of Africa. Springer International Publishing: Cham. doi: 10.1007/978-3-31977086-4_84-1, pp. 549-552.

Bussmann RW, Paniagua-Zambrana NY, Njoroge, G, 2021. Galinsoga parviflora Cav. In: Bussmann RW, Ed. Ethnobotany of the Mountain Regions of Africa. Springer International Publishing: Cham. doi: 10.1007/978-3-31977086-4_85-1, pp. 553-556.

Bussmann RW, Paniagua-Zambrana NY, Njoroge, G, 2021. Garcinia volkensii Engl. In: Bussmann RW, Ed. Ethnobotany of the Mountain Regions of Africa. Springer International Publishing: Cham. doi: 10.1007/978-3-31977086-4_86-1, pp. 557-560.

Bussmann RW, Paniagua-Zambrana NY, Njoroge, G, 2021. Gnidia glauca (Fres.) Gilg. In: Bussmann RW, Ed. Ethnobotany of the Mountain Regions of Africa. Springer International Publishing: Cham. doi: 10.1007/978-3-31977086-4_87-1, pp. 561-566. 
Bussmann RW, Paniagua-Zambrana NY, Njoroge, G, 2021. Grewia bicolor Juss., Grewia tembensis Fresen., Grewia tenax (Forssk.) Fiori, Grewia villosa Willd. In: Bussmann RW, Ed. Ethnobotany of the Mountain Regions of Africa. Springer International Publishing: Cham. doi: 10.1007/978-3-319-77086-4_88-1, pp. 567-576.

Bussmann RW, Paniagua-Zambrana NY, Njoroge, G, 2021. Hagenia abyssinica J.F. Gmel. In: Bussmann RW, Ed. Ethnobotany of the Mountain Regions of Africa. Springer International Publishing: Cham. doi: 10.1007/978-3-31977086-4_89-1, pp. 577-590.

Bussmann RW, Paniagua-Zambrana NY, Njoroge, G, 2021. Harungana madagascariensis Poir. In: Bussmann RW, Ed. Ethnobotany of the Mountain Regions of Africa. Springer International Publishing: Cham. doi: 10.1007/978-3319-77086-4_90-1, pp. 591-592.

Bussmann RW, Paniagua-Zambrana NY, Njoroge, G, 2021. Hypericum revolutum Vahl In: Bussmann RW, Ed. Ethnobotany of the Mountain Regions of Africa. Springer International Publishing: Cham. doi: 10.1007/978-3-31977086-4_91-1, pp. 593-598.

Bussmann RW, Paniagua-Zambrana NY, Njoroge, G, 2021. Hyphaene compressa H. Wendl., Hyphaene coriacea Gaertn., Hyphaene thebaica (L.) Mart. In: Bussmann RW, Ed. Ethnobotany of the Mountain Regions of Africa. Springer International Publishing: Cham. doi: 10.1007/978-3-319-77086-4_92-1, pp. 599-610.

Bussmann RW, Paniagua-Zambrana NY, Njoroge, G, 2021. Hypoestes aristata (Vahl) Rowm. \& Schult. In: Bussmann RW, Ed. Ethnobotany of the Mountain Regions of Africa. Springer International Publishing: Cham. doi: 10.1007/9783-319-77086-4_93-1, pp. 611-614.

Bussmann RW, Paniagua-Zambrana NY, Njoroge, G, 2021. /lex mitis (L.) Radkl. In: Bussmann RW, Ed. Ethnobotany of the Mountain Regions of Africa. Springer International Publishing: Cham. doi: 10.1007/978-3-319-77086-4_94-1, pp. 615-618.

Bussmann RW, Paniagua-Zambrana NY, Njoroge, G, 2021. Juniperus procera Hochst. ex Endl. In: Bussmann RW, Ed. Ethnobotany of the Mountain Regions of Africa. Springer International Publishing: Cham. doi: 10.1007/978-3319-77086-4_95_1, pp. 619-632.

Bussmann RW, Paniagua-Zambrana NY, Njoroge, G, 2021. Kalanchoe densiflora Rolfe, Kalanchoe petitiana A. Rich. In: Bussmann RW, Ed. Ethnobotany of the Mountain Regions of Africa. Springer International Publishing: Cham. doi: 10.1007/978-3-319-77086-4_96-1, pp. 633-640.

Bussmann RW, Paniagua-Zambrana NY, Njoroge, G, 2021. Kigelia africana (Lam.) Benth. In: Bussmann RW, Ed. Ethnobotany of the Mountain Regions of Africa. Springer International Publishing: Cham. doi: 10.1007/978-3-31977086-4_97_1, pp. 641-648.

Bussmann RW, Paniagua-Zambrana NY, Njoroge, G, 2021. Kyllingia bulbosa P. Beauv. In: Bussmann RW, Ed. Ethnobotany of the Mountain Regions of Africa. Springer International Publishing: Cham. doi: 10.1007/978-3-31977086-4_98-1, pp. 649-652.

Bussmann RW, Paniagua-Zambrana NY, Njoroge, G, 2021. Landolphia buchananii (Hallier f.) Stapf, Landolphia kirkii Dyler In: Bussmann RW, Ed. Ethnobotany of the Mountain Regions of Africa. Springer International Publishing: Cham. doi: Bussmann RW, Paniagua-Zambrana NY, Njoroge G, 10.1007/978-3-319-77086-4_99-1, pp. 653-656.

Bussmann RW, Paniagua-Zambrana NY, Njoroge, G, 2021. Lantana camara L. In: Bussmann RW, Ed. Ethnobotany of the Mountain Regions of Africa. Springer International Publishing: Cham. doi: 10.1007/978-3-319-77086-4_1001, pp. 657-662.

Bussmann RW, Paniagua-Zambrana NY, Njoroge, G, 2021. Lasianthus kilimandscharicus K. Schum. In: Bussmann RW, Ed. Ethnobotany of the Mountain Regions of Africa. Springer International Publishing: Cham. doi: 10.1007/9783-319-77086-4_101-1, pp. 663-668.

Bussmann RW, Paniagua-Zambrana NY, Njoroge, G, 2021. Lippia javanica (Burm. f.) Spreng. In: Bussmann RW, Ed. Ethnobotany of the Mountain Regions of Africa. Springer International Publishing: Cham. doi: 10.1007/978-3-31977086-4_102-1, pp. 667-672.

Bussmann RW, Paniagua-Zambrana NY, Njoroge, G, 2021. Lobelia gibberoa Hemsl. In: Bussmann RW, Ed. Ethnobotany of the Mountain Regions of Africa. Springer International Publishing: Cham. doi: 10.1007/978-3-31977086-4_103-1, pp. 673-683. 
Bussmann RW, Paniagua-Zambrana NY, Njoroge, G, 2021. Macaranga kilimandscharica Pax In: Bussmann RW, Ed. Ethnobotany of the Mountain Regions of Africa. Springer International Publishing: Cham. doi: 10.1007/978-3-31977086-4_104-1, pp. 677-684.

Bussmann RW, Paniagua-Zambrana NY, Njoroge, G, 2021. Maerua angolensis DC., Maerua triphylla A. Rich. In: Bussmann RW, Ed. Ethnobotany of the Mountain Regions of Africa. Springer International Publishing: Cham. doi: 10.1007/978-3-319-77086-4_105-1, pp. 685-690.

Bussmann RW, Paniagua-Zambrana NY, Njoroge, G, 2021. Mangifera indica L. In: Bussmann RW, Ed. Ethnobotany of the Mountain Regions of Africa. Springer International Publishing: Cham. doi: 10.1007/978-3-319-77086-4_1061, pp. 691-696.

Bussmann RW, Paniagua-Zambrana NY, Njoroge, G, 2021. Markhamia lutea (Benth.) K. Schum. In: Bussmann RW, Ed. Ethnobotany of the Mountain Regions of Africa. Springer International Publishing: Cham. doi: 10.1007/978-3319-77086-4_107-1, pp. 697-698.

Bussmann RW, Paniagua-Zambrana NY, Njoroge, G, 2021. Maytenus senegalensis (Lam.) Exell, Maytenus undata (Thunb.) Blakelock In: Bussmann RW, Ed. Ethnobotany of the Mountain Regions of Africa. Springer International Publishing: Cham. doi: 10.1007/978-3-319-77086-4_108-1, pp. 699-710.

Bussmann RW, Paniagua-Zambrana NY, Njoroge, G, 2021. Moringa oleifera Lam. In: Bussmann RW, Ed. Ethnobotany of the Mountain Regions of Africa. Springer International Publishing: Cham. doi: 10.1007/978-3-31977086-4_109-1, pp. 711-718.

Bussmann RW, Paniagua-Zambrana NY, Njoroge, G, 2021. Myrianthus holstii Engl. In: Bussmann RW, Ed. Ethnobotany of the Mountain Regions of Africa. Springer International Publishing: Cham. doi: 10.1007/978-3-31977086-4_110-1, pp. 717-720.

Bussmann RW, Paniagua-Zambrana NY, Njoroge, G, 2021. Myrsine africana L. In: Bussmann RW, Ed. Ethnobotany of the Mountain Regions of Africa. Springer International Publishing: Cham. doi: 10.1007/978-3-319-77086-4_1111, pp. 721-726.

Bussmann RW, Paniagua-Zambrana NY, Njoroge, G, 2021. Neoboutonia macrocalyx Pax In: Bussmann RW, Ed. Ethnobotany of the Mountain Regions of Africa. Springer International Publishing: Cham. doi: 10.1007/978-3-31977086-4_112-1, pp. 727-730.

Bussmann RW, Paniagua-Zambrana NY, Njoroge, G, 2021. Nicotiana rustica L., Nicotiana tabacum L. In: Bussmann RW, Ed. Ethnobotany of the Mountain Regions of Africa. Springer International Publishing: Cham. doi: 10.1007/9783-319-77086-4_113-1, pp. 731-738.

Bussmann RW, Paniagua-Zambrana NY, Njoroge, G, 2021. Nuxia congesta R. Br. ex Fresen. In: Bussmann RW, Ed. Ethnobotany of the Mountain Regions of Africa. Springer International Publishing: Cham. doi: 10.1007/978-3-31977086-4_114_1, pp. 739-744.

Bussmann RW, Paniagua-Zambrana NY, Njoroge, G, 2021. Ocimum gratissimum L., Ocimum kilimandscharicum Guerke In: Bussmann RW, Ed. Ethnobotany of the Mountain Regions of Africa. Springer International Publishing: Cham. doi: 10.1007/978-3-319-77086-4_116-1, pp. 745-752.

Bussmann RW, Paniagua-Zambrana NY, Njoroge, G, 2021. Ocotea usambarensis Engl. In: Bussmann RW, Ed. Ethnobotany of the Mountain Regions of Africa. Springer International Publishing: Cham. doi: 10.1007/978-3-31977086-4_115-1, pp. 753-760.

Bussmann RW, Paniagua-Zambrana NY, Njoroge, G, 2021. Oldeania alpina (K. Schum.) Stapleton In: Bussmann RW, Ed. Ethnobotany of the Mountain Regions of Africa. Springer International Publishing: Cham. doi: 10.1007/978-3319-77086-4_176-1, pp. 761-776.

Bussmann RW, Paniagua-Zambrana NY, Njoroge, G, 2021. Olea capensis L., Olea europaea subsp. africana (Mill.) P.S. Greene In: Bussmann RW, Ed. Ethnobotany of the Mountain Regions of Africa. Springer International Publishing: Cham. doi: 10.1007/978-3-319-77086-4_117-1, pp. 777-786.

Bussmann RW, Paniagua-Zambrana NY, Njoroge, G, 2021. Osyris lanceolata Hochst. \& Steud. In: Bussmann RW, Ed. Ethnobotany of the Mountain Regions of Africa. Springer International Publishing: Cham. doi: 10.1007/978-3319-77086-4_118-1, pp. 787-790. 
Bussmann RW, Paniagua-Zambrana NY, Njoroge, G, 2021. Pavonia urens Cav. In: Bussmann RW, Ed. Ethnobotany of the Mountain Regions of Africa. Springer International Publishing: Cham. doi: 10.1007/978-3-319-77086-4_1191, pp. 791-794.

Bussmann RW, Paniagua-Zambrana NY, Njoroge, G, 2021. Phoenix reclinata Jacq. In: Bussmann RW, Ed. Ethnobotany of the Mountain Regions of Africa. Springer International Publishing: Cham. doi: 10.1007/978-3-31977086-4_120-1, pp. 795-800.

Bussmann RW, Paniagua-Zambrana NY, Njoroge, G, 2021. Physalis peruviana L. In: Bussmann RW, Ed. Ethnobotany of the Mountain Regions of Africa. Springer International Publishing: Cham. doi: 10.1007/978-3-319-77086-4_1211, pp. 801-806.

Bussmann RW, Paniagua-Zambrana NY, Njoroge, G, 2021. Phytolacca dodecandra L'Hér. In: Bussmann RW, Ed. Ethnobotany of the Mountain Regions of Africa. Springer International Publishing: Cham. doi: 10.1007/978-3-31977086-4_122-1, pp. 807-814.

Bussmann RW, Paniagua-Zambrana NY, Njoroge, G, 2021. Pinus patula Schlect. \& Cham., Pinus radiata D. Don. In: Bussmann RW, Ed. Ethnobotany of the Mountain Regions of Africa. Springer International Publishing: Cham. doi: 10.1007/978-3-319-77086-4_124-1, pp. 815-824.

Bussmann RW, Paniagua-Zambrana NY, Njoroge, G, 2021. Piper capense L. In: Bussmann RW, Ed. Ethnobotany of the Mountain Regions of Africa. Springer International Publishing: Cham. doi: 10.1007/978-3-319-77086-4_123-1, pp. 825-830.

Bussmann RW, Paniagua-Zambrana NY, Njoroge, G, 2021. Pistacia aethiopica Kokwaro In: Bussmann RW, Ed. Ethnobotany of the Mountain Regions of Africa. Springer International Publishing: Cham. doi: 10.1007/978-3-31977086-4_125-1, pp. 831-834.

Bussmann RW, Paniagua-Zambrana NY, Njoroge, G, 2021. Portulaca oleracea L. In: Bussmann RW, Ed. Ethnobotany of the Mountain Regions of Africa. Springer International Publishing: Cham. doi: 10.1007/978-3-319-77086-4_1271, pp. 845-850.

Bussmann RW, Paniagua-Zambrana NY, Njoroge, G, 2021. Pouteria adolfi-friederici (Engl.) A. Meeuse, Pouteria altissima (A. Chev.) Baehni In: Bussmann RW, Ed. Ethnobotany of the Mountain Regions of Africa. Springer International Publishing: Cham. doi: 10.1007/978-3-319-77086-4_128-1, pp. 851-856.

Bussmann RW, Paniagua-Zambrana NY, Njoroge, G, 2021. Prunus africana (Hook. f.) Kalkman In: Bussmann RW, Ed. Ethnobotany of the Mountain Regions of Africa. Springer International Publishing: Cham. doi: 10.1007/978-3319-77086-4_129-1, pp. 857-862.

Bussmann RW, Paniagua-Zambrana NY, Njoroge, G, 2021. Psidium guajava L. In: Bussmann RW, Ed. Ethnobotany of the Mountain Regions of Africa. Springer International Publishing: Cham. doi: 10.1007/978-3-319-77086-4_1301, pp. 863-868.

Bussmann RW, Paniagua-Zambrana NY, Njoroge, G, 2021. Psychotria orophila E.A.A. Petit, Psychotria sp. In: Bussmann RW, Ed. Ethnobotany of the Mountain Regions of Africa. Springer International Publishing: Cham. doi: 10.1007/978-3-319-77086-4_131-1, pp. 869-872.

Bussmann RW, Paniagua-Zambrana NY, Njoroge, G, 2021. Pterolobium stellatum (Forssk.) Brenan In: Bussmann RW, Ed. Ethnobotany of the Mountain Regions of Africa. Springer International Publishing: Cham. doi: 10.1007/9783-319-77086-4_132-1, pp. 873-878.

Bussmann RW, Paniagua-Zambrana NY, Njoroge, G, 2021. Rhamnus prinoides L'Hér., Rhamnus staddo A. Rich. In: Bussmann RW, Ed. Ethnobotany of the Mountain Regions of Africa. Springer International Publishing: Cham. doi: 10.1007/978-3-319-77086-4_134-1, pp. 885-892.

Bussmann RW, Paniagua-Zambrana NY, Njoroge, G, 2021. Rhus natalensis Bernh. ex C. Krauss, Rhus ruspolii Engl., Rhus tenuinervis Engl., Rhus vulgaris Meikle In: Bussmann RW, Ed. Ethnobotany of the Mountain Regions of Africa. Springer International Publishing: Cham. doi: 10.1007/978-3-319-77086-4_135-1, pp. 893-902.

Bussmann RW, Paniagua-Zambrana NY, Njoroge, G, 2021. Rich., Vigna subterranea (L.) Verdc., Vigna unguiculata (L.) Walp. In: Bussmann RW, Ed. Ethnobotany of the Mountain Regions of Africa. Springer International Publishing: Cham. doi: 10.1007/978-3-319-77086-4_167-1, pp. 1079-1084. 
Bussmann RW, Paniagua-Zambrana NY, Njoroge, G, 2021. Ricinus communis L. In: Bussmann RW, Ed. Ethnobotany of the Mountain Regions of Africa. Springer International Publishing: Cham. doi: 10.1007/978-3-319-77086-4_1361, pp. 903-910.

Bussmann RW, Paniagua-Zambrana NY, Njoroge, G, 2021. Rubus apetalus Poir., Rubus rosifolius Sm., Rubus volkensii Engl. In: Bussmann RW, Ed. Ethnobotany of the Mountain Regions of Africa. Springer International Publishing: Cham. doi: 10.1007/978-3-319-77086-4_137-1, pp. 911-918.

Bussmann RW, Paniagua-Zambrana NY, Njoroge, G, 2021. Salvadora persica L. In: Bussmann RW, Ed. Ethnobotany of the Mountain Regions of Africa. Springer International Publishing: Cham. doi: 10.1007/978-3-319-77086-4_1391, pp. 927-930.

Bussmann RW, Paniagua-Zambrana NY, Njoroge, G, 2021. Sambucus africana Engl. In: Bussmann RW, Ed. Ethnobotany of the Mountain Regions of Africa. Springer International Publishing: Cham. doi: 10.1007/978-3-31977086-4_140-1, pp. 931-943.

Bussmann RW, Paniagua-Zambrana NY, Njoroge, G, 2021. Schefflera volkensii (Harms) Harms In: Bussmann RW, Ed. Ethnobotany of the Mountain Regions of Africa. Springer International Publishing: Cham. doi: 10.1007/978-3319-77086-4_142_1, pp. 943-948.

Bussmann RW, Paniagua-Zambrana NY, Njoroge, G, 2021. Schkuhria pinnata (Lam.) Thell. In: Bussmann RW, Ed. Ethnobotany of the Mountain Regions of Africa. Springer International Publishing: Cham. doi: 10.1007/978-3-31977086-4_141_1 pp. 949-952.

Bussmann RW, Paniagua-Zambrana NY, Njoroge, G, 2021. Scutia myrtina (Burm. f.) Kurz In: Bussmann RW, Ed. Ethnobotany of the Mountain Regions of Africa. Springer International Publishing: Cham. doi: 10.1007/978-3-31977086-4_143_1, pp. 953-958.

Bussmann RW, Paniagua-Zambrana NY, Njoroge, G, 2021. Solanecio mannii (Hook. f.) C. Jeffrey In: Bussmann RW, Ed. Ethnobotany of the Mountain Regions of Africa. Springer International Publishing: Cham. doi: 10.1007/978-3319-77086-4_145_1, pp. 959-962.

Bussmann RW, Paniagua-Zambrana NY, Njoroge, G, 2021. Solanum aculeastrum (Gilg.) Desc., Solanum anguivi Lam., Solanum incanum L., Solanum indicum L., Solanum nigrum L. In: Bussmann RW, Ed. Ethnobotany of the Mountain Regions of Africa. Springer International Publishing: Cham. doi: 10.1007/978-3-319-77086-4_146_1, pp. 963-974.

Bussmann RW, Paniagua-Zambrana NY, Njoroge, G, 2021. Sonchus asper L, Sonchus oleraceus L. In: Bussmann RW, Ed. Ethnobotany of the Mountain Regions of Africa. Springer International Publishing: Cham. doi: 10.1007/9783-319-77086-4_147_1, pp. 975-982.

Bussmann RW, Paniagua-Zambrana NY, Njoroge, G, 2021. Spilanthes mauritiana (Pers.) DC. In: Bussmann RW, Ed. Ethnobotany of the Mountain Regions of Africa. Springer International Publishing: Cham. doi: 10.1007/978-3-31977086-4_148-1, pp. 983-984.

Bussmann RW, Paniagua-Zambrana NY, Njoroge, G, 2021. Stephania abyssinica (Dillon \& A. Rich.) Walp. In: Bussmann RW, Ed. Ethnobotany of the Mountain Regions of Africa. Springer International Publishing: Cham. doi: 10.1007/978-3-319-77086-4_149_1, pp. 985-988.

Bussmann RW, Paniagua-Zambrana NY, Njoroge, G, 2021. Stoebe kilimandscharica O. Hoffm. In: Bussmann RW, Ed. Ethnobotany of the Mountain Regions of Africa. Springer International Publishing: Cham. doi: 10.1007/978-3319-77086-4_150-1, pp. 989-990.

Bussmann RW, Paniagua-Zambrana NY, Njoroge, G, 2021. Strychnos henningsii Gilg., Strychnos spinosa Lam., Strychnos usambarensis Gilg. In: Bussmann RW, Ed. Ethnobotany of the Mountain Regions of Africa. Springer International Publishing: Cham. doi: 10.1007/978-3-319-77086-4_151-1, pp. 991-996.

Bussmann RW, Paniagua-Zambrana NY, Njoroge, G, 2021. Syzygium cordatum Hochst., Syzygium guineense (Willd.) DC. In: Bussmann RW, Ed. Ethnobotany of the Mountain Regions of Africa. Springer International Publishing: Cham. doi: 10.1007/978-3-319-77086-4_152_1, pp. 997-1004. 
Bussmann RW, Paniagua-Zambrana NY, Njoroge, G, 2021. Tabernaemontana stapfiana Brizten In: Bussmann RW, Ed. Ethnobotany of the Mountain Regions of Africa. Springer International Publishing: Cham. doi: 10.1007/978-3319-77086-4_153_1, pp. 1005-1008.

Bussmann RW, Paniagua-Zambrana NY, Njoroge, G, 2021. Tagetes minuta L. In: Bussmann RW, Ed. Ethnobotany of the Mountain Regions of Africa. Springer International Publishing: Cham. doi: 10.1007/978-3-319-77086-4_156_1, pp. 1009-1012.

Bussmann RW, Paniagua-Zambrana NY, Njoroge, G, 2021. Tamarindus indica L. In: Bussmann RW, Ed. Ethnobotany of the Mountain Regions of Africa. Springer International Publishing: Cham. doi: 10.1007/978-3-319-77086-4_154_1, pp. 1013-1016.

Bussmann RW, Paniagua-Zambrana NY, Njoroge, G, 2021. Tarchonanthus camphoratus L. In: Bussmann RW, Ed. Ethnobotany of the Mountain Regions of Africa. Springer International Publishing: Cham. doi: 10.1007/978-3-31977086-4_155_1, pp. 1017-1020.

Bussmann RW, Paniagua-Zambrana NY, Njoroge, G, 2021. Teclea simplicifolia Engl., Vepris dainelii (Pic. Serm.) Mziray, Vepris nobilis Delile. In: Bussmann RW, Ed. Ethnobotany of the Mountain Regions of Africa. Springer International Publishing: Cham. doi: 10.1007/978-3-319-77086-4_160-1, pp. 1021-1030.

Bussmann RW, Paniagua-Zambrana NY, Njoroge, G, 2021. Themeda triandra Forssk. In: Bussmann RW, Ed. Ethnobotany of the Mountain Regions of Africa. Springer International Publishing: Cham. doi: 10.1007/978-3-31977086-4_157-1, pp. 1031-1032.

Bussmann RW, Paniagua-Zambrana NY, Njoroge, G, 2021. Thunbergia alata Sims In: Bussmann RW, Ed. Ethnobotany of the Mountain Regions of Africa. Springer International Publishing: Cham. doi: 10.1007/978-3-31977086-4_158_1, pp. 1033-1036.

Bussmann RW, Paniagua-Zambrana NY, Njoroge, G, 2021. Toddalia asiatica (L.) Lam. In: Bussmann RW, Ed. Ethnobotany of the Mountain Regions of Africa. Springer International Publishing: Cham. doi: 10.1007/978-3-31977086-4_159_1, pp. 1037-1040.

Bussmann RW, Paniagua-Zambrana NY, Njoroge, G, 2021. Trichilia emeticaVahl In: Bussmann RW, Ed. Ethnobotany of the Mountain Regions of Africa. Springer International Publishing: Cham. doi: 10.1007/978-3-319-77086-4_1611, pp. 1041-1044.

Bussmann RW, Paniagua-Zambrana NY, Njoroge, G, 2021. Typha domingensis Pers., Typha latifolia L. In: Bussmann RW, Ed. Ethnobotany of the Mountain Regions of Africa. Springer International Publishing: Cham. doi: 10.1007/9783-319-77086-4_162-1, pp. 1045-1052.

Bussmann RW, Paniagua-Zambrana NY, Njoroge, G, 2021. Urtica massaica Mildbr. In: Bussmann RW, Ed. Ethnobotany of the Mountain Regions of Africa. Springer International Publishing: Cham. doi: 10.1007/978-3-31977086-4_163-1, pp. 1053-1058.

Bussmann RW, Paniagua-Zambrana NY, Njoroge, G, 2021. Uvaria acuminata Oliv., Uvaria scheffleri L.L. Zhou, Y.C.F. Su \& R.M.K Saunders In: Bussmann RW, Ed. Ethnobotany of the Mountain Regions of Africa. Springer International Publishing: Cham. doi: 10.1007/978-3-319-77086-4_164-1, pp. 1059-1062.

Bussmann RW, Paniagua-Zambrana NY, Njoroge, G, 2021. Vangueria apiculata K. Schum., Vangueria infausta Burch., Vangueria madagascariensis J.F. Gmel., Vangueria volkensii K. Schum. In: Bussmann RW, Ed. Ethnobotany of the Mountain Regions of Africa. Springer International Publishing: Cham. doi: 10.1007/978-3-319-77086-4_165-1, pp. 1063-1068.

Bussmann RW, Paniagua-Zambrana NY, Njoroge, G, 2021. Vernonia amygdalina Deille, Vernionia auriculifera Hiern, Vernionia galamensis (Cass.) Less., Vernonia lasiopus O. Hoffm. In: Bussmann RW, Ed. Ethnobotany of the Mountain Regions of Africa. Springer International Publishing: Cham. doi: 10.1007/978-3-319-77086-4_166-1, pp. 1069-1078.

Bussmann RW, Paniagua-Zambrana NY, Njoroge, G, 2021. Vitex keniensis Turrill In: Bussmann RW, Ed. Ethnobotany of the Mountain Regions of Africa. Springer International Publishing: Cham. doi: 10.1007/978-3-319-77086-4_1681, pp. 1085-1091. 
Bussmann RW, Paniagua-Zambrana NY, Njoroge, G, 2021. Warburgia salutaris (Bertol.) Chiov. In: Bussmann RW, Ed. Ethnobotany of the Mountain Regions of Africa. Springer International Publishing: Cham. doi: 10.1007/978-3319-77086-4_169-1, pp. 1089-1092.

Bussmann RW, Paniagua-Zambrana NY, Njoroge, G, 2021. Withania somnifera (L.) Dunal In: Bussmann RW, Ed. Ethnobotany of the Mountain Regions of Africa. Springer International Publishing: Cham. doi: 10.1007/978-3-31977086-4_170-1, pp. 1093-1098.

Bussmann RW, Paniagua-Zambrana NY, Njoroge, G, 2021. Ximenia americana L. In: Bussmann RW, Ed. Ethnobotany of the Mountain Regions of Africa. Springer International Publishing: Cham. doi: 10.1007/978-3-319-77086-4_1711, pp. 1099-1109.

Bussmann RW, Paniagua-Zambrana NY, Njoroge, G, 2021. Zanthoxylum chalybaeum Engl., Zanthoxylum gillettii (De Wild) Waterm., Zanthoxylum usambarense (Engl.) Kokwaro In: Bussmann RW, Ed. Ethnobotany of the Mountain Regions of Africa. Springer International Publishing: Cham. doi: 10.1007/978-3-319-77086-4_172-1, pp. 1103-1110.

Bussmann RW, Paniagua-Zambrana NY, Njoroge, G, 2021. Zehneria scabra (L.f.) Spond. In: Bussmann RW, Ed. Ethnobotany of the Mountain Regions of Africa. Springer International Publishing: Cham. doi: 10.1007/978-3-31977086-4_173-1, , pp. 1111-1124.

Bussmann RW, Paniagua-Zambrana NY, Njoroge, G, Ali M, Jan, HA 2021. Ziziphus mauritiana Lam., Ziziphus mucronata Willd. In: Bussmann RW, Ed. Ethnobotany of the Mountain Regions of Africa. Springer International Publishing: Cham. doi: 10.1007/978-3-319-77086-4_174-1, pp. 1117-1125.

Bussmann RW, Paniagua-Zambrana NY. 2021. Carum carvi L., Carum copticum (L.) Benth. \& Hook. f. In: Kunwar RW, Sher H, Bussmann RW. Ethnobotany of the Himalayas. Springer, Cham. doi: 10.1007/978-3-030-57408-6_48, pp. 469-476

Bussmann RW, Paniagua-Zambrana NY. 2021. Curculigo orchioides Benth. In: Kunwar RW, Sher H, Bussmann RW. Ethnobotany of the Himalayas. Springer, Cham. doi: 10.1007/978-3-030-57408-6_69, pp. 643-648.

Bussmann RW, Paniagua-Zambrana NY. 2021. Seseli libanotis (L.) W. Koch, Seseli transcaucasicum Pimenov \& Sdobina. In: Kunwar RW, Sher H, Bussmann RW. Ethnobotany of the Himalayas. Springer, Cham. doi: 10.1007/9783-030-57408-6_226, pp. 1855-1860.

Bussmann RW, Paniagua-Zambrana NY, Sikharulidze S, Kikvidze Z, Kikodze D, Tchelidze D, Batsatsashvili K 2020. Ethnobotany of the Silk Road - Georgia, the Cradle of Wine. In: Cooper,R, Deakin JJ (Eds). Natural Products of Silk Road Plants. CRC Press, Bioca Raton.

Cámara-Leret R, Paniagua-Zambrana NY, Macía MJ. 2012. Standard Protocol for Gathering Palm Ethnobotanical Data and Socioeconomic Variables across the Tropics. In: Ponman B, Bussmann RW (eds.): Medicinal plants and the legacy of Richard E. Schultes. Proceedings of the Botany 2011 Richard E Schultes Symposium. Graficart, Trujillo, pp. 41-72.

Charmakar S, Kunwar RM, Kafle MN, Pradhan DK, Jan HA, Abbasi AM, Bussmann RW, Paniagua-Zambrana NY. 2021. Daphne bholua Buch.-Ham. ex D. Don., Daphne mucronata Royle, Daphne papyrica Wall. ex G. Don. In: Kunwar RW, Sher H, Bussmann RW. Ethnobotany of the Himalayas. Springer, Cham. doi: 10.1007/978-3-030-57408-6_76, pp. 705724.

Chaudhary CM, Jan HA, Kunwar RM, Bussmann RW, Paniagua-Zambrana NY 2021. Caesalpinia decapetala (Roith) Alston Fabaceae In: Kunwar RW, Sher H, Bussmann RW. Ethnobotany of the Himalayas. Springer, Cham. doi: 10.1007/978-3-030-57408-6_43, pp. 431-438.

da Costa Ferreira E, Paniagua-Zambrana NY, Bussmann RW, Paiva de Lucena RF, Dias da Cruz D. 2019. Perfil dos vendedores de plantas medicinais em mercados públicos na Paraíba, Nordeste, Brasil. In: Dias da Cruz, D, Cavalcanti de Miranda, GE, Israel de Souza, B, Paiva de Lucena, RF. Meio ambiente e desenvolvimiento - os desafios da sustentabilidade ambiental. UFPB, Joao Pessoa, 13-20.

Dahal N, Mahat R, Thapa A, Kunwar RM, Bussmann RW, Paniagua-Zambrana NY, Ali M, Hussain W. 2021. Scutellaria barbata D. Don, Scutellaria discolor Colebr., Scutellaria orientalis L., Scutellaria prostrata Jacq. ex Benth. In: Kunwar RW, Sher H, Bussmann RW. Ethnobotany of the Himalayas. Springer, Cham. doi: 10.1007/978-3-030-57408-6_222, pp. 1829-1836. 
Dangol DR, Jan HA, Abbasi AM, Kunwar RM, Bussmann RW, Paniagua-Zambrana NY. 2021. Chenopodium album L. In: Kunwar RW, Sher H, Bussmann RW. Ethnobotany of the Himalayas. Springer, Cham. doi: 10.1007/978-3-03057408-6_54, pp. 515-526.

Devkota A, Kunwar RM, Bussmann RW, Paniagua-Zambrana NY. 2021. Drymaria cordata (L.) Willd. ex Schult. In: Kunwar RW, Sher H, Bussmann RW. Ethnobotany of the Himalayas. Springer, Cham. doi: 10.1007/978-3-030-574086_86, pp. 795-804.

Dhamala MK, Khadka D, Kunwar RM, Bussmann RW, Paniagua-Zambrana NY. 2021. Cyathea spinulosa Wall. ex Hook. In: Kunwar RW, Sher H, Bussmann RW. Ethnobotany of the Himalayas. Springer, Cham. doi: 10.1007/978-3030-57408-6_72, pp. 669-678.

Dhruba Bijaya GC, Bhandari J, Gautam D, Kunwar RM, Jan HA, Abbasi AM, Bussmann RW, Paniagua-Zambrana NY. 2021. Juglans regia L. In: Kunwar RW, Sher H, Bussmann RW. Ethnobotany of the Himalayas. Springer, Cham. doi: 10.1007/978-3-030-57408-6_132, pp. 1123-1140.

Echeverría J, Paniagua-Zambrana NY, Bussmann RW, Romero C. 2020. Senecio adenophyllus Meyen \& Walp., Senecio atacamensis Phil., Senecio breviscapus DC., Senecio candollei Wedd., Senecio canescens (Bonpl.) Cuatrec, Senecio chinogeton Wedd., Senecio ctenophyllus Phil., Senecio filaginoides DC., Senecio haenkeanus Cuatrec., Senecio nutans Sch. Bip., Senecio olivaceobracteatus Ricardi \& Martic., Senecio pappii Ricardi \& Martic., Senecio proteus J. Remy var. proteus, Senecio pseudotites Grieseb., Senecio puchii Phil., Senecio scorzonerifolius Meyen \& Walp., Senecio smithioides Cabrera, Senecio teprosioides Turcz., Senecio volckmannii Phil., Senecio xerophilus Phil. var. xerophilus, Senecio zoellneri Martic. \& Quezada. In: Paniagua-Zambrana NY, Bussmann RW (Eds.) Ethnobotany of Mountain Regions - Ethnobotany of the Andes. Springer International Publishing, Cham. doi: 10.1007/978-3-31977093-2_265-1

Echeverría, J, Paniagua-Zambrana NY, Bussmann RW. 2020. Acaena magellanica (Lam.) Vahl. In: PaniaguaZambrana NY, Bussmann RW (Eds.) Ethnobotany of Mountain Regions - Ethnobotany of the Andes. Springer International Publishing, Cham. doi: 10.1007/978-3-319-77093-2_5-1

Echeverría, J, Paniagua-Zambrana NY, Bussmann RW. 2020. Adesmia atacamensis Phil., Adesmia erinacea Phil., Adesmia minor (Hook. \& Arn.) Burkart var. caespitosa (Phil.) Ulibarri \& Burkart, Adesmia rahmeri Phil., Adesmia spinosissima Meyen, Adesmia subterranea Clos. In: Paniagua-Zambrana NY, Bussmann RW (Eds.) Ethnobotany of Mountain Regions - Ethnobotany of the Andes. Springer International Publishing, Cham. doi: 10.1007/978-3-31977093-2_9-1

Echeverría, J, Paniagua-Zambrana NY, Bussmann RW. 2020. Airampoa ayrampo (Azara) Doweld. In: PaniaguaZambrana NY, Bussmann RW (Eds.) Ethnobotany of Mountain Regions - Ethnobotany of the Andes. Springer International Publishing, Cham. doi: 10.1007/978-3-319-77093-2_13-1

Echeverría, J, Paniagua-Zambrana NY, Bussmann RW. 2020. Allionia incarnata L. In: Paniagua-Zambrana NY, Bussmann RW (Eds.) Ethnobotany of Mountain Regions - Ethnobotany of the Andes. Springer International Publishing, Cham. doi: 10.1007/978-3-319-77093-2_14-1

Echeverría, J, Paniagua-Zambrana NY, Bussmann RW. 2020. Anthemis arvensis L. In: Paniagua-Zambrana NY, Bussmann RW (Eds.) Ethnobotany of Mountain Regions - Ethnobotany of the Andes. Springer International Publishing, Cham. doi: 10.1007/978-3-319-77093-2_24-1

Echeverría, J, Paniagua-Zambrana NY, Bussmann RW. 2020. Argyrochosma nivea (Poir.) Windham. In: PaniaguaZambrana NY, Bussmann RW (Eds.) Ethnobotany of Mountain Regions - Ethnobotany of the Andes. Springer International Publishing, Cham. doi: 10.1007/978-3-319-77093-2_27-1

Echeverría, J, Paniagua-Zambrana NY, Bussmann RW. 2020. Astragalus arequipensis Vogel. In: PaniaguaZambrana NY, Bussmann RW (Eds.) Ethnobotany of Mountain Regions - Ethnobotany of the Andes. Springer International Publishing, Cham. doi: 10.1007/978-3-319-77093-2_29-1

Echeverría, J, Paniagua-Zambrana NY, Bussmann RW. 2020. Atriplex glaucescens Phil., Atriplex imbricata (Moq.) D. Dietr. var. imbricata, Atriplex madariagae Phil. In: Paniagua-Zambrana NY, Bussmann RW (Eds.) Ethnobotany of Mountain Regions - Ethnobotany of the Andes. Springer International Publishing, Cham. doi: 10.1007/978-3-31977093-2_30-1 
Echeverría, J, Paniagua-Zambrana NY, Bussmann RW. 2020. Azorella atacamensis G.M. Plunkett \& A.N. Nicolas, Azorella compacta Phil. In: Paniagua-Zambrana NY, Bussmann RW (Eds.) Ethnobotany of Mountain Regions Ethnobotany of the Andes. Springer International Publishing, Cham. doi: 10.1007/978-3-319-77093-2_32-1

Echeverría, J, Paniagua-Zambrana NY, Bussmann RW. 2020. Bellardia trixago (L.) All. In: Paniagua-Zambrana NY, Bussmann RW (Eds.) Ethnobotany of Mountain Regions - Ethnobotany of the Andes. Springer International Publishing, Cham. doi: 10.1007/978-3-319-77093-2_36-1

Echeverría, J, Paniagua-Zambrana NY, Bussmann RW. 2020. Browningia candelaris (Meyen) Britton \& Rose. In: Paniagua-Zambrana NY, Bussmann RW (Eds.) Ethnobotany of Mountain Regions - Ethnobotany of the Andes. Springer International Publishing, Cham. doi: 10.1007/978-3-319-77093-2_43-1

Echeverría, J, Paniagua-Zambrana NY, Bussmann RW. 2020. Bryantiella glutinosa (Phil.) J.M. Porter. In: PaniaguaZambrana NY, Bussmann RW (Eds.) Ethnobotany of Mountain Regions - Ethnobotany of the Andes. Springer International Publishing, Cham. doi: 10.1007/978-3-319-77093-2_45-1

Echeverría, J, Paniagua-Zambrana NY, Bussmann RW. 2020. Caiophora chuquitensis (Meyen) Urb. \& Gilg, Caiophora rosulata (Wedd.) Urb. \& Gilg. In: Paniagua-Zambrana NY, Bussmann RW (Eds.) Ethnobotany of Mountain Regions - Ethnobotany of the Andes. Springer International Publishing, Cham. doi: 10.1007/978-3-319-77093-2_49-2

Echeverría, J, Paniagua-Zambrana NY, Bussmann RW. 2020. Carpobrotus chilensis (Molina) N.E. Br. In: PaniaguaZambrana NY, Bussmann RW (Eds.) Ethnobotany of Mountain Regions - Ethnobotany of the Andes. Springer International Publishing, Cham. doi: 10.1007/978-3-319-77093-2_55-1

Echeverría, J, Paniagua-Zambrana NY, Bussmann RW. 2020. Chara sp. In: Paniagua-Zambrana NY, Bussmann RW (Eds.) Ethnobotany of Mountain Regions - Ethnobotany of the Andes. Springer International Publishing, Cham. doi: 10.1007/978-3-319-77093-2_65-1

Echeverría, J, Paniagua-Zambrana NY, Bussmann RW. 2020. Cheilanthes pruinata Kaulf. In: Paniagua-Zambrana NY, Bussmann RW (Eds.) Ethnobotany of Mountain Regions - Ethnobotany of the Andes. Springer International Publishing, Cham. doi: 10.1007/978-3-319-77093-2_66-1

Echeverría, J, Paniagua-Zambrana NY, Bussmann RW. 2020. Chersodoma arequipensis (Cuatrec.) Cuatrec., Chersodoma jodopappa (Sch. Bip.) Cabrera. In: Paniagua-Zambrana NY, Bussmann RW (Eds.) Ethnobotany of Mountain Regions - Ethnobotany of the Andes. Springer International Publishing, Cham. doi: 10.1007/978-3-31977093-2_68-2

Echeverría, J, Paniagua-Zambrana NY, Bussmann RW. 2020. Chorizanthe commissuralis J. Remy. In: PaniaguaZambrana NY, Bussmann RW (Eds.) Ethnobotany of Mountain Regions - Ethnobotany of the Andes. Springer International Publishing, Cham. doi: 10.1007/978-3-319-77093-2_469-1

Echeverría, J, Paniagua-Zambrana NY, Bussmann RW. 2020. Cistanthe amarantoides (Phil.) Carolin ex Hershkovitz, Cistanthe celosioides (Phil.) Carolin ex Hershkovitz. In: Paniagua-Zambrana NY, Bussmann RW (Eds.) Ethnobotany of Mountain Regions - Ethnobotany of the Andes. Springer International Publishing, Cham. doi: 10.1007/978-3-31977093-2_73-1

Echeverría, J, Paniagua-Zambrana NY, Bussmann RW. 2020. Corryocactus brevistylus (K. Schum. ex Vaupel) Britton \& Rose. In: Paniagua-Zambrana NY, Bussmann RW (Eds.) Ethnobotany of Mountain Regions - Ethnobotany of the Andes. Springer International Publishing, Cham. doi: 10.1007/978-3-319-77093-2_80-1

Echeverría, J, Paniagua-Zambrana NY, Bussmann RW. 2020. Cortaderia speciosa (Nees \& Meyen) Stapf. In: Paniagua-Zambrana NY, Bussmann RW (Eds.) Ethnobotany of Mountain Regions - Ethnobotany of the Andes. Springer International Publishing, Cham. doi: 10.1007/978-3-319-77093-2_81-2

Echeverría, J, Paniagua-Zambrana NY, Bussmann RW. 2020. Cryptantha hispida (Phil.) Reiche. In: PaniaguaZambrana NY, Bussmann RW (Eds.) Ethnobotany of Mountain Regions - Ethnobotany of the Andes. Springer International Publishing, Cham. doi: 10.1007/978-3-319-77093-2_85-1

Echeverría, J, Paniagua-Zambrana NY, Bussmann RW. 2020. Cumulopuntia sphaerica (C.F. Först.) E.F. Anderson. In: Paniagua-Zambrana NY, Bussmann RW (Eds.) Ethnobotany of Mountain Regions - Ethnobotany of the Andes. Springer International Publishing, Cham. doi: 10.1007/978-3-319-77093-2_89-2 
Echeverría, J, Paniagua-Zambrana NY, Bussmann RW. 2020. Diplostephium cinereum Cuatrec. In: PaniaguaZambrana NY, Bussmann RW (Eds.) Ethnobotany of Mountain Regions - Ethnobotany of the Andes. Springer International Publishing, Cham. doi: 10.1007/978-3-319-77093-2_100-2

Echeverría, J, Paniagua-Zambrana NY, Bussmann RW. 2020. Distichlis spicata (L.) Greene. In: Paniagua-Zambrana NY, Bussmann RW (Eds.) Ethnobotany of Mountain Regions - Ethnobotany of the Andes. Springer International Publishing, Cham. doi: 10.1007/978-3-319-77093-2_102-1

Echeverría, J, Paniagua-Zambrana NY, Bussmann RW. 2020. Dunalia spinosa (Meyen) Dammer. In: PaniaguaZambrana NY, Bussmann RW (Eds.) Ethnobotany of Mountain Regions - Ethnobotany of the Andes. Springer International Publishing, Cham. doi: 10.1007/978-3-319-77093-2_105-2

Echeverría, J, Paniagua-Zambrana NY, Bussmann RW. 2020. Errazurizia multifoliolata (Clos) I.M. Johnst. In: Paniagua-Zambrana NY, Bussmann RW (Eds.) Ethnobotany of Mountain Regions - Ethnobotany of the Andes. Springer International Publishing, Cham. doi: 10.1007/978-3-319-77093-2_111-1

Echeverría, J, Paniagua-Zambrana NY, Bussmann RW. 2020. Erythranthe glabrata (Kunth) G.L. Nesom. In: PaniaguaZambrana NY, Bussmann RW (Eds.) Ethnobotany of Mountain Regions - Ethnobotany of the Andes. Springer International Publishing, Cham. doi: 10.1007/978-3-319-77093-2_117-1

Echeverría, J, Paniagua-Zambrana NY, Bussmann RW. 2020. Euphorbia klotzschii Oudejans var. klotzschii. In: Paniagua-Zambrana NY, Bussmann RW (Eds.) Ethnobotany of Mountain Regions - Ethnobotany of the Andes. Springer International Publishing, Cham. doi: 10.1007/978-3-319-77093-2_119-1

Echeverría, J, Paniagua-Zambrana NY, Bussmann RW. 2020. Fabiana bryoides Phil., Fabiana densa J. Remy, Fabiana denudata Miers, Fabiana ramulosa (Wedd.) Hunz. \& Barboza, Fabiana squamata Phil. In: Paniagua-Zambrana NY, Bussmann RW (Eds.) Ethnobotany of Mountain Regions - Ethnobotany of the Andes. Springer International Publishing, Cham. doi: 10.1007/978-3-319-77093-2_120-1

Echeverría, J, Paniagua-Zambrana NY, Bussmann RW. 2020. Festuca chrysophylla Phil. In: Paniagua-Zambrana NY, Bussmann RW (Eds.) Ethnobotany of Mountain Regions - Ethnobotany of the Andes. Springer International Publishing, Cham. doi: 10.1007/978-3-319-77093-2_121-2

Echeverría, J, Paniagua-Zambrana NY, Bussmann RW. 2020. Geoffroea decorticans (Gillies ex Hook. \& Arn.) Burkart. In: Paniagua-Zambrana NY, Bussmann RW (Eds.) Ethnobotany of Mountain Regions - Ethnobotany of the Andes. Springer International Publishing, Cham. doi: 10.1007/978-3-319-77093-2_128-2

Echeverría, J, Paniagua-Zambrana NY, Bussmann RW. 2020. Gilia laciniata Ruiz \& Pav. In: Paniagua-Zambrana NY, Bussmann RW (Eds.) Ethnobotany of Mountain Regions - Ethnobotany of the Andes. Springer International Publishing, Cham. doi: 10.1007/978-3-319-77093-2_130-1

Echeverría, J, Paniagua-Zambrana NY, Bussmann RW. 2020. Grindelia tarapacana Phil. In: Paniagua-Zambrana NY, Bussmann RW (Eds.) Ethnobotany of Mountain Regions - Ethnobotany of the Andes. Springer International Publishing, Cham. doi: 10.1007/978-3-319-77093-2_133-1

Echeverría, J, Paniagua-Zambrana NY, Bussmann RW. 2020. Haagocereus fascicularis (Meyen) F. Ritter. In: Paniagua-Zambrana NY, Bussmann RW (Eds.) Ethnobotany of Mountain Regions - Ethnobotany of the Andes. Springer International Publishing, Cham. doi: 10.1007/978-3-319-77093-2_135-1

Echeverría, J, Paniagua-Zambrana NY, Bussmann RW. 2020. Halerpestes cymbalaria (Pursh) Greene. In: PaniaguaZambrana NY, Bussmann RW (Eds.) Ethnobotany of Mountain Regions - Ethnobotany of the Andes. Springer International Publishing, Cham. doi: 10.1007/978-3-319-77093-2_136-1

Echeverría, J, Paniagua-Zambrana NY, Bussmann RW. 2020. Haplopappus rigidus Phil. In: Paniagua-Zambrana NY, Bussmann RW (Eds.) Ethnobotany of Mountain Regions - Ethnobotany of the Andes. Springer International Publishing, Cham. doi: 10.1007/978-3-319-77093-2_137-2

Echeverría, J, Paniagua-Zambrana NY, Bussmann RW. 2020. Hoffmannseggia doellii Phil. ssp. doellii, Hoffmannseggia eremophila (Phil.) Burkart ex Ulibarri. In: Paniagua-Zambrana NY, Bussmann RW (Eds.) Ethnobotany of Mountain Regions - Ethnobotany of the Andes. Springer International Publishing, Cham. doi: 10.1007/978-3-319-77093-2_140-2 
Echeverría, J, Paniagua-Zambrana NY, Bussmann RW. 2020. Hypochaeris chondrilloides (A. Gray) Cabrera, Hypochaeris taraxacoides Ball. In: Paniagua-Zambrana NY, Bussmann RW (Eds.) Ethnobotany of Mountain Regions - Ethnobotany of the Andes. Springer International Publishing, Cham. doi: 10.1007/978-3-319-77093-2_145-1

Echeverría, J, Paniagua-Zambrana NY, Bussmann RW. 2020. Jaborosa caulescens Gillies \& Hook. In: PaniaguaZambrana NY, Bussmann RW (Eds.) Ethnobotany of Mountain Regions - Ethnobotany of the Andes. Springer International Publishing, Cham. doi: 10.1007/978-3-319-77093-2_151-2

Echeverría, J, Paniagua-Zambrana NY, Bussmann RW. 2020. Jarava leptostachya (Griseb.) F. Rojas. In: PaniaguaZambrana NY, Bussmann RW (Eds.) Ethnobotany of Mountain Regions - Ethnobotany of the Andes. Springer International Publishing, Cham. doi: 10.1007/978-3-319-77093-2_153-1

Echeverría, J, Paniagua-Zambrana NY, Bussmann RW. 2020. Junellia digitata (Phil.) Moldenke var. digitata, Junellia minima (Meyen) Moldenke, Junellia seriphioides (Gillies \& Hook. ex Hook.) Moldenke. In: Paniagua-Zambrana NY, Bussmann RW (Eds.) Ethnobotany of Mountain Regions - Ethnobotany of the Andes. Springer International Publishing, Cham. doi: 10.1007/978-3-319-77093-2_156-2

Echeverría, J, Paniagua-Zambrana NY, Bussmann RW. 2020. Lilaeopsis macloviana (Gand.) A.W. Hill. In: PaniaguaZambrana NY, Bussmann RW (Eds.) Ethnobotany of Mountain Regions - Ethnobotany of the Andes. Springer International Publishing, Cham. doi: 10.1007/978-3-319-77093-2_168-2

Echeverría, J, Paniagua-Zambrana NY, Bussmann RW. 2020. Limosella australis R. Br. In: Paniagua-Zambrana NY, Bussmann RW (Eds.) Ethnobotany of Mountain Regions - Ethnobotany of the Andes. Springer International Publishing, Cham. doi: 10.1007/978-3-319-77093-2_170-1

Echeverría, J, Paniagua-Zambrana NY, Bussmann RW. 2020. Lobivia formosa (Pfeiff.) Dodds var. formosa. In: Paniagua-Zambrana NY, Bussmann RW (Eds.) Ethnobotany of Mountain Regions - Ethnobotany of the Andes. Springer International Publishing, Cham. doi: 10.1007/978-3-319-77093-2_172-1

Echeverría, J, Paniagua-Zambrana NY, Bussmann RW. 2020. Lophopappus tarapacanus (Phil.) Cabrera. In: Paniagua-Zambrana NY, Bussmann RW (Eds.) Ethnobotany of Mountain Regions - Ethnobotany of the Andes. Springer International Publishing, Cham. doi: 10.1007/978-3-319-77093-2_174-1

Echeverría, J, Paniagua-Zambrana NY, Bussmann RW. 2020. Maihueniopsis boliviana (Salm-Dyck) R. Kiesling ssp. ignescens (Vaupel) Faúndez \& R. Kiesling, Maihueniopsis camachoi (Espinosa) F. Ritter. In: Paniagua-Zambrana NY, Bussmann RW (Eds.) Ethnobotany of Mountain Regions - Ethnobotany of the Andes. Springer International Publishing, Cham. doi: 10.1007/978-3-319-77093-2_178-2

Echeverría, J, Paniagua-Zambrana NY, Bussmann RW. 2020. Micromeria gilliesii Benth. In: Paniagua-Zambrana NY, Bussmann RW (Eds.) Ethnobotany of Mountain Regions - Ethnobotany of the Andes. Springer International Publishing, Cham. doi: 10.1007/978-3-319-77093-2_190-1

Echeverría, J, Paniagua-Zambrana NY, Bussmann RW. 2020. Moschopsis monocephala (Phil.) Reiche. In: PaniaguaZambrana NY, Bussmann RW (Eds.) Ethnobotany of Mountain Regions - Ethnobotany of the Andes. Springer International Publishing, Cham. doi: 10.1007/978-3-319-77093-2_194-2

Echeverría, J, Paniagua-Zambrana NY, Bussmann RW. 2020. Myriophyllum aquaticum (Vell.) Verdc. In: PaniaguaZambrana NY, Bussmann RW (Eds.) Ethnobotany of Mountain Regions - Ethnobotany of the Andes. Springer International Publishing, Cham. doi: 10.1007/978-3-319-77093-2_198-2

Echeverría, J, Paniagua-Zambrana NY, Bussmann RW. 2020. Myrosmodes nervosa (Kraenzl.) Novoa, C. Vargas \& Cisternas. In: Paniagua-Zambrana NY, Bussmann RW (Eds.) Ethnobotany of Mountain Regions - Ethnobotany of the Andes. Springer International Publishing, Cham. doi: 10.1007/978-3-319-77093-2_200-1

Echeverría, J, Paniagua-Zambrana NY, Bussmann RW. 2020. Neuontobotrys tarapacana (Phil.) Al-Shehbaz. In: Paniagua-Zambrana NY, Bussmann RW (Eds.) Ethnobotany of Mountain Regions - Ethnobotany of the Andes. Springer International Publishing, Cham. doi: 10.1007/978-3-319-77093-2_203-1

Echeverría, J, Paniagua-Zambrana NY, Bussmann RW. 2020. Nostocsp. In: Paniagua-Zambrana NY, Bussmann RW (Eds.) Ethnobotany of Mountain Regions - Ethnobotany of the Andes. Springer International Publishing, Cham. doi: 10.1007/978-3-319-77093-2_206-1 
Echeverría, J, Paniagua-Zambrana NY, Bussmann RW. 2020. Nototriche clandestina (Phil.) A.W. Hill, Nototriche estipulata A.W. Hill ex B.L. Burtt, Nototriche parviflora (Phil.) A.W. Hill. In: Paniagua-Zambrana NY, Bussmann RW (Eds.) Ethnobotany of Mountain Regions - Ethnobotany of the Andes. Springer International Publishing, Cham. doi: 10.1007/978-3-319-77093-2_207-1

Echeverría, J, Paniagua-Zambrana NY, Bussmann RW. 2020. Ombrophytum subterraneum (Aspl.) B. Hansen. In: Paniagua-Zambrana NY, Bussmann RW (Eds.) Ethnobotany of Mountain Regions - Ethnobotany of the Andes. Springer International Publishing, Cham. doi: 10.1007/978-3-319-77093-2_209-1

Echeverría, J, Paniagua-Zambrana NY, Bussmann RW. 2020. Oreocereus leucotrichus (Phil.) Wagenkn. ex F. Ritter. In: Paniagua-Zambrana NY, Bussmann RW (Eds.) Ethnobotany of Mountain Regions - Ethnobotany of the Andes. Springer International Publishing, Cham. doi: 10.1007/978-3-319-77093-2_211-1

Echeverría, J, Paniagua-Zambrana NY, Bussmann RW. 2020. Oriastrum revolutum (Phil.) A.M.R. Davies, Oriastrum sphaeroidale Reiche. In: Paniagua-Zambrana NY, Bussmann RW (Eds.) Ethnobotany of Mountain Regions Ethnobotany of the Andes. Springer International Publishing, Cham. doi: 10.1007/978-3-319-77093-2_212-1

Echeverría, J, Paniagua-Zambrana NY, Bussmann RW. 2020. Oscillatoria tenuis C.Agardh ex Gomont. In: PaniaguaZambrana NY, Bussmann RW (Eds.) Ethnobotany of Mountain Regions - Ethnobotany of the Andes. Springer International Publishing, Cham. doi: 10.1007/978-3-319-77093-2_314-1

Echeverría, J, Paniagua-Zambrana NY, Bussmann RW. 2020. Oxychloe andina Phil. In: Paniagua-Zambrana NY, Bussmann RW (Eds.) Ethnobotany of Mountain Regions - Ethnobotany of the Andes. Springer International Publishing, Cham. doi: 10.1007/978-3-319-77093-2_217-1

Echeverría, J, Paniagua-Zambrana NY, Bussmann RW. 2020. Parastrephia lucida (Meyen) Cabrera, Parastrephia quadrangularis (Meyen) Cabrera, Parastrephia teretiuscula (Kuntze) Cabrera. In: Paniagua-Zambrana NY, Bussmann RW (Eds.) Ethnobotany of Mountain Regions - Ethnobotany of the Andes. Springer International Publishing, Cham. doi: 10.1007/978-3-319-77093-2_219-1

Echeverría, J, Paniagua-Zambrana NY, Bussmann RW. 2020. Paronychia microphylla Phil. In: Paniagua-Zambrana NY, Bussmann RW (Eds.) Ethnobotany of Mountain Regions - Ethnobotany of the Andes. Springer International Publishing, Cham. doi: 10.1007/978-3-319-77093-2_220-1

Echeverría, J, Paniagua-Zambrana NY, Bussmann RW. 2020. Pitraea cuneato-ovata (Cav.) Caro. In: PaniaguaZambrana NY, Bussmann RW (Eds.) Ethnobotany of Mountain Regions - Ethnobotany of the Andes. Springer International Publishing, Cham. doi: 10.1007/978-3-319-77093-2_235-1

Echeverría, J, Paniagua-Zambrana NY, Bussmann RW. 2020. Pseudognaphalium dysodes (Spreng.) S. E. Freire, Bayón \& C. Monti, Pseudognaphalium psilophyllum (Meyen \& Walp.) Anderb. In: Paniagua-Zambrana NY, Bussmann RW (Eds.) Ethnobotany of Mountain Regions - Ethnobotany of the Andes. Springer International Publishing, Cham. doi: 10.1007/978-3-319-77093-2_244-1

Echeverría, J, Paniagua-Zambrana NY, Bussmann RW. 2020. Pycnophyllum bryoides (Phil.) Rohrb., Pycnophyllum macropetalum Mattf. In: Paniagua-Zambrana NY, Bussmann RW (Eds.) Ethnobotany of Mountain Regions Ethnobotany of the Andes. Springer International Publishing, Cham. doi: 10.1007/978-3-319-77093-2_246-1

Echeverría, J, Paniagua-Zambrana NY, Bussmann RW. 2020. Reyesia juniperoides (Werderm.) D'Arcy. In: PaniaguaZambrana NY, Bussmann RW (Eds.) Ethnobotany of Mountain Regions - Ethnobotany of the Andes. Springer International Publishing, Cham. doi: 10.1007/978-3-319-77093-2_249-1

Echeverría, J, Paniagua-Zambrana NY, Bussmann RW. 2020. Stellaria chilensis Pedersen. In: Paniagua-Zambrana NY, Bussmann RW (Eds.) Ethnobotany of Mountain Regions - Ethnobotany of the Andes. Springer International Publishing, Cham.

Echeverría, J, Paniagua-Zambrana NY, Bussmann RW. 2020. Tarasa tarapacana (Phil.) Krapov., Tarasa tenella (Cav.) Krapov. In: Paniagua-Zambrana NY, Bussmann RW (Eds.) Ethnobotany of Mountain Regions - Ethnobotany of the Andes. Springer International Publishing, Cham. doi: 10.1007/978-3-319-77093-2_279-1

Echeverría, J, Paniagua-Zambrana NY, Bussmann RW. 2020. Thelypteris argentina (Hieron.) Abbiatti, Thelypteris sp. In: Paniagua-Zambrana NY, Bussmann RW (Eds.) Ethnobotany of Mountain Regions - Ethnobotany of the Andes. Springer International Publishing, Cham. doi: 10.1007/978-3-319-77093-2_283-1 
Echeverría, J, Paniagua-Zambrana NY, Bussmann RW. 2020. Tiquilia atacamensis (Phil.) A.T. Richardson, Tiquilia paronychoides A.T. Richardson. In: Paniagua-Zambrana NY, Bussmann RW (Eds.) Ethnobotany of Mountain Regions - Ethnobotany of the Andes. Springer International Publishing, Cham. doi: 10.1007/978-3-319-77093-2_285-1

Echeverría, J, Paniagua-Zambrana NY, Bussmann RW. 2020. Tribulus terrestris L. In: Paniagua-Zambrana NY, Bussmann RW (Eds.) Ethnobotany of Mountain Regions - Ethnobotany of the Andes. Springer International Publishing, Cham. doi: 10.1007/978-3-319-77093-2_287-1

Echeverría, J, Paniagua-Zambrana NY, Bussmann RW. 2020. Trichocereus atacamensis (Phil.) Backeb. In: PaniaguaZambrana NY, Bussmann RW (Eds.) Ethnobotany of Mountain Regions - Ethnobotany of the Andes. Springer International Publishing, Cham. doi: 10.1007/978-3-319-77093-2_288-1

Echeverría, J, Paniagua-Zambrana NY, Bussmann RW. 2020. Trichocline caulescens Phil. In: Paniagua-Zambrana NY, Bussmann RW (Eds.) Ethnobotany of Mountain Regions - Ethnobotany of the Andes. Springer International Publishing, Cham.

Echeverría, J, Paniagua-Zambrana NY, Bussmann RW. 2020. Trixis cacalioides (Kunth) D. Don. In: PaniaguaZambrana NY, Bussmann RW (Eds.) Ethnobotany of Mountain Regions - Ethnobotany of the Andes. Springer International Publishing, Cham. doi: 10.1007/978-3-319-77093-2_291-1

Echeverría, J, Paniagua-Zambrana NY, Bussmann RW. 2020. Xanthium spinosum L. In: Paniagua-Zambrana NY, Bussmann RW (Eds.) Ethnobotany of Mountain Regions - Ethnobotany of the Andes. Springer International Publishing, Cham. doi: 10.1007/978-3-319-77093-2_300-1

Fairbarin J, Preston D, Paniagua-Zambrana NY, Mass G, Yevara M, Beck S. 2001. Pastoreo y cambios ambientales en el altiplano de Tarija, Bolivia. En Historia, Ambiente y Sociedad en Tarija, Bolivia. Beck, S, N. Paniagua \& D. Preston (eds.). Instituto de Ecología, UMSA/Escuela de Geografía/Universidad de Leeds. Ed. Grafica Latina. La Paz, Bolivia.

Fayvush G, Aleksanyan A, Batsatsashvili K, Kikvidze Z, Khutsishvili M, Maisaia I, Sikharulidze S, Tchelidze D, PaniaguaZambrana NY, Bussmann RW. 2017. Helichrysum rubicundum (K. Koch) Bornm. In: Bussmann RW (ed.) Ethnobotany of the Caucasus, Springer International Publishing, Cham. doi: 10.1007/978-3-319-49412-8_77

Fayvush G, Aleksanyan A, Batsatsashvili K, Kikvidze Z, Khutsishvili M, Maisaia I, Sikharulidze S, Tchelidze D, PaniaguaZambrana NY, Bussmann RW. 2017. Polygonatum glaberrimum C. Koch., Polygonatum orientale Desf. In: Bussmann RW (ed.) Ethnobotany of the Caucasus, Springer International Publishing, Cham. doi: 10.1007/978-3-319-49412-8_79

Fayvush G, Aleksanyan A, Batsatsashvili K, Mehdiyeva N, Kikvidze Z, Khutsishvili M, Maisaia I, Sikharulidze S, Tchelidze D, Alizade V, Paniagua-Zambrana NY, Bussmann RW. 2017. Physalis alkekengi L. In: Bussmann RW (ed.) Ethnobotany of the Caucasus, Springer International Publishing, Cham. doi: 10.1007/978-3-319-49412-8_81

Fayvush G, Aleksanyan A, Bussmann RW. 2017. Ethnobotany of the Caucasus - Armenia. In: Bussmann RW (ed.) Ethnobotany of the Caucasus, Springer International Publishing, Cham. doi: 10.1007/978-3-319-49412-8_18

Fayvush G, Aleksanyan A, Mehdiyeva N, Alizade V, Batsatsashvili K, Kikvidze Z, Khutsishvili M, Maisaia I, Sikharulidze S, Tchelidze D, Paniagua-Zambrana NY, Bussmann RW. 2017. Allium paradoxum (M. Bieb.) G. Don, Allium ursinum L., Allium victorialis L. In: Bussmann RW (ed.) Ethnobotany of the Caucasus, Springer International Publishing, Cham. doi: 10.1007/978-3-319-49412-8_135

Fayvush G, Aleksanyan A, Mehdiyeva N, Alizade V, Batsatsashvili K, Kikvidze Z, Khutsishvili M, Maisaia I, Sikharulidze S, Tchelidze D, Paniagua-Zambrana NY, Bussmann RW. 2017. Asparagus officinalis L., Asparagus verticillatus L. In: Bussmann RW (ed.) Ethnobotany of the Caucasus, Springer International Publishing, Cham. doi: 10.1007/978-3-31949412-8_100

Fayvush G, Aleksanyan A, Mehdiyeva N, Alizade V, Paniagua-Zambrana NY, Bussmann RW. 2017. Hippophae rhamnoides L. In: Bussmann RW (ed.) Ethnobotany of the Caucasus, Springer International Publishing, Cham. doi: 10.1007/978-3-319-49412-8_90

Fayvush G, Aleksanyan A, Mehdiyeva N, Alizade V, Paniagua-Zambrana NY, Bussmann RW. 2017. Ornithogalum ponticum Zahar. In: Bussmann RW (ed.) Ethnobotany of the Caucasus, Springer International Publishing, Cham. doi: 10.1007/978-3-319-49412-8_146 
Fayvush G, Aleksanyan A, Mehdiyeva N, Alizade V, Paniagua-Zambrana NY, Bussmann RW. 2017. Peganum harmala L. In: Bussmann RW (ed.) Ethnobotany of the Caucasus, Springer International Publishing, Cham. doi: 10.1007/978-3-319-49412-8_134

Foster R, Paniagua-Zambrana NY, Alverson B, Rojas J. 2000. Rapid Color Guide \# 54: Plantas del Tahuamanu. Pando Bolivia. Field Museum of Natural History of Chicago. Chicago, IL.

Foster R, Rojas, J, Paniagua-Zambrana NY, Alverson, B, Torrico G. 2000. Flora y Vegetación. En Bolivia: Pando, Río Tahuamanu. Rapid Biological Inventories 01. Alverson et al. (eds). Field Museum of Natural History of Chicago. Chicago, IL.

Gauli K, Kumar Sharma S, Kunwar RM, Bussmann RW, Paniagua-Zambrana NY. 2021. Cymbopogon citratus (DC.) Stapf In: Kunwar RW, Sher H, Bussmann RW. Ethnobotany of the Himalayas. Springer, Cham. doi: 10.1007/978-3030-57408-6_73, pp. 679-686.

Gautam S, Kunwar L, Dhami N, Kunwar RM, Bussmann RW, Paniagua-Zambrana NY. 2021. Murraya koenigii (L.) Spreng. In: Kunwar RW, Sher H, Bussmann RW. Ethnobotany of the Himalayas. Springer, Cham. doi: 10.1007/978-3030-57408-6_155, pp. 1317-1326.

Ghimire N, Kunwar RM, Hussain W, Abbasi AM, Bussmann RW, Paniagua-Zambrana NY. 2021. Betula utilis D. Don In: Kunwar RW, Sher H, Bussmann RW. Ethnobotany of the Himalayas. Springer, Cham. doi: 10.1007/978-3-03057408-6_37, pp. 369-380.

Ghorbani A, Esmaeili S, Bussmann RW, Batsatsashvili K, Kikvidze Z, Paniagua-Zambrana NY, Khutsishvili M, Maisaia I, Sikharulidze S, Tchelidze D. 2020. n: Batsatsashvili K, Kikvidze Z, Bussmann RW (Eds.) Ethnobotany of Mountain Regions Far Eastern Europe. Springer International Publishing, Cham.

Ghorbani A, Nasab Farzaneh K, Bussmann RW, Batsatsashvili K, Kikvidze Z, Paniagua-Zambrana NY, Khutsishvili M, Maisaia I, Sikharulidze S, Tchelidze, D 2019. Melilotus officinalis (L.) Lam. In: Batsatsashvili K, Kikvidze Z, Bussmann RW (Eds.) Ethnobotany of Mountain Regions Far Eastern Europe. Springer International Publishing, Cham. doi: 10.1007/978-3-319-77088-8_90-2

Goutam KR, Kunwar RM, Bussmann RW, Paniagua-Zambrana NY. 2021. Myrica esculenta Buch.-Ham. ex D. Don. In: Kunwar RW, Sher H, Bussmann RW. Ethnobotany of the Himalayas. Springer, Cham. doi: 10.1007/978-3-030-574086_157, pp. 1337-1344.

Gubhaju MR, Jan HA, Kunwar RM, Sher H, Ur Rahman I, Hussain W, Abbasi AM, Bussmann RW, Paniagua-Zambrana NY. 2021. Bergenia ciliata Sternb. In: Kunwar RW, Sher H, Bussmann RW. Ethnobotany of the Himalayas. Springer, Cham. doi: 10.1007/978-3-030-57408-6_36, pp. 353-368.

Gurung A, Kunwar RM, Bussmann RW, Paniagua-Zambrana NY. 2021. Ocimum gratissimum Lam. In: Kunwar RW, Sher H, Bussmann RW. Ethnobotany of the Himalayas. Springer, Cham. doi: 10.1007/978-3-030-57408-6_163, pp. 1369-1378.

Gyawali R, Kunwar RM, Bussmann RW, Paniagua-Zambrana NY. 2021. Citrus medica L. In: Kunwar RW, Sher H, Bussmann RW. Ethnobotany of the Himalayas. Springer, Cham. doi: 10.1007/978-3-030-57408-6_59, pp. 567-582.

Gyawali S, Luintel S, Kunwar RM, Bussmann RW, Paniagua-Zambrana NY. 2021. Thalictrum cultratum Wall., Thalictrum foetidum L., Thalictrum foliolosum DC. In: Kunwar RW, Sher H, Bussmann RW. Ethnobotany of the Himalayas. Springer, Cham. doi: 10.1007/978-3-030-57408-6_245, pp. 2015-2026.

Gyawali S, Luintel S, Kunwar RM, Jan HA, Bussmann RW, Paniagua-Zambrana NY. 2021. Jasminum humile L. In: Kunwar RW, Sher H, Bussmann RW. Ethnobotany of the Himalayas. Springer, Cham. doi: 10.1007/978-3-030-574086_130, pp. 1103-1110.

Gyawali S, Luintel S, Luintel H, Kunwar RM, Bussmann RW. 2021. Sapium insigne (Royle) Benth. \& Hook. f. In: Kunwar RW, Sher H, Bussmann RW. Ethnobotany of the Himalayas. Springer, Cham. doi: 10.1007/978-3-030-57408-6_215, pp. 1785-1790.

Gyawali S, Luintel S, Thapa S, Kunwar RM, Bussmann RW, Paniagua-Zambrana NY. 2021. Anisomeles indica (L.) Kuntze In: Kunwar RW, Sher H, Bussmann RW. Ethnobotany of the Himalayas. Springer, Cham. doi: 10.1007/978-3030-57408-6_24, pp. 249-252. 
Hussain W, Bussmann RW, Paniagua-Zambrana NY. 2021. Acantholimon lycopodioides (Girard) Boiss. In: Kunwar RW, Sher H, Bussmann RW. Ethnobotany of the Himalayas. Springer, Cham. doi: 10.1007/978-3-030-57408-6_7, pp. 119-122.

Hussain W, Bussmann RW, Paniagua-Zambrana NY. 2021. Gentiana kurroo Roye In: Kunwar RW, Sher H, Bussmann RW. Ethnobotany of the Himalayas. Springer, Cham. doi: 10.1007/978-3-030-57408-6_107, pp. 971-976.

Hussain W, Bussmann RW, Paniagua-Zambrana NY. 2021. Lepidium apetalum Willd., Lepidium sativum L. In: Kunwar RW, Sher H, Bussmann RW. Ethnobotany of the Himalayas. Springer, Cham. doi: 10.1007/978-3-030-574086_141, pp. 1189-1196.

Hussain W, Bussmann RW, Paniagua-Zambrana NY. 2021. Onosma hispida Wall. ex G. Don In: Kunwar RW, Sher H, Bussmann RW. Ethnobotany of the Himalayas. Springer, Cham. doi: 10.1007/978-3-030-57408-6_165, pp. 1389-1392.

Hussain W, Jan HA, Abbasi AM, Kunwar RM, Bussmann RW, Paniagua-Zambrana NY. 2021. Ephedra gerardiana Wall. ex Stapf In: Kunwar RW, Sher H, Bussmann RW. Ethnobotany of the Himalayas. Springer, Cham. doi: 10.1007/978-3-030-57408-6_92, pp. 867-874.

Hussain W, Jan HA, Bussmann RW, Paniagua-Zambrana NY. 2021. Withania coagulans (Stocks) Dunal, Withania somnifera (L.) Dunal In: Kunwar RW, Sher H, Bussmann RW. Ethnobotany of the Himalayas. Springer, Cham. doi: 10.1007/978-3-030-57408-6_259, pp. 2139-2146.

Hussain W, Sher H, Kunwar RM, Bussmann RW, Paniagua-Zambrana NY. 2021. Nepeta brachyantha Rech.f. \& Edelb., Nepeta cataria L., Nepeta connata Royle ex Benth., Nepeta leucolaena Benth. ex Hook.f., Nepeta hindostana (Roth.) Hainus., Nepeta podostachys Benth. In: Kunwar RW, Sher H, Bussmann RW. Ethnobotany of the Himalayas. Springer, Cham. doi: 10.1007/978-3-030-57408-6_161, pp. 1353-1358.

Jan HA, (Bussmann RW, Paniagua-Zambrana NY. 2021. Achillea millefolium L. ssp. millefolium In: Kunwar RW, Sher H, Bussmann RW. Ethnobotany of the Himalayas. Springer, Cham. doi: 10.1007/978-3-030-57408-6_8, pp. $123-132$.

Jan HA, Abbasi AM, Ali M, Bussmann RW, Paniagua-Zambrana NY. 2021. Urtica dioica L. In: Kunwar RW, Sher H, Bussmann RW. Ethnobotany of the Himalayas. Springer, Cham. doi: 10.1007/978-3-030-57408-6_252, pp. 2067-2078.

Jan HA, Abbasi AM, Bussmann RW, Paniagua-Zambrana NY 2021. Datura stramonium L. In: Kunwar RW, Sher $\mathrm{H}$, Bussmann RW. Ethnobotany of the Himalayas. Springer, Cham. doi: 10.1007/978-3-030-57408-6_77, pp. 725-734.

Jan HA, Abbasi AM, Bussmann RW, Paniagua-Zambrana NY. 2021. Cannabis sativa L. In: Kunwar RW, Sher H, Bussmann RW. Ethnobotany of the Himalayas. Springer, Cham. doi: 10.1007/978-3-030-57408-6_45, pp. 443-450.

Jan HA, Abbasi AM, Bussmann RW, Paniagua-Zambrana NY. 2021. Duchesnea indica (Andews) Teschem. In: Kunwar RW, Sher H, Bussmann RW. Ethnobotany of the Himalayas. Springer, Cham. doi: 10.1007/978-3-030-57408-6_88, pp. 821-824.

Jan HA, Abbasi AM, Bussmann RW, Paniagua-Zambrana NY. 2021. Olea ferruginea Royle In: Kunwar RW, Sher H, Bussmann RW. Ethnobotany of the Himalayas. Springer, Cham. doi: 10.1007/978-3-030-57408-6_2164 pp. 13791388.

Jan HA, Abbasi AM, Hussain W, Bussmann RW, Paniagua-Zambrana NY. 2021. Indigofera heterantha (Wall. ex Brandis) var. gerardiana (Wall.ex Baker) Ali In: Kunwar RW, Sher H, Bussmann RW. Ethnobotany of the Himalayas. Springer, Cham. doi: 10.1007/978-3-030-57408-6_124, pp. 1085-1090.

Jan HA, Abbasi AM, Kunwar RM, Bussmann RW, Paniagua-Zambrana NY Sher H, Ur Rahman I. 2021. Capparis spinosa L. In: Kunwar RW, Sher H, Bussmann RW. Ethnobotany of the Himalayas. Springer, Cham. doi: 10.1007/9783-030-57408-6_46, pp. 451-460

Jan HA, Abbasi AM, Kunwar RM, Bussmann RW, Paniagua-Zambrana NY. 2021. Papaver dubium L., Papaver nudicaule L., Papaver somniferum L. In: Kunwar RW, Sher H, Bussmann RW. Ethnobotany of the Himalayas. Springer, Cham. doi: 10.1007/978-3-030-57408-6_172, pp. 1431-1438.

Jan HA, Abbasi AM, Sher H, Bussmann RW, Paniagua-Zambrana NY. 2021. Peganum harmala L In: Kunwar RW, Sher H, Bussmann RW. Ethnobotany of the Himalayas. Springer, Cham. doi: 10.1007/978-3-030-57408-6_177, pp. 14611470. 
Jan HA, Ali M, Kunwar RM, Bussmann RW, Paniagua-Zambrana NY. 2021. Malva neglecta Wallr. In: Kunwar RW, Sher H, Bussmann RW. Ethnobotany of the Himalayas. Springer, Cham. doi: 10.1007/978-3-030-57408-6_148, pp. $1239-1246$.

Jan HA, Bussmann RW, Paniagua-Zambrana NY, Sher, H. 2021. Ethnobotany of Mountain Regions Himalaya - The Hindukush and Karakoram. In: Kunwar RW, Sher H, Bussmann RW. Ethnobotany of the Himalayas. Springer, Cham. doi: 10.1007/978-3-030-57408-6_2, pp. 3-34.

Jan HA, Bussmann RW, Paniagua-Zambrana NY. 2021. Allium carolinianum DC., Allium griffithianum Boiss., Allium humile Kunth, Allium jacquemointii Regel, Allium oreoprasum Schrenk In: Kunwar RW, Sher H, Bussmann RW. Ethnobotany of the Himalayas. Springer, Cham. doi: 10.1007/978-3-030-57408-6_16, pp. 183-202.

Jan HA, Bussmann RW, Paniagua-Zambrana NY. 2021. Atropa acuminata Royle In: Kunwar RW, Sher H, Bussmann RW. Ethnobotany of the Himalayas. Springer, Cham. doi: 10.1007/978-3-030-57408-6_33, pp. 323-326.

Jan HA, Bussmann RW, Paniagua-Zambrana NY. 2021. Epilobium angustifolium L., Epilobium latifolium L. ssp. latifolium L., Epilobium leiophyllum Hausskn., Epilobium royleanum Hausskn. In: Kunwar RW, Sher H, Bussmann RW. Ethnobotany of the Himalayas. Springer, Cham. doi: 10.1007/978-3-030-57408-6_93, pp. 875-878.

Jan HA, Bussmann RW, Paniagua-Zambrana NY. 2021. Lamium album L. In: Kunwar RW, Sher H, Bussmann RW. Ethnobotany of the Himalayas. Springer, Cham. doi: 10.1007/978-3-030-57408-6_138, pp. 1173-1178.

Jan HA, Bussmann RW, Paniagua-Zambrana NY. 2021. Launaea procumbens (Roxb.) Ramayya \& Rajagopal In: Kunwar RW, Sher H, Bussmann RW. Ethnobotany of the Himalayas. Springer, Cham. doi: 10.1007/978-3-030-574086_139, pp. 1179-1182.

Jan HA, Bussmann RW, Paniagua-Zambrana NY. 2021. Tribulus terrestris L. In: Kunwar RW, Sher H, Bussmann RW. Ethnobotany of the Himalayas. Springer, Cham. doi: 10.1007/978-3-030-57408-6_248, pp. 2045-2052.

Jan HA, Hussain W, Abbasi AM, Bussmann RW, Paniagua-Zambrana NY. 2021. Cichorium intybus L. In: Kunwar RW, Sher H, Bussmann RW. Ethnobotany of the Himalayas. Springer, Cham. doi: 10.1007/978-3-030-57408-6_56, pp. 541546.

Jan HA, Hussain W, Abbasi AM, Kunwar RM, Bussmann RW, Paniagua-Zambrana NY. 2021. Geranium collinum Steph. ex Willd., Geranium nepalense Sweet, Geranium wallichianum D. Don ex Sweet In: Kunwar RW, Sher H, Bussmann RW. Ethnobotany of the Himalayas. Springer, Cham. doi: 10.1007/978-3-030-57408-6_110, pp. 985-994.

Jan HA, Hussain W, Abbasi AM, Sher H, Bussmann RW, Paniagua-Zambrana NY. 2021. Elaeagnus angustifolia L. var. angustifolia L. In: Kunwar RW, Sher H, Bussmann RW. Ethnobotany of the Himalayas. Springer, Cham. doi: 10.1007/978-3-030-57408-6_90, pp. 855-862.

Jan HA, Hussain W, Bussmann RW, Paniagua-Zambrana NY. 2021. Arnebia euchroma (Royle) I.M. Johnst. In: Kunwar RW, Sher H, Bussmann RW. Ethnobotany of the Himalayas. Springer, Cham. doi: 10.1007/978-3-030-57408-6_27, pp. 267-270.

Jan HA, Hussain W, Bussmann RW, Paniagua-Zambrana NY. 2021. Codonopsis clematidea (Schrenk ex Fisch. \& C.A. Mey.) C.B. Clarke In: Kunwar RW, Sher H, Bussmann RW. Ethnobotany of the Himalayas. Springer, Cham. doi: 10.1007/978-3-030-57408-6_61, pp. 591-594.

Jan HA, Hussain W, Bussmann RW, Paniagua-Zambrana NY. 2021. Fumaria indica Pugsley In: Kunwar RW, Sher H, Bussmann RW. Ethnobotany of the Himalayas. Springer, Cham. doi: 10.1007/978-3-030-57408-6_106, pp. 965-970.

Jan HA, Hussain W, Kunwar RM, Bussmann RW, Paniagua-Zambrana NY. 2021. Medicago sativa L. In: Kunwar RW, Sher H, Bussmann RW. Ethnobotany of the Himalayas. Springer, Cham. doi: 10.1007/978-3-030-57408-6_150, pp. 1257-1264.

Jan HA, Sher H, Bussmann RW, Paniagua-Zambrana NY, Ur Rahman, I (2021) Buxus wallichiana Baill. In: Kunwar RW, Sher H, Bussmann RW. Ethnobotany of the Himalayas. Springer, Cham. doi: 10.1007/978-3-030-57408-6_42, pp. 427-430.

Jan HA, Sher H, Hussain W, Ur Rahman I, Kunwar RM, Bussmann RW, Paniagua-Zambrana NY. 2021. Mentha arvensis L., Mentha longifolia (L.) L., Mentha royleana Benth. In: Kunwar RW, Sher H, Bussmann RW. Ethnobotany of the Himalayas. Springer, Cham. doi: 10.1007/978-3-030-57408-6_151, pp. 1265-1278. 
Jan HA, Sher H, Hussain W, Ur-Rahman, I, Bussmann RW, Paniagua-Zambrana NY. 2021. Artemisia absinthium L., Artemisia biennis Willd., Artemisia bigelovii A. Gray, Artemisia brevifolia Wall. ex DC, Artemisia scoparia Waldst. ex Kit., Artemisia vulgaris L. In: Kunwar RW, Sher H, Bussmann RW. Ethnobotany of the Himalayas. Springer, Cham. doi: 10.1007/978-3-030-57408-6_28, pp. 271-288.

Jan HA, Sher H, Kunwar RM, Bussmann RW, Paniagua-Zambrana NY. 2021. Incarvillea emodi Chatterjee In: Kunwar RW, Sher H, Bussmann RW. Ethnobotany of the Himalayas. Springer, Cham. doi: 10.1007/978-3-030-57408-6_123, pp. 1081-1084.

Jan HA, Sher H, Ur Rahman I, Abbasi AM, Bussmann RW, Paniagua-Zambrana NY. 2021. Primula denticulata Smith, Primula macrophylla D. Don, Primula reptans Hook. ex Watt In: Kunwar RW, Sher H, Bussmann RW. Ethnobotany of the Himalayas. Springer, Cham. doi: 10.1007/978-3-030-57408-6_196, pp. 1589-1596.

Jan HA, Sher H, Ur Rahman I, Bussmann RW, Paniagua-Zambrana NY. 2021. Celastrus paniculatus Willd. In: Kunwar RW, Sher H, Bussmann RW. Ethnobotany of the Himalayas. Springer, Cham. doi: 10.1007/978-3-030-57408-6_52, pp. 505-508.

Jan HA, Sher H, Ur Rahman I, Hussain W, Abbasi AM, Bussmann RW, Paniagua-Zambrana NY. 2021. Pinus gerardiana Wall. ex Lamb., Pinus roxburghii Sarg., Pinus wallichiana A.B. Jacks. In: Kunwar RW, Sher H, Bussmann RW. Ethnobotany of the Himalayas. Springer, Cham. doi: 10.1007/978-3-030-57408-6_186, pp. 1519-1530.

Jan HA, Sher H, Ur Rahman I, Hussain W, Kunwar RM, Bussmann RW, Paniagua-Zambrana NY. 2021. Hypericum perforatum L. In: Kunwar RW, Sher H, Bussmann RW. Ethnobotany of the Himalayas. Springer, Cham. doi: 10.1007/978-3-030-57408-6_122, pp. 1065-1074.

Jan HA, Sher H, Ur Rahman I, Hussain W, Kunwar RM, Bussmann RW, Paniagua-Zambrana NY. 2021. Rheum emodi Wall., Rheum tibeticum Maxim. ex Hook. f. In: Kunwar RW, Sher H, Bussmann RW. Ethnobotany of the Himalayas. Springer, Cham. doi: 10.1007/978-3-030-57408-6_203, pp. 1665-1672.

Joshi LR, Bhatta S, Paudel HR, Kunwar RM, Jan HA, Abbasi AM, Bussmann RW, Paniagua-Zambrana NY. 2021. Justicia adathoda L. In: Kunwar RW, Sher H, Bussmann RW. Ethnobotany of the Himalayas. Springer, Cham. doi: 10.1007/978-3-030-57408-6_136, pp. 1163-1172.

Joshi N, Kunwar RM, Jan HA, Bussmann RW, Paniagua-Zambrana NY. 2021. Quercus baloot Griff., Quercus dilatata Lindl, Quercus incana W. Bartram, Quercus lanata Sm., In: Kunwar RW, Sher H, Bussmann RW. Ethnobotany of the Himalayas. Springer, Cham. doi: 10.1007/978-3-030-57408-6_201, pp. 1633-1646.

Joshi N, Sher H, Ur Rahman I, Bussmann RW, Paniagua-Zambrana NY. 2021. Dioscorea bulbifera L., Dioscorea deltoidea Wall. ex Kunth, In: Kunwar RW, Sher H, Bussmann RW. Ethnobotany of the Himalayas. Springer, Cham. doi: 10.1007/978-3-030-57408-6_83, pp. 769-778.

Joshi Shrestha S, Kunwar RM, Bussmann RW, Paniagua-Zambrana NY. 2021. Senecio nudicaulis Buch.-Ham. ex C.B. Clarke In: Kunwar RW, Sher H, Bussmann RW. Ethnobotany of the Himalayas. Springer, Cham. doi: 10.1007/978-3030-57408-6_225, pp. 1845-1854.

Kafle MR, Kunwar RM, Jan HA, Abbasi AM, Bussmann RW, Paniagua-Zambrana NY. 2021. Woodfordia fruticosa (L.) Kurz In: Kunwar RW, Sher H, Bussmann RW. Ethnobotany of the Himalayas. Springer, Cham. doi: 10.1007/978-3-03057408-6_260, pp. 2147-2158.

Karki G, Kunwar RM, Acharya, RP, Bussmann RW, Paniagua-Zambrana NY. 2021. Paris polyphylla Sm. In: Kunwar RW, Sher H, Bussmann RW. Ethnobotany of the Himalayas. Springer, Cham. doi: 10.1007/978-3-030-57408-6_174, pp. 1441-1448.

Kattel AM, Sher H, Ur Rahman I, Hussain W, Jan HA, Abbasi AM, Kunwar RM, Bussmann RW, Paniagua-Zambrana NY. 2021. Plantago depressa Willd, Plantago lanceolata L., Plantago major L., Plantago ovata Fersk. In: Kunwar RW, Sher H, Bussmann RW. Ethnobotany of the Himalayas. Springer, Cham. doi: 10.1007/978-3-030-57408-6_188, pp. 1539-1554.

Khanal M, Lekhak HD, Kunwar RM, Bussmann RW, Paniagua-Zambrana NY. 2021. Cucumis sativus L., Cucumis sativus var. hardwickii (Royle) Alef In: Kunwar RW, Sher H, Bussmann RW. Ethnobotany of the Himalayas. Springer, Cham. doi: 10.1007/978-3-030-57408-6_68, pp. 635-642. 
Khaniya L, Bhattarai R, Kunwar RM, Jan HA, Hussain W, Abbasi AM, Bussmann RW, Paniagua-Zambrana NY. 2021. Rubus ellipticus Sm., Rubus foliolosus Weihe \& Nees, Rubus fruticosus L., Rubus irritans Focke In: Kunwar RW, Sher H, Bussmann RW. Ethnobotany of the Himalayas. Springer, Cham. doi: 10.1007/978-3-030-57408-6_208, pp. 17171734.

Khaniya L, Kunwar RM, Bussmann RW, Paniagua-Zambrana NY. 2021. Bidens biternata (Lour.) Merr. \& Sherff In: Kunwar RW, Sher H, Bussmann RW. Ethnobotany of the Himalayas. Springer, Cham. doi: 10.1007/978-3-030-574086_38, pp. 381-390.

Khaniya L, Kunwar RM, Bussmann RW, Paniagua-Zambrana NY. 2021. Leea indica (Burm. f.) Merr. In: Kunwar RW, Sher H, Bussmann RW. Ethnobotany of the Himalayas. Springer, Cham. doi: 10.1007/978-3-030-57408-6_140, pp. 1183-1188.

Khatri Chhetri NB, Kunwar RM, Jan HA, Bussmann RW., Paniagua-Zambrana NY, Ali, M. 2021. Ziziphus mauritiana Lam, Ziziphus jujuba Mill In: Kunwar RW, Sher H, Bussmann RW. Ethnobotany of the Himalayas. Springer, Cham. doi: 10.1007/978-3-030-57408-6_262, pp. 2173-2185.

Khatri S, Jan HA, Abbasi AM, Kunwar RM, Bussmann RW, Paniagua-Zambrana NY. 2021. Cassia fistula L., Cassia occidentalis L. In: Kunwar RW, Sher H, Bussmann RW. Ethnobotany of the Himalayas. Springer, Cham. doi: 10.1007/978-3-030-57408-6_49, pp. 477-490

Kunwar L, Gautam S, Dhami N, Jan HA, Ali M, Hussain W, Abbasi AM, Sher H, Kunwar RM, Bussmann RW, PaniaguaZambrana NY. 2021. Solanum aculeatissimum Jacq., Solanum nigrum L. var. nigrum, Solanum surattense Burm. $\mathrm{f}$. In: Kunwar RW, Sher H, Bussmann RW. Ethnobotany of the Himalayas. Springer, Cham. doi: 10.1007/978-3-03057408-6_230, pp. 1881-1906.

Kunwar RM, Bussmann RW, Paniagua-Zambrana NY. 2021. Andrographis paniculata (Burm. f.) Wall. In: Kunwar RW, Sher H, Bussmann RW. Ethnobotany of the Himalayas. Springer, Cham. doi: 10.1007/978-3-030-57408-6_22, pp. 235238.

Kunwar RM, Bussmann RW, Paniagua-Zambrana NY. 2021. Aquilaria sinensis (Loureiro) Sprengel, Aquilaria yunnanensis S.C. Huang. In: Kunwar RW, Sher H, Bussmann RW. Ethnobotany of the Himalayas. Springer, Cham. doi: 10.1007/978-3-030-57408-6_25, pp. 253-256.

Kunwar RM, Bussmann RW, Paniagua-Zambrana NY. 2021. Holboellia latifolia Wall. In: Kunwar RW, Sher H, Bussmann RW. Ethnobotany of the Himalayas. Springer, Cham. doi: 10.1007/978-3-030-57408-6_119, pp. 1049-1054.

Kunwar RM, Bussmann RW, Paniagua-Zambrana NY. 2021. Hyoscyamus nigerL. In: Kunwar RW, Sher H, Bussmann RW. Ethnobotany of the Himalayas. Springer, Cham. doi: 10.1007/978-3-030-57408-6_121, pp. 1059-1064.

Kunwar RM, Bussmann RW, Paniagua-Zambrana NY. 2021. Nardostachys grandiflora DC. In: Kunwar RW, Sher H, Bussmann RW. Ethnobotany of the Himalayas. Springer, Cham. doi: 10.1007/978-3-030-57408-6_159, pp. 1345-1348.

Kunwar RM, Bussmann RW, Paniagua-Zambrana NY. 2021. Neopicrorhiza schrophularïflora (Pennell) D.Y. Hong In: Kunwar RW, Sher H, Bussmann RW. Ethnobotany of the Himalayas. Springer, Cham. doi: 10.1007/978-3-030-574086_160, pp. 1349-1352.

Kunwar, RA, Bussmann RW, Paniagua-Zambrana NY. 2021. Ajuga brachystemon Maxim., Ajuga parviflora Benth. in Wall. In: Kunwar RW, Sher H, Bussmann RW. Ethnobotany of the Himalayas. Springer, Cham. doi: 10.1007/978-3030-57408-6_15, pp. 177-182.

Lamichhane G, Poudel P, Poudel HR, Thapa R, Kunwar RM, Bussmann RW, Paniagua-Zambrana NY. 2021. Cirsium verutum (D. Don) Spreng. In: Kunwar RW, Sher H, Bussmann RW. Ethnobotany of the Himalayas. Springer, Cham. doi: 10.1007/978-3-030-57408-6_58, pp. 555-566.

Luintel S, Gyawali S, Kunwar RTM, Abbasi AM, Bussmann RW, Paniagua-Zambrana NY. 2021. Taraxacum campylodes G.E. Haglund, Taraxacum officinale F.H. Wigg., Taraxacum sikkimense Hand.-Mazz. In: Kunwar RW, Sher H, Bussmann RW. Ethnobotany of the Himalayas. Springer, Cham. doi: 10.1007/978-3-030-57408-6_422, pp. 19771990.

Macía MJ, Cámara Leret R, Paniagua-Zambrana NY. 2014. Uso de palmas por poblaciones locales. In Balslev $\mathrm{H}_{\text {, }}$ Macía MJ, Navarrete H (Eds.). Cosecha de palmas en el noroeste de Sudamérica - las bases científicas para su manejo y conservación. Pontificia Universidad Católica de Ecuador, Quito. Pp. 57-86. 
Mahat Kunwar L, Mahat R, Thapa A, Mahat R, Kunwar RM, Bussmann RW. 2021. Hydrocotyle nepalensis Hook. In: Kunwar RW, Sher H, Bussmann RW. Ethnobotany of the Himalayas. Springer, Cham. doi: 10.1007/978-3-030-574086_120, pp. 1055-1058.

Mahat R, Thapa A, Bhattarai R, Kunwar RM, Bussmann RW, Paniagua-Zambrana NY. 2021. Hedychium spicatum Buch.-Ham. ex Sm. In: Kunwar RW, Sher H, Bussmann RW. Ethnobotany of the Himalayas. Springer, Cham. doi: 10.1007/978-3-030-57408-6_114, pp. 1015-1020.

Mahat R, Thapa A, Kunwar RM, Jan HA, Hussain W, Bussmann RW, Paniagua-Zambrana NY. 2021. Cuscuta reflexa Roxb. In: Kunwar RW, Sher H, Bussmann RW. Ethnobotany of the Himalayas. Springer, Cham. doi: 10.1007/978-3030-57408-6_71, pp. 661-668.

Malla KJ, Kunwar RM, Jan HA, Bussmann RW, Paniagua-Zambrana NY. 2021. Boerhavia diffusa L. In: Kunwar RW, Sher H, Bussmann RW. Ethnobotany of the Himalayas. Springer, Cham. doi: 10.1007/978-3-030-57408-6_40, pp. 403408.

Mehdiyeva N, Alizade V, Batsatsashvili K, Kikvidze Z, Khutsishvili M, Maisaia I, Sikharulidze S, Tchelidze D, PaniaguaZambrana NY, Bussmann RW. 2017. Acer laetum C. A. Mey, Acer platanoides L., Acer pseudoplatanus L., Acer velutinum Boiss. In: Bussmann RW (ed.) Ethnobotany of the Caucasus, Springer International Publishing, Cham. doi: 10.1007/978-3-319-49412-8_24

Mehdiyeva N, Alizade V, Batsatsashvili K, Kikvidze Z, Khutsishvili M, Maisaia I, Sikharulidze S, Tchelidze D, PaniaguaZambrana NY, Bussmann RW. 2017. Agrimonia eupatoria L. In: Bussmann RW (ed.) Ethnobotany of the Caucasus, Springer International Publishing, Cham. doi: 10.1007/978-3-319-49412-8_32

Mehdiyeva N, Alizade V, Batsatsashvili K, Kikvidze Z, Khutsishvili M, Maisaia I, Sikharulidze S, Tchelidze D, PaniaguaZambrana NY, Bussmann RW. 2017. Asplenium trichomanes L. In: Bussmann RW (ed.) Ethnobotany of the Caucasus, Springer International Publishing, Cham. doi: 10.1007/978-3-319-49412-8_76

Mehdiyeva N, Alizade V, Batsatsashvili K, Kikvidze Z, Khutsishvili M, Maisaia I, Sikharulidze S, Tchelidze D, PaniaguaZambrana NY, Bussmann RW. 2017. Castanea sativa Mill. In: Bussmann RW (ed.) Ethnobotany of the Caucasus, Springer International Publishing, Cham. doi: 10.1007/978-3-319-49412-8_58

Mehdiyeva N, Alizade V, Batsatsashvili K, Kikvidze Z, Khutsishvili M, Maisaia I, Sikharulidze S, Tchelidze D, PaniaguaZambrana NY, Bussmann RW. 2017. Primula macrocalyx Bunge, Primula woronowii Losinsk. In: Bussmann RW (ed.) Ethnobotany of the Caucasus, Springer International Publishing, Cham. doi: 10.1007/978-3-319-49412-8_85

Mehdiyeva N, Alizade V, Batsatsashvili K, Kikvidze Z, Khutsishvili M, Maisaia I, Sikharulidze S, Tchelidze D, PaniaguaZambrana NY, Bussmann RW. 2017. Pyrethrum parthenifolium Willd., Pyrethrum roseum (Adams) M. Bieb. In: Bussmann RW (ed.) Ethnobotany of the Caucasus, Springer International Publishing, Cham. doi: 10.1007/978-3-31949412-8_28

Mehdiyeva N, Alizade V, Batsatsashvili K, Kikvidze Z, Khutsishvili M, Maisaia I, Sikharulidze S, Tchelidze D, PaniaguaZambrana NY, Bussmann RW. 2017. Viola arvensis L., Viola odorata L. In: Bussmann RW (ed.) Ethnobotany of the Caucasus, Springer International Publishing, Cham. doi: 10.1007/978-3-319-49412-8_109

Mehdiyeva N, Alizade V, Fayvush G, Aleksanyan A, Batsatsashvili K, Kikvidze Z, Khutsishvili M, Maisaia I, Sikharulidze S, Tchelidze D, Paniagua-Zambrana NY, Bussmann RW. 2017. Atriplex hortensis L., Atriplex tatarica L. In: Bussmann RW (ed.) Ethnobotany of the Caucasus, Springer International Publishing, Cham. doi: 10.1007/978-3-319-494128_126

Mehdiyeva N, Alizade V, Fayvush G, Aleksanyan A, Paniagua-Zambrana NY, Bussmann RW. 2017. Capparis sicula Vieill. subsp. herbacea (Willd.) Inocencio, D. Rivera, Obón \& Alcaraz. In: Bussmann RW (ed.) Ethnobotany of the Caucasus, Springer International Publishing, Cham. doi: 10.1007/978-3-319-49412-8_112

Mehdiyeva N, Alizade V, Paniagua-Zambrana NY, Bussmann RW. 2017. Acantholimon hohenackeri (Jaub. \& Spach) Boiss. In: Bussmann RW (ed.) Ethnobotany of the Caucasus, Springer International Publishing, Cham. doi: 10.1007/978-3-319-49412-8_96

Mehdiyeva N, Alizade V, Paniagua-Zambrana NY, Bussmann RW. 2017. Alcea hyrcana (Grossh.) Grossh., Alcea lenkoranica Iljin, Alcea rugosa Alef., Alcea tabrisiana (Boiss.et Buhse) Iljin. In: Bussmann RW (ed.) Ethnobotany of the Caucasus, Springer International Publishing, Cham. doi: 10.1007/978-3-319-49412-8_114 
Mehdiyeva N, Alizade V, Paniagua-Zambrana NY, Bussmann RW. 2017. Anacamptis pyramidalis (L.) Rich. In: Bussmann RW (ed.) Ethnobotany of the Caucasus, Springer International Publishing, Cham. doi: 10.1007/978-3-31949412-8_132

Mehdiyeva N, Alizade V, Paniagua-Zambrana NY, Bussmann RW. 2017. Clinopodium nepeta (L.) Kuntze. In: Bussmann RW (ed.) Ethnobotany of the Caucasus, Springer International Publishing, Cham. doi: 10.1007/978-3-31949412-8_40

Mehdiyeva N, Alizade V, Paniagua-Zambrana NY, Bussmann RW. 2017. Carthamus tinctorius L. In: Bussmann RW (ed.) Ethnobotany of the Caucasus, Springer International Publishing, Cham. doi: 10.1007/978-3-319-49412-8_1215

Mehdiyeva N, Alizade V, Paniagua-Zambrana NY, Bussmann RW. 2017. Consolida orientalis (J. Gay) Schrödinger. In: Bussmann RW (ed.) Ethnobotany of the Caucasus, Springer International Publishing, Cham. doi: 10.1007/978-3319-49412-8_94

Mehdiyeva N, Alizade V, Paniagua-Zambrana NY, Bussmann RW. 2017. Cotinus coggygria Scop. In: Bussmann RW (ed.) Ethnobotany of the Caucasus, Springer International Publishing, Cham. doi: 10.1007/978-3-319-49412-8_48

Mehdiyeva N, Alizade V, Paniagua-Zambrana NY, Bussmann RW. 2017. Crocus speciosus M. Bieb. In: Bussmann RW (ed.) Ethnobotany of the Caucasus, Springer International Publishing, Cham. doi: 10.1007/978-3-319-49412-8_68

Mehdiyeva N, Alizade V, Paniagua-Zambrana NY, Bussmann RW. 2017. Cyperus rotundus L. In: Bussmann RW (ed.) Ethnobotany of the Caucasus, Springer International Publishing, Cham. doi: 10.1007/978-3-319-49412-8_99

Mehdiyeva N, Alizade V, Paniagua-Zambrana NY, Bussmann RW. 2017. Datisca cannabina L. In: Bussmann RW (ed.) Ethnobotany of the Caucasus, Springer International Publishing, Cham. doi: 10.1007/978-3-319-49412-8_139

Mehdiyeva N, Alizade V, Paniagua-Zambrana NY, Bussmann RW. 2017. Ecballium elaterium (L.) A. Rich. In: Bussmann RW (ed.) Ethnobotany of the Caucasus, Springer International Publishing, Cham. doi: 10.1007/978-3-31949412-8_107

Mehdiyeva N, Alizade V, Paniagua-Zambrana NY, Bussmann RW. 2017. Euphorbia seguieriana Neck. In: Bussmann RW (ed.) Ethnobotany of the Caucasus, Springer International Publishing, Cham. doi: 10.1007/978-3-319-49412-8_30

Mehdiyeva N, Alizade V, Paniagua-Zambrana NY, Bussmann RW. 2017. Froriepia subpinnata (Ldb.) Bail. In: Bussmann RW (ed.) Ethnobotany of the Caucasus, Springer International Publishing, Cham. doi: 10.1007/978-3-31949412-8_49

Mehdiyeva N, Alizade V, Paniagua-Zambrana NY, Bussmann RW. 2017. Gleditsia caspica Desf. In: Bussmann RW (ed.) Ethnobotany of the Caucasus, Springer International Publishing, Cham. doi: 10.1007/978-3-319-49412-8_67

Mehdiyeva N, Alizade V, Paniagua-Zambrana NY, Bussmann RW. 2017. Iris pseudacorus L. In: Bussmann RW (ed.) Ethnobotany of the Caucasus, Springer International Publishing, Cham. doi: 10.1007/978-3-319-49412-8_145

Mehdiyeva N, Alizade V, Paniagua-Zambrana NY, Bussmann RW. 2017. Juncus acutus L., Juncus articulatus L In: Bussmann RW (ed.) Ethnobotany of the Caucasus, Springer International Publishing, Cham. doi: 10.1007/978-3-31949412-8_106

Mehdiyeva N, Alizade V, Paniagua-Zambrana NY, Bussmann RW. 2017. Lavatera thuringiaca L. In: Bussmann RW (ed.) Ethnobotany of the Caucasus, Springer International Publishing, Cham. doi: 10.1007/978-3-319-49412-8_66

Mehdiyeva N, Alizade V, Paniagua-Zambrana NY, Bussmann RW. 2017. Lilium ledebourii (Baker) Boiss. In: Bussmann RW (ed.) Ethnobotany of the Caucasus, Springer International Publishing, Cham. doi: 10.1007/978-3-31949412-8_92

Mehdiyeva N, Alizade V, Paniagua-Zambrana NY, Bussmann RW. 2017. Limonium meyeri (Boiss.) Kuntze. In: Bussmann RW (ed.) Ethnobotany of the Caucasus, Springer International Publishing, Cham. doi: 10.1007/978-3-31949412-8_111

Mehdiyeva N, Alizade V, Paniagua-Zambrana NY, Bussmann RW. 2017. Myosotis arvensis (L.) Hill. In: Bussmann RW (ed.) Ethnobotany of the Caucasus, Springer International Publishing, Cham. doi: 10.1007/978-3-319-49412-8_103

Mehdiyeva N, Alizade V, Paniagua-Zambrana NY, Bussmann RW. 2017. Nigella sativa L. In: Bussmann RW (ed.) Ethnobotany of the Caucasus, Springer International Publishing, Cham. doi: 10.1007/978-3-319-49412-8_31 
Mehdiyeva N, Alizade V, Paniagua-Zambrana NY, Bussmann RW. 2017. Orchis caspia Trautv., Orchis mascula (L.) L., Orchis palustris Jacq., Orchis picta Loisel., Orchis simia Lam. In: Bussmann RW (ed.) Ethnobotany of the Caucasus, Springer International Publishing, Cham. doi: 10.1007/978-3-319-49412-8_83

Mehdiyeva N, Alizade V, Paniagua-Zambrana NY, Bussmann RW. 2017. Paeonia tenuifolia L. In: Bussmann RW (ed.) Ethnobotany of the Caucasus, Springer International Publishing, Cham. doi: 10.1007/978-3-319-49412-8_54

Mehdiyeva N, Alizade V, Paniagua-Zambrana NY, Bussmann RW. 2017. Papaver orientale L., Papaver somniferum L. In: Bussmann RW (ed.) Ethnobotany of the Caucasus, Springer International Publishing, Cham. doi: 10.1007/9783-319-49412-8_143

Mehdiyeva N, Alizade V, Paniagua-Zambrana NY, Bussmann RW. 2017. Parrotia persica (DC.) C. A. Mey. In: Bussmann RW (ed.) Ethnobotany of the Caucasus, Springer International Publishing, Cham. doi: 10.1007/978-3-31949412-8_136

Mehdiyeva N, Alizade V, Paniagua-Zambrana NY, Bussmann RW. 2017. Pimpinella aromatica M. Bieb., Pimpinella peregrina L., Pimpinella saxifraga L. In: Bussmann RW (ed.) Ethnobotany of the Caucasus, Springer International Publishing, Cham. doi: 10.1007/978-3-319-49412-8_105

Mehdiyeva N, Alizade V, Paniagua-Zambrana NY, Bussmann RW. 2017. Pistacia mutica Fisch. \& C.A. Mey. In: Bussmann RW (ed.) Ethnobotany of the Caucasus, Springer International Publishing, Cham. doi: 10.1007/978-3-31949412-8_118

Mehdiyeva N, Alizade V, Paniagua-Zambrana NY, Bussmann RW. 2017. Pterocarya pterocarpa (Michx.) Kunth ex Iljins. In: Bussmann RW (ed.) Ethnobotany of the Caucasus, Springer International Publishing, Cham. doi: 10.1007/978-3-319-49412-8_142

Mehdiyeva N, Alizade V, Paniagua-Zambrana NY, Bussmann RW. 2017. Rhus coriaria L. In: Bussmann RW (ed.) Ethnobotany of the Caucasus, Springer International Publishing, Cham. doi: 10.1007/978-3-319-49412-8_26

Mehdiyeva N, Alizade V, Paniagua-Zambrana NY, Bussmann RW. 2017. Silybum marianum(L.) Gaertn. In: Bussmann RW (ed.) Ethnobotany of the Caucasus, Springer International Publishing, Cham. doi: 10.1007/978-3-319-494128_110

Mehdiyeva N, Alizade V, Paniagua-Zambrana NY, Bussmann RW. 2017. Trapa hyrcana Woronow. In: Bussmann RW (ed.) Ethnobotany of the Caucasus, Springer International Publishing, Cham. doi: 10.1007/978-3-319-49412-8_41

Mehdiyeva N, Alizade V, Paniagua-Zambrana NY, Bussmann RW. 2017. Vitis sylvestris C. C. Gmel. In: Bussmann RW (ed.) Ethnobotany of the Caucasus, Springer International Publishing, Cham. doi: 10.1007/978-3-319-49412-8_86

Mehdiyeva N, Alizade V, Paniagua-Zambrana NY, Bussmann RW. 2017. Xeranthemum inapertum (L.) Mill. In: Bussmann RW (ed.) Ethnobotany of the Caucasus, Springer International Publishing, Cham. doi: 10.1007/978-3-31949412-8_56

Mehdiyeva N, Alizade V, Paniagua-Zambrana NY, Bussmann RW. 2017. Zelkova carpinifolia (Pall.) K. Koch. In: Bussmann RW (ed.) Ethnobotany of the Caucasus, Springer International Publishing, Cham. doi: 10.1007/978-3-31949412-8_52

Mehdiyeva N, Alizade V, Paniagua-Zambrana NY, Bussmann RW. 2017. Zosima absinthifolia Link. In: Bussmann RW (ed.) Ethnobotany of the Caucasus, Springer International Publishing, Cham. doi: 10.1007/978-3-319-49412-8_95

Mehdiyeva N, Fayvush G, Aleksanyan A, Alizade V, Paniagua-Zambrana NY, Bussmann RW. 2017. Bilacunaria microcarpa (M. Bieb.) Pimenov \& V.N. Tikhom. In: Bussmann RW (ed.) Ethnobotany of the Caucasus, Springer International Publishing, Cham. doi: 10.1007/978-3-319-49412-8_102

Mehdiyeva N, Fayvush G, Aleksanyan A, Alizade V, Paniagua-Zambrana NY, Bussmann RW. 2017. Carum carvi L., Carum caucasicum Boiss. In: Bussmann RW (ed.) Ethnobotany of the Caucasus, Springer International Publishing, Cham. doi: 10.1007/978-3-319-49412-8_21

Mehdiyeva N, Fayvush G, Aleksanyan A, Alizade V, Paniagua-Zambrana NY, Bussmann RW. 2017. Eremurus spectabilis M. Bieb. In: Bussmann RW (ed.) Ethnobotany of the Caucasus, Springer International Publishing, Cham. doi: 10.1007/978-3-319-49412-8_23 
Mehdiyeva N, Fayvush G, Aleksanyan A, Alizade V, Paniagua-Zambrana NY, Bussmann RW. 2017. Potentilla erecta (L.) Raeusch, Potentilla reptans L. In: Bussmann RW (ed.) Ethnobotany of the Caucasus, Springer International Publishing, Cham. doi: 10.1007/978-3-319-49412-8_122

Mehdiyeva N, Fayvush G, Alizade V, Aleksanyan A, Batsatsashvili K, Kikvidze Z, Khutsishvili M, Maisaia I, Sikharulidze S, Tchelidze D, Paniagua-Zambrana NY, Bussmann RW. 2017. Cichorium intybus L. In: Bussmann RW (ed.) Ethnobotany of the Caucasus, Springer International Publishing, Cham. doi: 10.1007/978-3-319-49412-8_50

Mehdiyeva N, Fayvush G, Alizade V, Aleksanyan A, Batsatsashvili K, Kikvidze Z, Khutsishvili M, Maisaia I, Sikharulidze S, Tchelidze D, Paniagua Zambrana, N.Y Bussmann RW. 2017. Glycyrrhiza glabra L. In: Bussmann RW (ed.) Ethnobotany of the Caucasus, Springer International Publishing, Cham. doi: 10.1007/978-3-319-49412-8_34

Mehdiyeva N, Fayvush G, Alizade V, Aleksanyan A, Batsatsashvili K, Kikvidze Z, Khutsishvili M, Maisaia I, Sikharulidze S, Tchelidze D, Paniagua-Zambrana NY, Bussmann RW. 2017. Taxus baccata L. In: Bussmann RW (ed.) Ethnobotany of the Caucasus, Springer International Publishing, Cham. doi: 10.1007/978-3-319-49412-8_82

Mehdiyeva N., Alizade V., Batsatsashvili K, Kikvidze Z, Khutsishvili M, Maisaia I, Sikharulidze S, Tchelidze D, PaniaguaZambrana NY, Bussmann RW. 2017. Cyclamen elegans Boiss.et Buhse, Cyclamen vernum Sweet. In: Bussmann RW (ed.) Ethnobotany of the Caucasus, Springer International Publishing, Cham. doi: 10.1007/978-3-319-49412-8_124

Mehdiyeva N., Alizade V., Batsatsashvili K, Kikvidze Z, Khutsishvili M, Maisaia I, Sikharulidze S, Tchelidze D, Paniagua Zambrana, N.Y Bussmann RW. 2017. Danae racemosa (L.) Moench, Ruscus hyrcanus Woron. In: Bussmann RW (ed.) Ethnobotany of the Caucasus, Springer International Publishing, Cham. doi: 10.1007/978-3-319-49412-8_44

Mehdiyeva N., Alizade V., Batsatsashvili K, Kikvidze Z, Khutsishvili M, Maisaia I, Sikharulidze S, Tchelidze D, PaniaguaZambrana NY, Bussmann RW. 2017. Daphne mezereum L. In: Bussmann RW (ed.) Ethnobotany of the Caucasus, Springer International Publishing, Cham. doi: 10.1007/978-3-319-49412-8_104

Mehdiyeva N., Alizade V., Batsatsashvili K, Kikvidze Z, Khutsishvili M, Maisaia I, Sikharulidze S, Tchelidze D, Paniagua Zambrana, N.Y Bussmann RW. 2017. Diospyros lotus L. In: Bussmann RW (ed.) Ethnobotany of the Caucasus, Springer International Publishing, Cham. doi: 10.1007/978-3-319-49412-8_130

Mehdiyeva N., Alizade V., Batsatsashvili K, Kikvidze Z, Khutsishvili M, Maisaia I, Sikharulidze S, Tchelidze D, PaniaguaZambrana NY, Bussmann RW. 2017. Filipendula ulmaria (L.) Maxim. In: Bussmann RW (ed.) Ethnobotany of the Caucasus, Springer International Publishing, Cham. doi: 10.1007/978-3-319-49412-8_74

Moazzami Farida SH, Ghorbani A, Bussmann RW, Batsatsashvili K, Kikvidze Z, Paniagua-Zambrana NY, Khutsishvili M, Maisaia I, Sikharulidze S, Tchelidze D. 2020. Conium maculatum L. In: Batsatsashvili K, Kikvidze Z, Bussmann RW (Eds.) Ethnobotany of Mountain Regions Far Eastern Europe. Springer International Publishing, Cham. doi: 10.1007/978-3-319-77088-8_148-2

Moazzami Farida SH, Ghorbani A, Bussmann RW, Batsatsashvili K, Kikvidze Z, Paniagua-Zambrana NY, Khutsishvili M, Maisaia I, Sikharulidze S, Tchelidze D. 2020. Cordia myxa L. In: Batsatsashvili K, Kikvidze Z, Bussmann RW (Eds.) Ethnobotany of Mountain Regions Far Eastern Europe. Springer International Publishing, Cham. doi: 10.1007/978-3319-77088-8_42-1

Moazzami Farida SH, Ghorbani A, Bussmann RW, Batsatsashvili K, Kikvidze Z, Paniagua-Zambrana NY, Khutsishvili M, Maisaia I, Sikharulidze S, Tchelidze D. 2020. Echium amoenum Fisch. \& C.A. Mey., Echium maculatum L. In: Batsatsashvili K, Kikvidze Z, Bussmann RW (Eds.) Ethnobotany of Mountain Regions Far Eastern Europe. Springer International Publishing, Cham. doi: 10.1007/978-3-319-77088-8_54-2

Moazzami Farida SH, Ghorbani A, Bussmann RW, Batsatsashvili K, Kikvidze Z, Paniagua-Zambrana NY, Khutsishvili M, Maisaia I, Sikharulidze S, Tchelidze D. 2020. Heliotropium europaeum L. In: Batsatsashvili K, Kikvidze Z, Bussmann RW (Eds.) Ethnobotany of Mountain Regions Far Eastern Europe. Springer International Publishing, Cham. doi: 10.1007/978-3-319-77088-8_69-2

Moazzami Farida SH, Ghorbani A, Bussmann RW, Batsatsashvili K, Kikvidze Z, Paniagua-Zambrana NY, Khutsishvili M, Maisaia I, Sikharulidze S, Tchelidze, D 2019. Bunium persicum (Boiss.) B. Fedtsch. In: Batsatsashvili K, Kikvidze Z, Bussmann RW (Eds.) Ethnobotany of Mountain Regions Far Eastern Europe. Springer International Publishing, Cham. doi: 10.1007/978-3-319-77088-8_30-1

Moazzami Farida SH, Ghorbani A, Bussmann RW, Batsatsashvili K, Kikvidze Z, Paniagua-Zambrana NY, Khutsishvili M, Maisaia I, Sikharulidze S, Tchelidze, D 2019. Anchusa azurea Retz. In: Batsatsashvili K, Kikvidze Z, Bussmann RW 
(Eds.) Ethnobotany of Mountain Regions Far Eastern Europe. Springer International Publishing, Cham. doi: 10.1007/978-3-319-77088-8_9-2

Moha, PP, Kunwar RM, Bussmann RW, Paniagua-Zambrana NY. 2021. 2021. Amomum subulatum Roxb. In: Kunwar RW, Sher H, Bussmann RW. Ethnobotany of the Himalayas. Springer, Cham. doi: 10.1007/978-3-030-57408-6_9, pp. 213-216.

Moraes M, Paniagua-Zambrana NY. 2006. Biología y Ecología de la Jatata (Geonoma deversa). In Ergueta et al. (Eds.) Jatata: Un recurso valioso para los habitantes del bosque tropical. Proyecto TROPICOS. pp. 57-72.

Moraes-R M, Paniagua-Zambrana NY Velarde-V MJ. 2008. Bases biológicas para el manejo forestal de productos derivados de palmeras de Bolivia. In: Memorias IV Reunión Nacional sobre Investigación Forestal, PROMAB, Cobija. Pp. 172-175.

Moraes-R M, Paniagua-Zambrana NY. 2007. Tasas productivas del motacú (Attalea phalerata, Arecaceae) hacia el manejo sostenible en Bolivia. In: Feyen J, Aguirre LF, Moraes-R M (eds.) Memorias Congreso Internacional sobre Desarrollo, Medio Ambiente y Recursos Naturales: Sostenibilidad a Múltiples Niveles y Escalas, Universidad Mayor de San Simón, Cochabamba. Pp. 16-37.

Moraes, M, Paniagua-Zambrana NY, Cámara Leret R, Balslev B, Macía MJ. 2014. Palmas útiles de Bolivia, Colombia, Ecuador y Perú. En: H. Balslev, M.J. Macía y H. Navarrete, editores. Cosecha de palmas en el noroeste de Sudamérica - las bases científicas para su manejo y conservación. Pontificia Universidad Católica de Ecuador, Quito. pp. 87-102.

Oli BN, Charmakar C, Kunwar RM, Bussmann RW, Paniagua-Zambrana NY, Jan HA, Sher H, Ur Rahman I, Ali, M. 2021. Valeriana hardwickii Wall. var. hoffmeisteri (KI.) Clarke, Valeriana jatamansi Jones, Valeriana officinalis L. In: Kunwar RW, Sher H, Bussmann RW. Ethnobotany of the Himalayas. Springer, Cham. doi: 10.1007/978-3-030-574086_253, pp. 2079-2100.

Pandey J, Kunwar RM, Bussmann RW, Paniagua-Zambrana NY. 2021. Tinospora cordifolia (Willd.) Miers ex Hook. f. \& Thomson, J., Tinospora sinensis (Lour.) Merr. In: Kunwar RW, Sher H, Bussmann RW. Ethnobotany of the Himalayas. Springer, Cham. doi: 10.1007/978-3-030-57408-6_247, pp. 2037-2044.

Paneru YR, Chalise P, Sher H, Ur Rahman I, Kunwar RM, Bussmann RW, Paniagua-Zambrana NY. 2021. Dactylorhiza hatagirea (D. Don) Soo In: Kunwar RW, Sher H, Bussmann RW. Ethnobotany of the Himalayas. Springer, Cham. doi: 10.1007/978-3-030-57408-6_75, pp. 695-704.

Paneru YR, Sher H, Ur Rahman I, Hussain W, Abbasi AM, Bussmann RW, Paniagua-Zambrana NY. 2021. Podophyllum hexandrum Royle In: Kunwar RW, Sher H, Bussmann RW. Ethnobotany of the Himalayas. Springer, Cham. doi: 10.1007/978-3-030-57408-6_192, pp. 1561-1568.

Paniagua-Zambrana NY, Bussmann RW 2021. Casos se implementación del Protocolo de_Nagoya en Bolivia: la ethnobotánica de los Chácobo Pacahuara en el siglo XXI. In: Delgado Burgoa, JMF, Silvestre Rojas, CA (eds). Acances teórico metodológicos y exoeriencias de diálogo intercientífico en países en países Andino Amazónicos. Ministerio de Educación, Viceministerio de Ciencia y Tecnología, Dirección General de Ciencia y Tecnología. La Paz, Bolivia pp. 57-91.

Paniagua-Zambrana NY, Bussmann RW, Echeverría J, Romero C. 2020. Acmella oppositifolia (Lam.) R.K. Jansen. In: Paniagua-Zambrana NY, Bussmann RW (Eds.) Ethnobotany of Mountain Regions - Ethnobotany of the Andes. Springer International Publishing, Cham. doi: 10.1007/978-3-319-77093-2_8-1

Paniagua-Zambrana NY, Bussmann RW, Echeverría J, Romero C. 2020. Aloysia deserticola (Phil.) Lu-Irving \& O'Leary, Aloysia triphylla Royle. In: Paniagua-Zambrana NY, Bussmann RW (Eds.) Ethnobotany of Mountain Regions - Ethnobotany of the Andes. Springer International Publishing, Cham. doi: 10.1007/978-3-319-77093-2_182

Paniagua-Zambrana NY, Bussmann RW, Echeverría J, Romero C. 2020. Amaranthus caudatus L., Amaranthus deflexus L., Amaranthus hybridus L., Amaranthus retroflexus L., Amaranthus spinosus L. In: Paniagua-Zambrana NY, Bussmann RW (Eds.) Ethnobotany of Mountain Regions - Ethnobotany of the Andes. Springer International Publishing, Cham. doi: 10.1007/978-3-319-77093-2_20-1

Paniagua-Zambrana NY, Bussmann RW, Echeverría J, Romero C. 2020. Ambrosia arborescens Mill, Ambrosia artemisioides Meyen \& Walp. ex Meyen, Ambrosia cumanensis Kunth. In: Paniagua-Zambrana NY, Bussmann RW 
(Eds.) Ethnobotany of Mountain Regions - Ethnobotany of the Andes. Springer International Publishing, Cham. doi: 10.1007/978-3-319-77093-2_21-1

Paniagua-Zambrana NY, Bussmann RW, Echeverría J, Romero C. 2020. Artemisia absinthium L., Artemisia abrotanum L., Artemisia annua L., Artemisia copa Phil. In: Paniagua-Zambrana NY, Bussmann RW (Eds.) Ethnobotany of Mountain Regions - Ethnobotany of the Andes. Springer International Publishing, Cham. doi: 10.1007/978-3-319-77093-2_28-1

Paniagua-Zambrana NY, Bussmann RW, Echeverría J, Romero C. 2020. Baccharis alnifolia Meyen \& Walp., Baccharis boliviensis (Wedd.) Cabrera, Baccharis caespitosa (Ruiz \& Pav.) Pers., Baccharis calliprinos Griseb., Baccharis ciliata Gardner, Baccharis indica L., Baccharis juncea (Cass.) Desf., Baccharis odorata Kunth, Baccharis pentlandii DC., Baccharis salicifolia (Ruiz \& Pav.) Pers., Baccharis santelicis Phil., Baccharis tola Phil., Baccharis vaccinioides Kunth. In: Paniagua-Zambrana NY, Bussmann RW (Eds.) Ethnobotany of Mountain Regions - Ethnobotany of the Andes. Springer International Publishing, Cham. doi: 10.1007/978-3-319-77093-2_33-1

Paniagua-Zambrana NY, Bussmann RW, Echeverría J, Romero C. 2020. Bidens laevis (L.) Britton, Stern \& Poggenb., Bidens pilosa L., Bidens pseudocosmos Sherff, Bidens sp. In: Paniagua-Zambrana NY, Bussmann RW (Eds.) Ethnobotany of Mountain Regions - Ethnobotany of the Andes. Springer International Publishing, Cham. doi: 10.1007/978-3-319-77093-2_38-1

Paniagua-Zambrana NY, Bussmann RW, Echeverría J, Romero C. 2020. Chenopodium album L., Chenopodium hircinum Schrad. ssp. hircinum, Chenopodiastrum murale (L.) S. Fuentes, Uotila \& Borsch, Chenopodium quinoa Willd. In: Paniagua-Zambrana NY, Bussmann RW (Eds.) Ethnobotany of Mountain Regions - Ethnobotany of the Andes. Springer International Publishing, Cham. doi: 10.1007/978-3-319-77093-2_67-1

Paniagua-Zambrana NY, Bussmann RW, Echeverría J, Romero C. 2020. Chuquiraga atacamensis Kuntze, Chuquiraga jussieui J.F. Gmel., Chuquiraga spinosa Less., Chuquiraga weberbaueri Tovar. In: Paniagua-Zambrana NY, Bussmann RW (Eds.) Ethnobotany of Mountain Regions - Ethnobotany of the Andes. Springer International Publishing, Cham. doi: 10.1007/978-3-319-77093-2_70-2

Paniagua-Zambrana NY, Bussmann RW, Echeverría J, Romero C. 2020. Conyza bonariensis (L.) Cronquist, Conyza deserticola Phil. In: Paniagua-Zambrana NY, Bussmann RW (Eds.) Ethnobotany of Mountain Regions - Ethnobotany of the Andes. Springer International Publishing, Cham. doi: 10.1007/978-3-319-77093-2_77-1

Paniagua-Zambrana NY, Bussmann RW, Echeverría J, Romero C. 2020. Matricaria chamomilla L., Matricaria discoidea DC. In: Paniagua-Zambrana NY, Bussmann RW (Eds.) Ethnobotany of Mountain Regions - Ethnobotany of the Andes. Springer International Publishing, Cham. doi: 10.1007/978-3-319-77093-2_183-1

Paniagua-Zambrana NY, Bussmann RW, Echeverría J, Romero C. 2020. Mentax piperita L., Mentha spicata L., Mentha suaveolens Ehrh. In: Paniagua-Zambrana NY, Bussmann RW (Eds.) Ethnobotany of Mountain Regions - Ethnobotany of the Andes. Springer International Publishing, Cham. doi: 10.1007/978-3-319-77093-2_189-1

Paniagua-Zambrana NY, Bussmann RW, Echeverría J, Romero C. 2020. Plantago australis Lam., Plantago lanceolata L., Plantago major L., Plantago rancaguae Steud., Plantago sericea Ruiz \& Pav. In: Paniagua-Zambrana NY, Bussmann RW (Eds.) Ethnobotany of Mountain Regions - Ethnobotany of the Andes. Springer International Publishing, Cham. doi: 10.1007/978-3-319-77093-2_237-1

Paniagua-Zambrana NY, Bussmann RW, Echeverría J, Romero C. 2020. Solanum albidum Dunal, Solanum americanum Mill., Solanum fragile Wedd., Solanum herbabona Reiche, Solanum lyciopersicum L. Solanum mammosum L., Solanum marginatum L. f., Solanum nigrum L., Solanum nitidum Ruiz. \& Pav., Solanum nudum Dunal. In: Paniagua-Zambrana NY, Bussmann RW (Eds.) Ethnobotany of Mountain Regions - Ethnobotany of the Andes. Springer International Publishing, Cham. doi: 10.1007/978-3-319-77093-2_270-1

Paniagua-Zambrana NY, Bussmann RW, Echeverría J, Romero C. 2020. Tagetes elliptica Sm., Tagetes erecta L., Tagetes minuta L., Tagetes multiflora Kunth. In: Paniagua-Zambrana NY, Bussmann RW (Eds.) Ethnobotany of Mountain Regions - Ethnobotany of the Andes. Springer International Publishing, Cham. doi: 10.1007/978-3-31977093-2_276-1

Paniagua-Zambrana NY, Bussmann RW, Echeverría J, Romero C. 2020. Tessaria absinthioides (Hook. \& Arn.) DC., Tessaria integrifolia Ruiz \& Pav. In: Paniagua-Zambrana NY, Bussmann RW (Eds.) Ethnobotany of Mountain Regions - Ethnobotany of the Andes. Springer International Publishing, Cham. doi: 10.1007/978-3-319-77093-2_281-1 
Paniagua-Zambrana NY, Bussmann RW, Echeverría J, Romero C. 2020. Urtica dioica L., Urtica echinata Benth., Urtica magellanica Juss. ex Poir., Urtica urens L. In: Paniagua-Zambrana NY, Bussmann RW (Eds.) Ethnobotany of Mountain Regions - Ethnobotany of the Andes. Springer International Publishing, Cham. doi: 10.1007/978-3-319-77093-2_293 doi: 10.1007/978-3-319-77093-2_47-1-1

Paniagua-Zambrana NY, Bussmann RW, Echeverría J, Romero C. 2020. Valeriana convallarioides (Schmale) B.B. Larsen, Valeriana decussata Ruiz \& Pav., Valeriana microphylla Kunth, Valeriana micropterina Wedd., Valeriana nivalis Wedd., Valeriana officinalis L., Valeriana plantaginea Kunth, Valeriana rigida Ruiz \& Pav., Valeriana scandens L., Valeriana urbanii Phil. In: Paniagua-Zambrana NY, Bussmann RW (Eds.) Ethnobotany of Mountain Regions Ethnobotany of the Andes. Springer International Publishing, Cham. doi: 10.1007/978-3-319-77093-2_294-1

Paniagua-Zambrana NY, Bussmann RW, Echeverría J, Romero C. 2020. Verbena bonariensis L., Verbena litoralis Kunth, Verbena officinalis L. In: Paniagua-Zambrana NY, Bussmann RW (Eds.) Ethnobotany of Mountain Regions Ethnobotany of the Andes. Springer International Publishing, Cham. doi: 10.1007/978-3-319-77093-2_295-1

Paniagua-Zambrana NY, Bussmann RW, Echeverría, J 2020. Ephedra americana Humb. \& Bonpl. ex Willd., Ephedra breana Phil., Ephedra multiflora Phil. ex Stapf, Ephedra rupestris Benth. In: Paniagua-Zambrana NY, Bussmann RW (Eds.) Ethnobotany of Mountain Regions - Ethnobotany of the Andes. Springer International Publishing, Cham. doi: 10.1007/978-3-319-77093-2_109-1

Paniagua-Zambrana NY, Bussmann RW, Echeverría, J 2020. Flaveria bidentis (L.) Kuntze. In: Paniagua-Zambrana NY, Bussmann RW (Eds.) Ethnobotany of Mountain Regions - Ethnobotany of the Andes. Springer International Publishing, Cham. doi: 10.1007/978-3-319-77093-2_123-2

Paniagua-Zambrana NY, Bussmann RW, Echeverría, J 2020. Galium hypocarpium (L.) Endl. ex Griseb., Galium sp., Relbunium sp. In: Paniagua-Zambrana NY, Bussmann RW (Eds.) Ethnobotany of Mountain Regions - Ethnobotany of the Andes. Springer International Publishing, Cham. doi: 10.1007/978-3-319-77093-2_126-1

Paniagua-Zambrana NY, Bussmann RW, Echeverría,J. 2020. Calceolaria bartsiifolia Wedd., Calceolaria buchtieniana Kraenzl., Calceolaria engleriana Kraenzl., Calceolaria inamoena Kraenzl., Calceolaria rugulosa Edwin, Calceolaria stellarïfolia Phil. In: Paniagua-Zambrana NY, Bussmann RW (Eds.) Ethnobotany of Mountain Regions - Ethnobotany of the Andes. Springer International Publishing, Cham. doi: 10.1007/978-3-319-77093-2_50-2

Paniagua-Zambrana NY, Bussmann RW, Echeverría,J. 2020. Ethnobotany of Mountain Regions - Ethnobotany of the Andes - Bolivia, Peru, Chile. In: Paniagua-Zambrana NY, Bussmann RW (Eds.) Ethnobotany of Mountain Regions - Ethnobotany of the Andes. Springer International Publishing, Cham. doi: 10.1007/978-3-319-77093-2_2-2

Paniagua-Zambrana NY, Bussmann RW, Echeverría,J. 2020. Lepidium bonariense L., Lepidium virginicum L. In: Paniagua-Zambrana NY, Bussmann RW (Eds.) Ethnobotany of Mountain Regions - Ethnobotany of the Andes. Springer International Publishing, Cham. doi: 10.1007/978-3-319-77093-2_166-1

Paniagua-Zambrana NY, Bussmann RW, Echeverría,J. 2020. Mutisia acuminata Ruiz \& Pav., Mutisia hamata Reiche, Mutisia lanigera Wedd., Mutisia orbygniana Wedd. In: Paniagua-Zambrana NY, Bussmann RW (Eds.) Ethnobotany of Mountain Regions - Ethnobotany of the Andes. Springer International Publishing, Cham. doi: 10.1007/978-3-31977093-2_196-1

Paniagua-Zambrana NY, Bussmann RW, Echeverría,J. 2020. Perezia atacamensis (Phil.) Reiche, Perezia pungens (Bonpl.) Less. In: Paniagua-Zambrana NY, Bussmann RW (Eds.) Ethnobotany of Mountain Regions - Ethnobotany of the Andes. Springer International Publishing, Cham.

Paniagua-Zambrana NY, Bussmann RW, Echeverría,J. 2020. Polylepis pacensis M. Kessler \& Schmidt-Leb., Polylepis racemosa Ruiz \& Pav., Polylepis tarapacana Phil., Polylepis tomentella Wedd. In: Paniagua-Zambrana NY, Bussmann RW (Eds.) Ethnobotany of Mountain Regions - Ethnobotany of the Andes. Springer International Publishing, Cham. doi: 10.1007/978-3-319-77093-2_239-1

Paniagua-Zambrana NY, Bussmann RW, Echeverría,J. 2020. Prosopis alba Griseb. var. alba, Prosopis laevigata (Humb. \& Bonpl. ex Willd.) M.C., Prosopis pallida (Humb. \& Bonpl. ex Willd.) Kunth. In: Paniagua-Zambrana NY, Bussmann RW (Eds.) Ethnobotany of Mountain Regions - Ethnobotany of the Andes. Springer International Publishing, Cham. doi: 10.1007/978-3-319-77093-2_242-1

Paniagua-Zambrana NY, Bussmann RW, Echeverría,J. 2020. Rumex acetosella L., Rumex crispus L., Rumex cuneifolius Campd. In: Paniagua-Zambrana NY, Bussmann RW (Eds.) Ethnobotany of Mountain Regions Ethnobotany of the Andes. Springer International Publishing, Cham. doi: 10.1007/978-3-319-77093-2_255-1 
Paniagua-Zambrana NY, Bussmann RW, Echeverría,J. 2020. Schinus areira L., Schinus molle L. In: PaniaguaZambrana NY, Bussmann RW (Eds.) Ethnobotany of Mountain Regions - Ethnobotany of the Andes. Springer International Publishing, Cham. doi: 10.1007/978-3-319-77093-2_262-1

Paniagua-Zambrana NY, Bussmann RW, Echeverría,J. 2020. Schkuhria pinnata (Lam.) Kuntze ex Thell. In: PaniaguaZambrana NY, Bussmann RW (Eds.) Ethnobotany of Mountain Regions - Ethnobotany of the Andes. Springer International Publishing, Cham. doi: 10.1007/978-3-319-77093-2_263-1

Paniagua-Zambrana NY, Bussmann RW, Echeverría,J. 2020. Werneria aretioides Wedd., Werneria glaberrima Phil., Werneria heteroloba Wedd., Werneria nubigena Kunth, Werneria pumila Kunth, Werneria pygmaea Gillies ex Hook. \& Arn., Xenophyllum ciliolatum (A. Gray) V.A. Funk, Xenophyllum humile (Kunth) V.A. Funk, Xenophyllum incisum (Phil.) V.A. Funk, Xenophyllum poposum (Phil.) V.A. Funk, Xenophyllum weddellii (Phil.) V.A. Funk. In: PaniaguaZambrana NY, Bussmann RW (Eds.) Ethnobotany of Mountain Regions - Ethnobotany of the Andes. Springer International Publishing, Cham. doi: 10.1007/978-3-319-77093-2_299-1

Paniagua-Zambrana NY, Bussmann RW, Romero C 2020. Cassia occidentalis L. In: Paniagua-Zambrana NY, Bussmann RW (Eds.) Ethnobotany of Mountain Regions - Ethnobotany of the Andes. Springer International Publishing, Cham. doi: 10.1007/978-3-319-77093-2_58-1

Paniagua-Zambrana NY, Bussmann RW, Romero C, Echeverría J. 2020. Baccharis genistelloides (Lam.) Pers. In: Paniagua-Zambrana NY, Bussmann RW (Eds.) Ethnobotany of Mountain Regions - Ethnobotany of the Andes. Springer International Publishing, Cham. doi: 10.1007/978-3-319-77093-2_304-1

Paniagua-Zambrana NY, Bussmann RW, Romero C, Echeverría J. 2020. Baccharis latifolia (Ruiz. \& Pav.) Pers. In: Paniagua-Zambrana NY, Bussmann RW (Eds.) Ethnobotany of Mountain Regions - Ethnobotany of the Andes. Springer International Publishing, Cham. doi: 10.1007/978-3-319-77093-2_305-1

Paniagua-Zambrana NY, Bussmann RW, Romero C. 2020. Achillea millefolium L. In: Paniagua-Zambrana NY, Bussmann RW (Eds.) Ethnobotany of Mountain Regions - Ethnobotany of the Andes. Springer International Publishing, Cham. doi: 10.1007/978-3-319-77093-2_6-1

Paniagua-Zambrana NY, Bussmann RW, Romero C. 2020. Achyrocline bogotensis (Kunth) DC., Achyrocline satureioides (Lam.) DC. In: Paniagua-Zambrana NY, Bussmann RW (Eds.) Ethnobotany of Mountain Regions Ethnobotany of the Andes. Springer International Publishing, Cham. doi: 10.1007/978-3-319-77093-2_7-1

Paniagua-Zambrana NY, Bussmann RW, Romero C. 2020. Adiantum concinnum Humb. \& Bonpl. ex Willd., Addiantum raddianum C. Presl. In: Paniagua-Zambrana NY, Bussmann RW (Eds.) Ethnobotany of Mountain Regions - Ethnobotany of the Andes. Springer International Publishing, Cham. doi: 10.1007/978-3-319-77093-2_10-1

Paniagua-Zambrana NY, Bussmann RW, Romero C. 2020. Agave americana L. In: Paniagua-Zambrana NY, Bussmann RW (Eds.) Ethnobotany of Mountain Regions - Ethnobotany of the Andes. Springer International Publishing, Cham. doi: 10.1007/978-3-319-77093-2_11-1

Paniagua-Zambrana NY, Bussmann RW, Romero C. 2020. Ageratum conyzioides L. In: Paniagua-Zambrana NY, Bussmann RW (Eds.) Ethnobotany of Mountain Regions - Ethnobotany of the Andes. Springer International Publishing, Cham. doi: 10.1007/978-3-319-77093-2_12-1

Paniagua-Zambrana NY, Bussmann RW, Romero C. 2020. Allium cepa L., Allium fistulosum L., Allium ramosum L., Allium sativum L. In: Paniagua-Zambrana NY, Bussmann RW (Eds.) Ethnobotany of Mountain Regions - Ethnobotany of the Andes. Springer International Publishing, Cham. doi: 10.1007/978-3-319-77093-2_15-1

Paniagua-Zambrana NY, Bussmann RW, Romero C. 2020. Alnus acuminata Kunth. In: Paniagua-Zambrana NY, Bussmann RW (Eds.) Ethnobotany of Mountain Regions - Ethnobotany of the Andes. Springer International Publishing, Cham. doi: 10.1007/978-3-319-77093-2_16-1

Paniagua-Zambrana NY, Bussmann RW, Romero C. 2020. Aloe vera (L.) Burm. f. In: Paniagua-Zambrana NY, Bussmann RW (Eds.) Ethnobotany of Mountain Regions - Ethnobotany of the Andes. Springer International Publishing, Cham. doi: 10.1007/978-3-319-77093-2_17-1

Paniagua-Zambrana NY, Bussmann RW, Romero C. 2020. Alternanthera brasiliana (L.) Kuntze, Alternanthera halimifolia (Lam.) Standl. ex Pittier, Alternanthera porrigens (Jacq.) Kuntze, Alternanthera villosa Kunth. In: PaniaguaZambrana NY, Bussmann RW (Eds.) Ethnobotany of Mountain Regions - Ethnobotany of the Andes. Springer International Publishing, Cham. doi: 10.1007/978-3-319-77093-2_19-1 
Paniagua-Zambrana NY, Bussmann RW, Romero C. 2020. Anacardium occidentale L. In: Paniagua-Zambrana NY, Bussmann RW (Eds.) Ethnobotany of Mountain Regions - Ethnobotany of the Andes. Springer International Publishing, Cham. doi: 10.1007/978-3-319-77093-2_22-1

Paniagua-Zambrana NY, Bussmann RW, Romero C. 2020. Anethum graveolens L. In: Paniagua-Zambrana NY, Bussmann RW (Eds.) Ethnobotany of Mountain Regions - Ethnobotany of the Andes. Springer International Publishing, Cham. doi: 10.1007/978-3-319-77093-2_23-1

Paniagua-Zambrana NY, Bussmann RW, Romero C. 2020. Argemone mexicana L. In: Paniagua-Zambrana NY, Bussmann RW (Eds.) Ethnobotany of Mountain Regions - Ethnobotany of the Andes. Springer International Publishing, Cham. doi: 10.1007/978-3-319-77093-2_26-1

Paniagua-Zambrana NY, Bussmann RW, Romero C. 2020. Avena sativa L. In: Paniagua-Zambrana NY, Bussmann RW (Eds.) Ethnobotany of Mountain Regions - Ethnobotany of the Andes. Springer International Publishing, Cham. doi: 10.1007/978-3-319-77093-2_31-1

Paniagua-Zambrana NY, Bussmann RW, Romero C. 2020. Bactris gasipaes Kunth. In: Paniagua-Zambrana NY, Bussmann RW (Eds.) Ethnobotany of Mountain Regions - Ethnobotany of the Andes. Springer International Publishing, Cham. doi: 10.1007/978-3-319-77093-2_34-1

Paniagua-Zambrana NY, Bussmann RW, Romero C. 2020. Bauhinia variegata L. In: Paniagua-Zambrana NY, Bussmann RW (Eds.) Ethnobotany of Mountain Regions - Ethnobotany of the Andes. Springer International Publishing, Cham. doi: 10.1007/978-3-319-77093-2_35-1

Paniagua-Zambrana NY, Bussmann RW, Romero C. 2020. Berberis rigidifolia Kunth ex DC. In: Paniagua-Zambrana NY, Bussmann RW (Eds.) Ethnobotany of Mountain Regions - Ethnobotany of the Andes. Springer International Publishing, Cham. doi: 10.1007/978-3-319-77093-2_37-1

Paniagua-Zambrana NY, Bussmann RW, Romero C. 2020. Bixa orellana L. In: Paniagua-Zambrana NY, Bussmann RW (Eds.) Ethnobotany of Mountain Regions - Ethnobotany of the Andes. Springer International Publishing, Cham. doi: 10.1007/978-3-319-77093-2_39-1

Paniagua-Zambrana NY, Bussmann RW, Romero C. 2020. Borago officinalis L. In: Paniagua-Zambrana NY, Bussmann RW (Eds.) Ethnobotany of Mountain Regions - Ethnobotany of the Andes. Springer International Publishing, Cham. doi: 10.1007/978-3-319-77093-2_40-1

Paniagua-Zambrana NY, Bussmann RW, Romero C. 2020. Brassica oleracea L. In: Paniagua-Zambrana NY, Bussmann RW (Eds.) Ethnobotany of Mountain Regions - Ethnobotany of the Andes. Springer International Publishing, Cham. doi: 10.1007/978-3-319-77093-2_41-1

Paniagua-Zambrana NY, Bussmann RW, Romero C. 2020. Brosimum rubescens Taub. In: Paniagua-Zambrana NY, Bussmann RW (Eds.) Ethnobotany of Mountain Regions - Ethnobotany of the Andes. Springer International Publishing, Cham. doi: 10.1007/978-3-319-77093-2_42-1

Paniagua-Zambrana NY, Bussmann RW, Romero C. 2020. Brugmansia candida Pers., Brugmanisa sanguinea (Ruiz \& Pav.) D. Don. In: Paniagua-Zambrana NY, Bussmann RW (Eds.) Ethnobotany of Mountain Regions - Ethnobotany of the Andes. Springer International Publishing, Cham. doi: 10.1007/978-3-319-77093-2_44-1

Paniagua-Zambrana NY, Bussmann RW, Romero C. 2020. Bursera graveolens (Kunth.) Triana \& Planch. In: Paniagua-Zambrana NY, Bussmann RW (Eds.) Ethnobotany of Mountain Regions - Ethnobotany of the Andes. Springer International Publishing, Cham. doi: 10.1007/978-3-319-77093-2_47-1

Paniagua-Zambrana NY, Bussmann RW, Romero C. 2020. Caesalpinia paipai Ruiz \& Pav., Caesalpinia spinosa (Molina) Kuntze. In: Paniagua-Zambrana NY, Bussmann RW (Eds.) Ethnobotany of Mountain Regions - Ethnobotany of the Andes. Springer International Publishing, Cham. doi: 10.1007/978-3-319-77093-2_48-1

Paniagua-Zambrana NY, Bussmann RW, Romero C. 2020. Calendula officinalis L. In: Paniagua-Zambrana NY, Bussmann RW (Eds.) Ethnobotany of Mountain Regions - Ethnobotany of the Andes. Springer International Publishing, Cham. doi: 10.1007/978-3-319-77093-2_51-1

Paniagua-Zambrana NY, Bussmann RW, Romero C. 2020. Capsella bursa-pastoris (L.) Medik. In: PaniaguaZambrana NY, Bussmann RW (Eds.) Ethnobotany of Mountain Regions - Ethnobotany of the Andes. Springer International Publishing, Cham. doi: 10.1007/978-3-319-77093-2_54-1 
Paniagua-Zambrana NY, Bussmann RW, Romero C. 2020. Capsicum annuum L. In: Paniagua-Zambrana NY, Bussmann RW (Eds.) Ethnobotany of Mountain Regions - Ethnobotany of the Andes. Springer International Publishing, Cham. doi: 10.1007/978-3-319-77093-2_56-1

Paniagua-Zambrana NY, Bussmann RW, Romero C. 2020. Carica papaya L., Vasconcellea pubescens A. DC. In: Paniagua-Zambrana NY, Bussmann RW (Eds.) Ethnobotany of Mountain Regions - Ethnobotany of the Andes. Springer International Publishing, Cham. doi: 10.1007/978-3-319-77093-2_57-1

Paniagua-Zambrana NY, Bussmann RW, Romero C. 2020. Cecropia andina Cuatrec., Cecropia mutisiana Mildbr., Cecropia peltata L. In: Paniagua-Zambrana NY, Bussmann RW (Eds.) Ethnobotany of Mountain Regions Ethnobotany of the Andes. Springer International Publishing, Cham. doi: 10.1007/978-3-319-77093-2_59-1

Paniagua-Zambrana NY, Bussmann RW, Romero C. 2020. Centella asiatica (L.) Urb. In: Paniagua-Zambrana NY, Bussmann RW (Eds.) Ethnobotany of Mountain Regions - Ethnobotany of the Andes. Springer International Publishing, Cham. doi: 10.1007/978-3-319-77093-2_61-1

Paniagua-Zambrana NY, Bussmann RW, Romero C. 2020. Cestrum parqui L'Hér., Cestrum buxifolium Kunth, Cestrum sp. In: Paniagua-Zambrana NY, Bussmann RW (Eds.) Ethnobotany of Mountain Regions - Ethnobotany of the Andes. Springer International Publishing, Cham. doi: 10.1007/978-3-319-77093-2_63-

Paniagua-Zambrana NY, Bussmann RW, Romero C. 2020. Cetraria islandica (L.) Ach. In: Paniagua-Zambrana NY, Bussmann RW (Eds.) Ethnobotany of Mountain Regions - Ethnobotany of the Andes. Springer International Publishing, Cham. doi: 10.1007/978-3-319-77093-2_64-1

Paniagua-Zambrana NY, Bussmann RW, Romero C. 2020. Cicer arietinum L. In: Paniagua-Zambrana NY, Bussmann RW (Eds.) Ethnobotany of Mountain Regions - Ethnobotany of the Andes. Springer International Publishing, Cham. doi: 10.1007/978-3-319-77093-2_71-1

Paniagua-Zambrana NY, Bussmann RW, Romero C. 2020. Cinchona pubescens Vahl, Cinchona officinalis L. In: Paniagua-Zambrana NY, Bussmann RW (Eds.) Ethnobotany of Mountain Regions - Ethnobotany of the Andes. Springer International Publishing, Cham. doi: 10.1007/978-3-319-77093-2_72-1

Paniagua-Zambrana NY, Bussmann RW, Romero C. 2020. Citrus maxima (L.) Osbeck, Citrus medica L., Citrus reticulata Blanco. In: Paniagua-Zambrana NY, Bussmann RW (Eds.) Ethnobotany of Mountain Regions - Ethnobotany of the Andes. Springer International Publishing, Cham. doi: 10.1007/978-3-319-77093-2_74-1

Paniagua-Zambrana NY, Bussmann RW, Romero C. 2020. Coffea arabica L. In: Paniagua-Zambrana NY, Bussmann RW (Eds.) Ethnobotany of Mountain Regions - Ethnobotany of the Andes. Springer International Publishing, Cham. doi: 10.1007/978-3-319-77093-2_76-1

Paniagua-Zambrana NY, Bussmann RW, Romero C. 2020. Cordia alliodora (Ruiz \& Pav.) Oken, Cordia lutea Lam. In: Paniagua-Zambrana NY, Bussmann RW (Eds.) Ethnobotany of Mountain Regions - Ethnobotany of the Andes. Springer International Publishing, Cham. doi: 10.1007/978-3-319-77093-2_78-1

Paniagua-Zambrana NY, Bussmann RW, Romero C. 2020. Coriandrum sativum L. In: Paniagua-Zambrana NY, Bussmann RW (Eds.) Ethnobotany of Mountain Regions - Ethnobotany of the Andes. Springer International Publishing, Cham. doi: 10.1007/978-3-319-77093-2_79-2

Paniagua-Zambrana NY, Bussmann RW, Romero C. 2020. Crescentia cujete L. In: Paniagua-Zambrana NY, Bussmann RW (Eds.) Ethnobotany of Mountain Regions - Ethnobotany of the Andes. Springer International Publishing, Cham. doi: 10.1007/978-3-319-77093-2_83-1

Paniagua-Zambrana NY, Bussmann RW, Romero C. 2020. Croton draconoides Müll. Arg., Croton lechleri Müll. Arg. In: Paniagua-Zambrana NY, Bussmann RW (Eds.) Ethnobotany of Mountain Regions - Ethnobotany of the Andes. Springer International Publishing, Cham. doi: 10.1007/978-3-319-77093-2_84-1

Paniagua-Zambrana NY, Bussmann RW, Romero C. 2020. Cucumis sativus L. In: Paniagua-Zambrana NY, Bussmann RW (Eds.) Ethnobotany of Mountain Regions - Ethnobotany of the Andes. Springer International Publishing, Cham. doi: 10.1007/978-3-319-77093-2_87-1

Paniagua-Zambrana NY, Bussmann RW, Romero C. 2020. Cucurbita maxima Duchesne, Cucurbita moschata Duchesne. In: Paniagua-Zambrana NY, Bussmann RW (Eds.) Ethnobotany of Mountain Regions - Ethnobotany of the Andes. Springer International Publishing, Cham. doi: 10.1007/978-3-319-77093-2_88-1 
Paniagua-Zambrana NY, Bussmann RW, Romero C. 2020. Cydonia oblonga Mill. In: Paniagua-Zambrana NY, Bussmann RW (Eds.) Ethnobotany of Mountain Regions - Ethnobotany of the Andes. Springer International Publishing, Cham. doi: 10.1007/978-3-319-77093-2_92-1

Paniagua-Zambrana NY, Bussmann RW, Romero C. 2020. Cymbopogon citratus (DC.) Stapf. In: Paniagua-Zambrana NY, Bussmann RW (Eds.) Ethnobotany of Mountain Regions - Ethnobotany of the Andes. Springer International Publishing, Cham. doi: 10.1007/978-3-319-77093-2_93-1

Paniagua-Zambrana NY, Bussmann RW, Romero C. 2020. Cynara cardunculus L. In: Paniagua-Zambrana NY, Bussmann RW (Eds.) Ethnobotany of Mountain Regions - Ethnobotany of the Andes. Springer International Publishing, Cham. doi: 10.1007/978-3-319-77093-2_94-1

Paniagua-Zambrana NY, Bussmann RW, Romero C. 2020. Daucus carota L., Daucus montanus Humb. \& Bonpl. ex Spreng. In: Paniagua-Zambrana NY, Bussmann RW (Eds.) Ethnobotany of Mountain Regions - Ethnobotany of the Andes. Springer International Publishing, Cham. doi: 10.1007/978-3-319-77093-2_95-1

Paniagua-Zambrana NY, Bussmann RW, Romero C. 2020. Desmodium molliculum (Kunth) DC., Desmosium triflorum (L.) DC. In: Paniagua-Zambrana NY, Bussmann RW (Eds.) Ethnobotany of Mountain Regions - Ethnobotany of the Andes. Springer International Publishing, Cham. doi: 10.1007/978-3-319-77093-2_97-1

Paniagua-Zambrana NY, Bussmann RW, Romero C. 2020. Dianthus caryophyllus L., Dianthus plumarius L. In: Paniagua-Zambrana NY, Bussmann RW (Eds.) Ethnobotany of Mountain Regions - Ethnobotany of the Andes. Springer International Publishing, Cham. doi: 10.1007/978-3-319-77093-2_98-1

Paniagua-Zambrana NY, Bussmann RW, Romero C. 2020. Dioscorea tambillensis R. Knuth, Dioscorea trifida L. f. In: Paniagua-Zambrana NY, Bussmann RW (Eds.) Ethnobotany of Mountain Regions - Ethnobotany of the Andes. Springer International Publishing, Cham. doi: 10.1007/978-3-319-77093-2_101-1

Paniagua-Zambrana NY, Bussmann RW, Romero C. 2020. Dodonaea viscosa Jacq. In: Paniagua-Zambrana NY, Bussmann RW (Eds.) Ethnobotany of Mountain Regions - Ethnobotany of the Andes. Springer International Publishing, Cham. doi: 10.1007/978-3-319-77093-2_103-1

Paniagua-Zambrana NY, Bussmann RW, Romero C. 2020. Drimys granadensis L. f. In: Paniagua-Zambrana NY, Bussmann RW (Eds.) Ethnobotany of Mountain Regions - Ethnobotany of the Andes. Springer International Publishing, Cham. doi: 10.1007/978-3-319-77093-2_104-1

Paniagua-Zambrana NY, Bussmann RW, Romero C. 2020. Dysphania ambrosioides (L.) Mosyakin \& Clemants. In: Paniagua-Zambrana NY, Bussmann RW (Eds.) Ethnobotany of Mountain Regions - Ethnobotany of the Andes. Springer International Publishing, Cham. doi: 10.1007/978-3-319-77093-2_106-1

Paniagua-Zambrana NY, Bussmann RW, Romero C. 2020. Elatteria cardamomum(L.) Maton. In: Paniagua-Zambrana NY, Bussmann RW (Eds.) Ethnobotany of Mountain Regions - Ethnobotany of the Andes. Springer International Publishing, Cham. doi: 10.1007/978-3-319-77093-2_108-1

Paniagua-Zambrana NY, Bussmann RW, Romero C. 2020. Equisetum bogotense Kunth, Equisetum giganteum L. In: Paniagua-Zambrana NY, Bussmann RW (Eds.) Ethnobotany of Mountain Regions - Ethnobotany of the Andes. Springer International Publishing, Cham. doi: 10.1007/978-3-319-77093-2_110-1

Paniagua-Zambrana NY, Bussmann RW, Romero C. 2020. Eriobotrya japonica (Thunb.) Lindl. In: PaniaguaZambrana NY, Bussmann RW (Eds.) Ethnobotany of Mountain Regions - Ethnobotany of the Andes. Springer International Publishing, Cham. doi: 10.1007/978-3-319-77093-2_112-1

Paniagua-Zambrana NY, Bussmann RW, Romero C. 2020. Eryngium foetidum L. In: Paniagua-Zambrana NY, Bussmann RW (Eds.) Ethnobotany of Mountain Regions - Ethnobotany of the Andes. Springer International Publishing, Cham. doi: 10.1007/978-3-319-77093-2_114-1

Paniagua-Zambrana NY, Bussmann RW, Romero C. 2020. Erythrina fusca Lour. In: Paniagua-Zambrana NY, Bussmann RW (Eds.) Ethnobotany of Mountain Regions - Ethnobotany of the Andes. Springer International Publishing, Cham. doi: 10.1007/978-3-319-77093-2_115-1

Paniagua-Zambrana NY, Bussmann RW, Romero C. 2020. Erythroxylum coca Lam. In: Paniagua-Zambrana NY, Bussmann RW (Eds.) Ethnobotany of Mountain Regions - Ethnobotany of the Andes. Springer International Publishing, Cham. doi: 10.1007/978-3-319-77093-2_116-1 
Paniagua-Zambrana NY, Bussmann RW, Romero C. 2020. Eucalyptus citriodora Hook., Eucalyptus globulus Labill. In: Paniagua-Zambrana NY, Bussmann RW (Eds.) Ethnobotany of Mountain Regions - Ethnobotany of the Andes. Springer International Publishing, Cham. doi: 10.1007/978-3-319-77093-2_118-1

Paniagua-Zambrana NY, Bussmann RW, Romero C. 2020. Ficus carica L., Ficus insipida Willd. In: PaniaguaZambrana NY, Bussmann RW (Eds.) Ethnobotany of Mountain Regions - Ethnobotany of the Andes. Springer International Publishing, Cham. doi: 10.1007/978-3-319-77093-2_122-1

Paniagua-Zambrana NY, Bussmann RW, Romero C. 2020. Foeniculum vulgare Mill. In: Paniagua-Zambrana NY, Bussmann RW (Eds.) Ethnobotany of Mountain Regions - Ethnobotany of the Andes. Springer International Publishing, Cham. doi: 10.1007/978-3-319-77093-2_124-1

Paniagua-Zambrana NY, Bussmann RW, Romero C. 2020. Fragaria vesca L. In: Paniagua-Zambrana NY, Bussmann RW (Eds.) Ethnobotany of Mountain Regions - Ethnobotany of the Andes. Springer International Publishing, Cham. doi: 10.1007/978-3-319-77093-2_125-1

Paniagua-Zambrana NY, Bussmann RW, Romero C. 2020. Gliricidia sepium (Jacq.) Kunth ex Walp. In: PaniaguaZambrana NY, Bussmann RW (Eds.) Ethnobotany of Mountain Regions - Ethnobotany of the Andes. Springer International Publishing, Cham. doi: 10.1007/978-3-319-77093-2_131-1

Paniagua-Zambrana NY, Bussmann RW, Romero C. 2020. Glycine max (L.) Merr. In: Paniagua-Zambrana NY, Bussmann RW (Eds.) Ethnobotany of Mountain Regions - Ethnobotany of the Andes. Springer International Publishing, Cham. doi: 10.1007/978-3-319-77093-2_132-1

Paniagua-Zambrana NY, Bussmann RW, Romero C. 2020. Guazuma ulmifolia Lam. In: Paniagua-Zambrana NY, Bussmann RW (Eds.) Ethnobotany of Mountain Regions - Ethnobotany of the Andes. Springer International Publishing, Cham. doi: 10.1007/978-3-319-77093-2_134-1

Paniagua-Zambrana NY, Bussmann RW, Romero C. 2020. Hedera helix L. In: Paniagua-Zambrana NY, Bussmann RW (Eds.) Ethnobotany of Mountain Regions - Ethnobotany of the Andes. Springer International Publishing, Cham. doi: 10.1007/978-3-319-77093-2_138-1

Paniagua-Zambrana NY, Bussmann RW, Romero C. 2020. Hesperomeles obtusifolia (Pers.) Lindl. In: PaniaguaZambrana NY, Bussmann RW (Eds.) Ethnobotany of Mountain Regions - Ethnobotany of the Andes. Springer International Publishing, Cham. doi: 10.1007/978-3-319-77093-2_139-1

Paniagua-Zambrana NY, Bussmann RW, Romero C. 2020. Hordeum vulgare L. In: Paniagua-Zambrana NY, Bussmann RW (Eds.) Ethnobotany of Mountain Regions - Ethnobotany of the Andes. Springer International Publishing, Cham. doi: 10.1007/978-3-319-77093-2_141-1

Paniagua-Zambrana NY, Bussmann RW, Romero C. 2020. Hura crepitans L. In: Paniagua-Zambrana NY, Bussmann RW (Eds.) Ethnobotany of Mountain Regions - Ethnobotany of the Andes. Springer International Publishing, Cham. doi: 10.1007/978-3-319-77093-2_142-1

Paniagua-Zambrana NY, Bussmann RW, Romero C. 2020. Hymenea courbaril L. In: Paniagua-Zambrana NY, Bussmann RW (Eds.) Ethnobotany of Mountain Regions - Ethnobotany of the Andes. Springer International Publishing, Cham. doi: 10.1007/978-3-319-77093-2_143-1

Paniagua-Zambrana NY, Bussmann RW, Romero C. 2020. Hyptis capitata Jacq. In: Paniagua-Zambrana NY, Bussmann RW (Eds.) Ethnobotany of Mountain Regions - Ethnobotany of the Andes. Springer International Publishing, Cham. doi: 10.1007/978-3-319-77093-2_1346-1

Paniagua-Zambrana NY, Bussmann RW, Romero C. 2020. Ilex guayusa Loes. In: Paniagua-Zambrana NY, Bussmann RW (Eds.) Ethnobotany of Mountain Regions - Ethnobotany of the Andes. Springer International Publishing, Cham. doi: 10.1007/978-3-319-77093-2_147-1

Paniagua-Zambrana NY, Bussmann RW, Romero C. 2020. Inga edulis Mart. In: Paniagua-Zambrana NY, Bussmann RW (Eds.) Ethnobotany of Mountain Regions - Ethnobotany of the Andes. Springer International Publishing, Cham. doi: 10.1007/978-3-319-77093-2_148-1

Paniagua-Zambrana NY, Bussmann RW, Romero C. 2020. Ipomoea batatas (L.) Lam., Ipomoea pauciflora M. Martens \& Galeotti. In: Paniagua-Zambrana NY, Bussmann RW (Eds.) Ethnobotany of Mountain Regions - Ethnobotany of the Andes. Springer International Publishing, Cham. doi: 10.1007/978-3-319-77093-2_149-1 
Paniagua-Zambrana NY, Bussmann RW, Romero C. 2020. Iresine diffusa Humb. \& Bonpl. ex Willd., Iresine herbstii Hook. In: Paniagua-Zambrana NY, Bussmann RW (Eds.) Ethnobotany of Mountain Regions - Ethnobotany of the Andes. Springer International Publishing, Cham. doi: 10.1007/978-3-319-77093-2_150-1

Paniagua-Zambrana NY, Bussmann RW, Romero C. 2020. Jacaranda caucana Pittier. In: Paniagua-Zambrana NY, Bussmann RW (Eds.) Ethnobotany of Mountain Regions - Ethnobotany of the Andes. Springer International Publishing, Cham. doi: 10.1007/978-3-319-77093-2_152-1

Paniagua-Zambrana NY, Bussmann RW, Romero C. 2020. Jatropha curcas L., Jatropha gossypifolia L., Jatropha multifida L. In: Paniagua-Zambrana NY, Bussmann RW (Eds.) Ethnobotany of Mountain Regions - Ethnobotany of the Andes. Springer International Publishing, Cham. doi: 10.1007/978-3-319-77093-2_154-1

Paniagua-Zambrana NY, Bussmann RW, Romero C. 2020. Juglans boliviana (C. DC.) Dode, Juglans neotropica Diels. In: Paniagua-Zambrana NY, Bussmann RW (Eds.) Ethnobotany of Mountain Regions - Ethnobotany of the Andes. Springer International Publishing, Cham. doi: 10.1007/978-3-319-77093-2_155-1

Paniagua-Zambrana NY, Bussmann RW, Romero C. 2020. Kalanchoe pinnata (Lam.) Pers. In: Paniagua-Zambrana NY, Bussmann RW (Eds.) Ethnobotany of Mountain Regions - Ethnobotany of the Andes. Springer International Publishing, Cham. doi: 10.1007/978-3-319-77093-2_157-1

Paniagua-Zambrana NY, Bussmann RW, Romero C. 2020. Lachemilla pinnata (Ruiz \& Pav.) Rothm. In: PaniaguaZambrana NY, Bussmann RW (Eds.) Ethnobotany of Mountain Regions - Ethnobotany of the Andes. Springer International Publishing, Cham. doi: 10.1007/978-3-319-77093-2_159-1

Paniagua-Zambrana NY, Bussmann RW, Romero C. 2020. Lactuca sativa L. In: Paniagua-Zambrana NY, Bussmann RW (Eds.) Ethnobotany of Mountain Regions - Ethnobotany of the Andes. Springer International Publishing, Cham. doi: 10.1007/978-3-319-77093-2_160-1

Paniagua-Zambrana NY, Bussmann RW, Romero C. 2020. Lantana cujabensis Schauer. In: Paniagua-Zambrana NY, Bussmann RW (Eds.) Ethnobotany of Mountain Regions - Ethnobotany of the Andes. Springer International Publishing, Cham. doi: 10.1007/978-3-319-77093-2_162-1

Paniagua-Zambrana NY, Bussmann RW, Romero C. 2020. Lathyrus odoratus L. In: Paniagua-Zambrana NY, Bussmann RW (Eds.) Ethnobotany of Mountain Regions - Ethnobotany of the Andes. Springer International Publishing, Cham. doi: 10.1007/978-3-319-77093-2_163-1

Paniagua-Zambrana NY, Bussmann RW, Romero C. 2020. Lilium candidum L. In: Paniagua-Zambrana NY, Bussmann RW (Eds.) Ethnobotany of Mountain Regions - Ethnobotany of the Andes. Springer International Publishing, Cham. doi: 10.1007/978-3-319-77093-2_169-1

Paniagua-Zambrana NY, Bussmann RW, Romero C. 2020. Linum usitatissimum L. In: Paniagua-Zambrana NY, Bussmann RW (Eds.) Ethnobotany of Mountain Regions - Ethnobotany of the Andes. Springer International Publishing, Cham. doi: 10.1007/978-3-319-77093-2_171-1

Paniagua-Zambrana NY, Bussmann RW, Romero C. 2020. Lippia alba (Mill.) Britton \& P. Wilson. In: PaniaguaZambrana NY, Bussmann RW (Eds.) Ethnobotany of Mountain Regions - Ethnobotany of the Andes. Springer International Publishing, Cham. doi: 10.1007/978-3-319-77093-2_173-1

Paniagua-Zambrana NY, Bussmann RW, Romero C. 2020. Lupinus altimontanus C.P. Sm., Lupinus mutabilis Sweet. In: Paniagua-Zambrana NY, Bussmann RW (Eds.) Ethnobotany of Mountain Regions - Ethnobotany of the Andes. Springer International Publishing, Cham. doi: 10.1007/978-3-319-77093-2_176-2

Paniagua-Zambrana NY, Bussmann RW, Romero C. 2020. Lycopersicon esculentum Mill., Lycopersicon hirsutum Dunal, Lycopersicon peruvianum (L.) Mill. In: Paniagua-Zambrana NY, Bussmann RW (Eds.) Ethnobotany of Mountain Regions - Ethnobotany of the Andes. Springer International Publishing, Cham. doi: 10.1007/978-3-319-770932_177-1

Paniagua-Zambrana NY, Bussmann RW, Romero C. 2020. Malva parviflora L., Malva sylvestris L. In: PaniaguaZambrana NY, Bussmann RW (Eds.) Ethnobotany of Mountain Regions - Ethnobotany of the Andes. Springer International Publishing, Cham. doi: 10.1007/978-3-319-77093-2_179-1 
Paniagua-Zambrana NY, Bussmann RW, Romero C. 2020. Mangifera indica L. In: Paniagua-Zambrana NY, Bussmann RW (Eds.) Ethnobotany of Mountain Regions - Ethnobotany of the Andes. Springer International Publishing, Cham. doi: 10.1007/978-3-319-77093-2_180-1

Paniagua-Zambrana NY, Bussmann RW, Romero C. 2020. Manihot esculenta Crantz. In: Paniagua-Zambrana NY, Bussmann RW (Eds.) Ethnobotany of Mountain Regions - Ethnobotany of the Andes. Springer International Publishing, Cham. doi: 10.1007/978-3-319-77093-2_181-1

Paniagua-Zambrana NY, Bussmann RW, Romero C. 2020. Marrubium vulgare L. In: Paniagua-Zambrana NY, Bussmann RW (Eds.) Ethnobotany of Mountain Regions - Ethnobotany of the Andes. Springer International Publishing, Cham. doi: 10.1007/978-3-319-77093-2_182-1

Paniagua-Zambrana NY, Bussmann RW, Romero C. 2020. Mauria heterophylla Kunth. In: Paniagua-Zambrana NY, Bussmann RW (Eds.) Ethnobotany of Mountain Regions - Ethnobotany of the Andes. Springer International Publishing, Cham. doi: 10.1007/978-3-319-77093-2_184-1

Paniagua-Zambrana NY, Bussmann RW, Romero C. 2020. Maytenus laevis Reissek. In: Paniagua-Zambrana NY, Bussmann RW (Eds.) Ethnobotany of Mountain Regions - Ethnobotany of the Andes. Springer International Publishing, Cham. doi: 10.1007/978-3-319-77093-2_185-1

Paniagua-Zambrana NY, Bussmann RW, Romero C. 2020. Medicago sativa L. In: Paniagua-Zambrana NY, Bussmann RW (Eds.) Ethnobotany of Mountain Regions - Ethnobotany of the Andes. Springer International Publishing, Cham. doi: 10.1007/978-3-319-77093-2_186-1

Paniagua-Zambrana NY, Bussmann RW, Romero C. 2020. Melilotus albus Medik., Melilotus officinalis (L.) Lam. In: Paniagua-Zambrana NY, Bussmann RW (Eds.) Ethnobotany of Mountain Regions - Ethnobotany of the Andes. Springer International Publishing, Cham. doi: 10.1007/978-3-319-77093-2_187-1

Paniagua-Zambrana NY, Bussmann RW, Romero C. 2020. Melissa offcinalis L. In: Paniagua-Zambrana NY, Bussmann RW (Eds.) Ethnobotany of Mountain Regions - Ethnobotany of the Andes. Springer International Publishing, Cham. doi: 10.1007/978-3-319-77093-2_188-1

Paniagua-Zambrana NY, Bussmann RW, Romero C. 2020. Minthostachys mollis Grieseb. In: Paniagua-Zambrana NY, Bussmann RW (Eds.) Ethnobotany of Mountain Regions - Ethnobotany of the Andes. Springer International Publishing, Cham. doi: 10.1007/978-3-319-77093-2_191-1

Paniagua-Zambrana NY, Bussmann RW, Romero C. 2020. Momordica charantia L. In: Paniagua-Zambrana NY, Bussmann RW (Eds.) Ethnobotany of Mountain Regions - Ethnobotany of the Andes. Springer International Publishing, Cham. doi: 10.1007/978-3-319-77093-2_192-1

Paniagua-Zambrana NY, Bussmann RW, Romero C. 2020. Myroxylon balsamum (L.) Harms. In: Paniagua-Zambrana NY, Bussmann RW (Eds.) Ethnobotany of Mountain Regions - Ethnobotany of the Andes. Springer International Publishing, Cham. doi: 10.1007/978-3-319-77093-2_291-1

Paniagua-Zambrana NY, Bussmann RW, Romero C. 2020. Nasturtium officinale W.T Aiton. In: Paniagua-Zambrana NY, Bussmann RW (Eds.) Ethnobotany of Mountain Regions - Ethnobotany of the Andes. Springer International Publishing, Cham. doi: 10.1007/978-3-319-77093-2_202-1

Paniagua-Zambrana NY, Bussmann RW, Romero C. 2020. Neurolaena lobata (L.) Cass. In: Paniagua-Zambrana NY, Bussmann RW (Eds.) Ethnobotany of Mountain Regions - Ethnobotany of the Andes. Springer International Publishing, Cham. doi: 10.1007/978-3-319-77093-2_204-1

Paniagua-Zambrana NY, Bussmann RW, Romero C. 2020. Niphidium crassifolium (L.) Lellinger, Polypodium sp. In: Paniagua-Zambrana NY, Bussmann RW (Eds.) Ethnobotany of Mountain Regions - Ethnobotany of the Andes. Springer International Publishing, Cham. doi: 10.1007/978-3-319-77093-2_205-1

Paniagua-Zambrana NY, Bussmann RW, Romero C. 2020. Ocimum basilicum L. In: Paniagua-Zambrana NY, Bussmann RW (Eds.) Ethnobotany of Mountain Regions - Ethnobotany of the Andes. Springer International Publishing, Cham. doi: 10.1007/978-3-319-77093-2_208-1

Paniagua-Zambrana NY, Bussmann RW, Romero C. 2020. Opuntia ficus-indica (L.) Mill. In: Paniagua-Zambrana NY, Bussmann RW (Eds.) Ethnobotany of Mountain Regions - Ethnobotany of the Andes. Springer International Publishing, Cham. doi: 10.1007/978-3-319-77093-2_210-1 
Paniagua-Zambrana NY, Bussmann RW, Romero C. 2020. Origanum majorana L., Origanum vulgare L. In: PaniaguaZambrana NY, Bussmann RW (Eds.) Ethnobotany of Mountain Regions - Ethnobotany of the Andes. Springer International Publishing, Cham. doi: 10.1007/978-3-319-77093-2_213-1

Paniagua-Zambrana NY, Bussmann RW, Romero C. 2020. Oxalis tuberosa Molina. In: Paniagua-Zambrana NY, Bussmann RW (Eds.) Ethnobotany of Mountain Regions - Ethnobotany of the Andes. Springer International Publishing, Cham. doi: 10.1007/978-3-319-77093-2_216-1

Paniagua-Zambrana NY, Bussmann RW, Romero C. 2020. Papaver somniferum L. In: Paniagua-Zambrana NY, Bussmann RW (Eds.) Ethnobotany of Mountain Regions - Ethnobotany of the Andes. Springer International Publishing, Cham. doi: 10.1007/978-3-319-77093-2_218-1

Paniagua-Zambrana NY, Bussmann RW, Romero C. 2020. Passiflora caerulea L., Passiflora edulis L., Passiflora ligularis Juss., Passiflora mollissima (Kunth) L.H. Bailey, Passiflora punctata L., Passiflora quadrangularis L. In: Paniagua-Zambrana NY, Bussmann RW (Eds.) Ethnobotany of Mountain Regions - Ethnobotany of the Andes. Springer International Publishing, Cham. doi: 10.1007/978-3-319-77093-2_221-1

Paniagua-Zambrana NY, Bussmann RW, Romero C. 2020. Pelargonium odoratissimum (L.) L'Hér., Pelargonium roseum Willd., Pelargonium zonale (L.) L'Hér. In: Paniagua-Zambrana NY, Bussmann RW (Eds.) Ethnobotany of Mountain Regions - Ethnobotany of the Andes. Springer International Publishing, Cham. doi: 10.1007/978-3-31977093-2_222-1

Paniagua-Zambrana NY, Bussmann RW, Romero C. 2020. Peperomia alata Ruiz \& Pav., Peperomia blanda (Jacq.) Kunth, Peperomia fraseri C. DC., Peperomia galioides Kunth, Peperomia hartwegiana Miq., Peperomia inaequalifolia Ruz. \& Pav, Pepromia quadrifolia (L.) Kunth. In: Paniagua-Zambrana NY, Bussmann RW (Eds.) Ethnobotany of Mountain Regions - Ethnobotany of the Andes. Springer International Publishing, Cham. doi: 10.1007/978-3-31977093-2_223-1

Paniagua-Zambrana NY, Bussmann RW, Romero C. 2020. Persea americana Mill. In: Paniagua-Zambrana NY, Bussmann RW (Eds.) Ethnobotany of Mountain Regions - Ethnobotany of the Andes. Springer International Publishing, Cham. doi: 10.1007/978-3-319-77093-2_225-1

Paniagua-Zambrana NY, Bussmann RW, Romero C. 2020. Petiveria alliacea L. In: Paniagua-Zambrana NY, Bussmann RW (Eds.) Ethnobotany of Mountain Regions - Ethnobotany of the Andes. Springer International Publishing, Cham. doi: 10.1007/978-3-319-77093-2_26-1

Paniagua-Zambrana NY, Bussmann RW, Romero C. 2020. Petroselinum crispum (Mill.) Fuss. In: Paniagua-Zambrana NY, Bussmann RW (Eds.) Ethnobotany of Mountain Regions - Ethnobotany of the Andes. Springer International Publishing, Cham. doi: 10.1007/978-3-319-77093-2_227-1

Paniagua-Zambrana NY, Bussmann RW, Romero C. 2020. Peumus boldus Molina. In: Paniagua-Zambrana NY, Bussmann RW (Eds.) Ethnobotany of Mountain Regions - Ethnobotany of the Andes. Springer International Publishing, Cham. doi: 10.1007/978-3-319-77093-2_228-1

Paniagua-Zambrana NY, Bussmann RW, Romero C. 2020. Phyllanthus niruri L., Phyllanthus urinaria L., Phyllantus stipulatus (Raf.) G.L. Webster. In: Paniagua-Zambrana NY, Bussmann RW (Eds.) Ethnobotany of Mountain Regions Ethnobotany of the Andes. Springer International Publishing, Cham. doi: 10.1007/978-3-319-77093-2_230-1

Paniagua-Zambrana NY, Bussmann RW, Romero C. 2020. Phytolacca icosandra L., Phytolacca bogotensis Kunth. In: Paniagua-Zambrana NY, Bussmann RW (Eds.) Ethnobotany of Mountain Regions - Ethnobotany of the Andes. Springer International Publishing, Cham. doi: 10.1007/978-3-319-77093-2_231-1

Paniagua-Zambrana NY, Bussmann RW, Romero C. 2020. Pimpinella anisum L. In: Paniagua-Zambrana NY, Bussmann RW (Eds.) Ethnobotany of Mountain Regions - Ethnobotany of the Andes. Springer International Publishing, Cham. doi: 10.1007/978-3-319-77093-2_232-1

Paniagua-Zambrana NY, Bussmann RW, Romero C. 2020. Piper acutifolium Ruiz. \& Pav., Piper aduncum L., Piper aequale Vahl. In: Paniagua-Zambrana NY, Bussmann RW (Eds.) Ethnobotany of Mountain Regions - Ethnobotany of the Andes. Springer International Publishing, Cham. doi: 10.1007/978-3-319-77093-2_233-1

Paniagua-Zambrana NY, Bussmann RW, Romero C. 2020. Porophyllum ruderale (Jacq.) Cass. In: PaniaguaZambrana NY, Bussmann RW (Eds.) Ethnobotany of Mountain Regions - Ethnobotany of the Andes. Springer International Publishing, Cham. doi: 10.1007/978-3-319-77093-2_240-1 
Paniagua-Zambrana NY, Bussmann RW, Romero C. 2020. Portulaca oleracea L. In: Paniagua-Zambrana NY, Bussmann RW (Eds.) Ethnobotany of Mountain Regions - Ethnobotany of the Andes. Springer International Publishing, Cham. doi: 10.1007/978-3-319-77093-2_21-1

Paniagua-Zambrana NY, Bussmann RW, Romero C. 2020. Psidium guajava L., Psidium guineense Sw. In: PaniaguaZambrana NY, Bussmann RW (Eds.) Ethnobotany of Mountain Regions - Ethnobotany of the Andes. Springer International Publishing, Cham. doi: 10.1007/978-3-319-77093-2_245-1

Paniagua-Zambrana NY, Bussmann RW, Romero C. 2020. Pyrethrum parthenium (L.) Sm. In: Paniagua-Zambrana NY, Bussmann RW (Eds.) Ethnobotany of Mountain Regions - Ethnobotany of the Andes. Springer International Publishing, Cham. doi: 10.1007/978-3-319-77093-2_247-1

Paniagua-Zambrana NY, Bussmann RW, Romero C. 2020. Raphanus sativus L. In: Paniagua-Zambrana NY, Bussmann RW (Eds.) Ethnobotany of Mountain Regions - Ethnobotany of the Andes. Springer International Publishing, Cham. doi: 10.1007/978-3-319-77093-2_248-1

Paniagua-Zambrana NY, Bussmann RW, Romero C. 2020. Rheum officinale Baill., Rheum palmatum L. In: PaniaguaZambrana NY, Bussmann RW (Eds.) Ethnobotany of Mountain Regions - Ethnobotany of the Andes. Springer International Publishing, Cham. doi: 10.1007/978-3-319-77093-2_250-1

Paniagua-Zambrana NY, Bussmann RW, Romero C. 2020. Ricinus communis L. In: Paniagua-Zambrana NY, Bussmann RW (Eds.) Ethnobotany of Mountain Regions - Ethnobotany of the Andes. Springer International Publishing, Cham. doi: 10.1007/978-3-319-77093-2_251-1

Paniagua-Zambrana NY, Bussmann RW, Romero C. 2020. Rubus robustus C. Presl. In: Paniagua-Zambrana NY, Bussmann RW (Eds.) Ethnobotany of Mountain Regions - Ethnobotany of the Andes. Springer International Publishing, Cham. doi: 10.1007/978-3-319-77093-2_254-1

Paniagua-Zambrana NY, Bussmann RW, Romero C. 2020. Ruta chalepensis L., Ruta graveolens L. In: PaniaguaZambrana NY, Bussmann RW (Eds.) Ethnobotany of Mountain Regions - Ethnobotany of the Andes. Springer International Publishing, Cham. doi: 10.1007/978-3-319-77093-2_256-1

Paniagua-Zambrana NY, Bussmann RW, Romero C. 2020. Salix chilensis Molina. In: Paniagua-Zambrana NY, Bussmann RW (Eds.) Ethnobotany of Mountain Regions - Ethnobotany of the Andes. Springer International Publishing, Cham. doi: 10.1007/978-3-319-77093-2_257-1

Paniagua-Zambrana NY, Bussmann RW, Romero C. 2020. Salvia ayavacensis Kunth, Salvia cuspidata Ruiz \& Pav., Salvia discolor Kunth, Salvia hispanica L., Salvia macrophylla Benth., Salvia microphylla Kunth, Salvia officinalis L., Salvia palifolia Kunth, Salvia rosmarinifolia G. Don., Salvia sagittata Ruiz \& Pav., Salvia tubiflora Sm. In: PaniaguaZambrana NY, Bussmann RW (Eds.) Ethnobotany of Mountain Regions - Ethnobotany of the Andes. Springer International Publishing, Cham. doi: 10.1007/978-3-319-77093-2_129-1

Paniagua-Zambrana NY, Bussmann RW, Romero C. 2020. Salvia officinalis, Salvia rosmarinus Scheid. In: PaniaguaZambrana NY, Bussmann RW (Eds.) Ethnobotany of Mountain Regions - Ethnobotany of the Andes. Springer International Publishing, Cham. doi: 10.1007/978-3-319-77093-2_308-1

Paniagua-Zambrana NY, Bussmann RW, Romero C. 2020. Sambucus canadensis L., Sambucus nigra L. In: PaniaguaZambrana NY, Bussmann RW (Eds.) Ethnobotany of Mountain Regions - Ethnobotany of the Andes. Springer International Publishing, Cham. doi: 10.1007/978-3-319-77093-2_259-1

Paniagua-Zambrana NY, Bussmann RW, Romero C. 2020. Sanguisorba minor Scop. In: Paniagua-Zambrana NY, Bussmann RW (Eds.) Ethnobotany of Mountain Regions - Ethnobotany of the Andes. Springer International Publishing, Cham. doi: 10.1007/978-3-319-77093-2_260-1

Paniagua-Zambrana NY, Bussmann RW, Romero C. 2020. Scoparia dulcis L. In: Paniagua-Zambrana NY, Bussmann RW (Eds.) Ethnobotany of Mountain Regions - Ethnobotany of the Andes. Springer International Publishing, Cham. doi: 10.1007/978-3-319-77093-2_264-1

Paniagua-Zambrana NY, Bussmann RW, Romero C. 2020. Sida rhombifolia L. In: Paniagua-Zambrana NY, Bussmann RW (Eds.) Ethnobotany of Mountain Regions - Ethnobotany of the Andes. Springer International Publishing, Cham. doi: 10.1007/978-3-319-77093-2_266-1 
Paniagua-Zambrana NY, Bussmann RW, Romero C. 2020. Sigesbeckia orientalis L. In: Paniagua-Zambrana NY, Bussmann RW (Eds.) Ethnobotany of Mountain Regions - Ethnobotany of the Andes. Springer International Publishing, Cham. doi: 10.1007/978-3-319-77093-2_267-1

Paniagua-Zambrana NY, Bussmann RW, Romero C. 2020. Silybum marianum (L.) Gaertn. In: Paniagua-Zambrana NY, Bussmann RW (Eds.) Ethnobotany of Mountain Regions - Ethnobotany of the Andes. Springer International Publishing, Cham. doi: 10.1007/978-3-319-77093-2_268-1

Paniagua-Zambrana NY, Bussmann RW, Romero C. 2020. Smilax kunthii Killip \& C.V. Morton, Smilax medica Schltdkl. \& Cham., Smilax officinalis Kunth. In: Paniagua-Zambrana NY, Bussmann RW (Eds.) Ethnobotany of Mountain Regions - Ethnobotany of the Andes. Springer International Publishing, Cham. doi: 10.1007/978-3-31977093-2_269-1

Paniagua-Zambrana NY, Bussmann RW, Romero C. 2020. Solanum melongena L., Solanum tuberosum L. In: Paniagua-Zambrana NY, Bussmann RW (Eds.) Ethnobotany of Mountain Regions - Ethnobotany of the Andes. Springer International Publishing, Cham. doi: 10.1007/978-3-319-77093-2_271-1

Paniagua-Zambrana NY, Bussmann RW, Romero C. 2020. Sonchus asper (L.) Hill, Sonchus oleraceus L. In: PaniaguaZambrana NY, Bussmann RW (Eds.) Ethnobotany of Mountain Regions - Ethnobotany of the Andes. Springer International Publishing, Cham. doi: 10.1007/978-3-319-77093-2_272-1

Paniagua-Zambrana NY, Bussmann RW, Romero C. 2020. Spartium junceum L. In: Paniagua-Zambrana NY, Bussmann RW (Eds.) Ethnobotany of Mountain Regions - Ethnobotany of the Andes. Springer International Publishing, Cham. doi: 10.1007/978-3-319-77093-2_273-1

Paniagua-Zambrana NY, Bussmann RW, Romero C. 2020. Tamarindus indica L. In: Paniagua-Zambrana NY, Bussmann RW (Eds.) Ethnobotany of Mountain Regions - Ethnobotany of the Andes. Springer International Publishing, Cham. doi: 10.1007/978-3-319-77093-2_277-1

Paniagua-Zambrana NY, Bussmann RW, Romero C. 2020. Taraxacum officinale F.H. Wigg. In: Paniagua-Zambrana NY, Bussmann RW (Eds.) Ethnobotany of Mountain Regions - Ethnobotany of the Andes. Springer International Publishing, Cham. doi: 10.1007/978-3-319-77093-2_280-1

Paniagua-Zambrana NY, Bussmann RW, Romero C. 2020. Theobroma cacao L. In: Paniagua-Zambrana NY, Bussmann RW (Eds.) Ethnobotany of Mountain Regions - Ethnobotany of the Andes. Springer International Publishing, Cham. doi: 10.1007/978-3-319-77093-2_282-1

Paniagua-Zambrana NY, Bussmann RW, Romero C. 2020. Thevetia peruviana (Pers.) K. Schum. In: PaniaguaZambrana NY, Bussmann RW (Eds.) Ethnobotany of Mountain Regions - Ethnobotany of the Andes. Springer International Publishing, Cham. doi: 10.1007/978-3-319-77093-2_284-1

Paniagua-Zambrana NY, Bussmann RW, Romero C. 2020. Thymus vulgaris L. In: Paniagua-Zambrana NY, Bussmann RW (Eds.) Ethnobotany of Mountain Regions - Ethnobotany of the Andes. Springer International Publishing, Cham. doi: 10.1007/978-3-319-77093-2_286-1

Paniagua-Zambrana NY, Bussmann RW, Romero C. 2020. Triticum aestivum L. In: Paniagua-Zambrana NY, Bussmann RW (Eds.) Ethnobotany of Mountain Regions - Ethnobotany of the Andes. Springer International Publishing, Cham. doi: 10.1007/978-3-319-77093-2_290-1

Paniagua-Zambrana NY, Bussmann RW, Romero C. 2020. Uncaria guianensis (Aubl.) J.F. Gmel., Uncaria tomentosa (Willd.) DC., Mimosa acantholoba (Humb. \& Bonpl. ex Willd.) Poir. In: Paniagua-Zambrana NY, Bussmann RW (Eds.) Ethnobotany of Mountain Regions - Ethnobotany of the Andes. Springer International Publishing, Cham. doi: 10.1007/978-3-319-77093-2_292-1

Paniagua-Zambrana NY, Bussmann RW, Romero C. 2020. Vicia faba L. In: Paniagua-Zambrana NY, Bussmann RW (Eds.) Ethnobotany of Mountain Regions - Ethnobotany of the Andes. Springer International Publishing, Cham. doi: 10.1007/978-3-319-77093-2_296-1

Paniagua-Zambrana NY, Bussmann RW, Romero C. 2020. Viola odorata L., Viola tricolor L. In: Paniagua-Zambrana NY, Bussmann RW (Eds.) Ethnobotany of Mountain Regions - Ethnobotany of the Andes. Springer International Publishing, Cham. doi: 10.1007/978-3-319-77093-2_297-1 
Paniagua-Zambrana NY, Bussmann RW, Romero C. 2020. Vitis vinifera L. In: Paniagua-Zambrana NY, Bussmann RW (Eds.) Ethnobotany of Mountain Regions - Ethnobotany of the Andes. Springer International Publishing, Cham. doi: 10.1007/978-3-319-77093-2_298-1

Paniagua-Zambrana NY, Bussmann RW, Romero C. 2020. Xylopia aromatica (Lam.) Mart., Xylopia peruviana R.E. Fr. In: Paniagua-Zambrana NY, Bussmann RW (Eds.) Ethnobotany of Mountain Regions - Ethnobotany of the Andes. Springer International Publishing, Cham. doi: 10.1007/978-3-319-77093-2_301-1

Paniagua-Zambrana NY, Bussmann RW, Romero C. 2020. Zea mays L. In: Paniagua-Zambrana NY, Bussmann RW (Eds.) Ethnobotany of Mountain Regions - Ethnobotany of the Andes. Springer International Publishing, Cham. doi: 10.1007/978-3-319-77093-2_302-1

Paniagua-Zambrana NY, Bussmann RW, Romero C. 2020. Zingiber officinale Roscoe. In: Paniagua-Zambrana NY, Bussmann RW (Eds.) Ethnobotany of Mountain Regions - Ethnobotany of the Andes. Springer International Publishing, Cham. doi: 10.1007/978-3-319-77093-2_303-1

Paniagua-Zambrana NY, Bussmann RW. 2020. Bomarea angustifolia Benth., Bomarea dulcis (Hook.) Beauverd. In: Paniagua-Zambrana NY, Bussmann RW (Eds.) Ethnobotany of Mountain Regions - Ethnobotany of the Andes. Springer International Publishing, Cham. doi: 10.1007/978-3-319-77093-2_306-1

Paniagua-Zambrana NY, Bussmann RW. 2020. Buddleja americana L., Buddleja coriacea J. Rémy. In: PaniaguaZambrana NY, Bussmann RW (Eds.) Ethnobotany of Mountain Regions - Ethnobotany of the Andes. Springer International Publishing, Cham. doi: 10.1007/978-3-319-77093-2_46-1

Paniagua-Zambrana NY, Bussmann RW. 2020. Cantua buxifolia Juss. ex Lam., Cantua flexuosa (Ruiz \& Pav.) Pers., Cantua quercifolia Juss. In: Paniagua-Zambrana NY, Bussmann RW (Eds.) Ethnobotany of Mountain Regions Ethnobotany of the Andes. Springer International Publishing, Cham. doi: 10.1007/978-3-319-77093-2_52-1

Paniagua-Zambrana NY, Bussmann RW. 2020. Capparis crotonoides (Kunth) Iltis \& Cornejo, Capparis scabrida Kunth. In: Paniagua-Zambrana NY, Bussmann RW (Eds.) Ethnobotany of Mountain Regions - Ethnobotany of the Andes. Springer International Publishing, Cham. doi: 10.1007/978-3-319-77093-2_53-1

Paniagua-Zambrana NY, Bussmann RW. 2020. Centaurium erythraea Rafn. In: Paniagua-Zambrana NY, Bussmann RW (Eds.) Ethnobotany of Mountain Regions - Ethnobotany of the Andes. Springer International Publishing, Cham. doi: 10.1007/978-3-319-77093-2_60-1

Paniagua-Zambrana NY, Bussmann RW. 2020. Ceroxylon parvifrons (Engl). H. Wendl., Ceroxylon quindiuense $(\mathrm{H}$. Karst). H. Wendl., Ceroxylon vogelianum (Engl). H. Wendl. In: Paniagua-Zambrana NY, Bussmann RW (Eds.) Ethnobotany of Mountain Regions - Ethnobotany of the Andes. Springer International Publishing, Cham. doi: 10.1007/978-3-319-77093-2_62-1

Paniagua-Zambrana NY, Bussmann RW. 2020. Clusia lechleri Rusby, Clusia minor L., Clusia pachamamae Zenteno Ruiz \& A. Fuentes, Clusia sp., CLUSIACEAE In: Paniagua-Zambrana NY, Bussmann RW (Eds.) Ethnobotany of Mountain Regions - Ethnobotany of the Andes. Springer International Publishing, Cham. doi: 10.1007/978-3-319-77093-2_751

Paniagua-Zambrana NY, Bussmann RW. 2020. Corynaea crassa Hook. f. In: Paniagua-Zambrana NY, Bussmann RW (Eds.) Ethnobotany of Mountain Regions - Ethnobotany of the Andes. Springer International Publishing, Cham. doi: 10.1007/978-3-319-77093-2_82-1

Paniagua-Zambrana NY, Bussmann RW. 2020. Cucumis dipsaceus Ehrenb. ex Spach. In: Paniagua-Zambrana NY, Bussmann RW (Eds.) Ethnobotany of Mountain Regions - Ethnobotany of the Andes. Springer International Publishing, Cham. doi: 10.1007/978-3-319-77093-2_86-1

Paniagua-Zambrana NY, Bussmann RW. 2020. Cuphea carthagenensis (Jacq.) J.F. Macbr., Cuphea ciliata Ruiz \& Pav., Cuphea racemosa (L. f.) Spreng., Cuphea strigulosa Kunth. In: Paniagua-Zambrana NY, Bussmann RW (Eds.) Ethnobotany of Mountain Regions - Ethnobotany of the Andes. Springer International Publishing, Cham. doi: 10.1007/978-3-319-77093-2_90-1

Paniagua-Zambrana NY, Bussmann RW. 2020. Cupressus lusitanica Mill, Cupressa sargentii Jeps. In: PaniaguaZambrana NY, Bussmann RW (Eds.) Ethnobotany of Mountain Regions - Ethnobotany of the Andes. Springer International Publishing, Cham. doi: 10.1007/978-3-319-77093-2_91-1 
Paniagua-Zambrana NY, Bussmann RW. 2020. Descurainia sophia L. In: Paniagua-Zambrana NY, Bussmann RW (Eds.) Ethnobotany of Mountain Regions - Ethnobotany of the Andes. Springer International Publishing, Cham. doi: 10.1007/978-3-319-77093-2_96-1

Paniagua-Zambrana NY, Bussmann RW. 2020. Dictyocaryum lamarckianum (Mart.) H. Wendl. In: PaniaguaZambrana NY, Bussmann RW (Eds.) Ethnobotany of Mountain Regions - Ethnobotany of the Andes. Springer International Publishing, Cham. doi: 10.1007/978-3-319-77093-2_99-1

Paniagua-Zambrana NY, Bussmann RW. 2020. Echinopsis pachanoi (Britton \& Rose) Friedrich \& G.D. In: PaniaguaZambrana NY, Bussmann RW (Eds.) Ethnobotany of Mountain Regions - Ethnobotany of the Andes. Springer International Publishing, Cham. doi: 10.1007/978-3-319-77093-2_107-1

Paniagua-Zambrana NY, Bussmann RW. 2020. Erodium cicutarium (L.) L'Hér. ex Aiton. In: Paniagua-Zambrana NY, Bussmann RW (Eds.) Ethnobotany of Mountain Regions - Ethnobotany of the Andes. Springer International Publishing, Cham. doi: 10.1007/978-3-319-77093-2_113-2

Paniagua-Zambrana NY, Bussmann RW. 2020. Gentianella alborosea (Gilg.) Fabris ex J.S. Pringle, Gentianella bicolor (Wedd.) Fabris ex J.S. Pringle, Gentianella brunneotincta (Gilg) Pringle, Gentianella crassicaulis J.S. Pringle, Gentianella dianthoides (Kunth) Fabris ex J.S. Pringle, Gentianella graminea (Kunth) Fabris, Gentianella thyrsoidea (Hook.) Fabris. In: Paniagua-Zambrana NY, Bussmann RW (Eds.) Ethnobotany of Mountain Regions - Ethnobotany of the Andes. Springer International Publishing, Cham. doi: 10.1007/978-3-319-77093-2_127-1

Paniagua-Zambrana NY, Bussmann RW. 2020. Geranium ayavacense Willd. ex Kunth, Geranium humbloldtii Willd. ex Spreng., Geranium sessiliflorum Cav. In: Paniagua-Zambrana NY, Bussmann RW (Eds.) Ethnobotany of Mountain Regions - Ethnobotany of the Andes. Springer International Publishing, Cham.

Paniagua-Zambrana NY, Bussmann RW. 2020. Hypericum aciculare Kunth, Hypericum laricifolium Juss., Hypericum thesiifolium Kunth. In: Paniagua-Zambrana NY, Bussmann RW (Eds.) Ethnobotany of Mountain Regions Ethnobotany of the Andes. Springer International Publishing, Cham. doi: 10.1007/978-3-319-77093-2_144-1

Paniagua-Zambrana NY, Bussmann RW. 2020. Krameria lappacea (Dombey) Burdet \& B.B. Simpson. In: PaniaguaZambrana NY, Bussmann RW (Eds.) Ethnobotany of Mountain Regions - Ethnobotany of the Andes. Springer International Publishing, Cham. doi: 10.1007/978-3-319-77093-2_158-1

Paniagua-Zambrana NY, Bussmann RW. 2020. Lampayo medicinalis F. Phil. In: Paniagua-Zambrana NY, Bussmann RW (Eds.) Ethnobotany of Mountain Regions - Ethnobotany of the Andes. Springer International Publishing, Cham. doi: 10.1007/978-3-319-77093-2_161-2

Paniagua-Zambrana NY, Bussmann RW. 2020. Lavandula angustifola Mill., Lavandula latifolia Medik. In: PaniaguaZambrana NY, Bussmann RW (Eds.) Ethnobotany of Mountain Regions - Ethnobotany of the Andes. Springer International Publishing, Cham. doi: 10.1007/978-3-319-77093-2_164-1

Paniagua-Zambrana NY, Bussmann RW. 2020. Lepechinia meyenii (Walp.) Epling. In: Paniagua-Zambrana NY, Bussmann RW (Eds.) Ethnobotany of Mountain Regions - Ethnobotany of the Andes. Springer International Publishing, Cham. doi: 10.1007/978-3-319-77093-2_165-1

Paniagua-Zambrana NY, Bussmann RW. 2020. Ligaria cuneifolia (Ruiz \& Pav.) Tiegh. In: Paniagua-Zambrana NY, Bussmann RW (Eds.) Ethnobotany of Mountain Regions - Ethnobotany of the Andes. Springer International Publishing, Cham. doi: 10.1007/978-3-319-77093-2_167-1

Paniagua-Zambrana NY, Bussmann RW. 2020. Loricaria ferruginea (Ruiz \& Pav.) Wedd., Loricaria pauciflora Cuatrec., Loricaria thyrsoidea (Cuatrec.) M.O. Dillon \& Sagást. In: Paniagua-Zambrana NY, Bussmann RW (Eds.) Ethnobotany of Mountain Regions - Ethnobotany of the Andes. Springer International Publishing, Cham. doi: 10.1007/978-3-319-77093-2_175-1

Paniagua-Zambrana NY, Bussmann RW. 2020. Morus alba L. In: Paniagua-Zambrana NY, Bussmann RW (Eds.) Ethnobotany of Mountain Regions - Ethnobotany of the Andes. Springer International Publishing, Cham. doi: 10.1007/978-3-319-77093-2_193-1

Paniagua-Zambrana NY, Bussmann RW. 2020. Muehlenbeckia volcanica (Benth.) Endl. In: Paniagua-Zambrana NY, Bussmann RW (Eds.) Ethnobotany of Mountain Regions - Ethnobotany of the Andes. Springer International Publishing, Cham. doi: 10.1007/978-3-319-77093-2_195-1 
Paniagua-Zambrana NY, Bussmann RW. 2020. Myrcianthes hallii (O. Berg.) McVaugh. Myrcianthes discolor (Kunth) McVaugh Myrcianthes fragrans (Kunth) McVaugh Myrcianthes hallii (O. Berg.) McVaugh Myrtaceae In: PaniaguaZambrana NY, Bussmann RW (Eds.) Ethnobotany of Mountain Regions - Ethnobotany of the Andes. Springer International Publishing, Cham. doi: 10.1007/978-3-319-77093-2_197-1

Paniagua-Zambrana NY, Bussmann RW. 2020. Myristica fragrans Houtt. In: Paniagua-Zambrana NY, Bussmann RW (Eds.) Ethnobotany of Mountain Regions - Ethnobotany of the Andes. Springer International Publishing, Cham. doi: 10.1007/978-3-319-77093-2_199-1

Paniagua-Zambrana NY, Bussmann RW. 2020. Otholobium pubescens (Poir.) J.W. Grimes. In: Paniagua-Zambrana NY, Bussmann RW (Eds.) Ethnobotany of Mountain Regions - Ethnobotany of the Andes. Springer International Publishing, Cham. doi: 10.1007/978-3-319-77093-2_215-1

Paniagua-Zambrana NY, Bussmann RW. 2020. Phoradendron nervosum Oliv. In: Paniagua-Zambrana NY, Bussmann RW (Eds.) Ethnobotany of Mountain Regions - Ethnobotany of the Andes. Springer International Publishing, Cham. doi: 10.1007/978-3-319-77093-2_229-1

Paniagua-Zambrana NY, Bussmann RW. 2020. Pisum sativum L. In: Paniagua-Zambrana NY, Bussmann RW (Eds.) Ethnobotany of Mountain Regions - Ethnobotany of the Andes. Springer International Publishing, Cham. doi: 10.1007/978-3-319-77093-2_234-1

Paniagua-Zambrana NY, Bussmann RW. 2020. Plukenetia huayllabambana Bussamnn, Téllez, Glenn, Plukenetia volubilis L. In: Paniagua-Zambrana NY, Bussmann RW (Eds.) Ethnobotany of Mountain Regions - Ethnobotany of the Andes. Springer International Publishing, Cham. doi: 10.1007/978-3-319-77093-2_238-1

Paniagua-Zambrana NY, Bussmann RW. 2020. Psammisia ulbrichiana Hoerold. In: Paniagua-Zambrana NY, Bussmann RW (Eds.) Ethnobotany of Mountain Regions - Ethnobotany of the Andes. Springer International Publishing, Cham. doi: 10.1007/978-3-319-77093-2_243-1

Paniagua-Zambrana NY, Bussmann RW. 2020. Rosa centifolia L, Rosa odorata (Andrews) Sweet. In: PaniaguaZambrana NY, Bussmann RW (Eds.) Ethnobotany of Mountain Regions - Ethnobotany of the Andes. Springer International Publishing, Cham. doi: 10.1007/978-3-319-77093-2_252-1

Paniagua-Zambrana NY, Bussmann RW. 2020. Satureja boliviana (Benth.) Briq. In: Paniagua-Zambrana NY, Bussmann RW (Eds.) Ethnobotany of Mountain Regions - Ethnobotany of the Andes. Springer International Publishing, Cham. doi: 10.1007/978-3-319-77093-2_261-1

Paniagua-Zambrana NY, Bussmann RW. 2020. Stachys pusilla (Wedd.) Briq. In: Paniagua-Zambrana NY, Bussmann RW (Eds.) Ethnobotany of Mountain Regions - Ethnobotany of the Andes. Springer International Publishing, Cham. doi: 10.1007/978-3-319-77093-2_274-1

Paniagua-Zambrana NY, Bussmann RW. 2020. Ullucus tuberosus Caldas. In: Paniagua-Zambrana NY, Bussmann RW (Eds.) Ethnobotany of Mountain Regions - Ethnobotany of the Andes. Springer International Publishing, Cham. doi: 10.1007/978-3-319-77093-2_307-1

Paniagua-Zambrana NY. 1999. Estudio comparativo de la densidad y estructura poblacional en poblaciones naturales de Attalea phalerata (Palmae) sometidas a diferente intensidad de extracción en la región noreste de Bolivia. In: Memorias del IV Congreso Latinoamericano de Ecología. Universidad de San Agustín. Arequipa, Perú.

Paniagua-Zambrana NY. 1999. Flora y Vegetación. In: Moraes, M. (Ed.) Estudio de Biodiversidad de la Reserva Nacional de Flora y Fauna Tariquía, Depto.Tarija, Bolivia. Fundación para el Desarrollo de la Ecología (FUNDECO)Instituto de Ecología. La Paz, Bolivia.

Paniagua Zambrana, N, Cámara-Leret R, Macía, MJ. 2014. Patrones de uso de las palmeras en bosques amazónicos y montanos de Bolivia: Género, edad, grado de educación e idioma. In: M. Moraes, editores. Palmeras útiles de Bolivia - Las especies mayormente aprovechadas para diferentes fines y aplicaciones. Herbario Nacional de Bolivia, Universidad Mayor de San Andrés, Plural editores, La Paz. pp. 127-132.

Panta K, Sher H, Jan HA, Ur-Rahman, I, Hussain W, Abbasi AM, Kunwar RM, Bussmann RW, Paniagua-Zambrana NY. 2021. Arisaema flavum (Forssk.) Schott In: Kunwar RW, Sher H, Bussmann RW. Ethnobotany of the Himalayas. Springer, Cham. doi: 10.1007/978-3-030-57408-6_26, pp. 257-266. 
Parajuli R, Paudel A, Jan HA, Hussain W, Abbasi AM, Kunwar RM, Bussmann RW, Paniagua-Zambrana NY. 2021. Rosa brunonii Lindl., Rosa macrophylla Lindl., Rosa sericea Lindl., Rosa webbiana Wall. ex Royle In: Kunwar RW, Sher H, Bussmann RW. Ethnobotany of the Himalayas. Springer, Cham. doi: 10.1007/978-3-030-57408-6_206, pp. 16971708.

Parajuli R, Timilsina B, Jan HA, Sher H, Ur Rahman I, Hussain W, Kunwar RM, Bussmann RW, Paniagua-Zambrana NY. 2021. Delphinium brunonianum Royle, Delphinium himalayae Munz, Delphinium roylei Munz, Delphinium vestitum Wall. ex Royle In: Kunwar RW, Sher H, Bussmann RW. Ethnobotany of the Himalayas. Springer, Cham. doi: 10.1007/978-3-030-57408-6_79, pp. 739-746.

Pariyar S, Paudel HR, Kunwar RM, Bussmann RW, Paniagua-Zambrana NY. 2021. Potentilla fulgens Wall. ex Hook. In: Kunwar RW, Sher H, Bussmann RW. Ethnobotany of the Himalayas. Springer, Cham. doi: 10.1007/978-3-03057408-6_195, pp. 1579-1588.

Paudel A, Parajuli R, Upadhaya S, Kunwar RM, Bussmann RW, Paniagua-Zambrana NY. 2021. Parnassia nubicola Wall. ex Royle. In: Kunwar RW, Sher H, Bussmann RW. Ethnobotany of the Himalayas. Springer, Cham. doi: 10.1007/978-3-030-57408-6_175, pp. 1449-1459.

Paudel HR, Poudel P, Kunwar RM, Sher H, Ur Rahman I, Abbasi AM, Bussmann RW, Paniagua-Zambrana NY. 2021. Aesculus indica (Wall. ex Cambess.) Hook. In: Kunwar RW, Sher H, Bussmann RW. Ethnobotany of the Himalayas. Springer, Cham. doi: 10.1007/978-3-030-57408-6_13, pp. 163-170.

Paudel KC, Kunwar RM, Bussmann RW, Paniagua-Zambrana NY. 2021. Choerospondias axillaris (Roxb.) B. Burtt \& A.W. Hill In: Kunwar RW, Sher H, Bussmann RW. Ethnobotany of the Himalayas. Springer, Cham. doi: 10.1007/978-3030-57408-6_55, pp. 527-540.

Paudel RC, Kunwar RM, Jan HA, Abbasi AM, Bussmann RW, Paniagua-Zambrana NY. 2021. Mallotus phillippinensis (Lam.) Müll. Arg In: Kunwar RW, Sher H, Bussmann RW. Ethnobotany of the Himalayas. Springer, Cham. doi: 10.1007/978-3-030-57408-6_147, pp. 1231-1238.

Pokharel YR, Kunwar RM, Bussmann RW, Paniagua-Zambrana NY, Abbasi, AM. 2021. Hippophae rhamnoides L. ssp. turkestanica Rousi, Hippophae rhamnoides L., Hippophae salicifolia D. Don, Hippophae tibetana Schltdl. In: Kunwar RW, Sher H, Bussmann RW. Ethnobotany of the Himalayas. Springer, Cham. doi: 10.1007/978-3-030-57408-6_117, pp. 1033-1042.

Poude, HR, Kunwar RM, Bussmann RW, Paniagua-Zambrana NY. 2021. Fritillaria cirrhosa D. Don In: Kunwar RW, Sher H, Bussmann RW. Ethnobotany of the Himalayas. Springer, Cham. doi: 10.1007/978-3-030-57408-6_105, pp. 957-964.

Poudel P, Thapa R, Serchan J, Sapkota S, Bussmann RW, Paniagua-Zambrana NY 2021. Mussaenda frondosa L., Mussaenda macrophylla Wall. In: Kunwar RW, Sher H, Bussmann RW. Ethnobotany of the Himalayas. Springer, Cham. doi: 10.1007/978-3-030-57408-6_156, pp. 1327-1336.

Poudel RC, Kunwar RM, Bussmann RW, Paniagua-Zambrana NY. 2021. Astilbe rivularis Buch.-Ham. ex D. Don. In: Kunwar RW, Sher H, Bussmann RW. Ethnobotany of the Himalayas. Springer, Cham. doi: 10.1007/978-3-030-574086_30, pp. 305-310.

Poudel RC, Kunwar RM, Jan HA, Sher H, Ur Rahman I, Hussain W, Bussmann RW, Paniagua-Zambrana NY. 2021. Rhododendron afghanicum Aitch. \& Hemsl., Rhododendron anthopogon D. Don, Rhododendron arboreum Sm., Rhododendron campanulatum D. Don, Rhododendron collettianum Aitch. \& Hemsl. In: Kunwar RW, Sher H, Bussmann RW. Ethnobotany of the Himalayas. Springer, Cham. doi: 10.1007/978-3-030-57408-6_204, pp. $1673-1690$.

Poudel RC, Kunwar RM, Sher H, Ur Rahman I, Bussmann RW, Paniagua-Zambrana NY. 2021. Taxus baccata (L.) Borkh., Taxus contorta Griff., Taxus mairei (Lemée \& H. Lév.) S.Y. Hu, Taxus wallichiana Zucc. In: Kunwar RW, Sher H, Bussmann RW. Ethnobotany of the Himalayas. Springer, Cham. doi: 10.1007/978-3-030-57408-6_243, pp. 1991-2004.

Poudeyal MR, Kunwar RM, Bussmann RW, Paniagua-Zambrana NY. 2021. Meconopsis aculeatal. Royle, Meconopsis horridula Hook. f. and Thomson, Meconopsis latifolia (Prain) Prain, Meconopsis quintuplinervia Regel, Meconopsis simplicifolia (D. Don) Walpers 2021. In: Kunwar RW, Sher H, Bussmann RW. Ethnobotany of the Himalayas. Springer, Cham. doi: 10.1007/978-3-030-57408-6_149, pp. 1247-1256. 
Poudeyal MR, Paudel B, Bhattarai HD, Kunwar RM, Jan HA, Ali M, Bussmann RW, Paniagua-Zambrana NY. 2021. Euphorbia hirta L., Euphorbia pilosa L., Euphorbia royleana Boiss. In: Kunwar RW, Sher H, Bussmann RW. Ethnobotany of the Himalayas. Springer, Cham. doi: 10.1007/978-3-030-57408-6_97, pp. 903-916.

Poudyal BH, Aryal S, Kunwar RM, Jan HA, Abbasi AM, Bussmann RW, Paniagua-Zambrana NY. 2021. Phyllanthus emblica L. In: Kunwar RW, Sher H, Bussmann RW. Ethnobotany of the Himalayas. Springer, Cham. doi: 10.1007/9783-030-57408-6_183, pp. 1495-1504.

Pradhan DK, Ulak S, Charmakar S, Kunwar RM, Bussmann RW, Paniagua-Zambrana NY. 2021. Rubia manjith Roxb. ex Fleming, Rubia tinctorium L., In: Kunwar RW, Sher H, Bussmann RW. Ethnobotany of the Himalayas. Springer, Cham. doi: 10.1007/978-3-030-57408-6_207, pp. 1709-1716.

Prasad Pokhrel N, Prasad Pandey H, Kunwar RM, Bussmann RW, Paniagua-Zambrana NY. 2021. Castanopsis hystrix J. D. Hooker \& Thomson ex A. de Candolle, Castanopsis indica (Roxburgh ex Lindley) A. de Candolle in Hance, Castanopsis tribuloides (Smith) A. de Candolle in Hance. In: Kunwar RW, Sher H, Bussmann RW. Ethnobotany of the Himalayas. Springer, Cham. doi: 10.1007/978-3-030-57408-6_50, pp. 491-496

Pyakurel D, Jan HA, Kunwar RM, Bussmann RW, Paniagua-Zambrana NY. 2021. Cinnamomum tamala (Buch.-Ham.) T. Nees \& Nees In: Kunwar RW, Sher H, Bussmann RW. Ethnobotany of the Himalayas. Springer, Cham. doi: 10.1007/978-3-030-57408-6_57, pp. 547-554.

Ranjitkar S, Sujakhu NM, Kunwar RM, Sher H, Ur Rahman I, Hussain W, Bussmann RW, Paniagua-Zambrana NY. 2021. Morchella conica Pers. ex Fr., Morchella esculenta (Fr.) I.R. Hazll In: Kunwar RW, Sher H, Bussmann RW. Ethnobotany of the Himalayas. Springer, Cham. doi: 10.1007/978-3-030-57408-6_152, pp. 1279-1294.

Romero C, Paniagua-Zambrana NY, Bussmann RW. 2020. Ethnobotany of Mountain Regions - Ethnobotany of the Andes - Colombia and Ecuador. In: Paniagua-Zambrana NY, Bussmann RW (Eds.) Ethnobotany of Mountain Regions - Ethnobotany of the Andes. Springer International Publishing, Cham. doi: 10.1007/978-3-319-77093-2_3-1

Saiju HK, Kunwar RM, Bussmann RW, Paniagua-Zambrana NY. 2021. Luculia gratissima (Wall.) Sweet In: Kunwar RW, Sher H, Bussmann RW. Ethnobotany of the Himalayas. Springer, Cham. doi: 10.1007/978-3-030-57408-6_144, pp. 1211-1214.

Sapkota B, Poudel P, Thapa R, Sherchan J, Kunwar RM, Jan HA, Abbasi AM, Bussmann RW, Paniagua-Zambrana NY. 2021. Zanthoxylum armatum DC. Zanthoxylum oxyphyllum Edgew. In: Kunwar RW, Sher H, Bussmann RW. Ethnobotany of the Himalayas. Springer, Cham. doi: 10.1007/978-3-030-57408-6_261, pp. 2159-2171.

Sapkota P, Poudel RC, Kunwar RM, Bussmann RW, Paniagua-Zambrana NY. 2021. Girardinia diversifolia (Link) Friis In: Kunwar RW, Sher H, Bussmann RW. Ethnobotany of the Himalayas. Springer, Cham. doi: 10.1007/978-3-03057408-6_111, pp. 995-1000.

Sapkota PP, Kunwar RM, Jan HA, Bussmann RW, Paniagua-Zambrana NY. 2021. Coccinia grandis (L.) Voigt In: Kunwar RW, Sher H, Bussmann RW. Ethnobotany of the Himalayas. Springer, Cham. doi: 10.1007/978-3-030-574086_60, pp. 583-590.

Shankar Bhandari G, Kunwar RM, Bussmann RW, Paniagua-Zambrana NY. 2021. Drosera peltata Thuinb. In: Kunwar RW, Sher H, Bussmann RW. Ethnobotany of the Himalayas. Springer, Cham. doi: 10.1007/978-3-030-57408-6_85, pp. 789-794.

Sharma G, Thapa R, Lamichhane S, Poudel HR, Devkota HP, Poudel P, Kunwar RM, Jan HA, Sher H, Ur Rahman I, Abbasi AM, Bussmann RW, Paniagua-Zambrana NY. 2021. Rumex nepalensis Spreng., Rumex hastatus D. Don, Rumex longifolius DC. In: Kunwar RW, Sher H, Bussmann RW. Ethnobotany of the Himalayas. Springer, Cham. doi: 10.1007/978-3-030-57408-6_209, pp. 1735-1754.

Sher H, Bussmann RW, Paniagua-Zambrana NY, Ali, M. 2021. Prunella vulgaris L. In: Kunwar RW, Sher H, Bussmann RW. Ethnobotany of the Himalayas. Springer, Cham. doi: 10.1007/978-3-030-57408-6_198, pp. 1607-1612.

Sher H, Bussmann RW, Paniagua-Zambrana NY, Hussain, W. 2021. Periploca aphylla Decne. In: Kunwar RW, Sher H, Bussmann RW. Ethnobotany of the Himalayas. Springer, Cham. doi: 10.1007/978-3-030-57408-6_178, pp. 14711474. 
Sher H, Bussmann RW, Paniagua-Zambrana NY, Hussain, W. 2021. Phlomis bracteosa Royle ex Benth. In: Kunwar RW, Sher H, Bussmann RW. Ethnobotany of the Himalayas. Springer, Cham. doi: 10.1007/978-3-030-57408-6_181, pp. 1491-1494.

Sher H, Bussmann RW, Paniagua-Zambrana NY, Ur Rahman I, Hussain, W. 2021. Ipomoea hederaceae (L.) Jacq. In: Kunwar RW, Sher H, Bussmann RW. Ethnobotany of the Himalayas. Springer, Cham. doi: 10.1007/978-3-030-574086_126, pp. 1091-1092.

Sher H, Bussmann RW, Paniagua-Zambrana NY, Ur Rahman I, Jan, HA. 2021. Corydalis govaniana Wall. In: Kunwar RW, Sher H, Bussmann RW. Ethnobotany of the Himalayas. Springer, Cham. doi: 10.1007/978-3-030-57408-6_65, pp. 621-626.

Sher H, Bussmann RW, Paniagua-Zambrana NY, Ur Rahman, I. 2021. Cotoneaster uniflora Bunge In: Kunwar RW, Sher H, Bussmann RW. Ethnobotany of the Himalayas. Springer, Cham. doi: 10.1007/978-3-030-57408-6_66, pp. 627628.

Sher H, Bussmann RW, Paniagua-Zambrana NY, Ur Rahman, I. 2021. Digitalis lanata Ehrh. In: Kunwar RW, Sher H, Bussmann RW. Ethnobotany of the Himalayas. Springer, Cham. doi: 10.1007/978-3-030-57408-6_982, pp. 761-768.

Sher H, Bussmann RW, Paniagua-Zambrana NY, Ur Rahman, I. 2021. Fagonia bruguieri DC. var. bruguieri, Fagonia indica var. schweinfurthii Hadidi In: Kunwar RW, Sher H, Bussmann RW. Ethnobotany of the Himalayas. Springer, Cham. doi: 10.1007/978-3-030-57408-6_101, pp. 929-932.

Sher H, Bussmann RW, Paniagua-Zambrana NY, Ur Rahman, I. 2021. Hippolytia dolichophylla (Kitam.) Bremer \& Humphries In: Kunwar RW, Sher H, Bussmann RW. Ethnobotany of the Himalayas. Springer, Cham. doi: 10.1007/9783-030-57408-6_116, pp. 1031-1032.

Sher H, Bussmann RW, Paniagua-Zambrana NY, Ur Rahman, I. 2021. Orobanche vulgaris Poir. In: Kunwar RW, Sher H, Bussmann RW. Ethnobotany of the Himalayas. Springer, Cham. doi: 10.1007/978-3-030-57408-6_167, pp. 13971400 .

Sher H, Bussmann RW, Paniagua-Zambrana NY, Ur Rahman, I. 2021. Parietaria judaica L. In: Kunwar RW, Sher H, Bussmann RW. Ethnobotany of the Himalayas. Springer, Cham. doi: 10.1007/978-3-030-57408-6_173, pp. 1439-1440.

Sher H, Bussmann RW, Paniagua-Zambrana NY, Ur Rahman, I. 2021. Pedicularis albida Penn., Pedicularis tenuirostris Benth. In: Kunwar RW, Sher H, Bussmann RW. Ethnobotany of the Himalayas. Springer, Cham. doi: 10.1007/978-3-030-57408-6_176, pp. 1455-1460.

Sher H, Bussmann RW, Paniagua-Zambrana NY, Ur Rahman, I. 2021. Perovskia abrotanoides Karel. In: Kunwar RW, Sher H, Bussmann RW. Ethnobotany of the Himalayas. Springer, Cham. doi: 10.1007/978-3-030-57408-6_179, pp. 1475-1476.

Sher H, Bussmann RW, Paniagua-Zambrana NY, Ur Rahman, I. 2021. Physochlaina praealta Hook. In: Kunwar RW, Sher H, Bussmann RW. Ethnobotany of the Himalayas. Springer, Cham. doi: 10.1007/978-3-030-57408-6_182, pp. 1505-1506.

Sher H, Bussmann RW, Paniagua-Zambrana NY, Ur Rahman, I. 2021. Picrorhiza kurroa Royle ex Benth. In: Kunwar RW, Sher H, Bussmann RW. Ethnobotany of the Himalayas. Springer, Cham. doi: 10.1007/978-3-030-57408-6_185, pp. 1515-1518.

Sher H, Bussmann RW, Paniagua-Zambrana NY, Ur Rahman, I. 2021. Tamaricaria elegans (Royle) Qaiser \& Ali In: Kunwar RW, Sher H, Bussmann RW. Ethnobotany of the Himalayas. Springer, Cham. doi: 10.1007/978-3-030-574086_240, pp. 1967-1970.

Sher H, Bussmann RW, Paniagua-Zambrana NY, Ur-Rahman, I. 2021. Aconogonon coriarium (Grig.) Sojak., Aconogonon tortuosum (D. Don) Hara var. tibetanum (Meisn.) S.-P.Hong In: Kunwar RW, Sher H, Bussmann RW. Ethnobotany of the Himalayas. Springer, Cham. doi: 10.1007/978-3-030-57408-6_11, pp. 155-160.

Sher H, Bussmann RW, Paniagua-Zambrana NY, Ur-Rahman, I. 2021. Aegopodium alpestre Ledeb. In: Kunwar RW, Sher H, Bussmann RW. Ethnobotany of the Himalayas. Springer, Cham. doi: 10.1007/978-3-030-57408-6_12, pp. 161162. 
Sher H, Bussmann RW, Paniagua-Zambrana NY, Ur-Rahman, I. 2021. Ampelocissus latifolia (Roxb.) Planch. In: Kunwar RW, Sher H, Bussmann RW. Ethnobotany of the Himalayas. Springer, Cham. doi: 10.1007/978-3-030-574086_18, pp. 217-222.

Sher H, Bussmann RW, Paniagua-Zambrana NY, Ur-Rahman, I. 2021. Anacyclus pyrethrum DC In: Kunwar RW, Sher H, Bussmann RW. Ethnobotany of the Himalayas. Springer, Cham. doi: 10.1007/978-3-030-57408-6_20, pp. 223-224.

Sher H, Bussmann RW, Paniagua-Zambrana NY, Ur-Rahman, I. 2021. Carex canescens L., Carex cardiolepis Nees, Carex cruenta Nees In: Kunwar RW, Sher H, Bussmann RW. Ethnobotany of the Himalayas. Springer, Cham. doi: 10.1007/978-3-030-57408-6_47, pp. 461-468

Sher H, Bussmann RW, Paniagua-Zambrana NY. 2021. Bupleurum thomsonii C.B. Clarke In: Kunwar RW, Sher H, Bussmann RW. Ethnobotany of the Himalayas. Springer, Cham. doi: 10.1007/978-3-030-57408-6_41, pp. 423-426.

Sher H, Bussmann RW, Paniagua-Zambrana NY. 2021. Cordia dichotoma G. Forst. In: Kunwar RW, Sher H, Bussmann RW. Ethnobotany of the Himalayas. Springer, Cham. doi: 10.1007/978-3-030-57408-6_64, pp. 615-620.

Sher H, Bussmann RW, Paniagua-Zambrana NY. 2021. Fraxinus hookeri Wenzig, Fraxinus xanthoxyloides (G. Don) DC. In: Kunwar RW, Sher H, Bussmann RW. Ethnobotany of the Himalayas. Springer, Cham. doi: 10.1007/978-3-03057408-6_104, pp. 951-956.

Sher H, Bussmann RW, Paniagua-Zambrana NY. 2021. Trigonella corniculata L., Trigonella gracilis Benth. In: Kunwar RW, Sher H, Bussmann RW. Ethnobotany of the Himalayas. Springer, Cham. doi: 10.1007/978-3-030-57408-6_250, pp. 2055-2060.

Sher H, Bussmann RW, Paniagua-Zambrana NY. 2021. Vernonia anthelmintica (L.) Willd. In: Kunwar RW, Sher H, Bussmann RW. Ethnobotany of the Himalayas. Springer, Cham. doi: 10.1007/978-3-030-57408-6_265, pp. 2113-2122.

Sher H, Bussmann, RTW, Paniagua-Zambrana NY. 2021. Cyperus difformis/ L., Cyperus rotundus L. In: Kunwar RW, Sher H, Bussmann RW. Ethnobotany of the Himalayas. Springer, Cham. doi: 10.1007/978-3-030-57408-6_74, pp. 687694.

Sher H, Hussain W, Kunwar RM, Bussmann RW, Paniagua-Zambrana NY. 2021. Ixiolirion tataricum (Pall.) Herb. In: Kunwar RW, Sher H, Bussmann RW. Ethnobotany of the Himalayas. Springer, Cham. doi: 10.1007/978-3-030-574086_128, pp. 1101-1102.

Sher H, Jan HA, Abbasi AM, Ur Rahman I, Bussmann RW, Paniagua-Zambrana NY. 2021. Pistacia atlantica Desf., Pistacia integerrima Stewart ex Brandis, Pistacia khinjuk Stocks In: Kunwar RW, Sher H, Bussmann RW. Ethnobotany of the Himalayas. Springer, Cham. doi: 10.1007/978-3-030-57408-6_187, pp. 1531-1538.

Sher H, Jan HA, Abbasi AM, Ur Rahman I, Hussain W, Bussmann RW, Paniagua-Zambrana NY. 2021. Colchicum aitchnsonii Hook. f.) Nasir, Colchicum luteum Baker In: Kunwar RW, Sher H, Bussmann RW. Ethnobotany of the Himalayas. Springer, Cham. doi: 10.1007/978-3-030-57408-6_63, pp. 609-614.

Sher H, Jan HA, Ur Rahman I, Hussain W, Kunwar RM, Bussmann RW, Paniagua-Zambrana NY. 2021. Aconitum atrox Walp., Aconitum ferox Wall. ex. Ser., Aconitum balfourii Stapf., Aconitum heterophyllum Wall. ex Royle, Aconitum laeve Royle, Aconitum spicatum Stapf, Aconitum violaceum Jacq. ex Stapf var. violaceum In: Kunwar RW, Sher H, Bussmann RW. Ethnobotany of the Himalayas. Springer, Cham. doi: 10.1007/978-3-030-57408-6_10, pp. 139154.

Sher H, Kunwar RM, Bussmann RW, Paniagua-Zambrana NY, Ur Rahman I, Hussain, W. 2021. Salvia nubicola Wall. ex Sweet In: Kunwar RW, Sher H, Bussmann RW. Ethnobotany of the Himalayas. Springer, Cham. doi: 10.1007/9783-030-57408-6_213, pp. 1763-1774.

Sher H, Kunwar RM, Bussmann RW, Paniagua-Zambrana NY, Ur Rahman, I. 2021. Gentianodes tianschanica (Rupr. ex Kusn.) Omer, Ali \& Qaiser In: Kunwar RW, Sher H, Bussmann RW. Ethnobotany of the Himalayas. Springer, Cham. doi: 10.1007/978-3-030-57408-6_108, pp. 977-980.

Sher H, Kunwar RM, Bussmann RW, Paniagua-Zambrana NY, Ur Rahman, I. 2021. Pleurospermum candollei (DC.) C.B. Clarke In: Kunwar RW, Sher H, Bussmann RW. Ethnobotany of the Himalayas. Springer, Cham. doi: 10.1007/9783-030-57408-6_191, pp. 1559-1560. 
Sher H, Kunwar RM, Bussmann RW, Paniagua-Zambrana NY, Ur Rahman, I. 2021. Salix alba L. In: Kunwar RW, Sher H, Bussmann RW. Ethnobotany of the Himalayas. Springer, Cham. doi: 10.1007/978-3-030-57408-6_211, pp. 17571762.

Sher H, Kunwar RM, Bussmann RW, Paniagua-Zambrana NY. 2021. Erigeron alpinus L. In: Kunwar RW, Sher H, Bussmann RW. Ethnobotany of the Himalayas. Springer, Cham. doi: 10.1007/978-3-030-57408-6_95, pp. 891-896.

Sher H, Kunwar RM, Bussmann RW, Paniagua-Zambrana NY. 2021. Ferula assa-foetida L., Ferula narthex Boiss. In: Kunwar RW, Sher H, Bussmann RW. Ethnobotany of the Himalayas. Springer, Cham. doi: 10.1007/978-3-030-574086_102, pp. 933-940.

Sher H, Kunwar RM, Bussmann RW, Paniagua-Zambrana NY. 2021. Gentianopsis paludosa (Munro ex Hook.f.) Ma, Gentianopsis vvedenskyi (Grossh.) V.V. Pis. In: Kunwar RW, Sher H, Bussmann RW. Ethnobotany of the Himalayas. Springer, Cham. doi: 10.1007/978-3-030-57408-6_109, pp. 981-984.

Sher H, Kunwar RM, Bussmann RW, Paniagua-Zambrana NY. 2021. Heracleum candicans Wall. ex DC. In: Kunwar RW, Sher H, Bussmann RW. Ethnobotany of the Himalayas. Springer, Cham. doi: 10.1007/978-3-030-57408-6_115, pp. 1021-1030.

Sher H, Kunwar RM, Bussmann RW, Paniagua-Zambrana NY. 2021. Isatis stocksii Boiss. In: Kunwar RW, Sher H, Bussmann RW. Ethnobotany of the Himalayas. Springer, Cham. doi: 10.1007/978-3-030-57408-6_127, pp. $1093-1100$.

Sher H, Kunwar RM, Bussmann RW, Paniagua-Zambrana NY. 2021. Juncus articulatus L. In: Kunwar RW, Sher H, Bussmann RW. Ethnobotany of the Himalayas. Springer, Cham. doi: 10.1007/978-3-030-57408-6_133, pp. 1141-1142.

Sher H, Ur Rahman I, Ali M, Bussmann RW, Paniagua-Zambrana NY. 2021. Thymus linearis Benth. ssp. linearis, Thymus serpyllum L., Thymus vulgaris L. In: Kunwar RW, Sher H, Bussmann RW. Ethnobotany of the Himalayas. Springer, Cham. doi: 10.1007/978-3-030-57408-6_246, pp. 2027-2036.

Sher H, Ur Rahman I, Bussmann RW, Paniagua-Zambrana NY. 2021. Paeonia emodi Wall. ex Royle In: Kunwar RW, Sher H, Bussmann RW. Ethnobotany of the Himalayas. Springer, Cham. doi: 10.1007/978-3-030-57408-6_171, pp. 1423-1430.

Sher H, Ur Rahman I, Hussain W, Abbasi AM, Kunwar RM, Bussmann RW, Paniagua-Zambrana NY. 2021. Berberis aristata DC., Berberis asiatica Roxb. ex DC., Berberis chitria Buch.-Ham. ex Ker Gawl., Berberis glaucocarpa Stapf, Berberis lycium Royle, Berberis orthobotrys Bien. ex Aitch. ssp. orthobotrys, Berberis vulgaris L. In: Kunwar RW, Sher H, Bussmann RW. Ethnobotany of the Himalayas. Springer, Cham. doi: 10.1007/978-3-030-57408-6_35, pp. $337-352$.

Sher H, Ur Rahman I, Jan HA, Abbasi AM, Bussmann RW, Paniagua-Zambrana NY. 2021. Bistorta amplexicaulis (D. Don) Greene, Bistorta vivipara (L.) S.F. Gray In: Kunwar RW, Sher H, Bussmann RW. Ethnobotany of the Himalayas. Springer, Cham. doi: 10.1007/978-3-030-57408-6_39, pp. 391-402.

Sher H, Ur Rahman I, Jan HA, Abbasi AM, Bussmann RW, Paniagua-Zambrana NY. 2021. Verbascum thapsus L. In: Kunwar RW, Sher H, Bussmann RW. Ethnobotany of the Himalayas. Springer, Cham. doi: 10.1007/978-3-030-574086_254, pp. 2101-2112.

Sher H, Ur Rahman I, Jan HA, Abbasi AM, Bussmann RW, Paniagua-Zambrana NY. 2021. Viola biflora L., Viola canescens Wall., Viola odorata L., Viola pilosa Blumne, Viola rupestris F.W. Schmidt, Viola suavis M. Bieb. In: Kunwar RW, Sher H, Bussmann RW. Ethnobotany of the Himalayas. Springer, Cham. doi: 10.1007/978-3-030-57408-6_258, pp. 2123-2138.

Sher, H., Bussmann RW, Paniagua-Zambrana NY. 2021. Atriplex hortensis L., Atriplex rosea L. In: Kunwar RW, Sher H, Bussmann RW. Ethnobotany of the Himalayas. Springer, Cham. doi: 10.1007/978-3-030-57408-6_32, pp. 317-322.

Sherchan J, Kunwar RM, Jan HA, Bussmann RW, Paniagua-Zambrana NY. 2021. Eclipta prostrata (L.) L. In: Kunwar RW, Sher H, Bussmann RW. Ethnobotany of the Himalayas. Springer, Cham. doi: 10.1007/978-3-030-57408-6_89, pp. 835-854.

Shrestha HS, Kunwar RM, Ali M. Bussmann RW, Paniagua-Zambrana NY. 2021. Equisetum diffusum D. Don, Equisetum ramosissium Desf. In: Kunwar RW, Sher H, Bussmann RW. Ethnobotany of the Himalayas. Springer, Cham. doi: 10.1007/978-3-030-57408-6_94, pp. 879-890. 
Shrestha HS, Kunwar RM, Bussmann RW, Paniagua-Zambrana NY. 2021. Dryopteris cochleata (Buch.-Ham. ex D. Don) C. Chr., Dryopteris filix-mas (L.) Schott In: Kunwar RW, Sher H, Bussmann RW. Ethnobotany of the Himalayas. Springer, Cham. doi: 10.1007/978-3-030-57408-6_87, pp. 811-820.

Shrestha I, Kunwar RM, Hussain W, Jan HA, Abbasi AM, Bussmann RW, Paniagua-Zambrana NY. 2021. Juniperus communis L., Juniperus excelsa M. Bieb., Juniperus indica Bertol., Juniperus pseudosabina var. turkestanica (Kom.) Silba, Juniperus recurva Buch.-Ham. ex D. Don, Juniperus squamata Lamb., In: Kunwar RW, Sher H, Bussmann RW. Ethnobotany of the Himalayas. Springer, Cham. doi: 10.1007/978-3-030-57408-6_134, pp. 1143-1156.

Shrestha K, Kunwar RM, Bussmann RW, Paniagua-Zambrana NY. 2021. Oroxylum indicum (L.) Kurz In: Kunwar RW, Sher H, Bussmann RW. Ethnobotany of the Himalayas. Springer, Cham. doi: 10.1007/978-3-030-57408-6_168, pp. 1401-1408.

Shrestha K, Singh Saud D, Pant S, Kunwar RM, Jan HA, Bussmann RW, Paniagua-Zambrana NY. 2021. Mucuna pruriens (L.) DC. In: Kunwar RW, Sher H, Bussmann RW. Ethnobotany of the Himalayas. Springer, Cham. doi: 10.1007/978-3-030-57408-6_154, pp. 1309-1316.

Shrestha R, Acharya RP, Bussmann RW, Paniagua-Zambrana NY. 2021. Terminalia belirica (Gaertn.) Roxb., Terminalia chebula Retz. In: Kunwar RW, Sher H, Bussmann RW. Ethnobotany of the Himalayas. Springer, Cham. doi: 10.1007/978-3-030-57408-6_244, pp. 2005-2014.

Shrestha S, Kunwar RM, Jan HA, Abbasi AM, Bussmann RW, Paniagua-Zambrana NY. 2021. Fragaria nubicola Lindl. ex Lac. In: Kunwar RW, Sher H, Bussmann RW. Ethnobotany of the Himalayas. Springer, Cham. doi: 10.1007/978-3030-57408-6_103, pp. 941-950.

Shrestha, I, Kunwar RM, Bussmann RW, Paniagua-Zambrana NY. 2021. Maesa chisia Buch.-Ham. ex D. Don. In: Kunwar RW, Sher H, Bussmann RW. Ethnobotany of the Himalayas. Springer, Cham. doi: 10.1007/978-3-030-574086_146, pp. 1223-1230.

Shyaula SL, Kunwar RM, Bussmann RW, Paniagua-Zambrana NY. 2021. Osyris quadripartida Salzm. ex Decne. In: Kunwar RW, Sher H, Bussmann RW. Ethnobotany of the Himalayas. Springer, Cham. doi: 10.1007/978-3-030-574086_170, pp. 1415-1422.

Siwakoti M, Kunwar RM, Bussmann RW, Paniagua-Zambrana NY. 2021. Ageratina adenophora (Spreng.) R.M. King \& H. Rob. In: Kunwar RW, Sher H, Bussmann RW. Ethnobotany of the Himalayas. Springer, Cham. doi: 10.1007/9783-030-57408-6_14, pp. 171-176.

Subedi CK, Chaudhary RP, Kunwar RM, Bussmann RW, Paniagua-Zambrana NY. 2021. Jatropa curcas L. In: Kunwar RW, Sher H, Bussmann RW. Ethnobotany of the Himalayas. Springer, Cham. doi: 10.1007/978-3-030-57408-6_131, pp. 1111-1122.

Thapa-Magar DK, Thapa-Magar KB, Kunwar RM, Bussmann RW, Paniagua-Zambrana NY, Hussain, W. 2021. Picea smithiana (Wall.) Boiss In: Kunwar RW, Sher H, Bussmann RW. Ethnobotany of the Himalayas. Springer, Cham. doi: 10.1007/978-3-030-57408-6_184, pp. 1507-1514.

Timalsina K, Bhusal A, Kunwar RM, Bussmann RW, Paniagua-Zambrana NY. 2021. Morus alba L., Morus serratua Roxb. In: Kunwar RW, Sher H, Bussmann RW. Ethnobotany of the Himalayas. Springer, Cham. doi: 10.1007/978-3030-57408-6_153, pp. 1295-1308.

Timilsina B, Parajuli R, Rijal DP, Kunwar RM, Jan HA, Hussain W, Bussmann RW, Paniagua-Zambrana NY. 2021. Swertia angustifolia Burkill, Swertia chirayita Buch.-Ham. ex C.B. Clarke, Swertia cordata (G.Don) Clarke, Swertia petiolata D. Don In: Kunwar RW, Sher H, Bussmann RW. Ethnobotany of the Himalayas. Springer, Cham. doi: 10.1007/978-3-030-57408-6_237, pp. 1943-1952.

Tiwari A, Kunwar RM, Bussmann RW, Paniagua-Zambrana NY, Hussain W, Abbasi, AM. 2021. Abies pindrow (Royle ex D. Don.) Royle, Abies spectabilis (D. Don) Spach. In: Kunwar RW, Sher H, Bussmann RW. Ethnobotany of the Himalayas. Springer, Cham. doi: 10.1007/978-3-030-57408-6_6, pp. 107-118.

Uprety Y, Kunwar RM, Bussmann RW, Paniagua-Zambrana NY. 2021. Spondias pinnata (L. f.) Kurz In: Kunwar RW, Sher H, Bussmann RW. Ethnobotany of the Himalayas. Springer, Cham. doi: 10.1007/978-3-030-57408-6_234, pp. 1927-1932. 
Vidaurre PJ, Paniagua-Zambrana NY, Moraes-R M. 2006. Etnobotánica en los Andes de Bolivia. In: Moraes et al. (Eds.) Botánica Económica de los Andes Centrales. pp. 224-238.

\section{BOOKS}

Balslev H, Paniagua-Zambrana NY, Blacutt E, Moraes RM, Macía J, Parada, A, Inturias Y, Perez Z, Teran J, Aliaga .M, Grandez C, Serralta A. 2012. Palmeras de los Leco y sus usos. Herbario Nacional de Bolivia, Universidad Mayor de San Andrés, La Paz.

Beck S, Paniagua-Zambrana NY, López-R P, Nagashiro N (eds). 2010. Memorias del Simposio XXX Aniversario del Instituto de Ecología. LIDEMA-PUMA-UMSA, La Paz, Bolivia. 760 pp.

Beck S, Paniagua-Zambrana NY, Preston D (eds.). 2001. Historia, Ambiente y Sociedad en Tarija, Bolivia. Instituto de Ecología, UMSA/Escuela de Geografía/Universidad de Leeds. Ed. Grafica Latina. La Paz, Bolivia.

Bussmann RW, Paniagua-Zambrana NY (Eds.) 2020. Ethnobotany of Mountain Regions. Springer International Publishing: Cham.

Foster R, Paniagua-Zambrana NY, Wachter T. 1997. Guía Ilustrada de Campo de las Plantas del Parque Nacional y Área Natural de Manejo Integrado Madidi, Depto. La Paz, Bolivia. Field Museum of Natural History of ChicagoConservation International. Chicago, IL.

Paniagua-Zambrana NY, Bussmann RW (Eds.) 2017. La etnobotánica de los Chácobo en el siglo XXI. Herbario Nacional de Bolivia, Instituto de Ecología, Universidad Mayor de San Andrés, William L. Brown Center. Missouri Botanical Garden. (ISBN 978-0-9060231-5-3)

Paniagua-Zambrana NY, Bussmann RW (Eds.) 2020. Ethnobotany of the Andes. Springer International Publishing, Cham. pp. XXXI + 1955, ISBN: 978-3-030-28932-4

Paniagua-Zambrana NY, Bussmann RW, Blacutt E, Macia MJ. (eds.). 2011. Los Chacobo y las Palmeras. Graficart, Trujillo, Peru.

Paniagua-Zambrana NY, Bussmann RW, Blacutt, E, Macía MJ. 2012. Conservando nuestros bosques: conocimiento y uso de las palmeras en las comunidades campesinas del norte de Bolivia. Herbario Nacional de Bolivia, William L. Brown Center, Missouri Botanical Garden. Plural Editores, La Paz, Bolivia.

Paniagua-Zambrana NY, Bussmann RW, Macía MJ. 2012. El bosque SI tiene valor - el uso de palmeras en las comunidades campesinas e indígenas de la región de Inambari, Madre de Dios, Perú. Herbario Nacional de Bolivia, William L. Brown Center, Missouri Botanical Garden. Graficart, Trujillo, Peru.

Paniagua-Zambrana NY, Bussmann RW, Macía MJ. 2012. El conocimiento de nuestros ancestros - los Ese Eja y su uso de palmeras, Madre de Dios, Peru. Herbario Nacional de Bolivia, William L. Brown Center, Missouri Botanical Garden. Graficart, Trujillo, Peru.

Paniagua-Zambrana NY, Bussmann RW, Vega C, Téllez C, Macía M.J. 2012. Nuestro conocimiento y uso de las palmeras - una herencia para nuestros hijos. Comunidades Llaquash, San Martín, Peru. Herbario Nacional de Bolivia, William L. Brown Center, Missouri Botanical Garden. Graficart, Trujillo, Peru (ISBN-13: 978-9848415-3-0).

Paniagua-Zambrana NY, Bussmann RW, Vega C, Téllez C, Macía M.J. 2012. Kampanak se usa para el techo pero ya no hay - Uso y conservación de palmeras entre los Awajun, Amazonas, Perú. Herbario Nacional de Bolivia, William L. Brown Center, Missouri Botanical Garden. Graficart, Trujillo, Peru.

Paniagua-Zambrana NY, Bussmann RW, Vega C, Téllez C, Macía MJ. 2012. Nuestro conocimiento y uso de las palmeras - una herencia para nuestros hijos. Comunidades Llaquash, San Martín, Peru. Herbario Nacional de Bolivia, William L. Brown Center, Missouri Botanical Garden. Graficart, Trujillo, Peru.

Paniagua-Zambrana NY, Bussmann RW, Vega C, Tellez C. 2014. Los Chacobo y su historia en el siglo XX. Herbario Nacional de Bolivia, William L. Brown Center, Missouri Botanical Garden. Herbario Nacional de Bolivia, William L. Brown Center, Missouri Botanical Garden. Graficart, Trujillo, Perú.

Paniagua-Zambrana NY, Moraes-R M, Urioste C. (eds.). 2010. Los Leco usan las palmeras - Guía de usos de palmeras de las comunidades Irimo, Munaypata, Pucasucho, Illipana Yuyo, Santo Domingo y Correo (Apolo, La Paz, Bolivia). Herbario Nacional de Bolivia, Universidad Mayor de San Andrés, La Paz. 
Vega C, Tellez C, Bussmann RW, Paniagua-Zambrana NY. 2014. Yo conozco y cuido las palmeras. William L. Brown Center, Missouri Botanical Garden. William L. Brown Center, Missouri Botanical Garden. Graficart, Trujillo, Perú. 